\title{
Scaling Analysis and Experimental Investigation of a Rotating Detonation Engine
}

\author{
David Thomas Billups \\ West Virginia University, davidtbillups@gmail.com
}

Follow this and additional works at: https://researchrepository.wvu.edu/etd

Part of the Aerodynamics and Fluid Mechanics Commons, Energy Systems Commons, Heat Transfer, Combustion Commons, and the Propulsion and Power Commons

\section{Recommended Citation}

Billups, David Thomas, "Scaling Analysis and Experimental Investigation of a Rotating Detonation Engine" (2019). Graduate Theses, Dissertations, and Problem Reports. 3774.

https://researchrepository.wvu.edu/etd/3774

This Thesis is protected by copyright and/or related rights. It has been brought to you by the The Research Repository @ WVU with permission from the rights-holder(s). You are free to use this Thesis in any way that is permitted by the copyright and related rights legislation that applies to your use. For other uses you must obtain permission from the rights-holder(s) directly, unless additional rights are indicated by a Creative Commons license in the record and/ or on the work itself. This Thesis has been accepted for inclusion in WVU Graduate Theses, Dissertations, and Problem Reports collection by an authorized administrator of The Research Repository @ WVU. For more information, please contact researchrepository@mail.wvu.edu. 


\title{
Scaling Analysis and Experimental Investigation of a Rotating Detonation Engine
}

\author{
David T. Billups \\ Thesis submitted \\ to the Benjamin M. Statler \\ College of Engineering and Mineral Resources \\ at West Virginia University \\ in partial fulfillment of the requirements for the degree of \\ Master of Science in \\ Aerospace Engineering \\ Andrew C. Nix, Ph.D., Chair \\ Donald H. Ferguson, Ph.D. \\ Patrick H. Browning, Ph.D. \\ Department of Mechanical and Aerospace Engineering \\ Morgantown, West Virginia \\ 2019
}

Keywords: Dimensional analysis, Detonation, Rotating detonation engine, Pressure gain combustion, Dynamic pressure measurement

Copyright 2019 David T Billups 


\title{
Abstract \\ Scaling Analysis and Experimental Investigation of a Rotating Detonation Engine
}

\begin{abstract}
David T. Billups
Pressure gain combustion (PGC) technologies, specifically rotating detonation engines (RDEs), are poised to provide the next big leap in gas turbine engine advancement, significantly increasing the thermal. RDEs make use of thermodynamic advantages of isochoric as opposed to isobaric combustion. Theorized to increase thermal efficiency by up to 7\% [1], the RDE would have significant impact on reducing anthropogenic carbon emissions. In addition to efficiency gains, the RDE also provides mechanical simplicity and reduced size advantages compared to it's traditional counterparts and PGC competition.

The United States (U.S.) Department of Energy (DOE) National Energy Technology Laboratory (NETL) maintains and operates two rotating detonation combustor (RDC) facilities. Firstly, a 6 inch diameter lab scale RDE (LSRDE) is utilized to better understand the operational regime. Secondly, the bench scale RDE (BSRDE) enables optical access within the plenums to investigate the dynamic interactions at the injection inlet.

This work begins to investigate the relationships between the NETL facilities. By performing a dimensional analysis on the RDC system and creating a data reduction routine to more similarly compare data from the two facilities, it was found that there is little connection between the two experimental rigs. It is believed that the primary cause of this disconnection is the significant difference in physical mechanisms driving the shock and detonation waves in each respective facility. However, the methodology presented in this work does begin to reveal the interaction of system parameters and could prove to be useful as RDE operation becomes better understood.
\end{abstract}




\section{Dedication}

Be glad of life because it gives you the chance to love, to work, to play, and to look up at the stars.

$\sim$ Henry Van Dyke

Remember to look up at the stars and not down at your feet. Try to make sense of what you see and wonder about what makes the universe exist. Be curious. And however difficult life may seem, there is always something you can do and succeed at. It matters that you don't just give up.

Stephen Hawking, R.I.P.

I would like to dedicate this work to my friends and family. Of particular mention are my partner, Ali; my father, John; my mother, Kathy; my grandfather, Fred; and a friend who needs no introduction, Maggie. Firstly, thank you for your lessons of strength, leadership, and laughter. They have served me well throughout graduate school. Without your enduring support, patience, and love, this journey would surely be more difficult. Thank you for your forgiveness in missing out on some undoubtedly good memories. Here's to many more. A final special thanks to Ali, for your understanding, your support, your loyalty, and your love. 


\section{Acknowledgements}

If you aren't in over your head, how do you know how tall you are?

$\sim$ T. S. Eliot

Make the most of yourself, for that is all there is of you.

$\sim$ Ralph Waldo Emerson

I would like to begin by expressing gratitude toward Dr. Andrew Nix. I would be without many significant life experiences without your guidance and mentorship. After six (yes, six!) years of working together in your lab, on the EcoCAR3 project, and in graduate school, I think of you as a close friend, and for that I am grateful. We have accomplished quite a lot, but there's always time for fishing stories.

Many thanks go out to the remainder of my committee. Thank you, Dr. Don Ferguson, for your mentorship and patience as I learned the ways of NETL and graduate school. Additional appreciation goes to Dr. Ferguson for his thoughtful answer to my questions, no matter how relevant they were. Thank you to Dr. Patrick (Pat) Browning for your support and reality checks throughout my career at WVU. It is amazing how comforting it can be to find like-mindedness in colleagues and mentors in your field. I am so fortunate to have found that in each of you. Our talks about whitewater, the Cranberry Glades, mountaineering, and engineering helped to convince me that pursuing this degree was the right choice. An additional thank you to each of my committee members for agreeing to sit on my graduate committee.

I owe a big thank you to my colleagues at NETL who helped me along the way; Pete Strakey, Todd Sidwell, Geo Richards, Doug Straub, Arnab Roy, Clinton Bedick, Pankaj Saha, Bridget O’Meara, Ethan Knight, Kristyn Johnson, Joe Yip, Mark Tucker, and Jeff Riley. Special thanks to Jeff Riley for helping me keep my head up and always greeting me with a smile. I am so fortunate and thankful for the opportunity at NETL. The ORISE program has proven to be a tremendous learning experience. Thank you to the ORISE staff and organizers.

Lastly, I would like to thank my friends and family once again. No more worrying or wondering we've done it! Thank you for your continued interest in my life and patience when I struggle to share it. 


\section{Table of Contents}

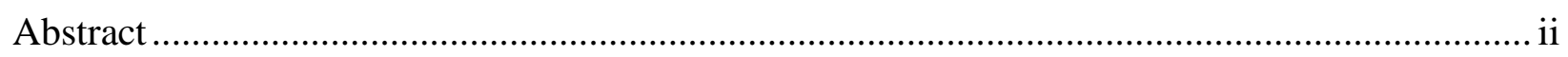

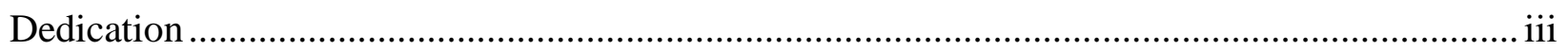

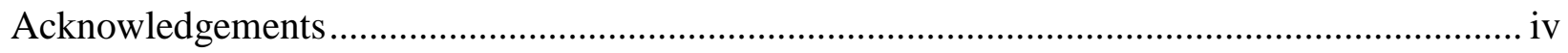

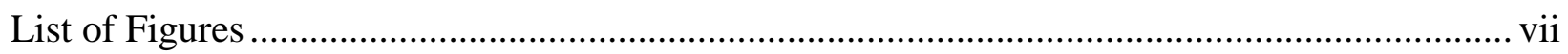

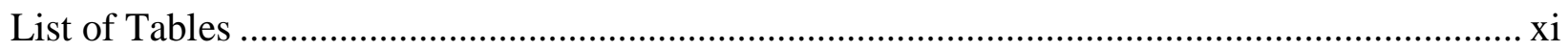

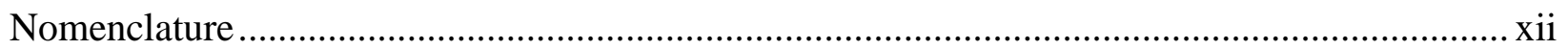

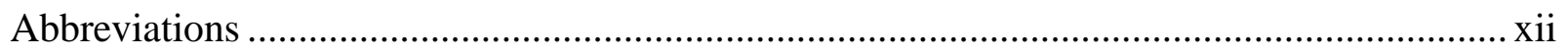

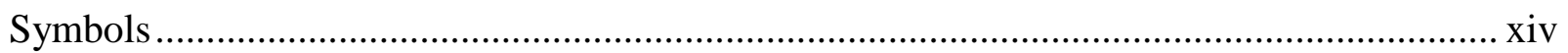

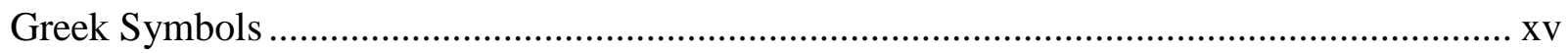

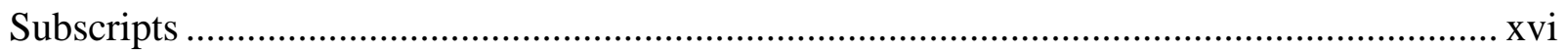

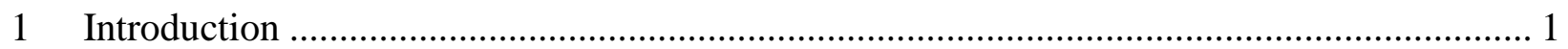

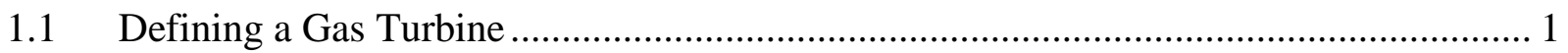

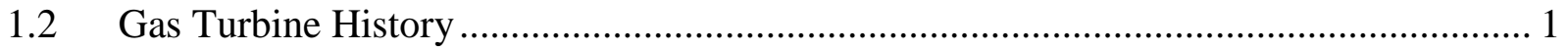

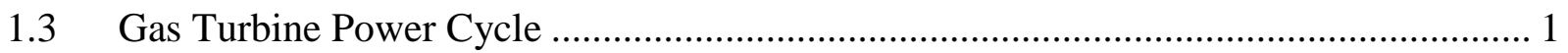

1.4 Detonation versus Deflagration Combustion ……………………............................. 3

1.5 Detonation combustion history: .......................................................................... 4

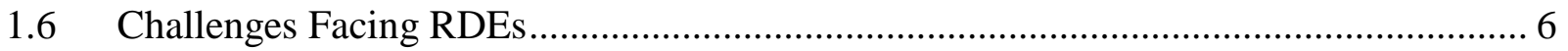

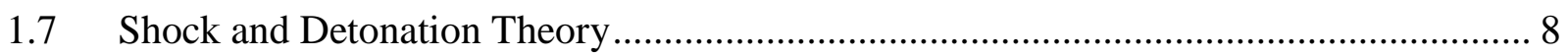

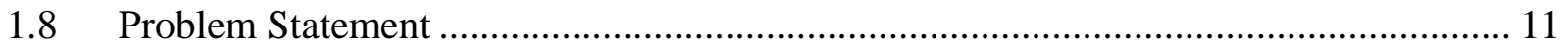

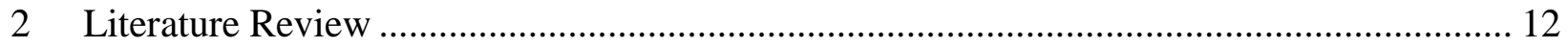

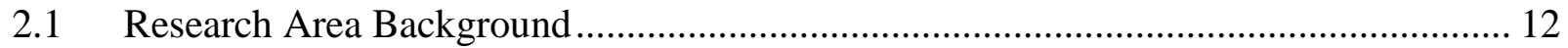

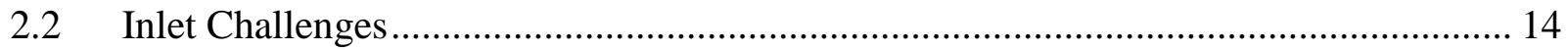

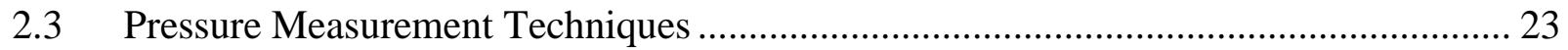

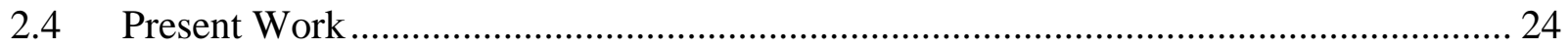

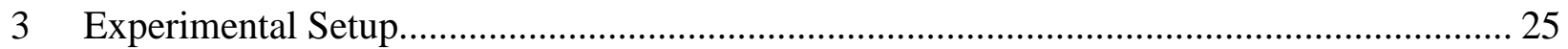

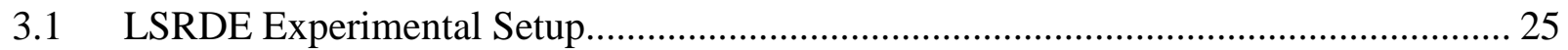

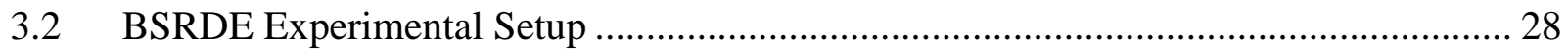

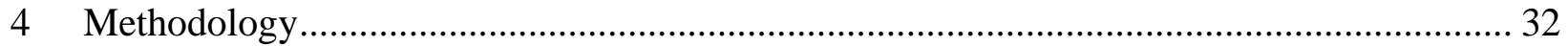

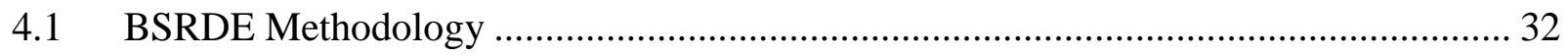

4.1.1 Dimensional Analysis and Buckingham Pi Theorem ……………………........... 32

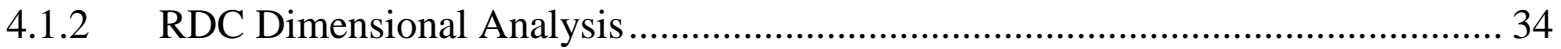

4.1.3 BSRDE Detonation Characteristics Determination .............................................. 38

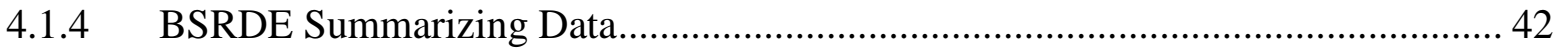




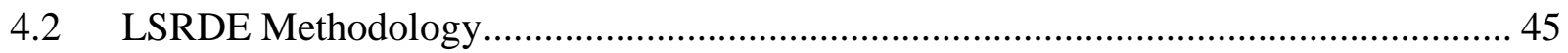

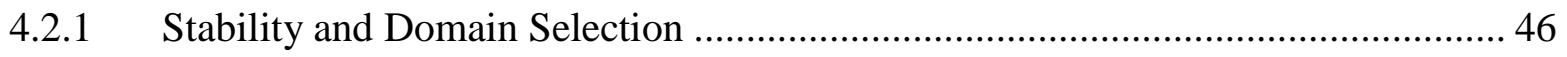

4.2.2 LSRDE Detonation Characteristics Determination .............................................. 51

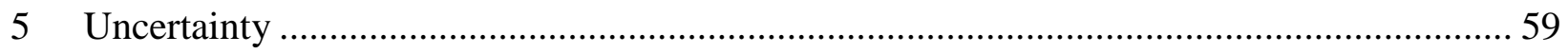

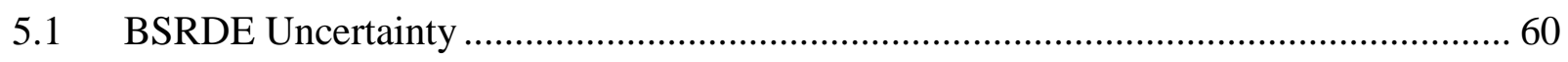

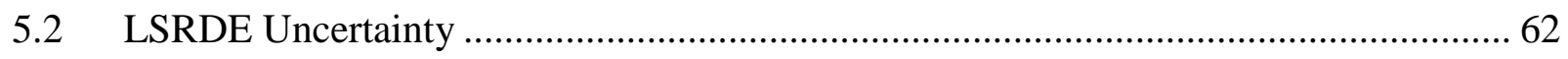

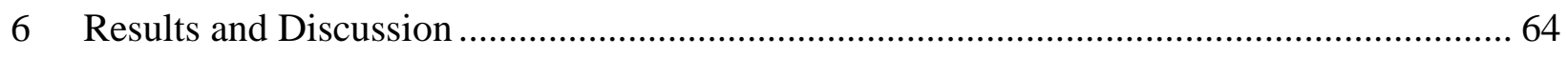

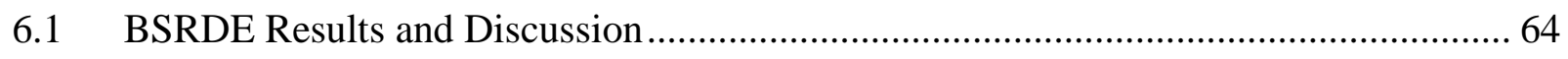

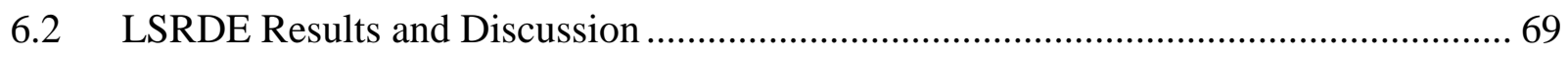

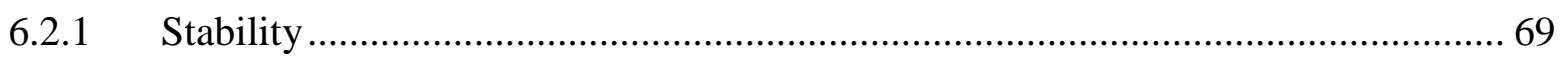

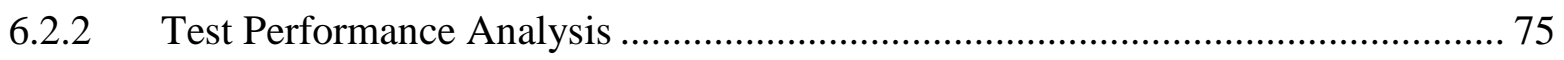

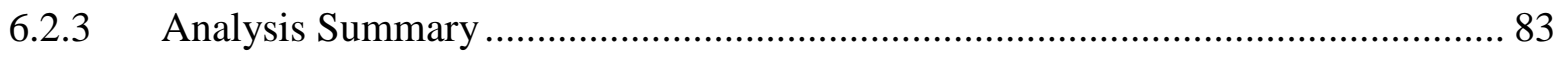

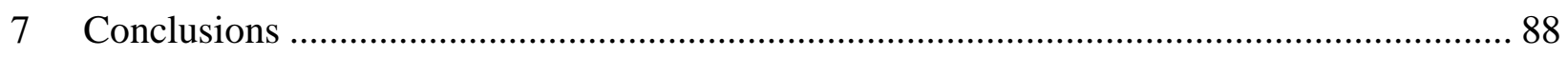

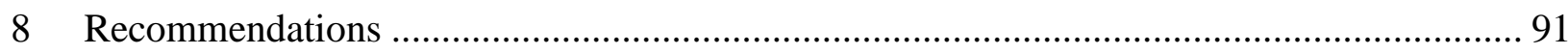

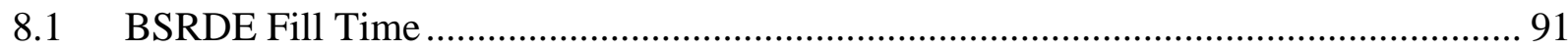

8.2 LSRDE Measurement Techniques ..................................................................... 93

8.2.1 Cooled Dynamic Pressure Transducers ................................................................ 93

8.2.2 Wave Speed via Image Processing ………........................................................ 93

8.3 LSRDE Configuration ...................................................................................... 94

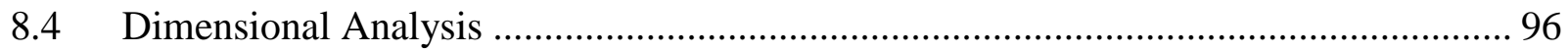

8.5 Characterization of LSRDE Detonation Strength ...................................................... 96

8.6 Multi-wave Consideration in Data Reduction.............................................................. 97

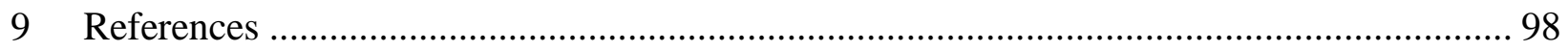

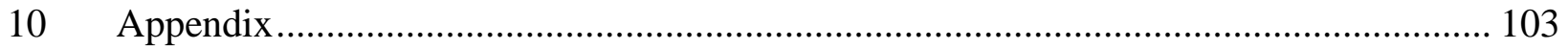

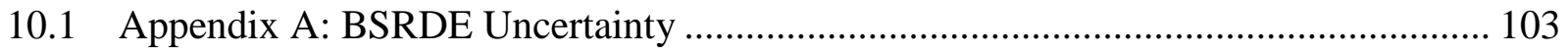

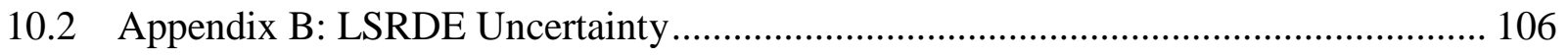

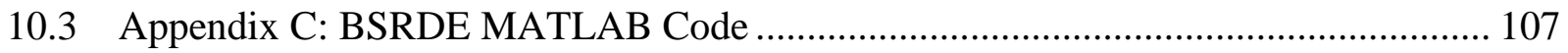

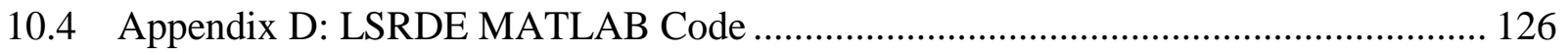

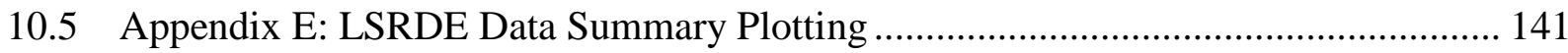

10.6 Appendix E: FWHM MATLAB Function .......................................................... 156

10.7 Appendix F: Detonation Characteristics Determination MATLAB Function .............. 159 


\section{List of Figures}

Figure 1.1: Common power cycles (Edited from [6]) ................................................... 2

Figure 1.2: Detonation Pressure Profile under ZND Theory [20] ........................................ 9

Figure 1.3: P-v Diagram for a representative detonative process [22] ................................. 10

Figure 2.1: Visual Representation of Interruption Time.................................................. 14

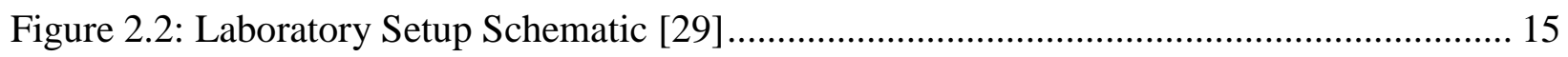

Figure 2.3: Plot of dimensionless terms for changing (a) geometry and (b) fuel type [29]......... 16

Figure 2.4: x-t diagrams depicting shock reflections within cavity [29] ............................... 16

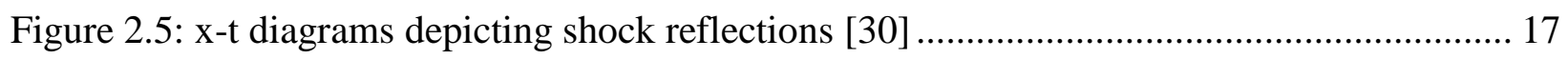

Figure 2.6: Signal Response for different measurement locations and types at mass flows of (left)

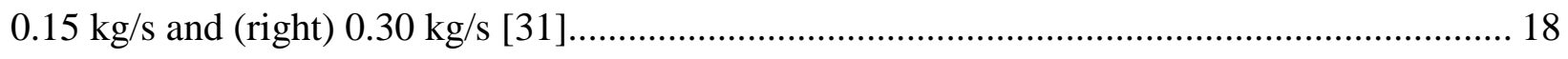

Figure 2.7: Operational Map and Indicators for LPD Condition [16] .................................... 20

Figure 2.8: FFT and Spectrogram Comparison at $\phi=1.0$ and air supply flow rates of (top) $0.2 \mathrm{~kg} / \mathrm{s}$,

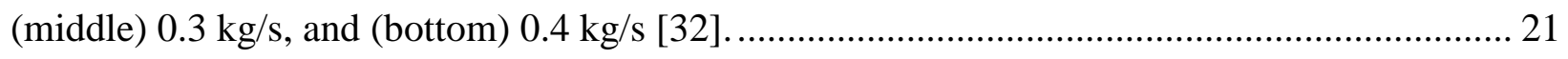

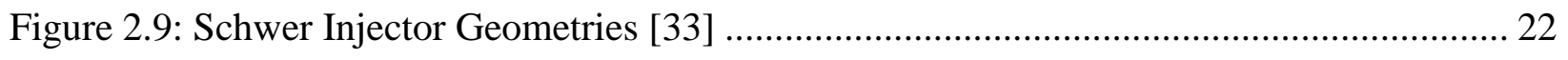

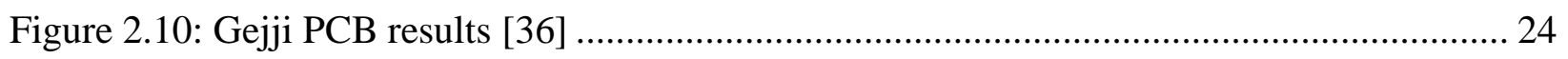

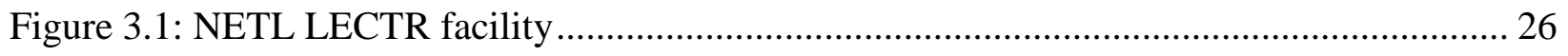

Figure 3.2: LSRDE Cross Sectional Geometry …....................................................... 27

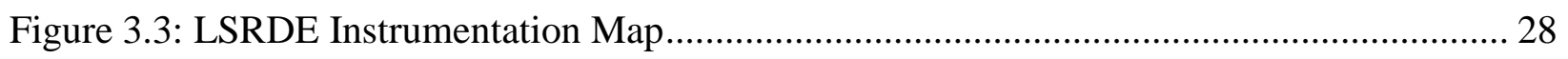

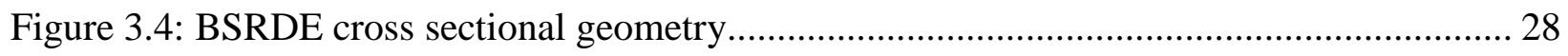

Figure 3.5: Aluminum housing and 3D printed reference inlet ........................................ 29

Figure 3.6: BSRDE Reference Test Article .............................................................. 30

Figure 3.7: Picture of BSRDE facility instrumentation .................................................. 30

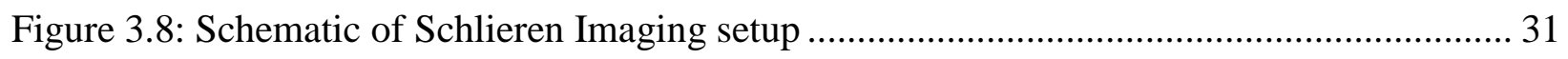


Figure 3.9: BSRDE Instrumentation Schematic

Figure 4.1: BSRDE Geometry and Setup ...................................................................... 34

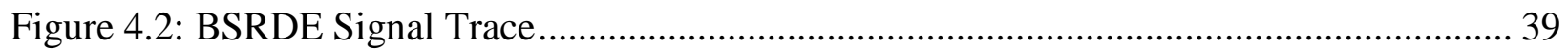

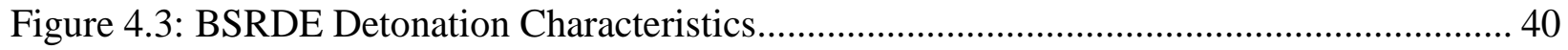

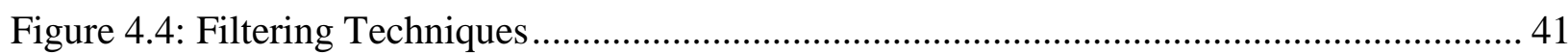

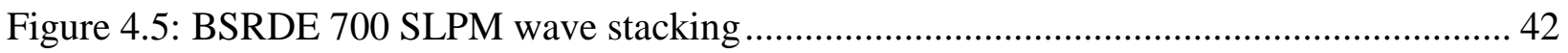

Figure 4.6: Effect of Air Supply Flow Rate on Pressure ratio and interruption time ................. 43

Figure 4.7: Variation in the parameters which make up the $\Pi_{2}$ term ...................................... 44

Figure 4.8: $\Pi_{2}$ versus interruption time ratio for varying air supply flow rates ..................... 45

Figure 4.9: Raw signal for a representative case .............................................................. 46

Figure 4.10: Effect of Butterworth filtering for temperature drift ..................................... 47

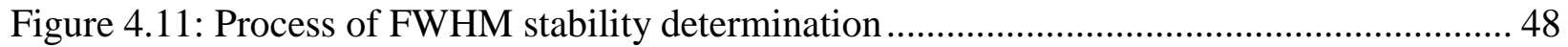

Figure 4.12: Full-Width, Half Maxima at varying time windows .......................................... 49

Figure 4.13: Spectrogram and FWHM for a representative case ......................................... 50

Figure 4.14: Detonation characteristics for a representative case......................................... 51

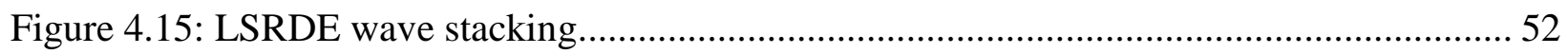

Figure 4.16: Probability distribution of peak pressures ....................................................... 53

Figure 4.17: Distribution of pressure peaks and interruption time ratio................................. 54

Figure 4.18: $\Pi_{2}$ versus interruption time ratio for a representative case ................................ 54

Figure 4.19: Interruption time versus time, showing discretized results due to low sample rate. 55

Figure 4.20: Contour plot of dimensionless parameters …................................................ 56

Figure 4.21: Average wave shape plotted with minimum and maximum deviations................ 58

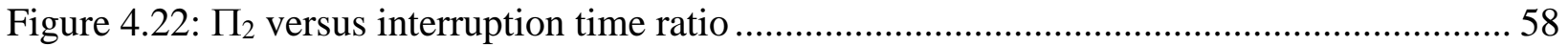


Figure 6.1: BSRDE Wave Stacking 65

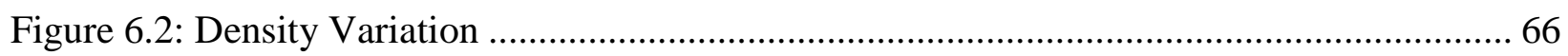

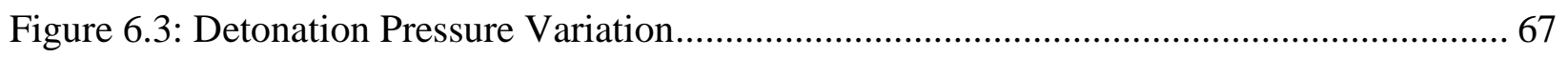

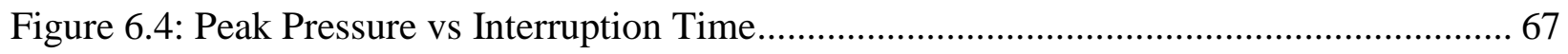

Figure 6.5: Dimensionless Pressure, $\Pi_{2}$ versus Interruption Ratio …………………………..... 68

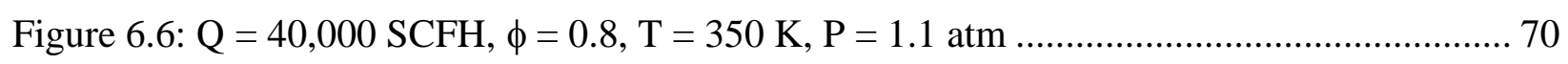

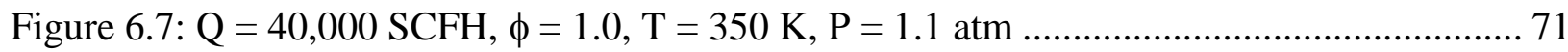

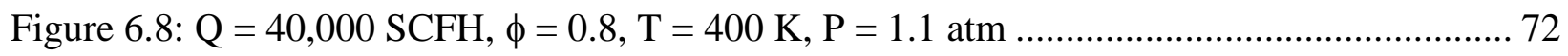

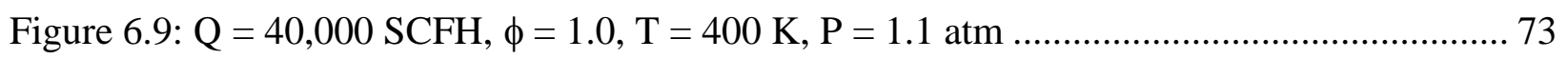

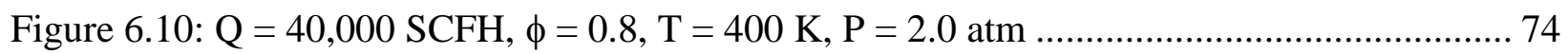

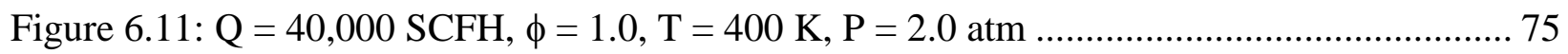

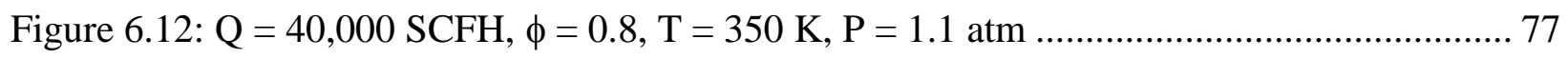

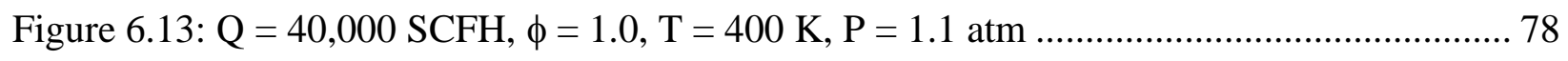

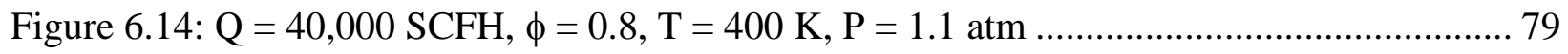

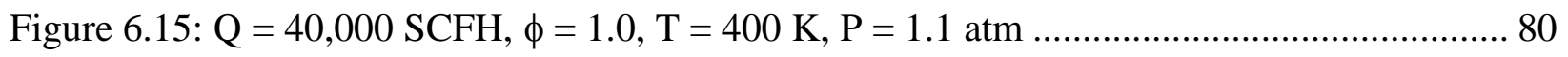

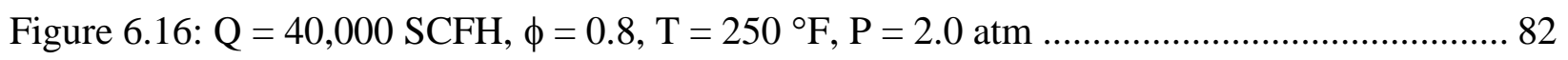

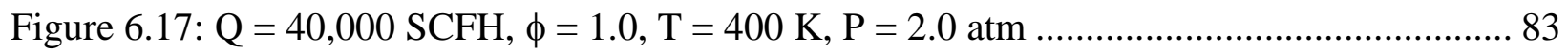

Figure 6.18: Average Wave Forms plotted together by test conditions ....................................... 84

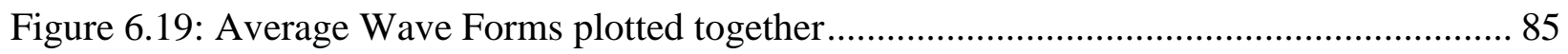

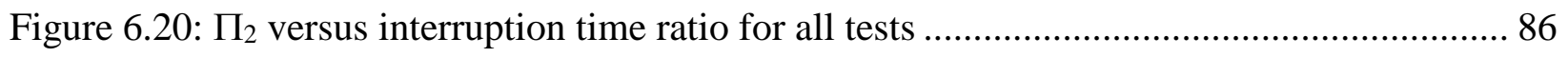

Figure 6.21: Averaged $\Pi_{2}$ versus interruption time ratio for all tests with uncertainty................ 87

Figure 8.1: BSRDE Pre-detonation Tube Schematic................................................................ 92 
Figure 8.2: Radially averaged luminosity within the RDC annulus plotted over annulus angular extent and run time, together with a measured sample characteristic path line (a), showing stable one-wave operation. Detonation wave speed for a subset of laps, as calculated based on the luminosity characteristics, together with their moving average and the short-time FFT of PCB 06 (b). A good agreement of pressure and luminosity measurement results confirms the ability to also reveal lap-to-lap fluctuations in wave speed that are masked by the FFT. [41] ....................... 94 Figure 8.3: Images of Experimental Rig after run, supporting argument for greater mixing at air

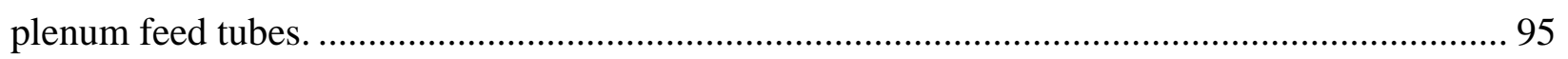
Figure 8.4: LSRDE Facility at NETL, depicting air plenum supply plumbing ........................ 96 


\section{List of Tables}

Table 3.1: LSRDE Instrumentation and Data Acquisition ................................................... 27

Table 4.1: Common Dimensionless Terms............................................................................ 33

Table 4.2: BSRDE data matrix (Number of Data Sets for Each Set of Test Conditions) ............ 45

Table 4.3: Testing Matrix (Number of cases at each set of conditions). All cases at air supply flow

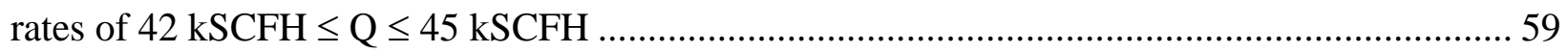

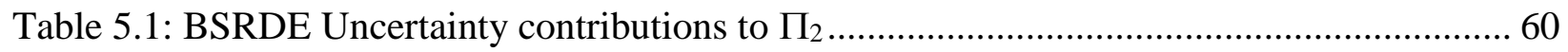

Table 5.2: BSRDE Uncertainty for Various Air Supply Flow Rates .................................... 61

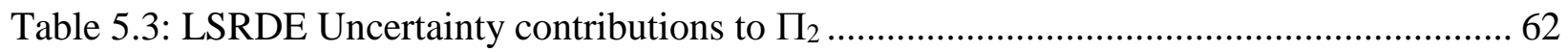

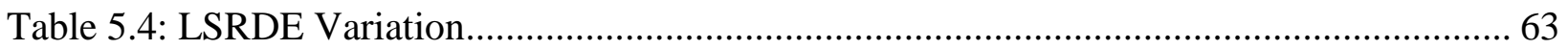




\section{Nomenclature}

\section{Abbreviations}

3D

AEO

AFRL

BC

BSRDE

CFD

CI

CJ

CTAP

DDT

DOE

EIA

EPA

FAR

FFT

FLT

FWHM

$\mathrm{Hz}$

ITP

$\mathrm{kHz}$

LCJ

LPD
Three-Dimensional

Annual Energy Outlook

Air Force Research Laboratory

Before Christ

Bench Scale RDE

Computational Fluid Dynamics

Confidence Interval

Chapman-Jouget

Continuous Tube Attenuated Pressure

Deflagration to Detonation Transition

Department of Energy

Environmental Information Administration

Environmental Protection Agency

Fuel to Air Ratio

Fast Fourier Transform

Force, Length, Time

Full Width, Half Maxima

Hertz

Infinite Tube Pressure

Kilohertz

Lower Chapman Jouget

Longitudinal Pulsed Detonation 


\begin{tabular}{ll} 
LSRDE & Lab Scale RDE \\
MHz & Megahertz \\
MLT & Mass, Length, Time \\
MPa & Megapascal \\
NETL & National Energy Technology Laboratory \\
NOx & Nitrogen Oxide \\
PCB & PicoCoulomB Piezotronics Corp \\
PDE & Pulse Detonation Engine \\
PDF & Probability Density Function \\
PSD & Power Spectral Density \\
PT & Pressure Transducer \\
RDC & Rotating Detonation Combustion \\
RDE & Rotating Detonation Engine \\
SCFH & Standard Cubic Feet per Hour \\
SLPM & Standard Liters Per Minute \\
TC & Thermocouple \\
UC & University of Cincinnati \\
UCJ & Upper Chapman Jouget \\
U.S. & United States \\
ZND & Zeldovich-von Neumann-Doring \\
\hline
\end{tabular}




\section{Symbols}

A

B

C

$\mathrm{Ca}$

D

E

$\mathrm{Eu}$

$E_{V}$

F

$\mathrm{f}$

fill

fire

$\mathrm{h}$

K

$\mathrm{k}$

1

$\mathrm{L}$

M

$\mathrm{m}$

$\mathrm{N}$

n

$\mathrm{p}$
Area, Initial Thermodynamic State

Final Thermodynamic State for Isochoric Process

Final Thermodynamic State for Isobaric Process

Cauchy Number

Upper Chapman-Jouget Point

Lower Chapman-Jouget Point

Euler Number

Bulk Modulus

Force

Function of; frequency

Pre-detonator fill sequence

Ignition indication

Height

Area Ratio

Number of variables in dimensional analysis

Length

Length

Mach Number, Mass

Mass

Number of fuel injection holes; Number of samples

Number of Pi Terms

Pressure 


\begin{tabular}{|c|c|}
\hline $\mathrm{P}$ & Pressure, Probability \\
\hline Q & Volume Flow Rate \\
\hline & Independent variables in dimensional analysis, \\
\hline 1 & Heat addition per unit mass \\
\hline $\mathrm{r}$ & Radius; Number of dimensions in dimensional analysis \\
\hline $\operatorname{Re}$ & Reynolds Number \\
\hline S & von Neumann Spike \\
\hline $\mathrm{St}$ & Strouhal Number \\
\hline $\mathrm{T}$ & Temperature, Time \\
\hline Tube & Pre-detonator Tube Characteristic \\
\hline $\mathrm{t}$ & Time, Student-t Variable \\
\hline $\mathrm{U}$ & Velocity \\
\hline $\mathrm{W}$ & Width \\
\hline $\mathrm{x}$ & Length \\
\hline $\mathrm{Z}$ & Z-Score (in Normal and Student-t Distribution) \\
\hline \multicolumn{2}{|c|}{ Greek Symbols } \\
\hline$\mu$ & Kinematic Viscosity \\
\hline$v$ & Specific Volume \\
\hline$\Pi$ & Dimensionless Variable \\
\hline$\rho$ & Density \\
\hline$\tau$ & Period \\
\hline$\phi$ & Equivalence Ratio \\
\hline$\omega$ & Rotational Frequency \\
\hline
\end{tabular}




\section{Subscripts}

0,1

$\infty$

a

c

ch

CJ

det

f

g

i

INT

o

$\mathrm{p}$

$\mathrm{p}$

PD

stoich
Initial

Infinity

Air

Chord

Channel

Chapman-Jouget

Detonation

Fuel

Air gap

Inner

Interruption

Outer

Peak

Plenum

Pre-Detonator Tube

Stoichiometric 


\section{Introduction}

\subsection{Defining a Gas Turbine}

Gas turbines have many applications. Primarily, these complex machines are used in land-based power generation and propulsion of commercial aircraft as well as large ships, though they are also found in pipeline compressor stations. Turbines are desired for their ability to perform efficiently at a constant operating condition. This makes them ideal for fossil fuel burning power plants and cruise applications. In comparison, the more traditional internal combustion engine of automobiles operates at around 30\% efficiency, while gas turbine combined cycle power plants have recently surpassed $60 \%$ operating efficiency.

\subsection{Gas Turbine History}

The invention of the gas turbine is credited to John Barber, who's 1791 patent outlined the key components found in modern gas turbines; namely, a compressor, combustor, and turbine [2]. Initial developments of gas turbines began prior to World War II, with the goal of electrification. Due to the war, innovation was driven by the military as turbines demonstrated an ability to deliver a step change in speed. In 1937, the first turbojet was tested in England, designed by Frank Whittle. Just two years later, the first turbojet powered plane completed test flights in Germany when a Heinkel HE 178 was outfitted with turbojets designed by Hans Pabst von Ohain. Fascinatingly, these two inventors filed patents independently, with no knowledge of the other's work [2-4]. By the 1970s, modern gas turbines had come to form, and the high bypass ratio turbofan made way for the wide-body commercial aircraft seen today.

\subsection{Gas Turbine Power Cycle}

In order to produce work in a turbine, a pressure ratio must be provided. This pressure ratio is provided to the working fluid by the compressor upstream of the turbine. In an ideal case with no losses, a working fluid could be compressed, then expanded through the turbine such that the work 
produced by the turbine would exactly drive the compressor. To successfully generate a net-work output, energy can be added to the working fluid via heat addition; in this case, combustion. The figure below shows idealized pressure versus specific volume $(P-v)$ and temperature versus entropy (T-s) diagrams for four of the most common power generation cycles. The Otto Cycle is used by automobile engines. The Diesel cycle is used in diesel engines for automobiles, trains, and ships. Gas turbines make use of the Brayton Cycle, which in the ideal case involves isentropic compression, isobaric (constant pressure) combustion, isentropic expansion, and isobaric heat rejection. In reality, there is a slight pressure loss through the combustor, which is typically between 2 and 8 percent of the compressor exit static pressure [5].
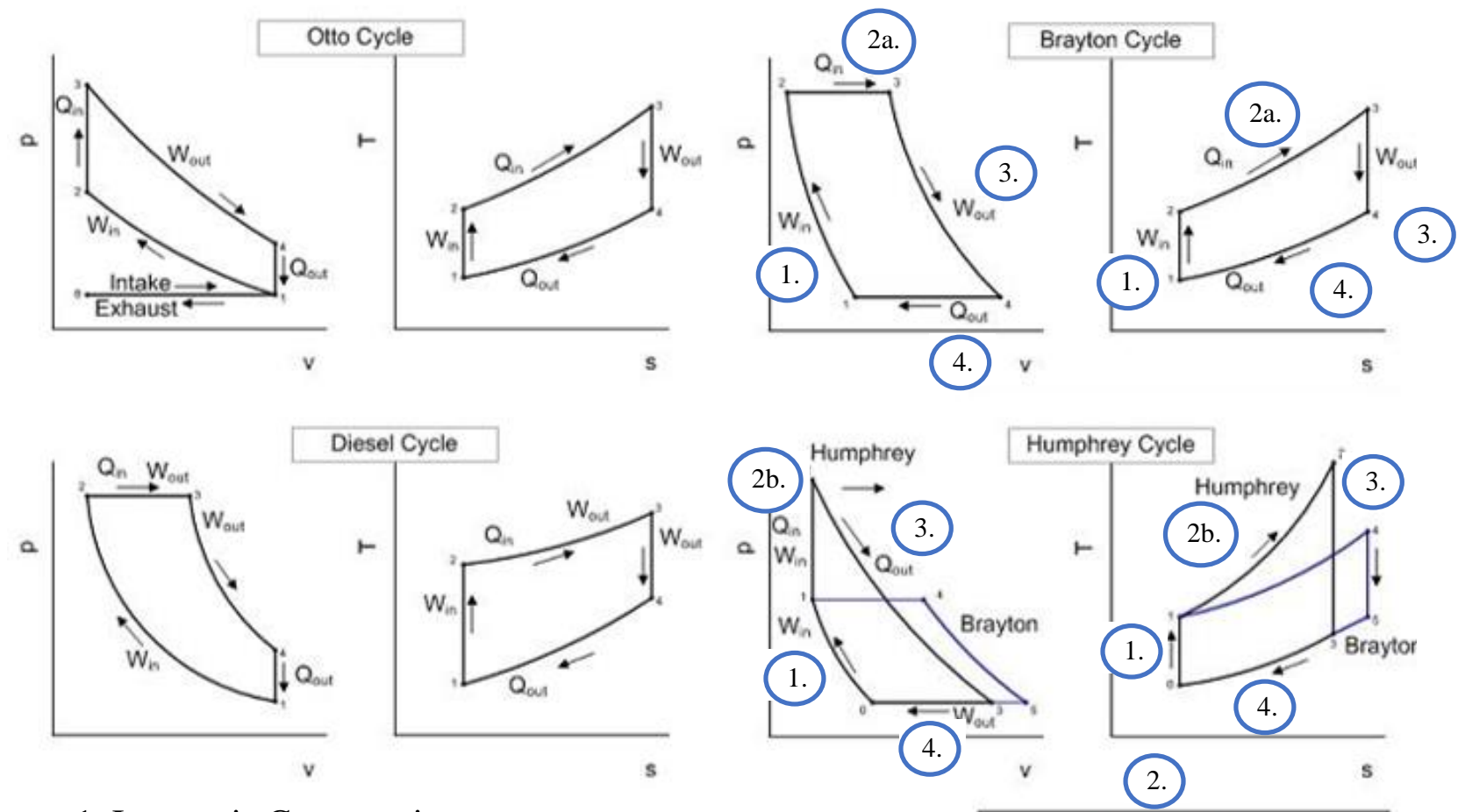

1. Isentropic Compression

2a. Isobaric Combustion

3. Isentropic Expansion

4. Isobaric Heat Rejection

2b. Isochoric Combustion

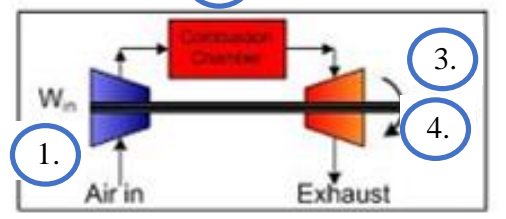

Figure 1.1: Common power cycles (Edited from [6]) 


\subsection{Detonation versus Deflagration Combustion}

The last of the power cycles portrayed in Figure 1.1, the Humphrey Cycle, is used in pressure gain combustion systems, including rotating detonation engines (RDEs). The difference between the ideal Brayton and Humphrey cycles is the mechanism for adding heat to the system. In the Brayton Cycle, deflagration, or subsonic combustion is used, thus the diagrams depict isobaric (constant pressure) combustion. In the Humphrey Cycle, however, detonation is used and therefore isochoric (constant volume) combustion is shown. The Humphrey Cycle is used to describe both pulsed detonation engines (PDEs) and rotating detonation engines. Neither PDEs nor RDEs undergo perfectly isochoric combustion reactions, however it can be approximated to be an isochoric heat addition process because detonation combustion occurs nearly instantaneously through a local volume of reactants. Through these diagrams, the benefits of RDEs is begun to be realized.

As mentioned previously in defining the differences between deflagration and detonation, RDEs have a higher pressure availability within the working fluid, creating more potential to do work. This greater pressure availability is due to the pressure gain generated by the detonation event. Nordeen [1] finds that an engine operating on the Humphrey cycle could perform at a 7-8\% greater thermal efficiency when compared to a Brayton engine operating at similar isentropic compression ratios. In general, the constant volume, detonation combustion event generates less entropy and higher flow work availability, allowing the RDE to operate at these higher thermal efficiencies. As a result, RDEs require less fuel consumption, and conversely, create a greater power density.

In general, pressure gain combustion efforts are motivated by increased efficiency for gas turbine engine applications. Historically, the gas turbine engine increases $0.5 \%$ in efficiency each year[7]. These efficiency increases typically result from an increased compression ratio or increased turbine inlet temperature. Compressors are extremely expensive and complex pieces of machinery, 
making the compression ratio a challenging parameter to manipulate. Temperature increases are facilitated by materials development and new cooling strategies. In the hot section of a gas turbine engine, temperatures can exceed the material limits. For the components to survive, complex cooling strategies redirect relatively cooler air from the compressor exit to components in direct exposure to the hot gas mixture. Material coatings can also be employed to increase the melting point of the surface exposed to the hot gas path. These materials include ceramics, however variation in thermal expansion properties cause significant challenge in thermal barrier coating techniques. Turbine cooling specifically focuses on internal and film cooling. Internal cooling allows for convective cooling through small channels machined into the components. Film cooling permits the coolant flow to seep out of the components into the hot gas path to insulate the surface of the component from the hot gas. An example film cooling technique is discussed in the work of Hayes [8].

The 7-8\% jump mentioned previously would offer drastic improvement and significant reduction in the production of anthropogenic green house gases. The latter detail is significant due to the Environmental Protection Agency's (EPA) and Environmental Information Administration's (EIA) estimates and projections for energy consumption $[9,10]$. The EPA finds that in $201728 \%$ of U.S. generated anthropogenic greenhouse gas emissions were attributed to electricity. Furthermore, the EIA Annual Energy Outlook 2019 report projects that electricity generation from natural gas will increase by $35 \%$ by 2050 . Combined, these facts reveal the urgency for pressure gain technologies.

\subsection{Detonation combustion history:}

Zeldovich, von Neumann, and Doring are independently credited with the first efforts in detonative combustion research. Zeldovich is known for his work in the development of pulse detonation 
engines (PDEs) in the 1950s [11]. These concepts were furthered by Nichols, et al. [12] by attempting to develop an experimental engine which makes use of continuous detonation processes. While the RDE was theorized several decades ago, the instrumentation necessary to temporally resolve RDE system would not be until much later. Today, with piezoelectric and piezoresistive pressure transducers, the propagation of detonation waves can be accurately temporally measured. As a young field, there is plenty of work to be done in optimizing these systems. Since their theoretical origins, detonative combustion has been explored in two primary engine applications, collectively referred to as pressure gain combustion devices.

First, pulse detonation engines (PDE) were developed and investigated, motivated by the thermodynamic advantages of creating a device which made use of constant volume combustion. A PDE fills a cylindrical combustion chamber with a fuel and air mixture. The tube is then sealed off from the supply mixture. A combustion reaction is initiated which coalesces and propagates into a detonation wave. The combusted products then expand out of a nozzle at the open downstream end, yielding thrust. In order to produce relatively constant performance, these devices cycle quite fast, typically between 100-500 Hz. Due to their fill, fire, purge cycles, the exit flow can be considerably periodic, and therefore not ideal for traditional turbine operation. Additionally, to reduce exhaust flow periodicity by increasing cycling frequency, valving mechanisms were challenged. Lobe shaped reed valves and flow type ball valves were utilized to operate PDEs, however, neither provided the robustness or reliability necessary for proposed applications. Each valve technique suffered from high cycle fatigue and thermal stressing.

Second, Rotating Detonation Engines (RDEs) gained researchers' interest as it was theorized that the combustor exit flow would be much less periodic. The first operable RDE is credited to Voitsekhovskii, who designed, built, and tested a vacuum-driven, premixed RDE using an 
acetylene-air mixture [13]. Now, RDEs are desired for commercial aircraft engines, rocket propulsion, power generation, and many more applications.

Mechanical simplicity, lack of valving requirements, and near-steady exhaust conditions therefore, are advantages of RDEs compared to PDEs.

\subsection{Challenges Facing RDEs}

Many challenges exist for the successful operation and implementation of an RDE. These include backflow, mode switching, wave bifurcation, mixing, emissions reduction, and turbine integration. Though each of these require separate consideration and investigation, all are intertwined as will be discussed in the following paragraphs.

One major issue RDE researchers are facing is the backflow of combustion products through the inlet. Due to the pressure gain provided by the detonation event, the combusted products are pushed upstream. This adverse pressure gradient also allows shock waves to propagate into the fuel and air supply plenums. Backflow interrupts the flow of fresh reactants into the combustion chamber, potentially resulting in shock dissipation, wave bifurcation due to changing local equivalence ratios, and other potentially detrimental issues. In a numerical study intended to better understand stable operating modes of an RDE and how those modes would integrate into a modern turbine arrangement, Frolov, et al. found that the fluctuation in the mean static pressure at the inlet was $10 \%$ higher than those fluctuations at the exhaust plane. This suggests the significance of the backflow problem and the need for a robust inlet design [14].

Rotating detonation engines can observe a variety of operational modes and conditions. There are stable and unstable modes, each of which have subsidiaries. Co-rotating single or multi-wave operation are examples of stable modes. Transverse operation is an example of an unstable mode. Stable modes are differentiated from unstable modes by analyzing the periodicity of the system 
and the completeness of the combustion reaction. Generally, the LSRDE at NETL operates in a 2wave, stable mode [15].

Wave bifurcation can occur throughout RDE operation. Bifurcation can be triggered by several factors, resulting in an increased number of waves. At NETL, the LSRDE has been found to be sensitive to wave bifurcation due to increased air supply pre-heat and increased back-pressure. Increased pre-heat works to accelerate the chemical reactions, which allows for auto-ignitions to build into separate detonations. It is also seen that at later times during the experiment, mode switching is more likely to occur. This is theorized to be a result of component heating which changes the reactivity of the reactant mixture. RDE mode can also be influenced by localized differences in equivalence ratio, which have been observed to induce bifurcation [16].

Mixing challenges prove to have widespread implications throughout the RDE. Driscoll, et al. find through a numerical study that by increasing fuel mass flux, decreasing fuel injection area, locating the fuel injectors upstream of the annulus into the air injection slot, and increasing back pressure can have the effect of increasing mixedness [17].

Injection schemes are classified as either premixed or non-premixed. The Rotating Detonation Combustor (RDC) at NETL operates on non-premixed injection [15]. Disagreement with premixed versus non-pre-mixed largely revolve around the concern of flashback in a pre-mixed combustor, a phenomenon during which the upstream reactants are ignited, threatening the integrity of upstream components [18].

Hariharan, et al. showed through a computational study comparing various injection geometries, that the mixing efficiency in a nozzle with perpendicularly oriented fuel injection can be improved by manipulating the injection geometry while maintaining comparable pressure losses [19]. 
The exhaust conditions have several challenges associated with them. One is emissions reduction, which requires the optimization of other aspects of RDE operation that influence the behavior of the combustion reaction and therefore influence the generation of undesirable emissions. For example, mixedness can effect the proportion of reactants that are combusted via deflagration and detonation, which impacts the products that are generated. Depending on conditions, undesirable emissions such as $\mathrm{NO}_{\mathrm{x}}$ can be prominent. Turbine integration is also a challenge associated with exhaust conditions. This is due to the unsteadiness of the exhaust flow as a result of the periodic nature of the system.

\subsection{Shock and Detonation Theory}

Shock waves are familiar by name, however are complex and sensitive phenomena. Physically, they occur in many engineering applications and can occur when local fluid velocity approaches, meets, or exceeds the local speed of sound. These three scenarios are known as transonic, sonic, and supersonic. It is important to note the significance of local conditions in the determination of the speed of sound. In a steady, isentropic, and calorically perfect gas, the speed of sound is solely a function of temperature. Shock waves are mathematically modelled as infinitesimally thin region of highly viscous flow which creates a discontinuity in flow conditions.

Detonation waves are shock waves propagating through a combustible mixture. Detonation waves can either be initiated, e.g. by a spark plug, or generated through deflagration to detonation transition (DDT). In a DDT scenario, the deflagration combustion coalesces into a reaction front and accelerates to a sonic speed, causing a detonation wave to form. Detonation waves are made up of a combustion front attached to a shock wave. The instantaneous pressure gain across the shock wave causes the reactants to combust. Detonation waves were first recognized by two teams of researchers in the early 1880s; Berthelot and Vielle, and Mallard and Chatelier [20]. The latter 
pair demonstrated that deflagration can transition into a detonation in combustible mixtures. Soon after, Chapman established one-dimensional, steady theory for the propagation of a detonation wave. These efforts were expanded upon by Jouget and is now referred to as the Chapman-Jouget (CJ) theory. This theory is the simplest, as it considers the combustion reaction to occur instantaneously and completely across the detonation [21].

Later, in the 1940s, three researchers independently furthered detonation theory using similar methods. The contributions of Zeldovich, von Neumann, and Doring expanded understanding of detonation propagation. Their combined efforts yield the Zeldovich-von Neumann-Doring (ZND) model, comprised of a one-dimensional leading shock wave followed by a reaction front. In ZND theory, the shock wave compresses the reactants to a high-pressure state called the von Neumann spike (S) [20]. The mixture then undergoes reactions through the induction and heat addition zones. The induction zone considers the finite time required to initiate chemical reactions. This process is schematically presented in Figure 1.2.

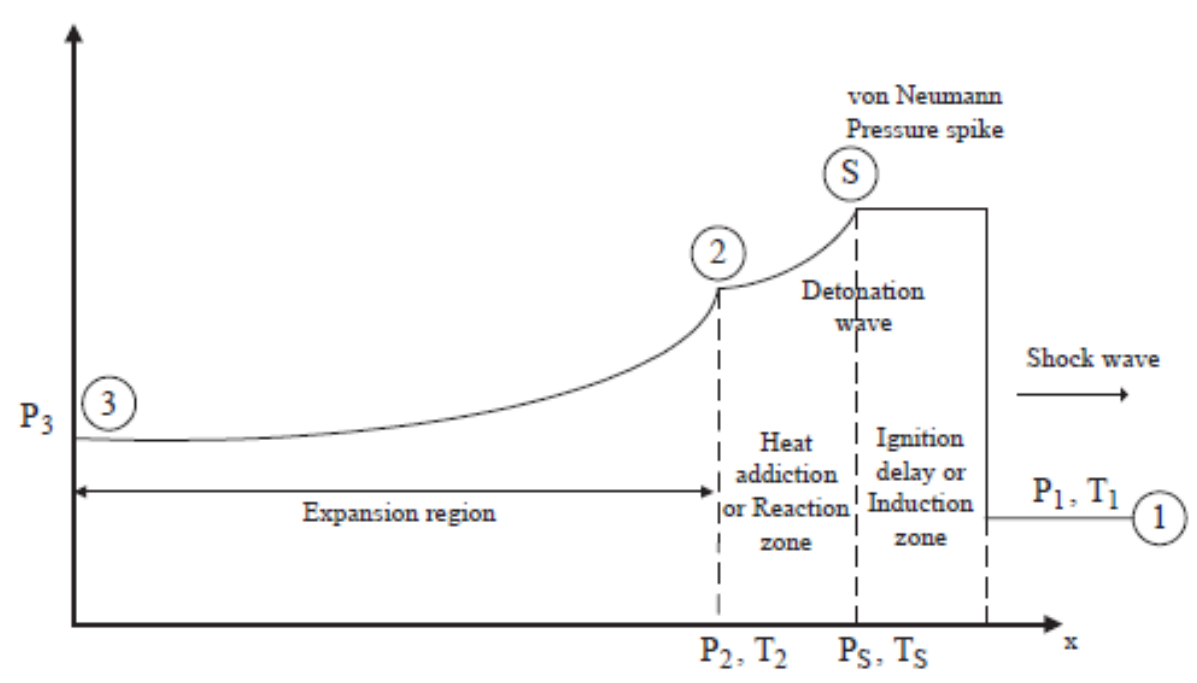

Figure 1.2: Detonation Pressure Profile under ZND Theory [20] 
In thermodynamics, pressure versus specific volume $(P-v)$ and temperature versus entropy $(T-s)$ diagrams are commonly used to depict thermodynamic processes. The P- $v$ diagram will appear later in this text, and are helpful to demonstrate the amount of work done or required by a process. This is determined by the area under the process curve. Figure 1.3 shows the $P-v$ diagram for a representative detonation wave. The Hugoniot curve represents all the possible final states of a combustion process from an initial state $(A)$ with pressure $P_{1}$, specific volume $v_{l}$, and a heat addition of $q_{1}$. The dotted lines are Rayleigh lines which present the process path. The vertical Rayleigh line represents a perfectly isochoric (constant volume) process and point $B$ marks its final state. The horizontal Rayleigh line represents a perfectly isobaric (constant pressure) process and point $C$ indicates its final state. The Hugoniot curve between points $B$ and $C$ are impossible final states to achieve under initial conditions, $A$. The point $D$ is known as the Upper Chapman-Jouget point (UCJ). Similarly, $E$ is known as the Lower Chapman-Jouget point (LCJ).

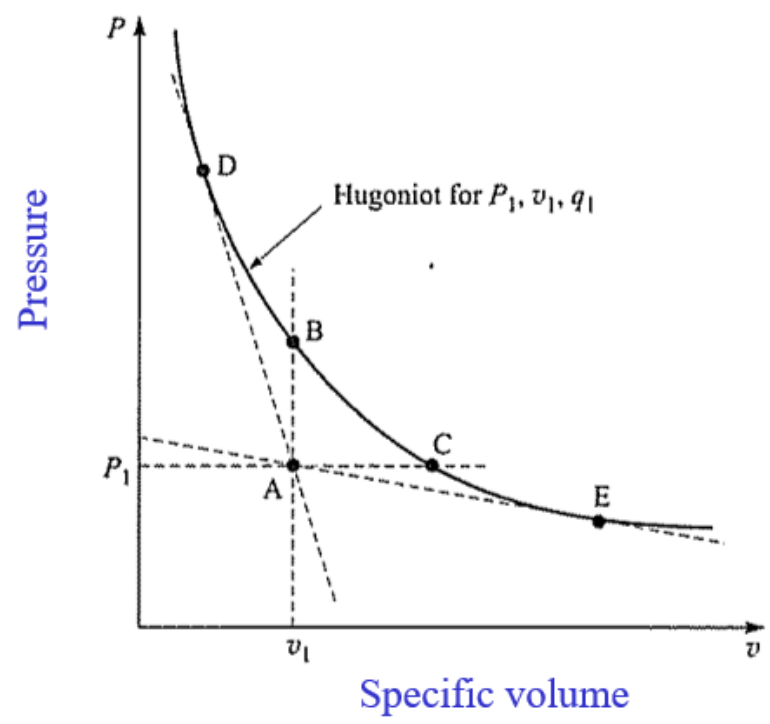

Figure 1.3: P-v Diagram for a representative detonative process [22]

The UCJ and LCJ points result from the two solutions from CJ theory. The Rayleigh line from AD depicts a detonative process, ending at the UCJ point where the detonation velocity relative to the combustion products is exactly sonic. The Upper CJ point gives a good estimation of 
detonations. In this work, the detonation velocity at the UCJ location is calculated and used to analyze the operational mode of an RDE. This methodology is presented in Section 4. A convenient tool for applying CJ theory to a gaseous mixture under given initial conditions is the Shock and Detonation Toolbox, openly distributed by the California Institute of Technology [23]. Analyses using the CJ velocity $\left(U_{C J}\right)$ are provided in Section 6. Note that using these theoretical approaches requires acknowledgement of non-idealities within the system being studied. Incomplete mixing, viscous losses, and mode-switching cause deviation from theory.

It was later demonstrated that detonation waves are typically characterized by unsteady, threedimensional structures which can only be represented by a 1D prediction in an averaged sense [24].

\subsection{Problem Statement}

Gas turbine operating efficiency has increased throughout recent decades. Thermal efficiency is primarily increased through the increase of compression ratio and turbine inlet temperature. Both of these parameters indicate increased potential to do work with the working fluid. As material temperature limits are met, interest is focused on compression ratio. Utilizing isochoric combustion enables a relative increase in compression ratio due to the elimination of pressure loss across the combustor. According to Richards [7], a 5-10\% gain in overall efficiency is realistic. For these reasons, it is clear that pressure gain combustion is an exciting and promising next step in the development of gas turbine engines for power generation and propulsion applications. NETL has recently focused efforts on RDE research, investigating operational regimes of RDEs as well as a complication known as backflow which will be discussed in the next section. This work will attempt to create dimensionless relationships among the work that NETL is performing. 


\section{Literature Review}

\subsection{Research Area Background}

The team at NETL is striving to better understand detonation combustion, specifically with applications to rotating detonation combustors. To achieve these goals, the team makes use of two experimental rigs, a lab scale RDE (LSRDE) and a bench scale RDE (BSRDE), in addition to computational fluid dynamics (CFD) models. The primary rig (LSRDE) is a 6-inch diameter rotating detonation combustor (RDC). The LSRDE utilizes the Air Force Research Laboratory (AFRL) inlet geometry. This rig is fully functional and is used to examine the impacts of preheat and back-pressure on RDC operability. The team is also trying to measure exhaust gas composition. While the LSRDE provides an accurate representation of the physical environment of an RDE, it is difficult to perform flow visualization studies due to the geometric complexities and extreme operating conditions of the facility.

In 2017, Roy, et al. [15] performed preliminary research on the LSRDE. By investigating equivalence ratio, air supply, fuel supply, air pre-heat, and exhaust back pressure effects on system stability, operational regimes and instabilities could be analyzed. It was found that increased air injection temperature and back pressure seemed to encourage higher order operating modes. Specifically, at increased air injection temperatures, the typical two-wave system bifurcated into a

three-wave mode. Additionally, the LSRDE was observed to operate in an unstable mode under lean mixture conditions.

More recently, Roy, et al. investigated the influence of natural gas addition to the fuel mixture in this facility [25]. In general, it was found to be challenging to sustain stable operation at higher contributions of natural gas. 
Since testing for these studies $[15,25]$ was completed, the LSRDE underwent alterations to enable similar studies with a larger detonation channel. The channel width was increased from $5.1 \mathrm{~mm}$ to $7.6 \mathrm{~mm}$. The data analyzed in the present work will be the first analysis on the wider channel.

The BSRDE has been constructed to facilitate learning in the areas of injection geometries, channel feedback, and inlet pressure losses. In this rig, Schlieren Imaging is used to observe combustion products travelling upstream into the fuel and air plenum.

The BSRDE has undergone several iterations of a geometrically similar, unwrapped RDC, operating at lower pressures and flow rates [26,27]. Preliminary research investigated a variety of air-inlet geometries. This work made use of Schlieren imaging to gain insight into combustion feedback and reflected shock behavior in the plenum. Though much of the early analyses were qualitative, they form the foundation of the current work [28]. Further efforts have witnessed investigations into three parameters: interruption time, recovery time, and backflow length. The latter two are determined through MATLAB schemes which attempt to analyze combustion products propagating through each frame of the Schlieren imaging data. Interruption time is defined in this, as well as previous works [29], as the time during which the measurement device is excited above steady state due to a detonation event. Graphically, this parameter is depicted in Figure 2.1. Recovery time is more difficult to obtain. It represents the duration of time in which combustion products exist within the injection plenum. For some geometries tested, the recovery time was infinite. Finally, backflow length is simply the furthest upstream distance that the combustion products reached relative to the injection plane. 


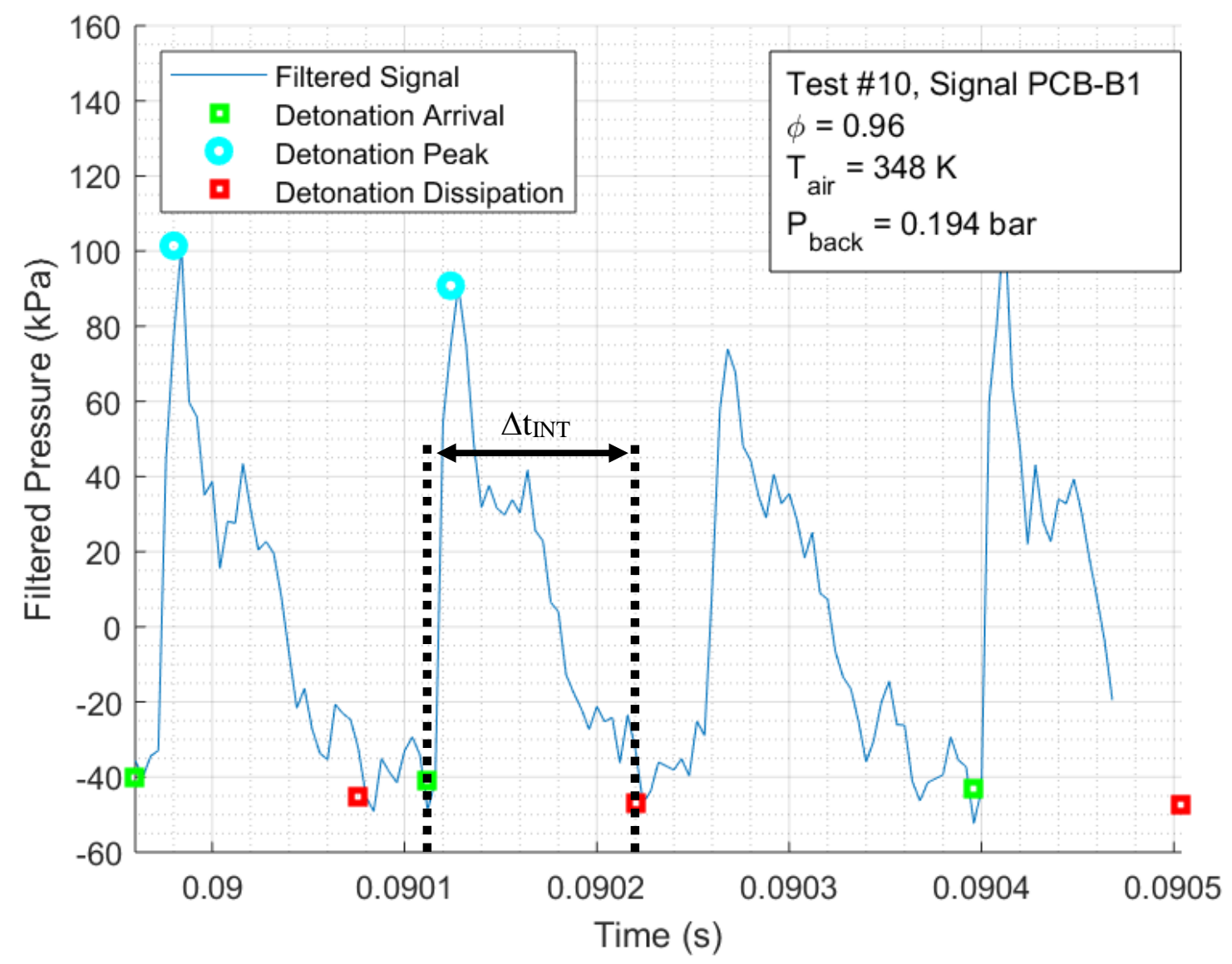

Figure 2.1: Visual Representation of Interruption Time

\subsection{Inlet Challenges}

Other research teams are working to resolve the general question of how to reduce feedback into plenums. In 2012, Braun, et al. [29] perform a parametric study of the effect of injection geometry, fuel type, and supply pressure on the performance of a fluidic valve. Here, fluidic valve takes on the general meaning of an injection geometry which employs a cavity between the reactant supply and the injection plane. This study was done using a linear detonation tube, which permitted a single shot study. A schematic of the laboratory setup is provided in Figure 2.2. 


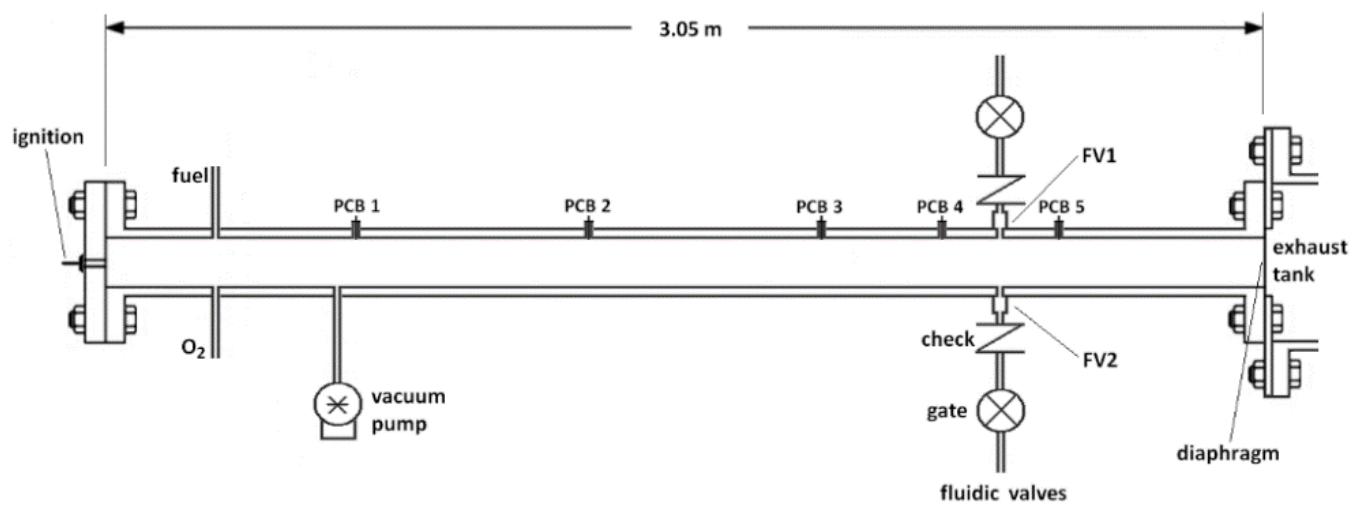

Figure 2.2: Laboratory Setup Schematic [29]

Braun, et al. define the interruption time as the time during which the dynamic pressure signal is excited above the steady state value. It is mentioned that this value is not the recovery time, which is defined as the time during which fresh reactants are not flowing through the injector. Braun, et al. suggests that CFD or optical access to the plenums of an experimental RDE is needed to further investigate the recovery time. The research team proposes two non-dimensional parameters, interruption time ratio $(\tau)$ and a dimensionless pressure $(\Pi)$. The two are defined as follows in equation (2.1).

$$
\begin{gathered}
\tau=\frac{t_{\text {int }}}{\left(L / U_{C J}\right)} \\
\Pi=\frac{\left(p_{P} / p_{0}\right)}{\left(p_{C J} / p_{0}\right)}
\end{gathered}
$$

Here, $\mathrm{L}$ is the distance from the ignition source to the valve orifice ("ignition" to FV1 and FV2 in Figure 2.2), $\mathrm{U}_{\mathrm{CJ}}$ and $\mathrm{p}_{\mathrm{CJ}}$ are the theoretical wave velocity and peak pressure based on ChapmanJouget theory, $\mathrm{p}_{\mathrm{P}}$ is the peak cavity pressure, and $\mathrm{p}_{0}$ is the initial pressure. Interruption time in this work is defined in the same way as in NETL literature. The results for varying the orifice geometry and the fuel type are provided in Figure 2.3. Note that the detonation that is studied is fueled by a propane and oxygen mixtures. The fuels presented in Figure 2.3 are surrogates that are injected 
through the fluidic valve to mimic an RDE application. It is apparent that the relationship between interruption time ratio and the dimensionless pressure term is generally linear, suggesting that the fluidic valve scales mainly with detonation pressure, injection pressure, and frequency of operation.

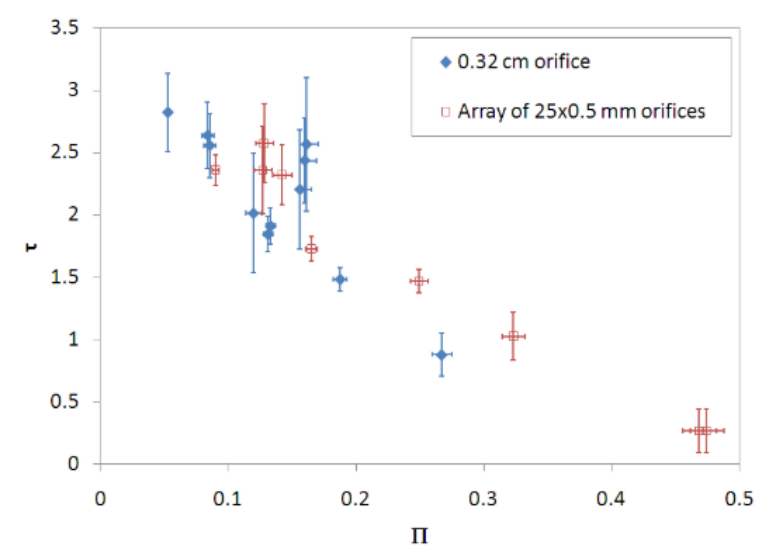

(a) Orifice geometry comparison.

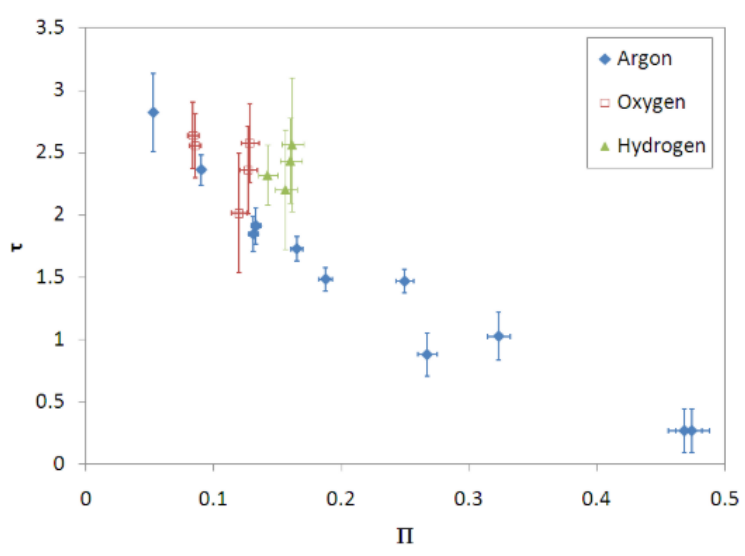

(b) Fuel comparison.

Figure 2.3: Plot of dimensionless terms for changing (a) geometry and (b) fuel type [29] The data also indicate that as supply pressure increases, $p_{P}$ increases and the interruption time decreases. This finding is more clearly illustrated in the plenum reflection diagrams in Figure 2.4.

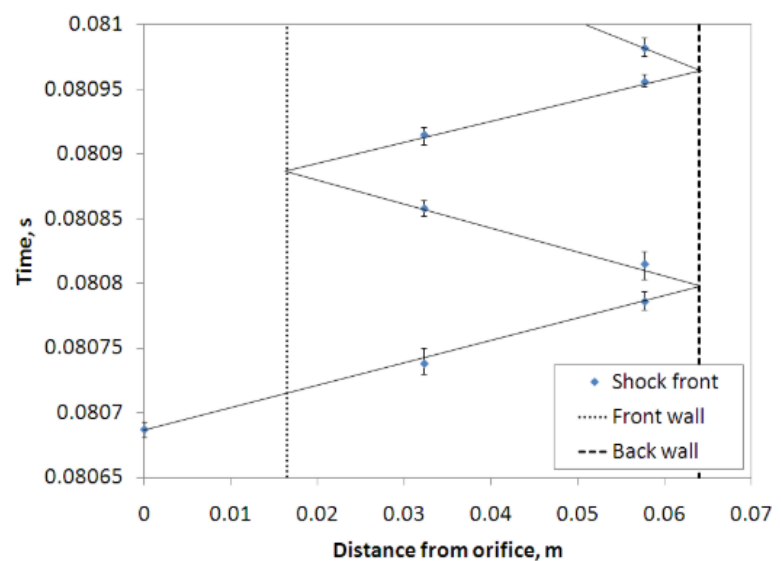

(a) $380 \mathrm{kPa}$ Injection Pressure

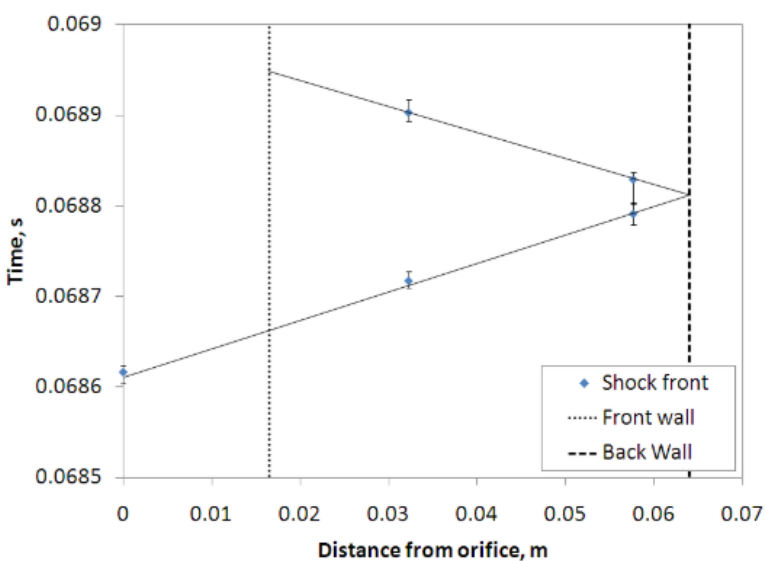

(b) $2300 \mathrm{kPa}$ Injection Pressure

Figure 2.4: $x$-t diagrams depicting shock reflections within cavity [29] 
Peace, et al. [30] experimentally investigated a variable high frequency fluidic valve mounted to a PDE. These experiments were quite similar to those of Braun, et al., however utilizing a PDE allows for analysis of cycle time and variability on fluidic valve performance. In the study, cavity length and injection pressure were varied to determine their impact on incident shock behavior within the valve plenum. It was found that the duration of the existence of reflected shock waves in the plenum cavity increased with air supply pressure, effectively disagreeing with the findings of Braun, et al. This is apparent from comparing the $\mathrm{x}$-t diagrams in Figure 2.5.

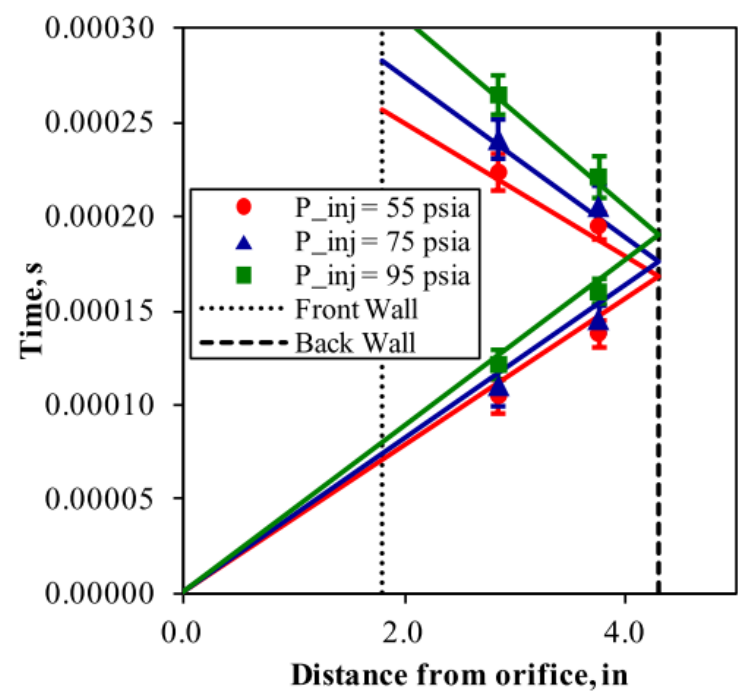

(a) 2.5 in. long cavity

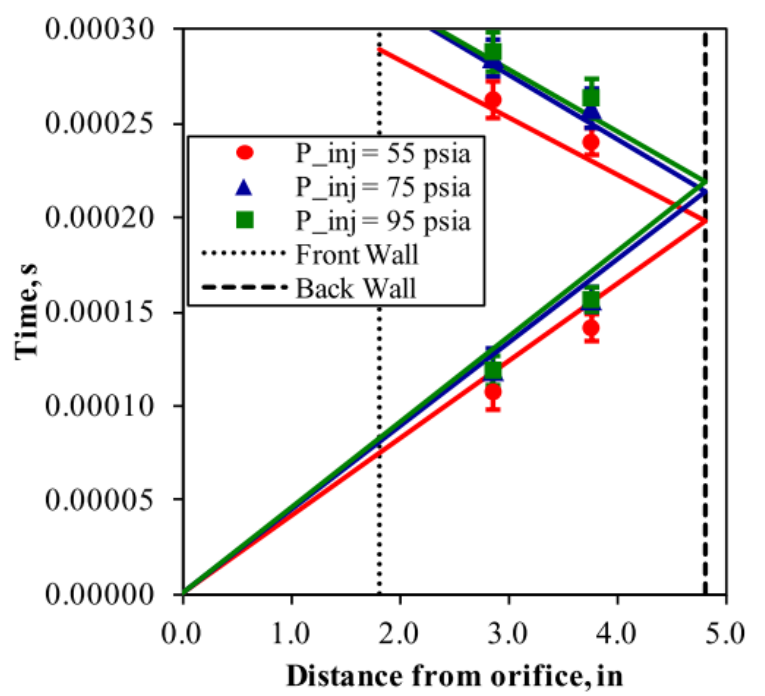

(b) 3 in. long cavity

Figure 2.5: x-t diagrams depicting shock reflections [30]

Naples, et al. [31] outfitted an operating RDE with ITP mounted PCBs and custom made hot film anemometers to enable further investigation into fuel plenum dynamics. The dynamic pressure measurement and hot film velocity measurement within the fuel plenum was compared to the response seen in the channel dynamic pressure measurement. It was found that the plenum response is related to the channel response in the form of harmonics. Each of the three measurement responses can be seen below at two different mass flow rates in Figure 2.6. 

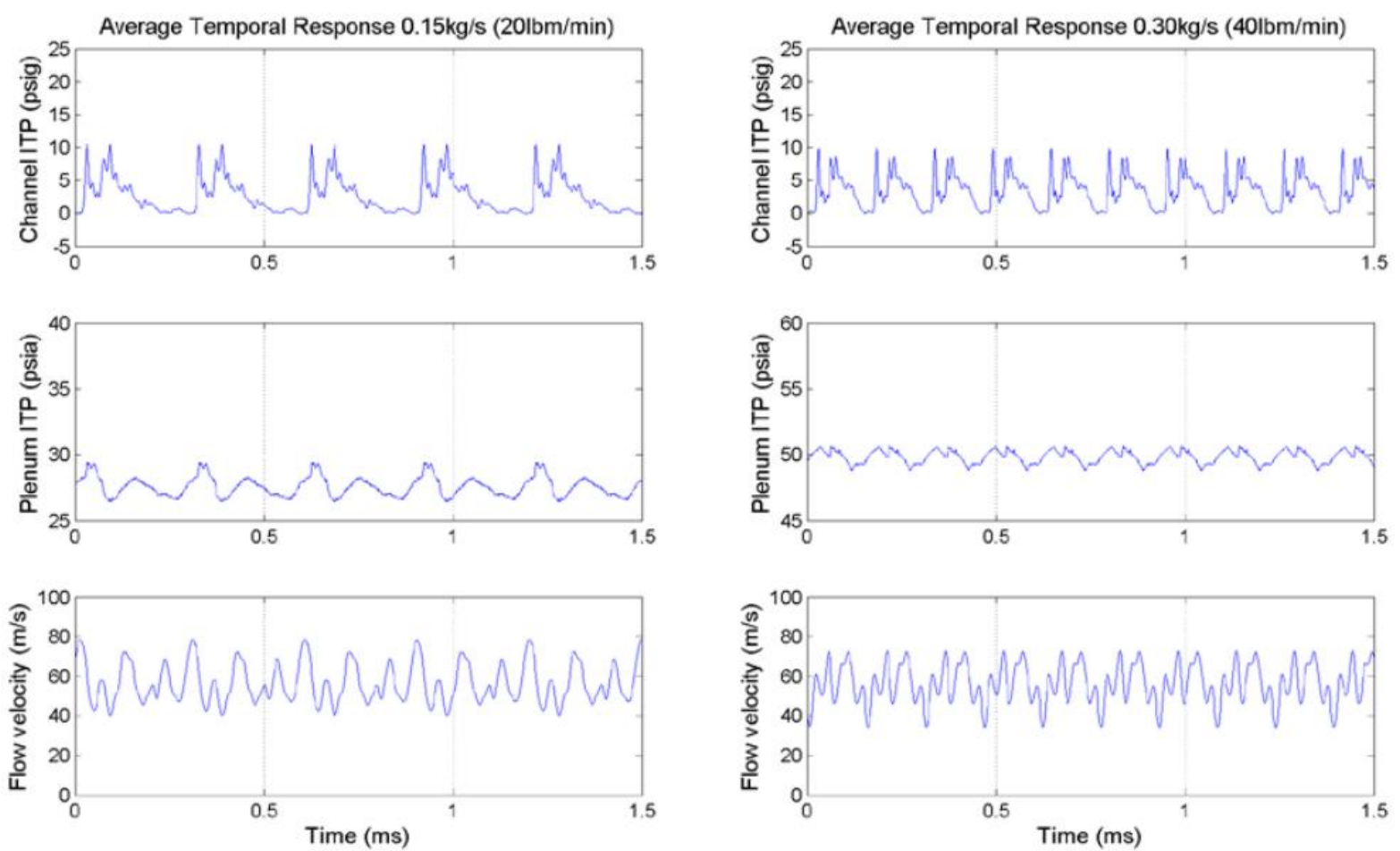

Figure 2.6: Signal Response for different measurement locations and types at mass flows of (left) $0.15 \mathrm{~kg} / \mathrm{s}$ and (right) $0.30 \mathrm{~kg} / \mathrm{s} \mathrm{[31]}$

The plenum response shows a high frequency component that likely results from geometric pressure reflections or flow disturbances, which can occur in the form of wave reflections, geometric natural frequency excitation, boundary layer interaction, or large-scale vorticity. A key finding was that by comparing the plenum velocity response to sonic nozzle metered flowrates, the mass flux through the plenum was found to be substantially higher, indicating that there is substantial error in the measurement technique. This finding also suggests that the high frequency fluctuations in the signal can be attributed to vorticity and boundary layer interactions, as both effects would not be discernible from measurement response, unlike natural frequency excitation and wave reflections.

At the University of Cincinnati's (UC) Gas Dynamics and Propulsion Laboratory, various RDC instabilities have been investigated, and initial operational maps and conclusions have been 
suggested [16]. The four instabilities discussed include chaotic instability, low-frequency waxing and waning of peak detonation pressures, mode switching, and longitudinal pulse detonation (LPD) operation. The first instability is postulated to be caused by lean operating equivalence ratio and low injection pressures, due to either larger injection orifices or lower mass flow. The second is likely to occur due to a low-frequency oscillation within the air inlet, which may be related to the Helmholtz frequency of the inlet geometry. The third condition does not have any clear causes, however this study suggests that a multi-wave operating mode may be more stable than a singular wave due to lower pressure peaks in the channel, likely resulting in less pressure feedback to the plenums. Finally, the LPD instability seems to result from subsonic air supply and elevated back pressure.

Of primary interest to the present work is the low-frequency waxing and waning of peak pressures. The data and associated indicators for this condition are shown below, in Figure 2.7, based on the work at UC. 

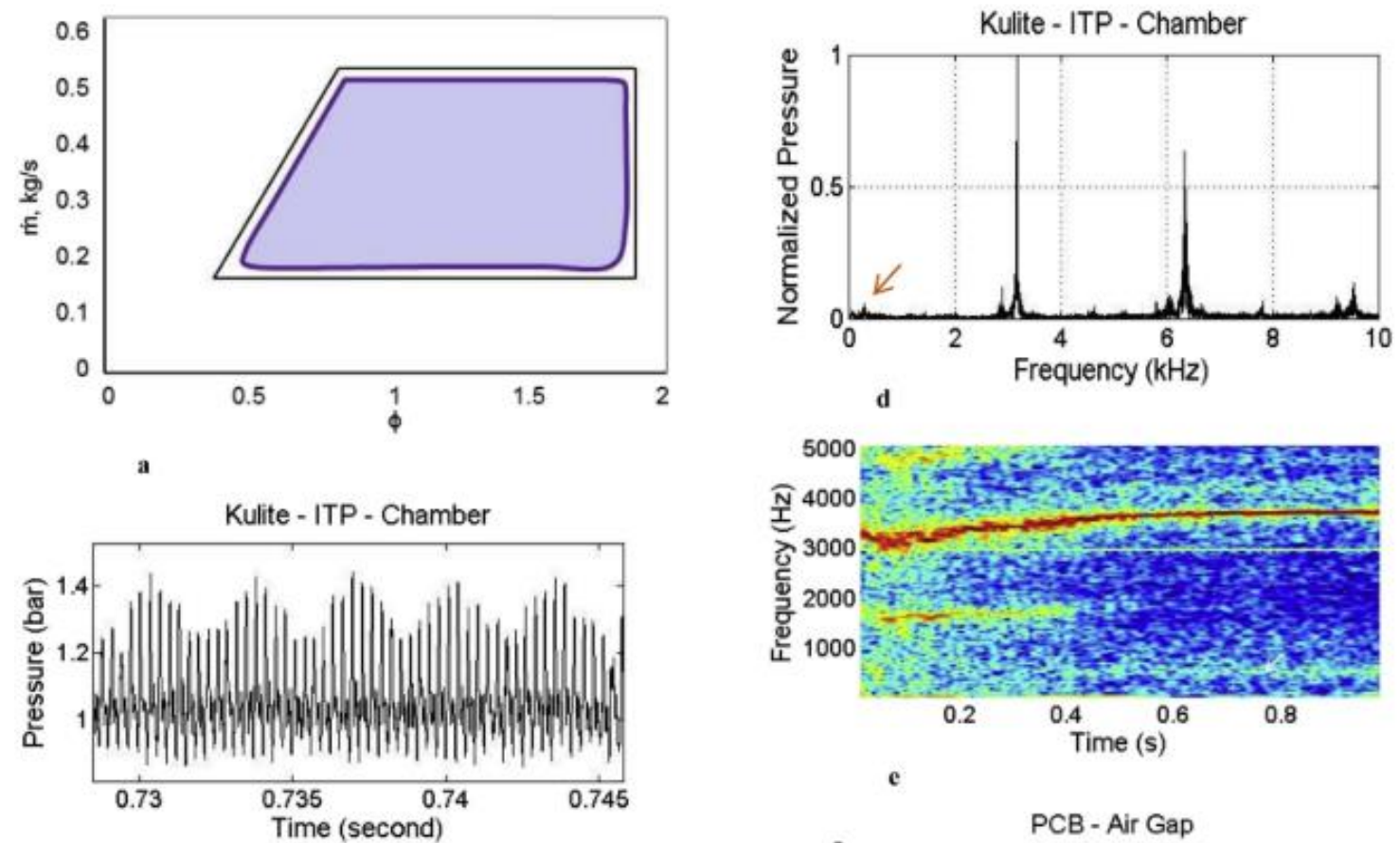

b
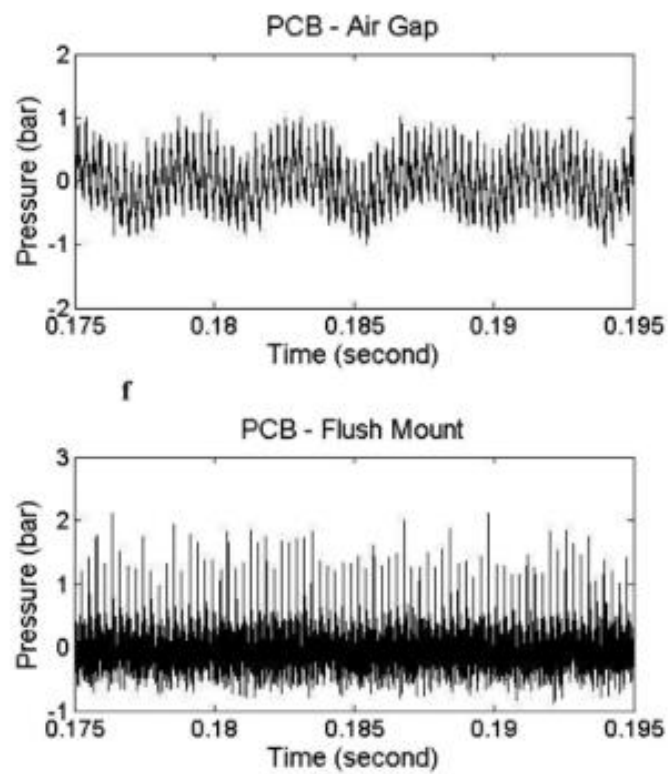

Figure 2.7: Operational Map and Indicators for LPD Condition [16]

In subsequent work, Anand, et al. [32] attempted to characterize the low-frequency oscillation discussed above. By investigating the FFT of air plenum PCB responses for stoichiometric cases at various air supply flow rates, the fundamental and secondary frequencies can be compared. These relationships are depicted in Figure 2.8. 


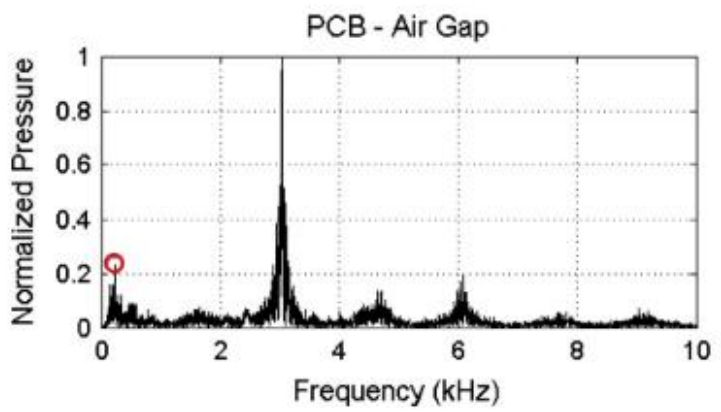

(a)

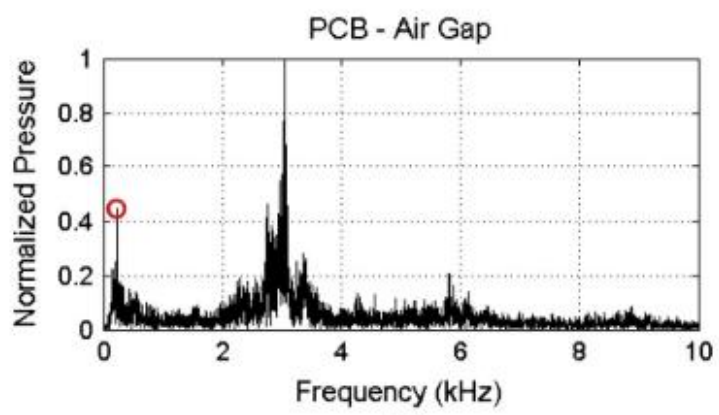

(b)

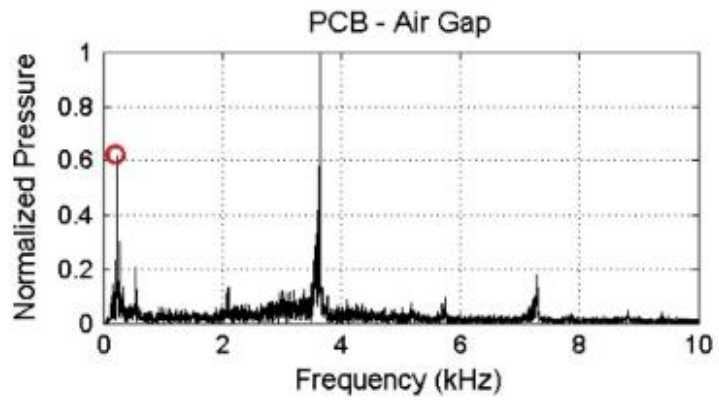

(c)

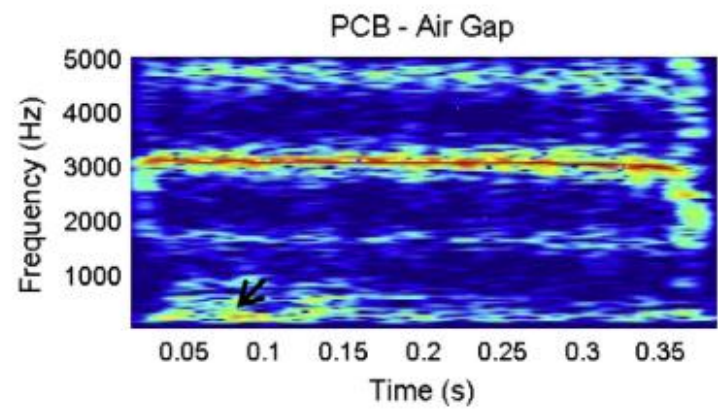

(d)

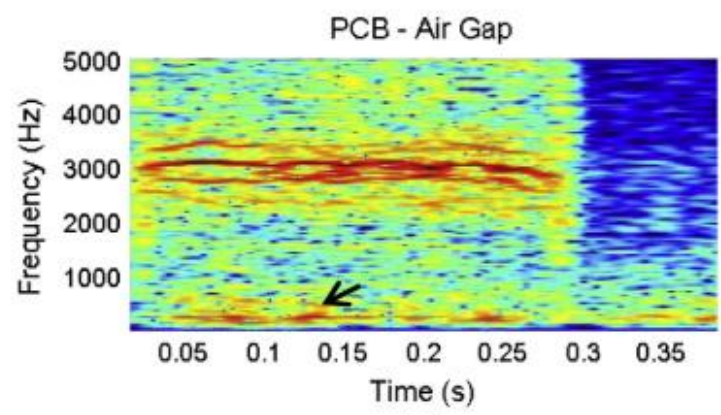

(e)

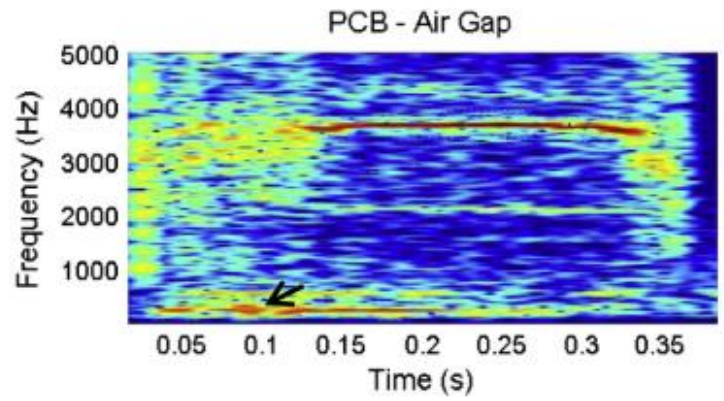

(f)

Figure 2.8: FFT and Spectrogram Comparison at $\phi=1.0$ and air supply flow rates of (top) 0.2 $\mathrm{kg} / \mathrm{s}$, (middle) $0.3 \mathrm{~kg} / \mathrm{s}$, and (bottom) $0.4 \mathrm{~kg} / \mathrm{s} \mathrm{[32].}$

As indicated by the red circles in the FFTs (Figure 2.8, a-c), each signal shows some activity around $235 \mathrm{~Hz}$. Further investigation into the Helmholtz Resonance Frequency for the air plenum reveals an estimation of $355 \mathrm{~Hz}$. This calculation is within $33 \%$ of the secondary frequency seen in the FFTs. The Helmholtz calculation cannot be made exact as the plenum geometry is not exactly represented in the equation. With consistent existence of the $235 \mathrm{~Hz}$ signal content regardless of flow rate, and the closeness to the estimated Helmholtz frequency, it is reasonable to 
conclude that the low-frequency oscillation seen in the measurement signal is due to the Helmholtz resonance excited by the detonation wave.

Schwer, et al. have worked to establish a reliable CFD code which can be used to learn about the inner workings of RDEs. In a recent journal article, the team investigated several micro-injector concepts, shown in Figure 2.9. These injectors were found to significantly impact the flow field, however, demonstrated a rise in plenum pressure oscillations [33,34].

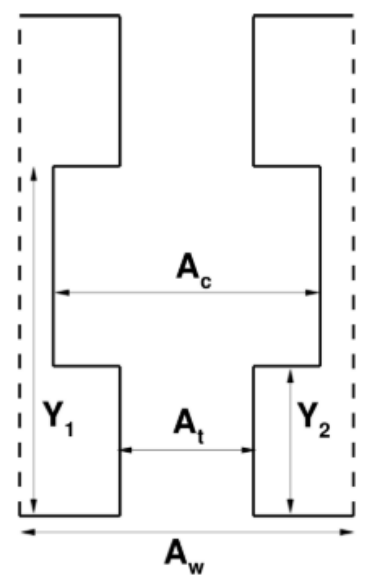

(a) Cavity-slot

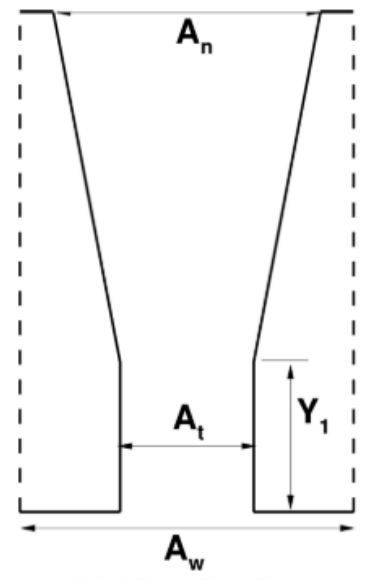

(b) Nozzle-slot

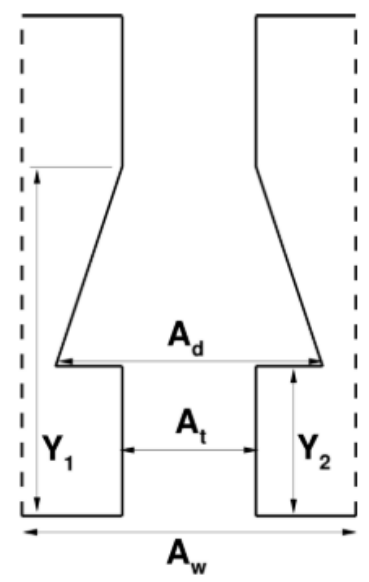

(c) Diode-slot

Figure 2.9: Schwer Injector Geometries [33]

Rankin, et al. have used mid-IR imaging on an optically accessible RDE to determine the detonation shape variation in response to changes in mass flow rate and equivalence ratio. It was concluded that the detonation wave height is initially increased with an increase in air mass flow rate, remains relatively unchanged with another increase in flow rate, and decreases at the highest air mass flow rate tested. The decrease is due to the mode change associated with the higher flow rate. Two-wave modes allow less fill time, and therefore smaller detonation waves. It was also found that minimal mixing between fresh reactants and combusted products from the previous cycle is occurring. This is discerned from the negligible $\mathrm{H}_{2} \mathrm{O}$ radiation emissions in front of the detonation waves [35]. 


\subsection{Pressure Measurement Techniques}

One potential source of discrepancy between the LSRDE and BSRDE experiments is pressure measurement techniques. In the BSRDE, flush mounted PCB transducers are used to capture dynamic pressure. The LSRDE operates at much higher temperature. Due to the high heat flux, transducers are mounted in an infinite tube pressure (ITP) arrangement. Gejji, et al. at Purdue have begun investigation into how these different transducer installations can impact the pressure measurements obtained. They found that the ITP arrangement results in a $\sim 6 \mu$ s delay as well as an amplitude attenuation of 36-54\% [36]. It is widely accepted that PCBs are reliable for temporal response, thus it is interesting that the offset of the ITP causes such a delay. These findings do not cause irreparable error in experiment, but the actual time delay is important to know during data analysis. From Figure 2.10, the time delay can be visualized. Notice that a PCB mounted in an offset arrangement does not see anywhere near the changes that the ITP arrangement witnesses.

Naples, et al. performed a similar investigation [37]. In this work, transducer standoff, tubing length, tubing diameter, shock speed, gas species, and geometry were varied to shed light on ITP installation. It was found that the shock speed within the ITP consistently correlated closely to a Riemann problem solution, indicating that the shock present in the tubing occurs due to the sudden pressure rise. Tubing diameter was determined to have significant effect on measurement. Smaller diameter tubing unsurprisingly increase viscous effects. Larger diameter tubing produce more consistent results, but also allow more heat to come into contact with the transducer and vents more mass from the measurement device. Of most significance was the effect of cavity volume. Large cavities were found to have time lags of as much as 500 microseconds. 


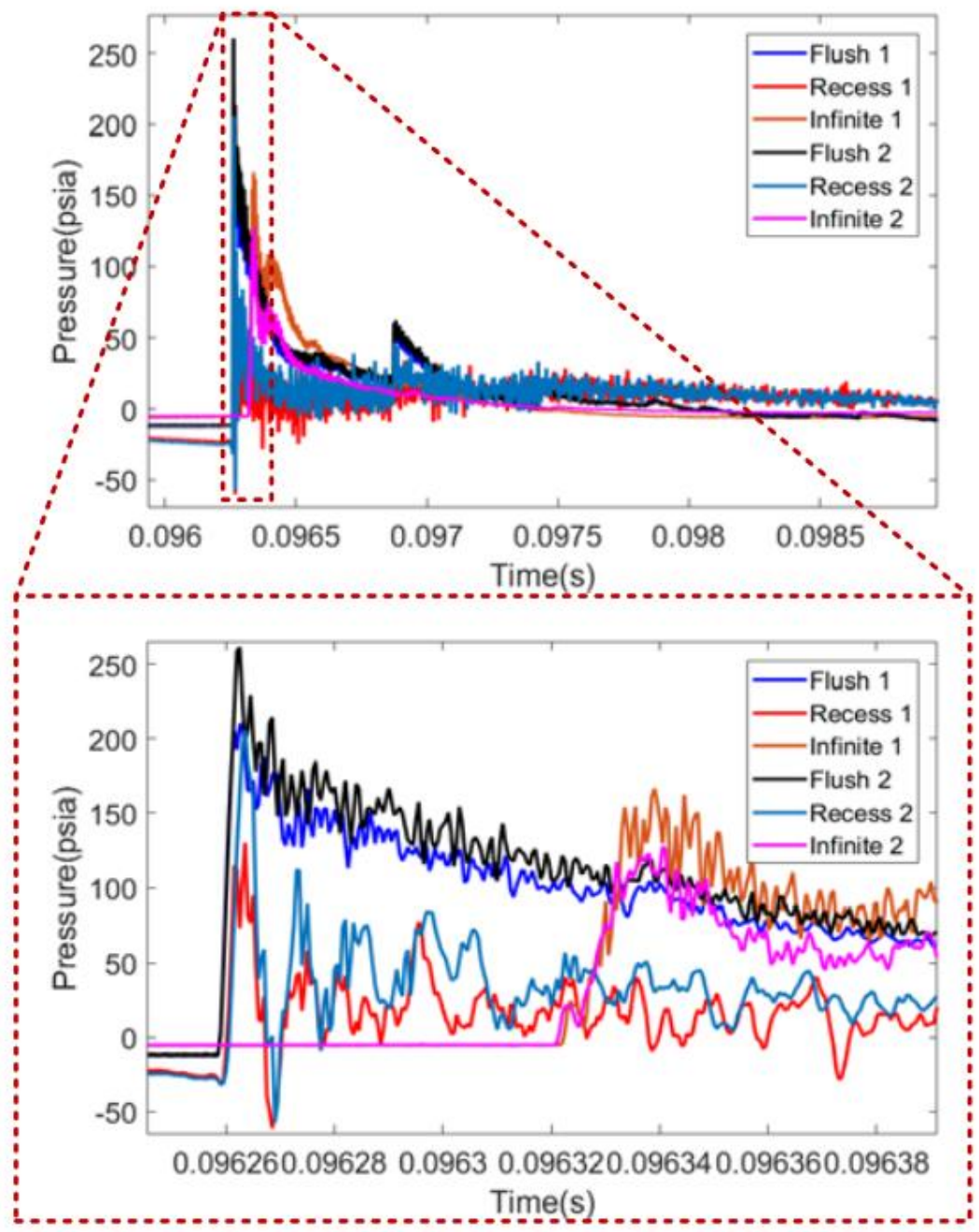

Figure 2.10: Gejji PCB results [36]

\subsection{Present Work}

The primary desire for the results of the BSRDE is to serve to direct modifications to the LSRDE. Issues in developing a relationship between the two experimental rigs revolve around the disparities in flow mechanisms. Details regarding each rig, their geometries and operational capabilities, will be discussed in Section 0.

The present work attempts to, firstly, determine significant non-dimensional parameters which define the RDC environment, and secondarily, create relationships between the two NETL operated experimental rigs. This is accomplished via a dimensional analysis using the Buckingham 
Pi theorem, selecting a dimensionless time and pressure term to investigate further, and manipulating data for both rigs to work toward parameters which can be compared directly. Namely, this study focuses on an interruption time ratio and dimensionless pressure. The interruption time ratio is defined as the ratio of the time during which the dynamic pressure is excited above the unperturbed state due to a detonation event and the period of the RDC operation under the specific test conditions. The period is found via the dominant frequency as found in a Fast Fourier Transform (FFT) of the system. In other words, the interruption time ratio is the percentage of the RDE period during which the pressure is above steady state due to a detonation. The pressure term is made dimensionless by the air injection gap height, supply air density, and supply air flow rate.

\section{Experimental Setup}

\subsection{LSRDE Experimental Setup}

NETL's lab scale RDE is based on the AFRL 6 inch diameter rig design [38]. The facility maintains the internal geometry of the AFRL design, but is integrated into the NETL laboratory such that the RDC can be tested at elevated pressure and temperature. The Low Emissions Combustion Test and Research (LECTR) facility, Figure 3.1, allows the RDC to operate in a closed flow configuration with a back pressure control valve. The rig is limited to running for 6-10 seconds to prevent overheating due to lack of cooling strategies. Operational pressures reach up to 1.76 MPa. Supply flow rates are measured by orifice meters and controlled with valves. Air can be supplied to the rig at a maximum flow rate of $1.15 \mathrm{~kg} / \mathrm{s}(\sim 65,000$ SLPM $)$ and a maximum preheat temperature of $425^{\circ} \mathrm{C}$. 


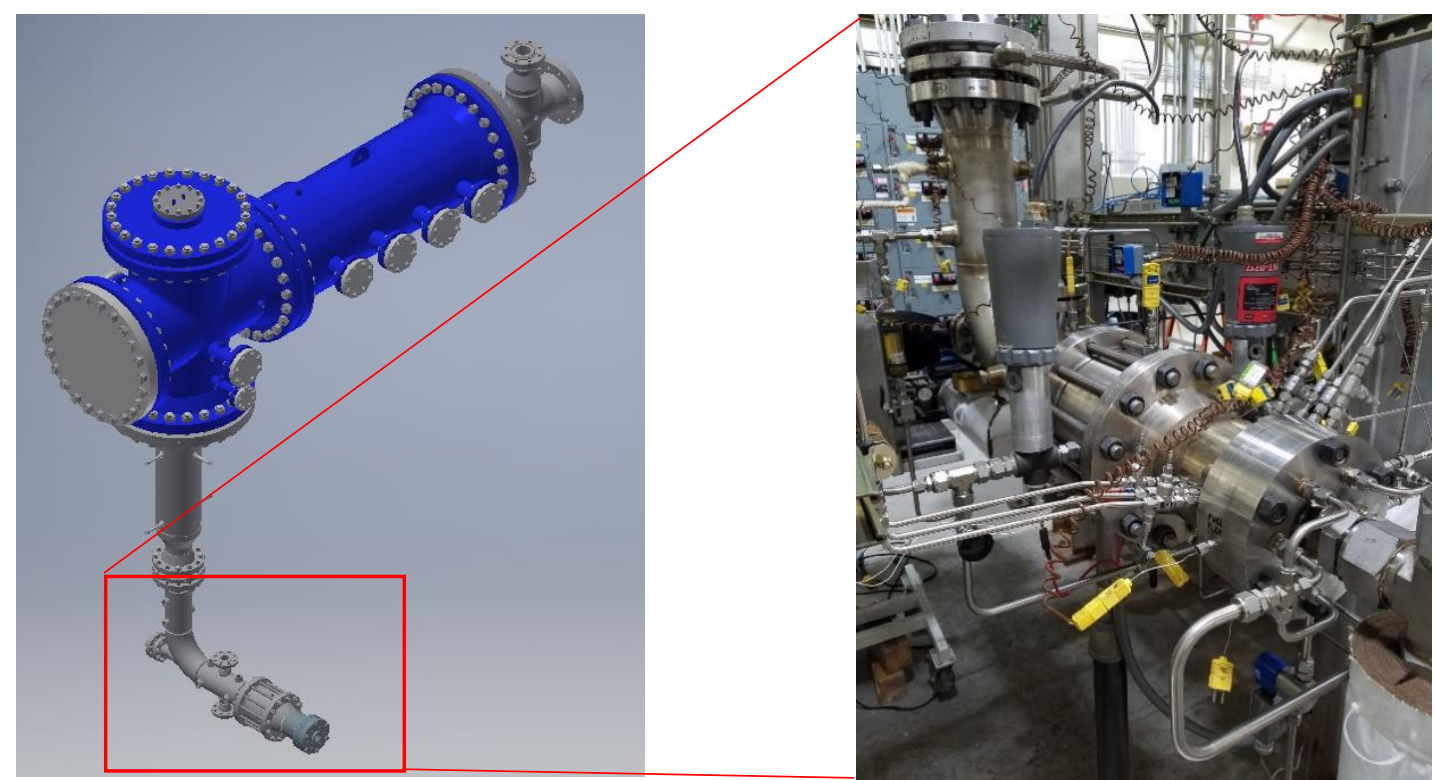

(a) CAD of LECTR facility

(b) Image of instrumented LSRDE

Figure 3.1: NETL LECTR facility

During testing, pre-heat temperature was limited to $200^{\circ} \mathrm{C}$ to protect instrumentation. Hydrogen or natural gas are supplied as fuel with maximum flow rates of $55 \mathrm{~g} / \mathrm{s}(37,500 \mathrm{SLPM})$ and $13 \mathrm{~g} / \mathrm{s}$ (9,750 SLPM), respectively. Hydrogen serves as the main fuel for this facility, however, natural gas is mixed in during some testing [15].

Figure 3.2 presents the cross-sectional geometry of the NETL LSRDE. Fuel is injected through an array of axially oriented, cylindrical injectors located radially inward of the detonation channel to promote mixing. Air is injected through an azimuthally continuous slot directed radially outward. Supply air is forced to make a $90^{\circ}$ turn in order to discourage backflow into the air plenum. This also results in a static pressure loss. 


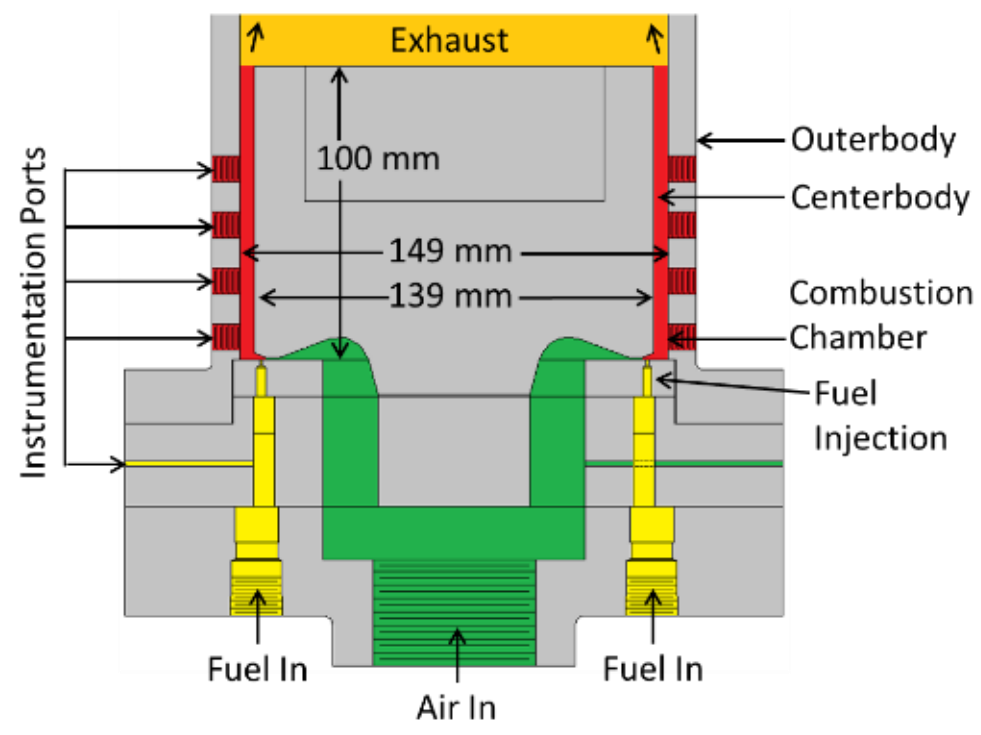

Figure 3.2: LSRDE Cross Sectional Geometry

Table 3.1 presents the instrumentation used within the LSRDE facility. An assortment of dynamic pressure, static pressure, and temperature measurement devices permit in depth investigation into the temporal behavior of the LSRDE in addition to heat transfer within the system. The following diagram, Figure 3.3, physically demonstrates where these various measurement devices are installed.

Table 3.1: LSRDE Instrumentation and Data Acquisition

\begin{tabular}{|l|l|l|l|}
\hline Port\# & Measurement & Sampling Rate & Instrument \\
\hline I1/I2 & Dynamic Pressure & $250 \mathrm{kHz}$ & PCB (ITP) \\
\hline A1/A4 & Static Pressure & $250 \mathrm{kHz}$ & Kulite (CTAP) \\
\hline B1/B2/B4/C1 & Dynamic Pressure & $250 \mathrm{kHz}$ & PCB (ITP) \\
\hline C2 & OH* signal & $250 \mathrm{kHz}$ & Optical Fiber \\
\hline C4 & Static Pressure & $250 \mathrm{kHz} / 2 \mathrm{~Hz}$ & Rosemount PT \\
\hline Fuel/Air Plenum & Dynamic Pressure & $250 \mathrm{kHz}$ & PCB \\
\hline Initiator Fuel & Dynamic Pressure & $250 \mathrm{kHz}$ & PCB \\
\hline Exhaust-1/2 & Dynamic Pressure & $100 \mathrm{kHz}$ & Kistler \\
\hline D1/D4-1-3 & Wall Temperature & $2 \mathrm{~Hz}$ & K Type TC \\
\hline
\end{tabular}




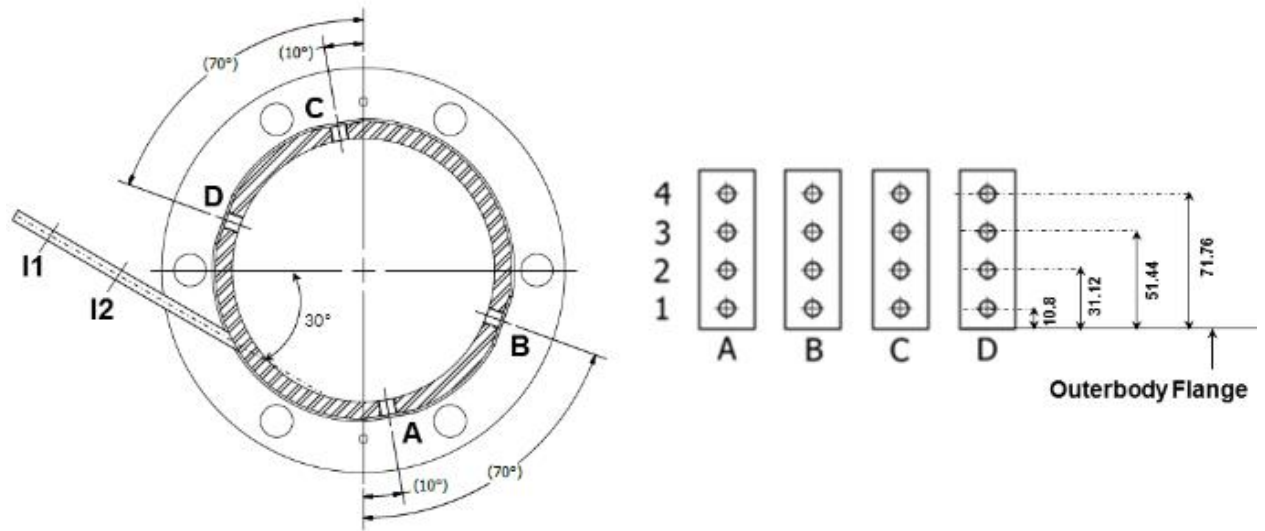

Figure 3.3: LSRDE Instrumentation Map

As is apparent in Figure 3.3, various azimuthal and axial measurement locations are employed, specifically for use with dynamic pressure transducers. This strategy enables a more complete picture of RDC operation, permitting comparison of pressure signals to analyze wave number and directionality.

\subsection{BSRDE Experimental Setup}

The BSRDE facilitates flow visualization access in the RDC channel and plenum. By linearly extruding the cross-sectional geometry of the LSRDE displayed in Figure 3.2, and again in Figure 3.4a for convenience, inlet dynamics and response can be more intimately studied.

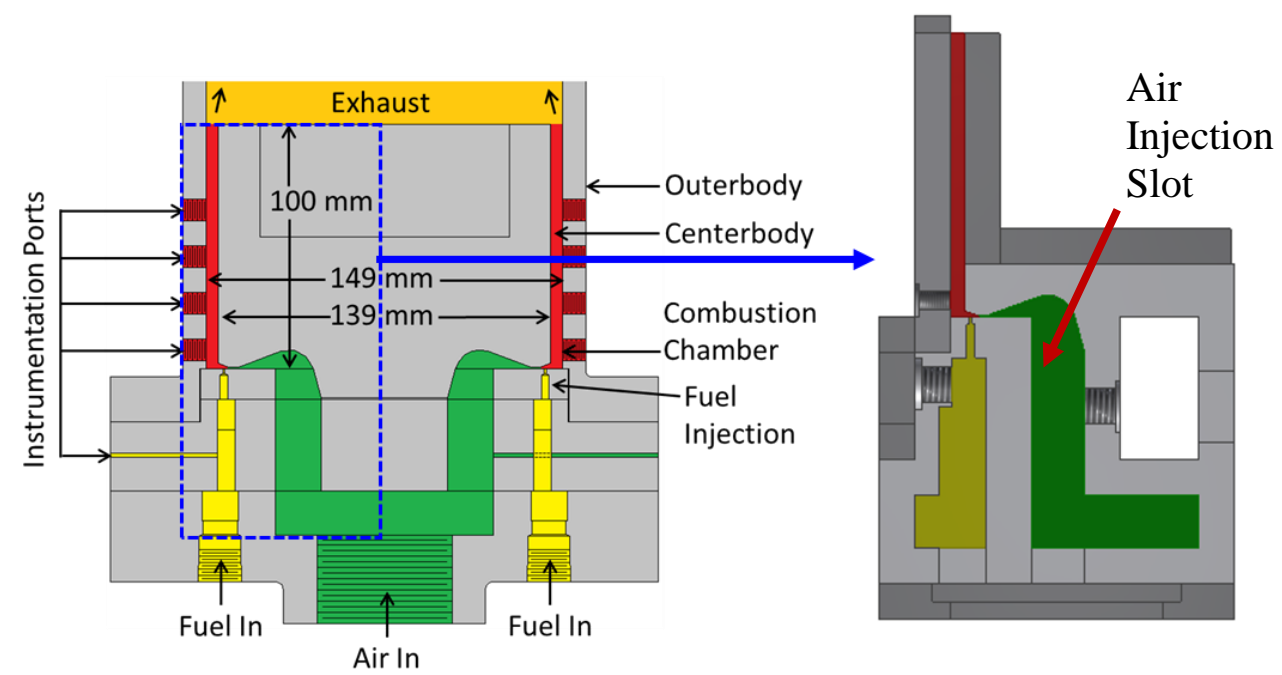

(a) LSRDE Cross Section

(b) BSRDE Cross Section

Figure 3.4: BSRDE cross sectional geometry 
The facility employs an aluminum housing which holds the linearly extruded RDC. The housing was meticulously designed to enable as much optical access as possible. Thus far, only the longitudinal optical access has been utilized as it is most helpful in viewing plenum and channel interactions. This view is comparable to that of Figure 3.4(b). Each test article is 3D printed using a Stratasys Object Eden 260VS printer and VeroClear material. This enables rapid prototyping to investigate various geometries. The aluminum housing is portrayed in Figure 3.5.
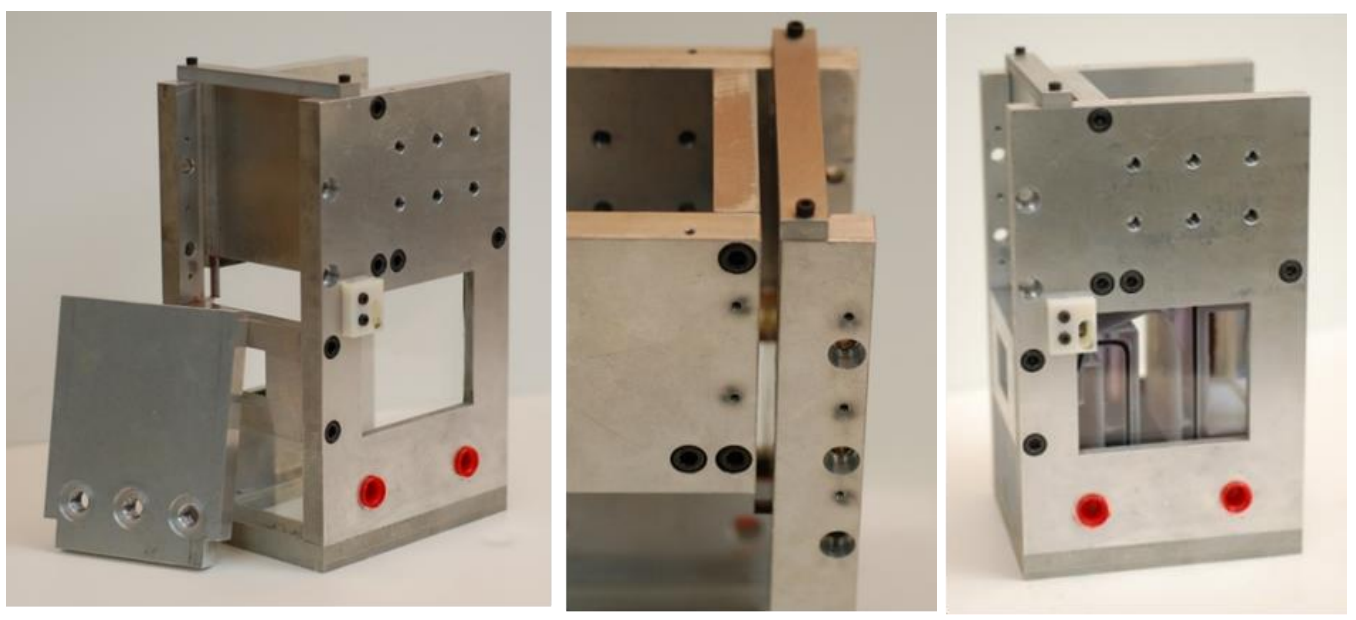

Figure 3.5: Aluminum housing and 3D printed reference inlet

The reference inlet (Figure 3.6), used extensively in this study, resembles the geometry first proposed by the Air Force Research Laboratory and is also used in NETL's LSRDE. The inlet has a circumferential air injection gap of $0.55 \mathrm{~mm}$. Fuel is not currently used in this rig, however, can be injected axially via an array of injection orifices. 


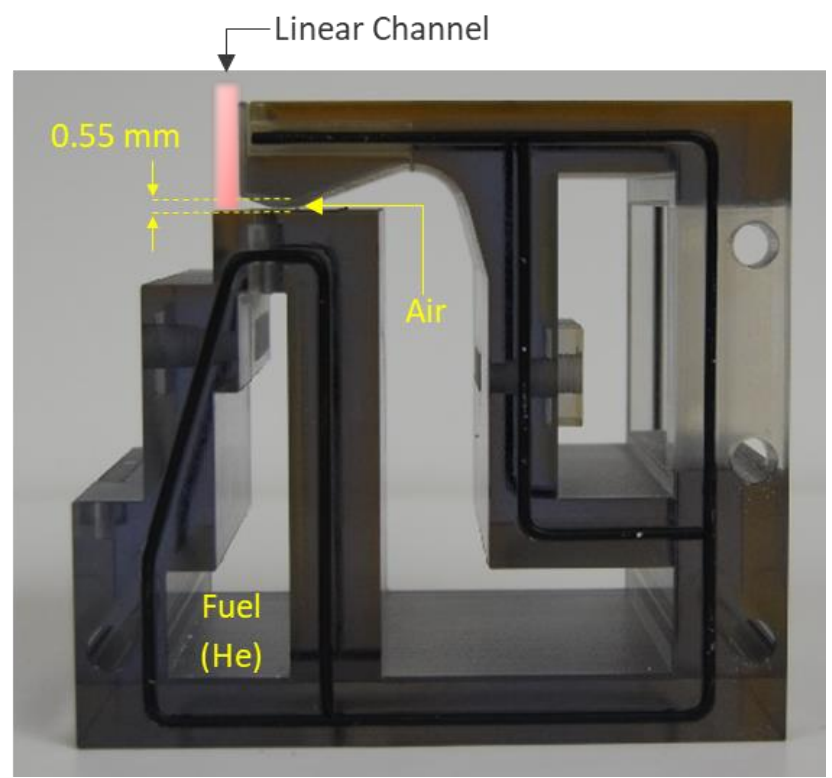

Figure 3.6: BSRDE Reference Test Article

The test article is anchored and outfitted with 1-3 Piezoelectric PCBs mounted in the outerbody wall to monitor the detonation events. A PCB is also placed in each plenum, in addition to a static pressure probe. Feeding the experiment is a $1 / 4$ " Swagelok pre-detonator tube with a split path fitting to enable a simulated wave period. The pre-detonator tube is supplied with hydrogen and air. A spark ignitor initiates a detonation event, which propagates through the tube and expands into the test section. The relative layout of this equipment is seen below in Figure 3.7.

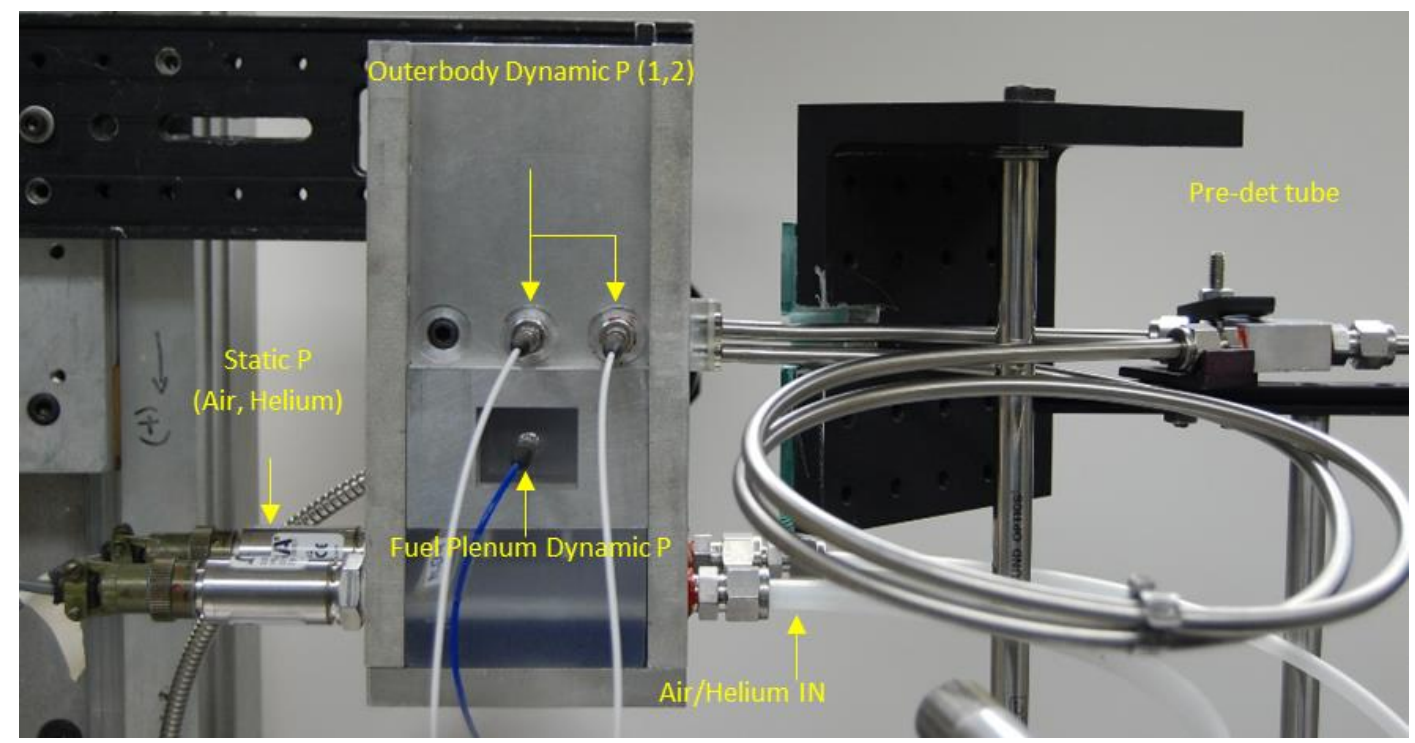

Figure 3.7: Picture of BSRDE facility instrumentation 
The primary purpose of this experimental rig was to provide optical access to channel and plenum dynamics. Schlieren imaging is the selected flow visualization technique for this facility. The layout of the imaging equipment is provided in Figure 3.8.

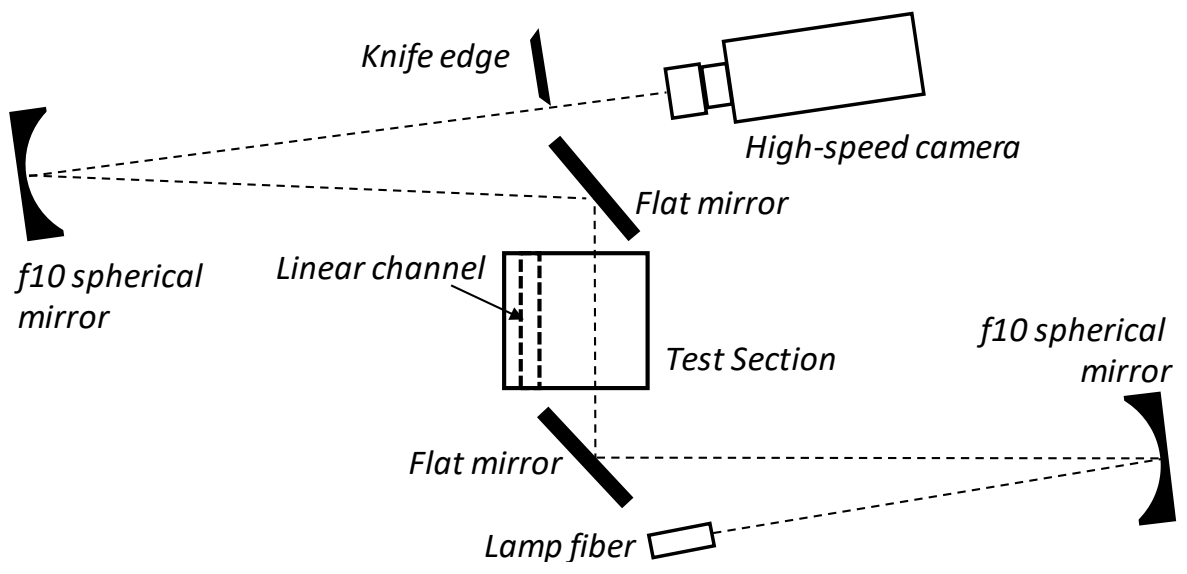

Figure 3.8: Schematic of Schlieren Imaging setup

Initial proof of concept tests and operational domain tests required the use of a considerable number of PCB transducers, labelled A-G in Figure 3.9. With this instrumentation strategy, the repeatability of the system can be tested, as well as the performance of the split-path tube and system performance in general.

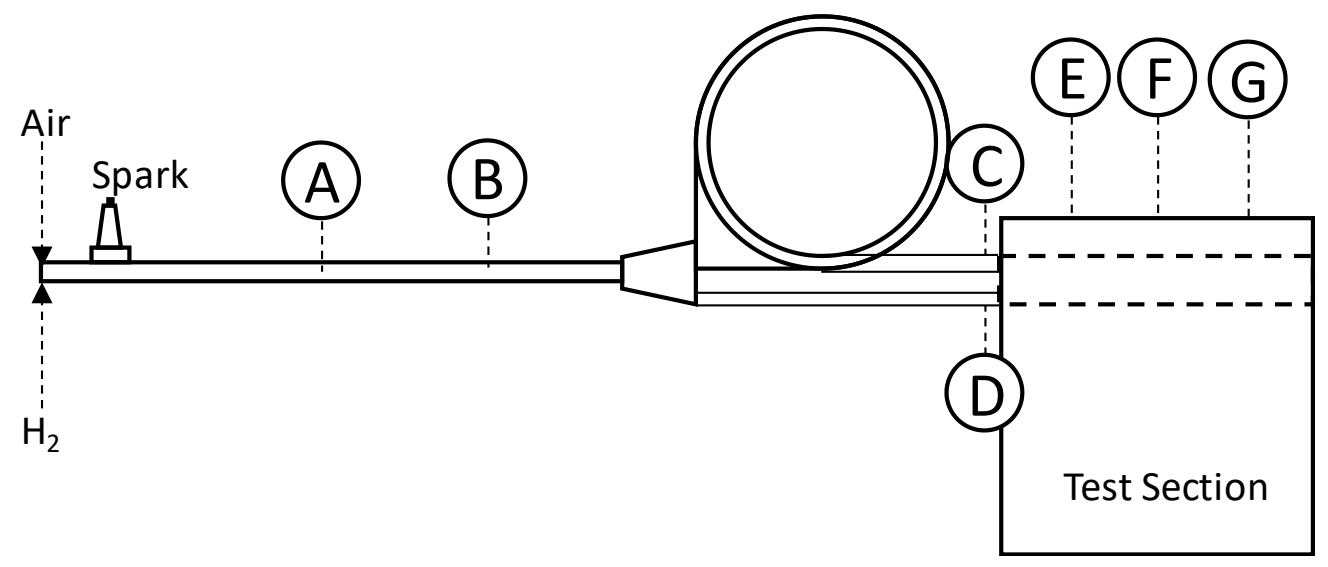

Figure 3.9: BSRDE Instrumentation Schematic 


\section{Methodology}

The following section includes the methods and strategy used to investigate the RDC environment, and more specifically, the two rigs at NETL. First, the methodology for the bench scale rig is presented, beginning with a dimensional analysis on the RDC system. Then, the LSRDE data reduction is presented.

\subsection{BSRDE Methodology}

In order to derive comparable parameters with the LSRDE, a dimensional analysis is performed. The results of the analysis are considered and two parameters are selected to be further investigated, namely, the interruption time ratio and a dimensionless pressure term. The methods used to reduce data into these forms are then presented and discussed.

\subsubsection{Dimensional Analysis and Buckingham Pi Theorem}

In a system of interest, the desired dependent variable can be a function of several independent variables. Examining the influence of each independent variable on the system may be unrealistic due to time and cost. By performing dimensional analysis via the Buckingham Pi Theorem, the same problem can be reduced to a few non-dimensional parameters, significantly simplifying the experimental process. In the Buckingham Pi Theorem, the independent and dependent variables, are considered and collected for a given system.

$$
\text { Dependent Variable }=f(\text { Independent Variables })
$$

It is possible to perform Buckingham Pi using mass, length, and time $(M L T)$ or force, length, and time $(F L T)$ as fundamental dimensions. Note that there are four fundamental dimensions; the fourth being temperature which is excluded from this fluid mechanical study. Each variable is then broken up into the aforementioned fundamental dimensions. The number of dimensions needed to represent the system is denoted ' $r$ '. This is also the number of repeating variables. The total number 
of variables, both dependent and independent, is denoted ' $k$ '. The difference between ' $k$ ' and ' $r$ ' represents the number of $\Pi$ terms, ' $n$ '. Repeating variables are chosen to represent each basic unit.

$$
\begin{gathered}
n=k-r \\
\Pi_{1}=f\left(\Pi_{2}, \Pi_{3}, \ldots, \Pi_{n}\right)
\end{gathered}
$$

Each $\Pi$ term is made up of the repeating variables and one other variable. In Equation (4.4), ' $q$ ' represents an independent variable. Assuming $r=3$ and utilizing the MLT fundamental dimensions, the following procedure is performed.

$$
\begin{aligned}
& \Pi_{i}=q_{i} \cdot q_{r 1}^{a} \cdot q_{r 2}^{b} \cdot q_{r 3}^{c} \\
& \Pi_{i} \doteq\left(M^{i 1} L^{i 2} T^{i 3}\right)\left(M^{r_{11} * a} L^{r_{12} * a} T^{r_{13} * a}\right)\left(M^{r_{21} * b} L^{r_{22} * b} T^{r_{23} * b}\right)\left(M^{r_{31} * c} L^{r_{32} * c} T^{r_{33^{*}}}\right) \\
& M: \quad i_{1}+r_{11} * a+r_{21} * b+r_{31} * c=0 \\
& L: \quad i_{2}+r_{12} * a+r_{22} * b+r_{32} * c=0 \\
& T: \quad i_{3}+r_{13} * a+r_{23} * b+r_{33} * c=0
\end{aligned}
$$

Through this process, several parameters have been discovered and have become famous due to the variety of applications in which they hold significance. Four such parameters include the Reynolds Number, Strouhal Number, Euler Number, and Cauchy Number. These parameters are often seen as ratios of significant influences on a system and are provided in Table 4.1.

Table 4.1: Common Dimensionless Terms

\begin{tabular}{|c|c|c|c|}
\hline Reynolds & Strouhal & Cauchy & Euler \\
\hline$R e_{c}=\frac{\rho U_{\infty} x}{\mu_{\infty}}$ & $S t=\frac{\omega l}{U_{\infty}}$ & $C a=\frac{\rho U^{2}}{E_{V}}$ & $E u=\frac{p}{\rho U^{2}}$ \\
\hline
\end{tabular}

The Reynolds number compares inertial and viscous forces in a given fluid. It is by far the most famous. The Strouhal number relates local and convective inertial forces. Compressibility forces and inertial forces are compared in the Cauchy number. Finally, the Euler number compares 
pressure forces to inertial forces. These four parameters are very common in fluid systems. Each was considered in the analysis of the experimental systems explored in this document. It was found that none of these parameters are directly applicable to the systems analyzed, however in the next section, the Buckingham Pi theorem is performed on the RDE environment and a new set of dimensionless variables are considered.

\subsubsection{RDC Dimensional Analysis}

A dimensional analysis was performed on the RDE system in order to investigate relationships between the two experimental rigs. Though the BSRDE and LSRDE differ greatly in the magnitudes of parameters and the detonation dependence on supply flows, they can both be represented by the following drawing.

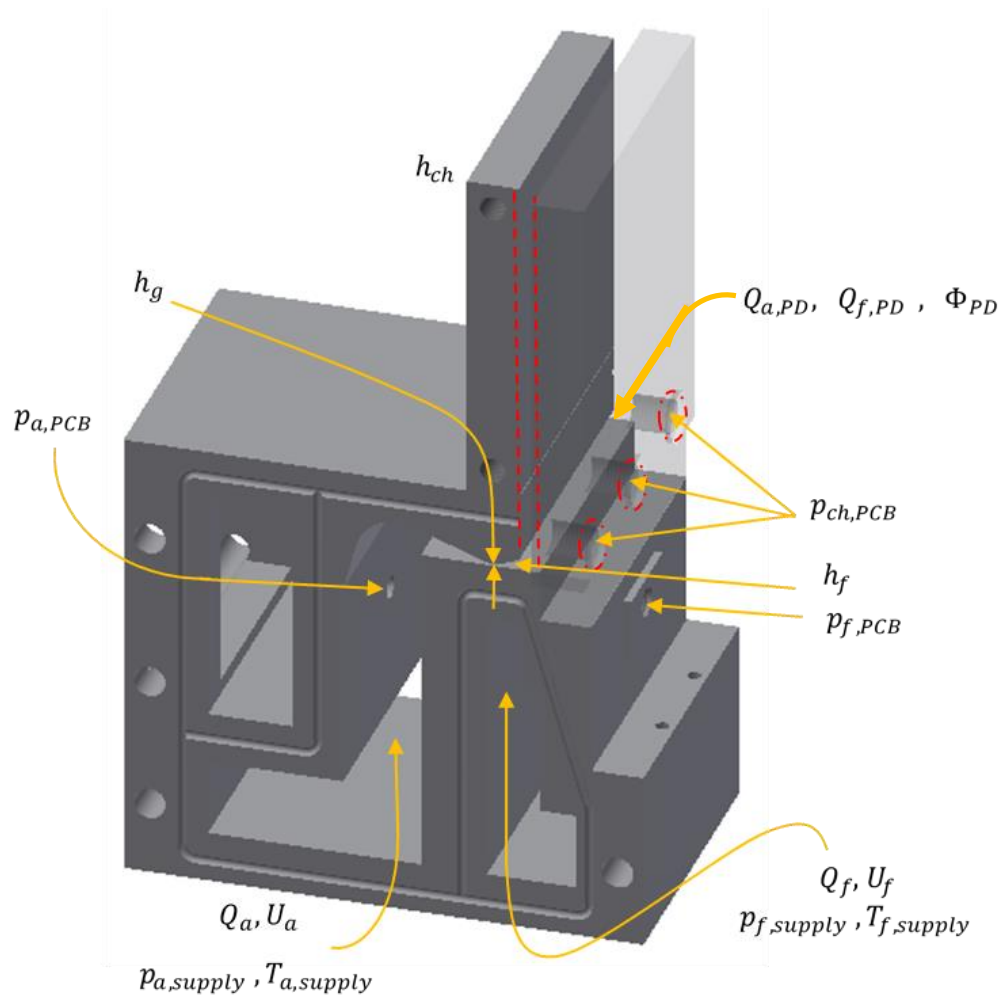

Figure 4.1: BSRDE Geometry and Setup

The dependent variable of interest here is the interruption time. In this case, the dependent variable is not physically represented in Figure 4.1. This is due to the nature of the parameter, which is 
derived through the temporal content of the pressure measurement to indicate the duration of time that the detonation event causes the pressure signal to rise above its steady state value. Independent variables are depicted with the equation below.

$$
\Delta t_{I N T}=f\left(p_{d e t}, h_{g}, Q_{a}, Q_{f}, p_{a, p l}, p_{f, p l}, U_{a}, U_{f}, \rho_{a}, \rho_{f}, h_{f}, w_{c h}, Q_{a, P D}, Q_{f, P D}, \tau\right)
$$

Thus, for Buckingham Pi, the r, k, and n values are given as:

$$
r=3 ; k=16 ; n=k-r=13 \text { ח terms }
$$

The $13 \Pi$ terms are worked out below, using the air supply density, air injection gap height, and air supply flow rate as repeating variables. These are selected due to their significance as testing conditions, measured values, or design considerations in this system.

$$
\begin{gathered}
\Pi_{1}=f_{1}\left(\Delta t_{I N T}, \rho_{a}, h_{g}, Q_{a}\right)=\frac{\Delta t_{I N T} Q_{a}}{h_{g}^{3}} \\
\Pi_{2}=f_{2}\left(p_{d e t}, \rho_{a}, h_{g}, Q_{a}\right)=\frac{p_{d e t} h_{g}^{4}}{\rho_{a} Q_{a}^{2}} \\
\Pi_{3}=f_{3}\left(Q_{f}, \rho_{a}, h_{g}, Q_{a}\right)=\frac{Q_{f}}{Q_{a}}=F A R_{a c t u a l, C H} \\
\Pi_{4}=f_{4}\left(p_{a, p l}, \rho_{a}, h_{g}, Q_{a}\right)=\frac{p_{a, p l} h_{g}^{4}}{\rho_{a} Q_{a}^{2}} \\
\Pi_{5}=f_{5}\left(p_{f, p l}, \rho_{a}, h_{g}, Q_{a}\right)=\frac{p_{f, p l} h_{g}^{4}}{\rho_{a} Q_{a}^{2}} \\
\Pi_{6}=f_{6}\left(U_{a}, \rho_{a}, h_{g}, Q_{a}\right)=\frac{U_{a} h_{g}^{2}}{Q_{a}} \\
\Pi_{7}=f_{7}\left(U_{f}, \rho_{a}, h_{g}, Q_{a}\right)=\frac{U_{f} h_{g}^{2}}{Q_{a}} \\
\Pi_{8}=f_{8}\left(\rho_{f}, \rho_{a}, h_{g}, Q_{a}\right)=\frac{\rho_{f}}{\rho_{a}}
\end{gathered}
$$




$$
\begin{gathered}
\Pi_{9}=f_{9}\left(h_{f}, \rho_{a}, h_{g}, Q_{a}\right)=\frac{h_{f}}{h_{g}} \\
\Pi_{10}=f_{10}\left(w_{c h}, \rho_{a}, h_{g}, Q_{a}\right)=\frac{w_{c h}}{h_{g}} \\
\Pi_{11}=f_{11}\left(Q_{a, P D}, \rho_{a}, h_{g}, Q_{a}\right)=\frac{Q_{a, P D}}{Q_{a}} \\
\Pi_{12}=f_{12}\left(Q_{f, P D}, \rho_{a}, h_{g}, Q_{a}\right)=\frac{Q_{f, P D}}{Q_{a}} \\
\Pi_{13}=f_{13}\left(\tau, \rho_{a}, h_{g}, Q_{a}\right)=\frac{\tau Q_{a}}{h_{g}^{3}}
\end{gathered}
$$

The first and last $\Pi$ terms can be combined to define the interruption time ratio. The flow rate and air gap height are cancelled out, and the term is left as a ratio of the interruption time and wave period. This parameter is mathematically represented by the time over which the pressure measurement is excited above the steady state value due to a detonation event divided by the period of the detonation wave. In other words, this term should give insight into the percentage of the period during which the detonation has influence over the injection system.

$$
\frac{\Pi_{1}}{\Pi_{13}}=\frac{\Delta t_{I N T} Q_{a}}{h_{g}^{3}} \cdot \frac{h_{g}^{3}}{\tau Q_{a}}=\frac{\Delta t_{I N T}}{\tau}
$$

The second $\Pi$ term is a peak channel pressure term made dimensionless by the air injection gap height, air supply density, and air supply flow rate. This term is ideal in that it combines a measured value with several designed and specified values, namely the gap height and flow rate. Mathematically, it can indicate the fluid dynamic pressure at the inlet plane due to the detonation compared to the momentum flux of air at the inlet. $\Pi_{2}$ will be investigated throughout this work and applied to both the BSRDE and LSRDE. It, along with the interruption time ratio, form the basis for analyzing relationships between the two rigs. 
Considering the stoichiometric fuel to air ratio, the third $\Pi$ term can be transformed into the equivalence ratio. This is the equivalence ratio based on the channel supply flow rates, which has no bearing on shock strength within the BSRDE, as will be shown later.

$$
\Pi_{3}=\frac{Q_{f}}{Q_{a}}=F A R_{\text {actual }, c h}=F A R_{\text {actual,ch }} *\left(\frac{1}{F A R_{\text {stoich }, c h}}\right)=\Phi_{c h}
$$

The ninth and tenth $\Pi$ terms can be transformed into area ratios. Similar ratios are used by Bykovski, et al. to represent the "expansion ratio" for the RDE [39].

$$
\begin{gathered}
\Pi_{9}=\frac{h_{f}}{h_{g}}=\left(\frac{N \pi h_{f}}{2 \pi r_{g}}\right)\left(\frac{h_{f}}{h_{g}}\right)=\frac{N \pi\left(\frac{h_{f}}{2}\right)^{2}}{2 \pi r_{g} h_{g}}=\frac{A_{f}}{A_{g}}=K_{f} \\
\Pi_{10}=\frac{w_{c h}}{h_{g}}=\left(\frac{r_{o}+r_{i}}{2 r_{g}}\right) \frac{w_{c h}}{h_{g}}=\left[\frac{\pi\left(r_{o}+r_{i}\right)\left(r_{o}-r_{i}\right)}{2 \pi r_{g} h_{g}}\right]=\left[\frac{\pi\left(r_{o}^{2}-r_{i}^{2}\right)}{A_{g}}\right]=\left[\frac{A_{c h}}{A_{g}}\right]=K_{c h}
\end{gathered}
$$

The eleventh and twelfth $\Pi$ terms can be combined to create an equivalence ratio within the predetonation tube. Here, the flow rates were chosen to create a desirable shock structure to be compared with the LSRDE. This value will be more applicable to the conditions seen in the LSRDE.

$$
\frac{\Pi_{12}}{\Pi_{11}}=\frac{Q_{f, P D} / Q_{a}}{Q_{a, P D} / Q_{a}}=\frac{Q_{f, P D}}{Q_{a, P D}}=F A R_{\text {actual }, P D} *\left(\frac{1}{F A R_{\text {stoich }, P D}}\right)=\Phi_{P D}
$$

Finally, the result is given below in Equation (4.12).

$$
\Delta t_{I N T} /_{\tau}=f\left(\frac{p_{d e t} h_{g}^{4}}{\rho_{a} Q_{a}^{2}}, \Phi_{c h}, \Phi_{P D}, K_{c h}, K_{f}, \frac{p_{a, p l} h_{g}^{4}}{\rho_{a} Q_{a}^{2}}, \frac{p_{f, p l} h_{g}^{4}}{\rho_{a} Q_{a}^{2}}, \frac{U_{a} h_{g}^{2}}{Q_{a}}, \frac{U_{f} h_{g}^{2}}{Q_{a}}, \frac{\rho_{f}}{\rho_{a}}\right)
$$




\subsubsection{BSRDE Detonation Characteristics Determination}

In order to obtain the dimensionless time and pressure variables introduced earlier, the interruption time and peak pressure must be determined. MATLAB code was developed to identify the location of peak signal during the detonation event, the arrival of the detonation event, and the dissipation of the detonation event relative to the sensor.

The location of peak signal was found by determining the location at which the signal breeches a certain threshold, then iteratively marching point by point until a set of constraints are satisfied. These constraints incorporate forward differences and the maximum slope in conjunction with the behavior of the sample points before and after each point within a specified window which is based on the sampling frequency. To decipher between actual detonation peaks and shock reflections, robustness was increased by analyzing combinations of forward differences in addition to adding time bounds based on the sampling rate and wave period.

Following peak finding, the routine works backward in time from the peak location to determine the detonation arrival point. This point is determined by iteratively comparing the slope between the point being analyzed and the previous five points. If the slope between the point and the previous five sample points are non-positive with time, then the detonation arrival conditions are satisfied as defined here. Finally, the time at which the detonation event defined as the time at which the pressure signal falls to $95 \%$ of the peak pressure compared to the minimum value between the peak and the next peak. In the BSRDE, there are significant influences from shock reflections, depicted in Figure 4.3 as the sharp dip in signal following the peak. Thus, the dissipation location is found by comparing a localized average signal value to the $95 \%$ constraint. Collectively, the detonation arrival, peak, and dissipation points will be referred to as the detonation characteristics from herein. 
A region of interest was determined by the calculated shock speed at the exit of the pre-detonator tube in accordance with previous work by Bedick, et al. [26]. It was used to focus on a useful domain of data, omitting large amounts of data that would slow analysis and yield no results. A representative case is shown below in Figure 4.2.

\section{Full Signal with Region of Interest}

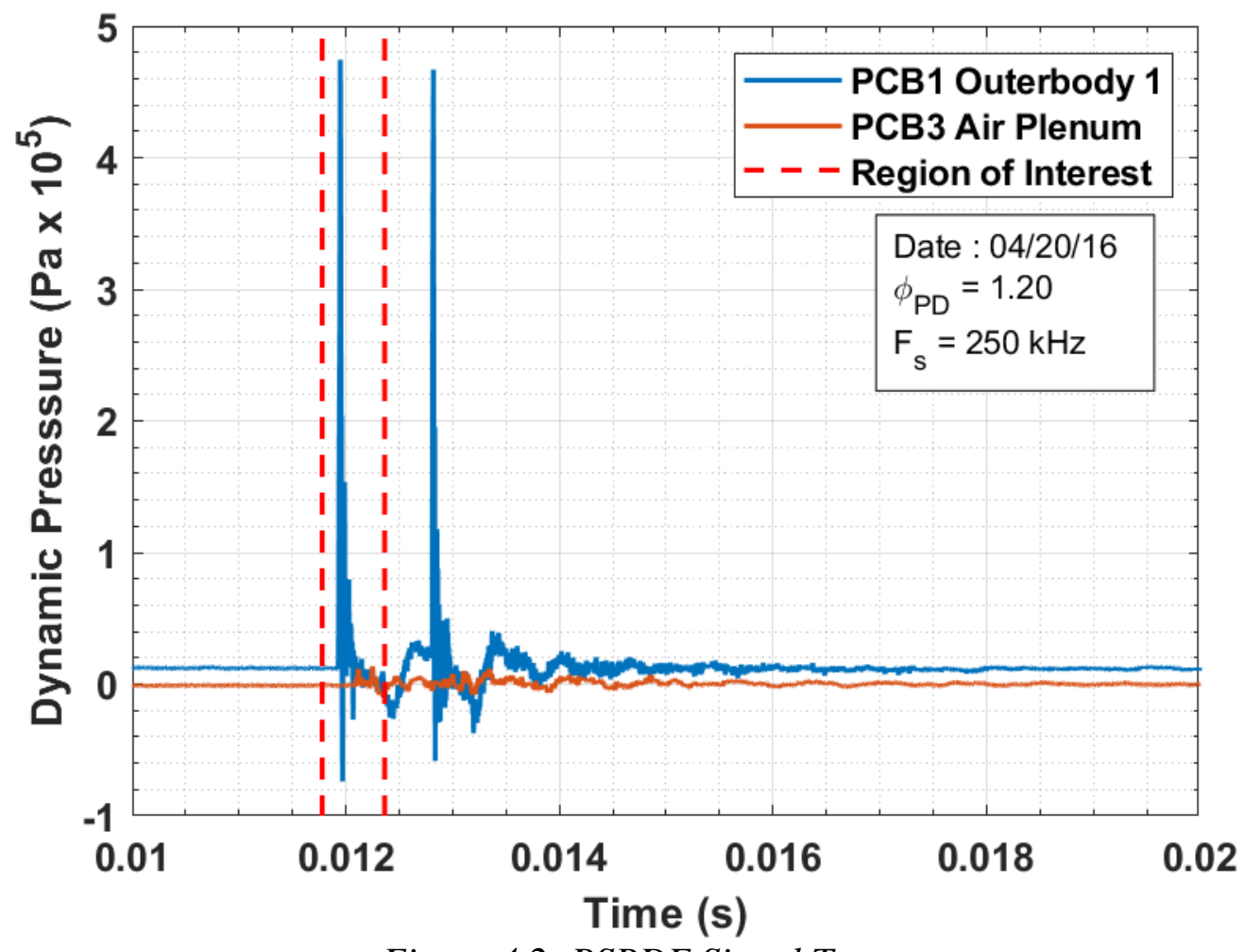

Figure 4.2: BSRDE Signal Trace

The two pressure peaks in the signal result from the split path tube indicated in Section 3.2. It was found that there was significant variability in the magnitude of the second peak relative to the first peak and to separate tests. For this reason, the second wave is omitted from this study. A representative BSRDE case is depicted in Figure 4.3, with time bounds on the figure being the region of interest defined in Figure 4.2. 


\section{Detonation Characteristics within Region of Interest}

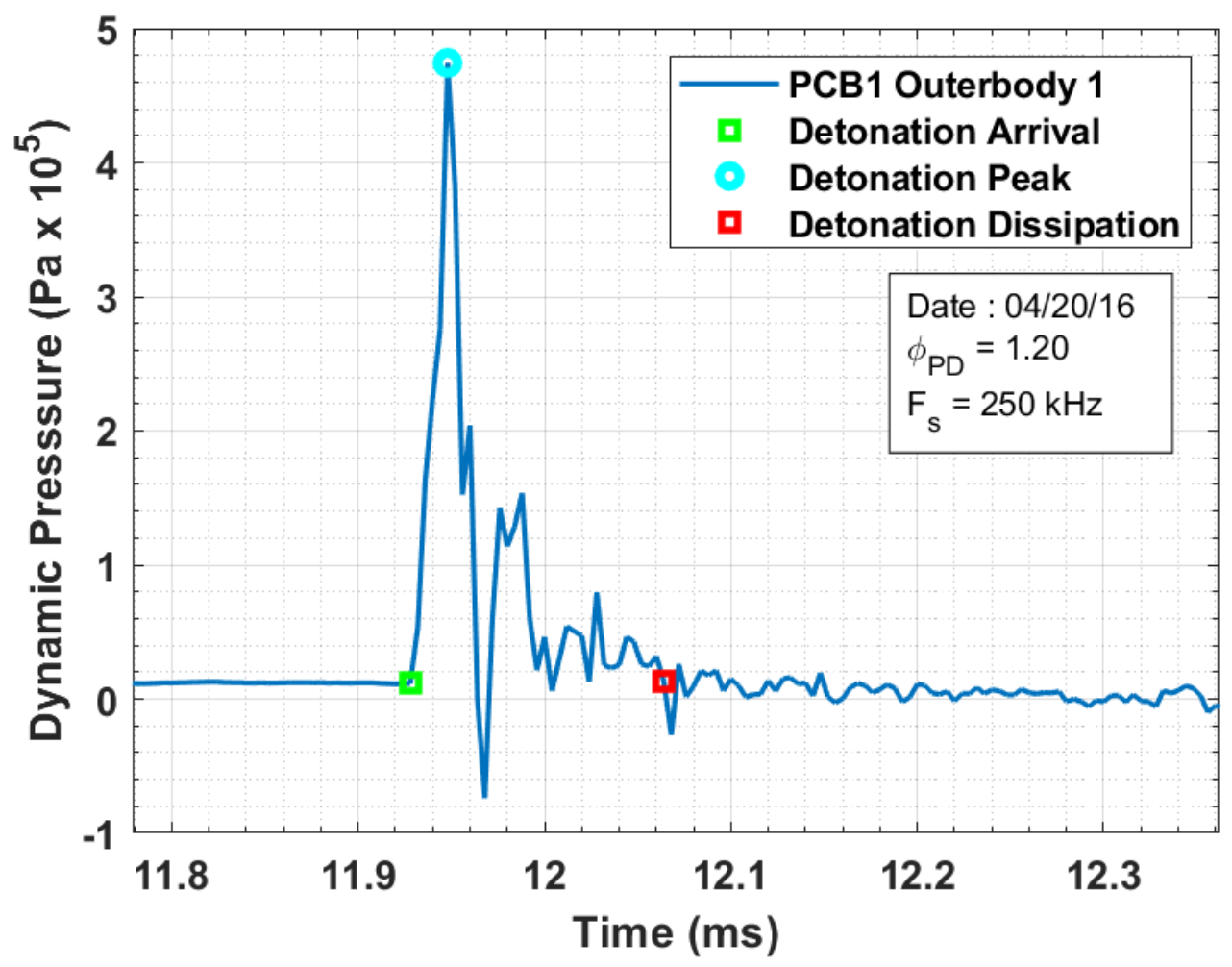

Figure 4.3: BSRDE Detonation Characteristics

These pressure signals can contain noise from various sources. It is important to investigate the presence of noise, generally, but especially in this study. Since the interruption time is directly dependent on the temporal content of the signal, it is imperative that every measure be taken to determine an accurate result.

One such source of noise is the resonance frequency of the measurement instrument itself. Manufacturers often list this parameter within the sensor specifications. A lowpass (LP) filter or band-stop filter can be applied to the raw signal to rid the signal of resonant frequency interference. Moving average filtering can also be applied to noisy signals. In this technique, several consecutive points are averaged and used to construct a smoother signal with a lower relative sampling rate. In either case, the filter is applied after the peak location to avoid attenuation of the peak value. The effects of the various filters are seen in Figure 4.4. 


\section{Various Filtering Techniques}

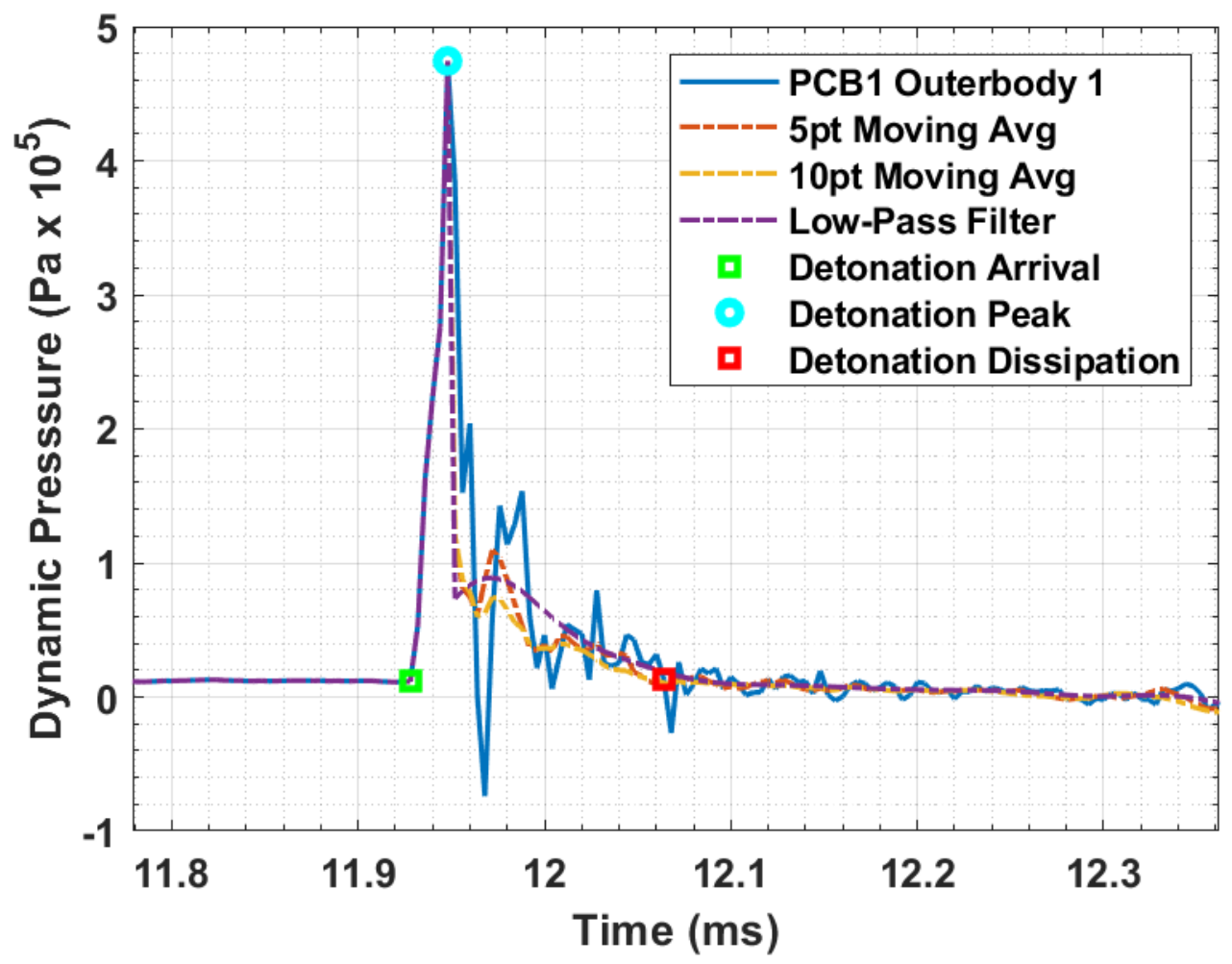

Figure 4.4: Filtering Techniques

The various filtering techniques were ultimately compared to the detonation arrival, peak, and dissipation locations. Each of these points were found using the raw signal and are compared to the filtered signals in Figure 4.4. Observe that the filtering has little to offer in this analysis due to the perceived accuracy of the detonation characteristic points. Therefore, filtering of the BSRDE data will be omitted. However, it was useful in ensuring that no significant sources of noise exist that may skew interruption time ratio results.

Recall that the goal in this data reduction was to create parameters which would be convenient to compare to the LSRDE. In acquiring the detonation arrival, peak signal, and detonation passing times, a detonation interruption ratio and dimensionless peak pressure value could be determined. 


\subsubsection{BSRDE Summarizing Data}

The individual detonation waves were plotted together to gain an understanding of repeatability. Each wave was aligned by the peak signal time and organized by air supply flow rate. The 700 SLPM case is provided in Figure 4.5. Though the peak pressures range significantly, the width of the detonation signal is not varied greatly.

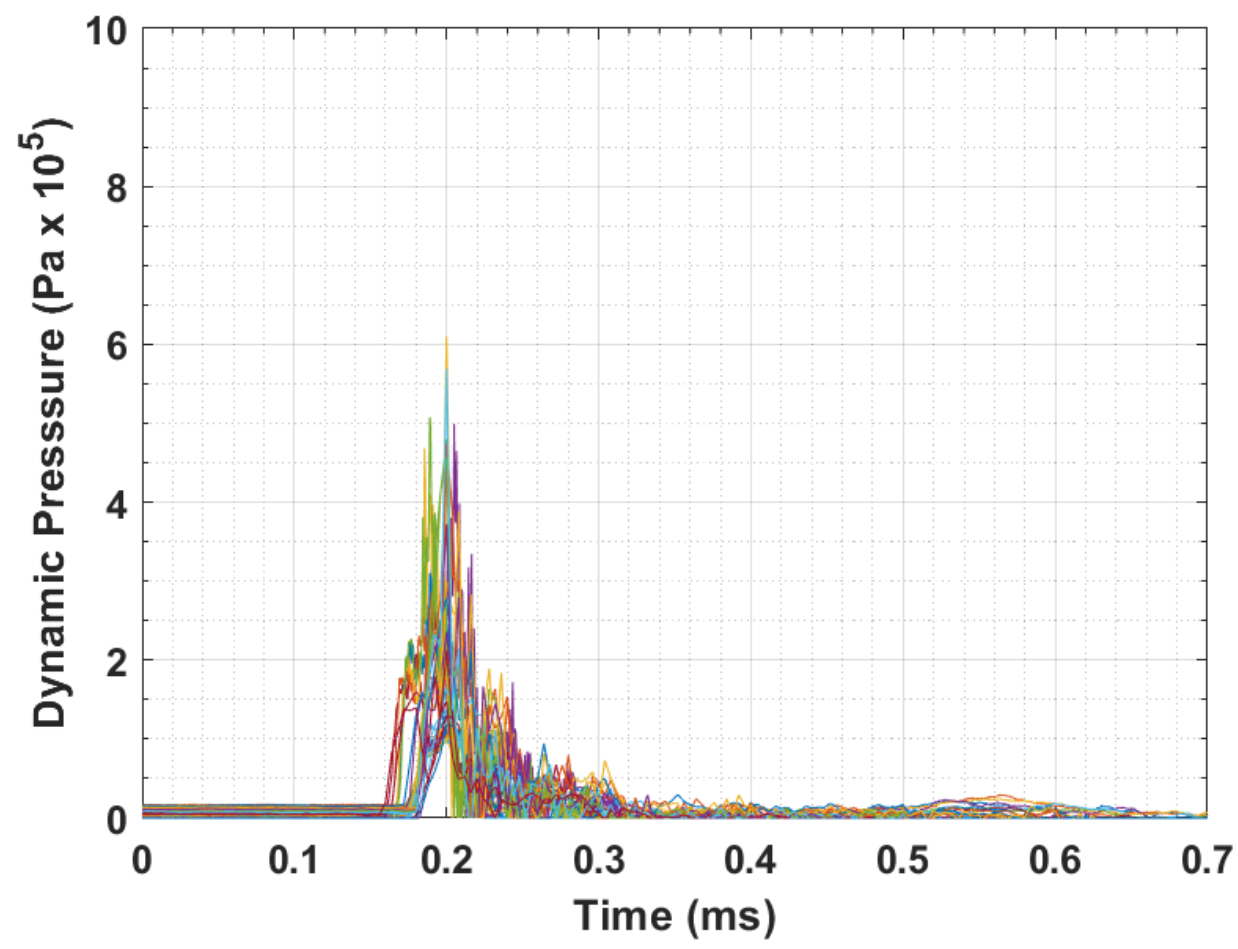

Figure 4.5: BSRDE 700 SLPM wave stacking

If we simply plot the channel pressure, non-dimensionalised by the plenum pressure, versus the interruption time, Figure 4.6 is produced. The data appear to lack any consistent trend. Within the 500 SLPM data set, there is an exponential trend with asymptotic behavior vertically at an interruption time of $0.1 \mathrm{~s}$ and horizontally at $P_{c h}=2 \times 10^{5} \mathrm{~Pa}$. The vertical asymptote could be indicative of a lower limit on the interruption time. In other words, it would suggest that there is some upper bound on system operating frequency at which point sustained operation is not possible. The horizontal asymptote is interesting in that it promotes a sharp rise in interruption 
time with decreasing peak pressure. These trends, however, are not strong and are not supported by the additional flow rate cases.

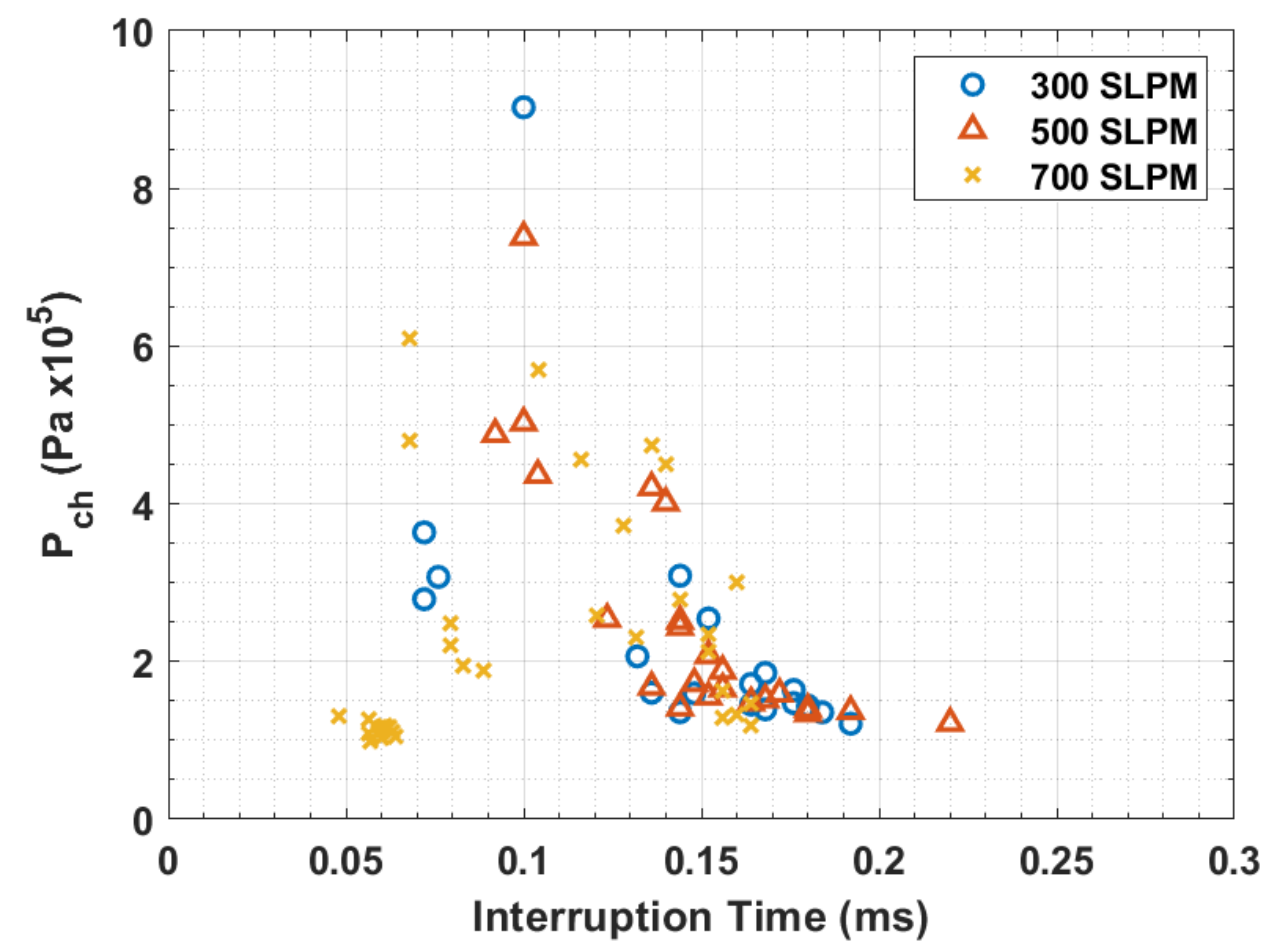

Figure 4.6: Effect of Air Supply Flow Rate on Pressure ratio and interruption time

Prior to calculating the second $\Pi$ term as determined in the dimensional analysis, each component of the term was scrutinized to gain insight into the influences each term has on the parameter. In performing a dimensional analysis, isolation of key parameters is the goal. By checking the variation in each of the contributing variables, much is learned about $\Pi_{2}$.

For the BSRDE, the air injection height and peak signal should not change, and the air flow rates are controlled. Additionally, one singular value for the RDE period is used, which is based on the overall dominant frequency across the entire test obtained via a frequency analysis. Thus, the $\Pi_{2}$ term plotted against interruption ratio should look identical to the peak signals plotted against interruption time. In Figure 4.7, the air supply density and peak pressure variations are plotted against air supply flow setpoint. 


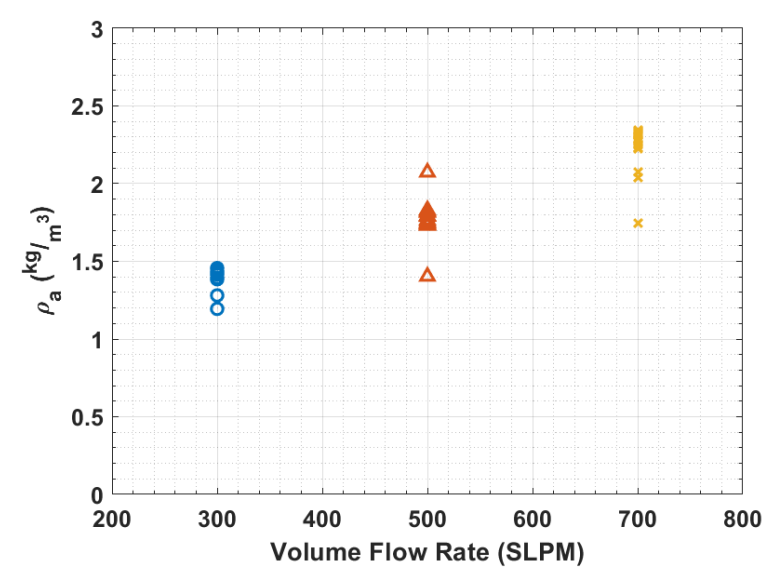

(a) Density variation

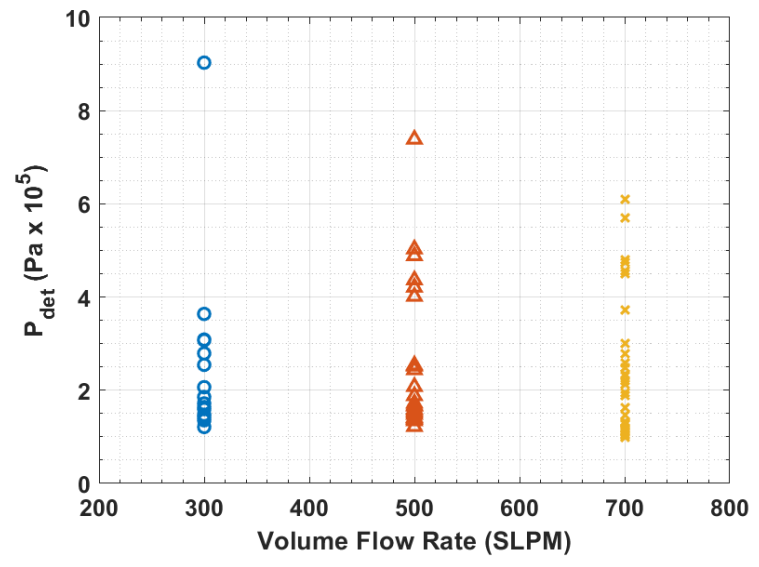

(b) Pressure variation

Figure 4.7: Variation in the parameters which make up the $\Pi_{2}$ term

Figure 4.7(a) clearly indicates that there is some variation in the air supply density. Upon further investigation it was found that this is due directly to the increased flow rate. As the air supply flow rate increases, the air supply static pressure increases which was used to determine the density via the ideal gas law.

In Figure 4.7(b) it is observed that there is little variation in detonation peak pressure with changing air supply flow rate. This is due to the experimental design and the mechanism by which the shock is derived. In the BSRDE, the pre-detonator tube air supply, fuel supply, and equivalence ratio dictate the shock strength. A design consideration was to generate a repeatable shock strength to facilitate learnings of inlet response due to other variables. Essentially, shock strength in this facility is designed to be decoupled from the plenum supply flow rates, indicated by the consistent range across each flow condition. While no consistent variation in peak pressure exists with varying flow rate, there is substantial variation within each flow rate condition. Within each set of data, the peak pressure varies by at least $250 \mathrm{kPa}$.

Having analyzed the parameters contributing to the dimensionless pressure term, the $\Pi_{2}$ term can be assembled and plotted, as shown in Figure 4.8. 


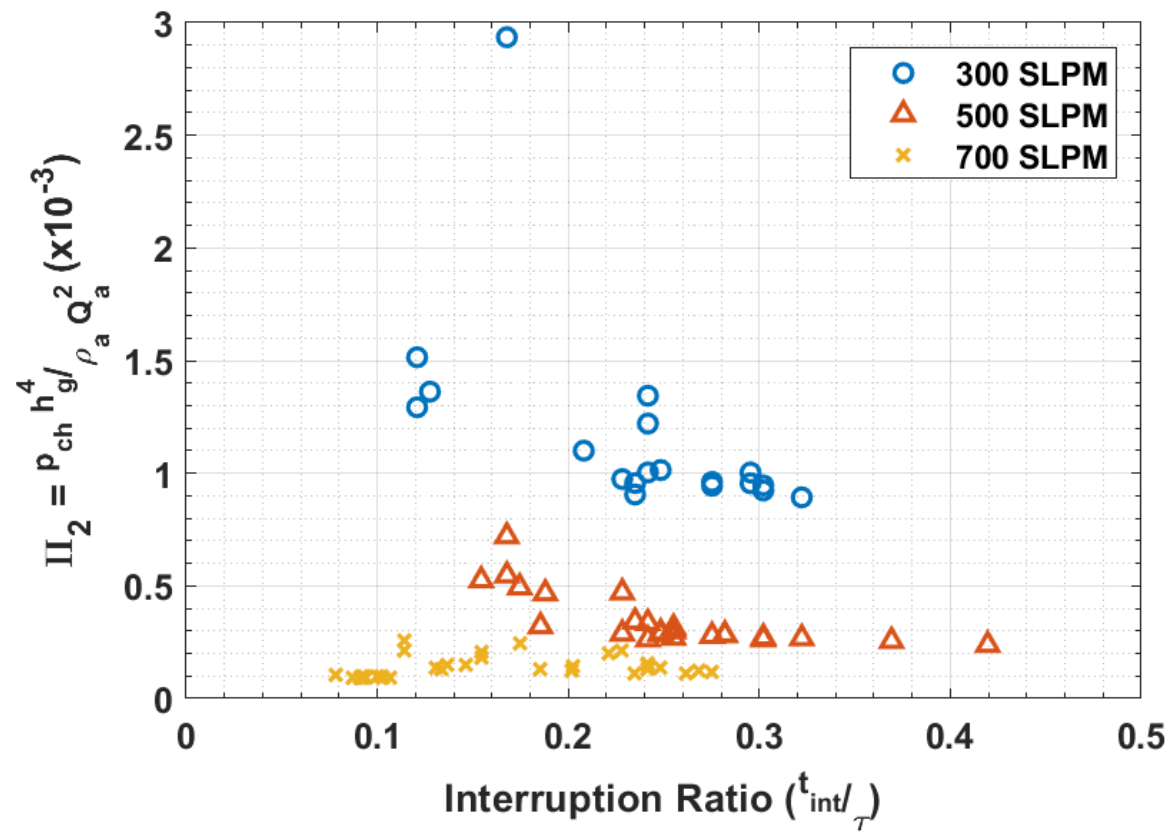

Figure 4.8: $\Pi_{2}$ versus interruption time ratio for varying air supply flow rates

The non-dimensionalization proves to better organize the data relative to Figure 4.7. Figure 4.8 does suggest trends and relationships between the two variables. These patterns will be further discussed in Section 6.1. Table 4.2 shows the BSRDE data analyzed in this study. This data set allows for a comparison of various supply air flow rates on interruption time ratio and dimensionless pressure.

Table 4.2: BSRDE data matrix (Number of Data Sets for Each Set of Test Conditions)

\begin{tabular}{|c|c|c|}
\hline $\mathbf{Q}=\mathbf{3 0 0}$ SLPM & $\mathbf{Q}=\mathbf{5 0 0}$ SLPM & $\mathbf{Q = 7 0 0 ~ S L P M ~}$ \\
\hline 19 & 24 & 35 \\
\hline
\end{tabular}

\subsection{LSRDE Methodology}

Analysis for the LSRDE was similar to that of the BSRDE above. The main difference was that in the LSRDE, detonation waves are seen continuously through the duration of the run. With such a high cycling frequency of $\sim 5000 \mathrm{~Hz}$ and a test duration of 6 seconds, there are potentially $\sim 30,000$ waves seen in each data set. To accommodate for this, a stable region is first sought out. Stability 
determination will be discussed first, followed by the detonation characteristics reduction and summarizing figures.

\subsubsection{Stability and Domain Selection}

Determining a time domain in which system stability is maximized is of utmost importance while investigating new analysis techniques. This process involves employing various filtering techniques and types to manipulate the data and speculate on its stability. Figure 4.9 gives a representative raw signal from the PCB transducer. The low frequency drift of the signal is due to the influence of heat transfer to the transducer.

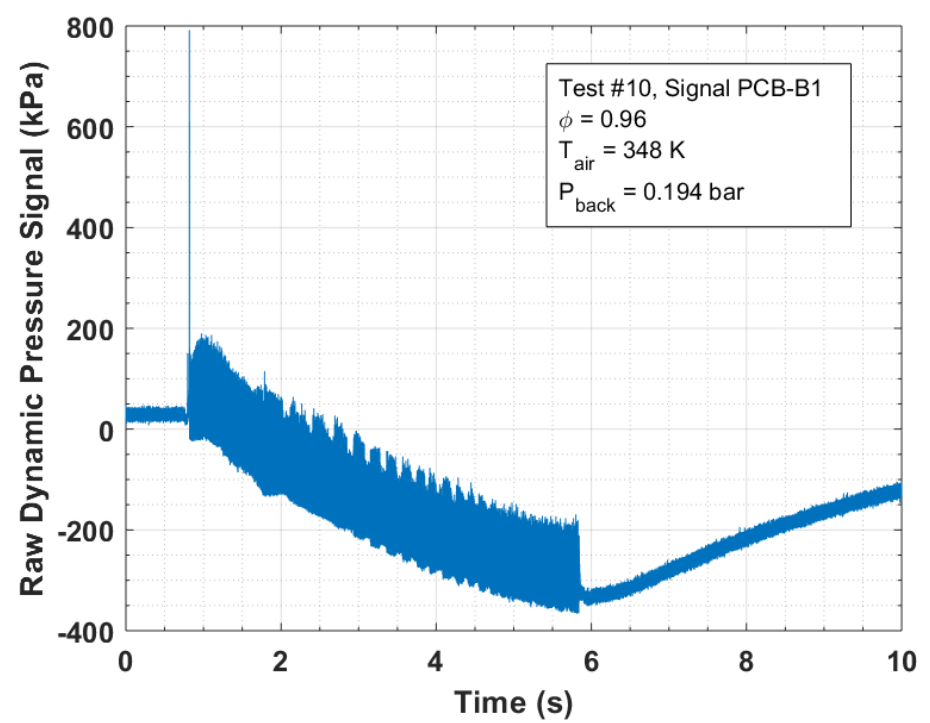

Figure 4.9: Raw signal for a representative case

This introduces the need to filter out temperature drift in the raw data due to heat transfer into the sensor. Temperature drift filtering is accomplished by employing a Butterworth filter, which can be used in MATLAB by calling the 'butter' command. Figure 4.10 provides a five second section of data which begins at the point of ignition. In Figure 4.10(a), the raw signal is displayed. Figure 4.10(b) demonstrates the impact of the Butterworth filter for dealing with temperature drift. 


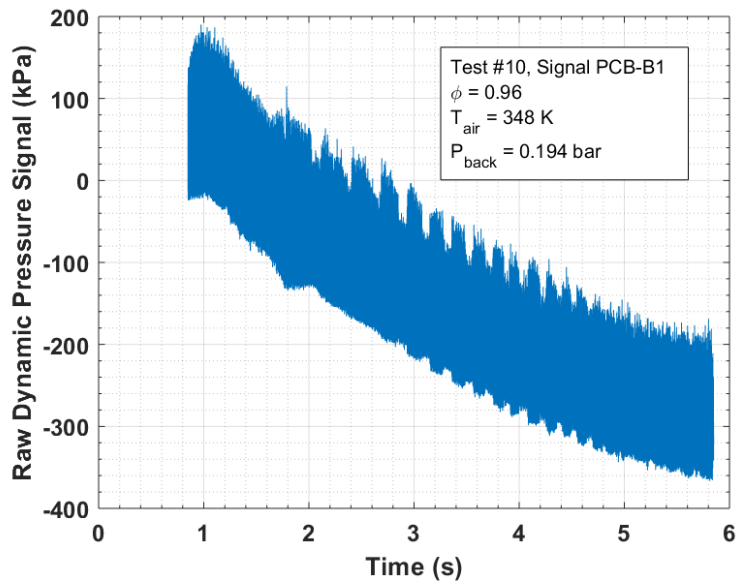

(a) Raw data before Butterworth filter

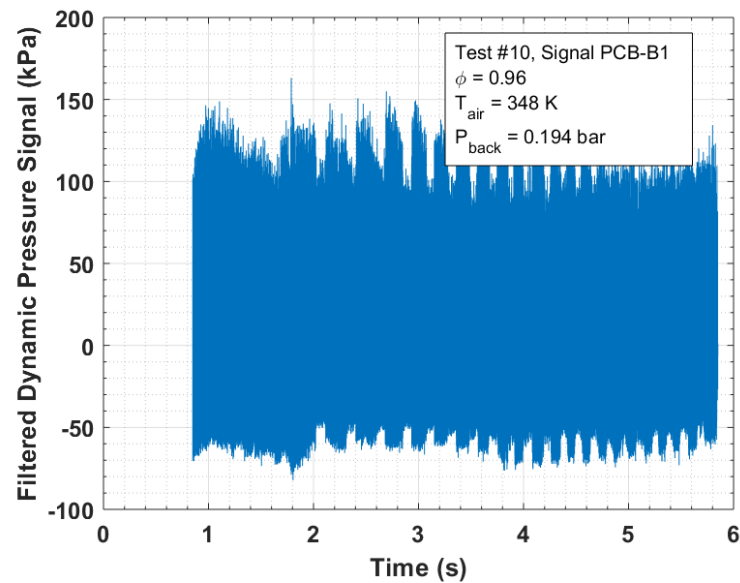

(b) Raw data after Butterworth filter

Figure 4.10: Effect of Butterworth filtering for temperature drift

To accommodate for the massive number of wave-passes within each data set, the MATLAB routine narrows down the data to be analyzed by utilizing a full-width at half-maximum (FWHM) evaluation. The full-width at half-maximum has many applications and is generally used to describe the extent of a function given by the difference of the independent variable for two points at which the dependent variable is at half of it's maximum value. For a sine wave, the FWHM becomes the wave length. In this case, it is used to suggest stability within the RDE system, thus is employed on the frequency content of the signal rather than the signal itself. An estimate for the power spectral density (PSD) is found by using Welch's estimate on the pressure signal. The tallest peak from the resulting PSD represents the frequency obtaining the most energy within the signal and is selected to be evaluated for FWHM. The width of the peak at half of it's magnitude is recorded in Hertz. These FWHM values are tracked for each window of data. The window width was specified to be 1/32 seconds. This process is depicted below in Figure 4.11. 


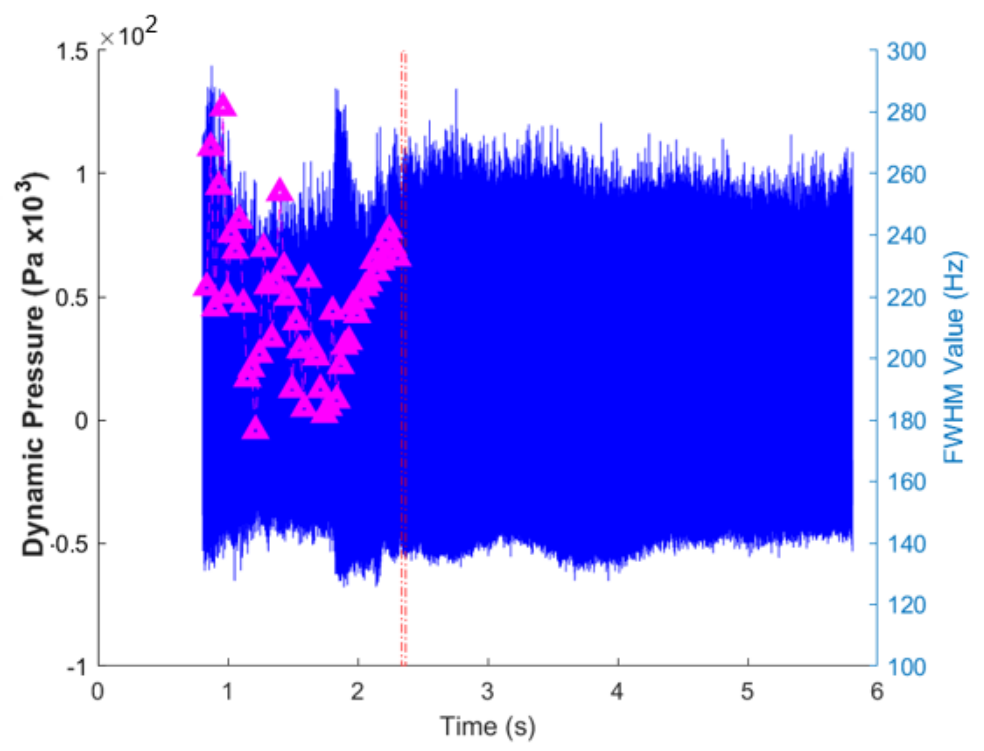

(a) Raw data (after Butterworth filter) showing window selection for FWHM calculation

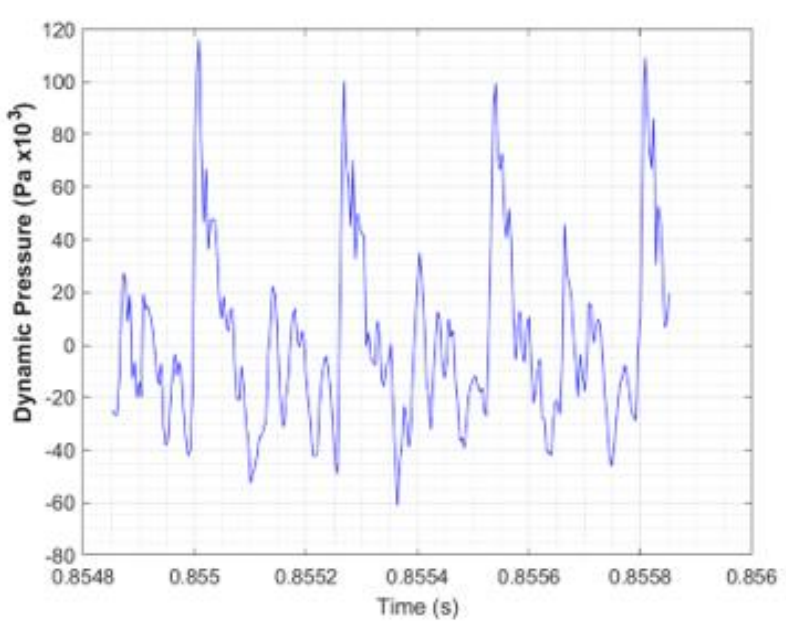

(b) Section of data selected

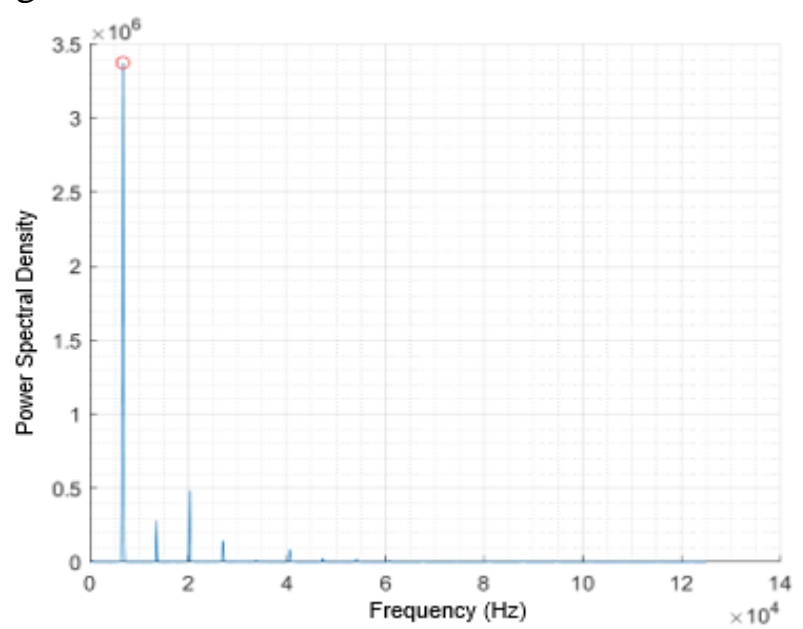

(c) FFT of (b)

Figure 4.11: Process of FWHM stability determination

The window sizing is somewhat arbitrary. An analysis of the impact of the window width on the resulting FWHM values was performed, with little conclusion. Figure 4.12 does depict some correlation from one to the next, however, the magnitudes change significantly, which is to be expected. It is logical that as the time interval for each window shrinks, the variation and instability within the local PSD increases. It is seen that the troughs in the 1/32 second plot and the remaining plots line up. This was deemed to be sufficient for this work, which does not attempt to specifically investigate system stability over time. This analysis was simply used to select a reasonable amount 
of data to analyze. The periodicity in the FWHM was thought to be an indicator of some underlying noise, however upon further investigation, it was not consistently repeatable across various test cases.

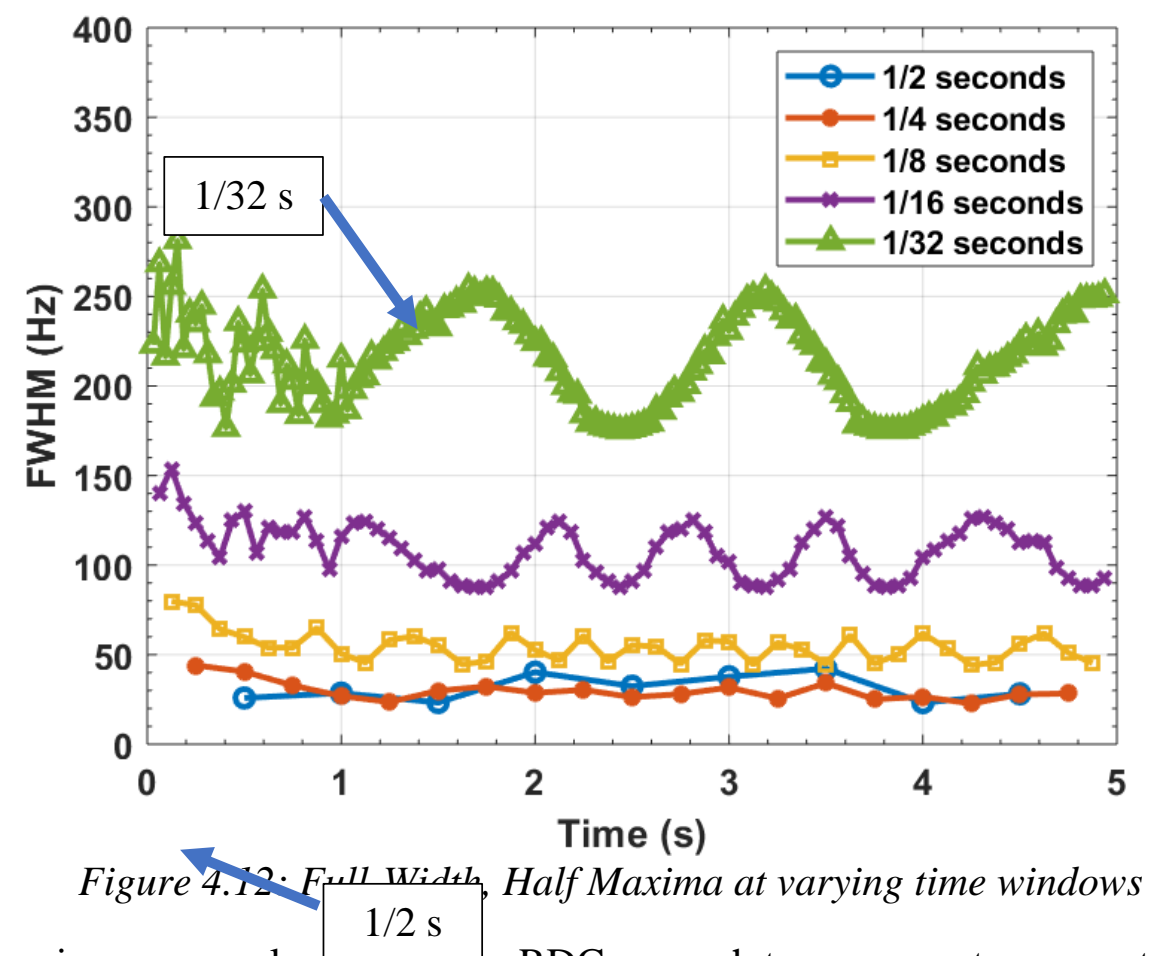

A spectrogram is a commonly usecu coon $\mathrm{RDC}$ research to assess system operational modes and relative stability. The color contours in a spectrogram represent the amplitude of the spectral energy in a particular frequency band at a given time. Essentially, each vertical strip is a 1D representation of Figure 4.11(c) where the red contour indicates peak and blue indicates minimum. Thus, the sharp red lines in Figure 4.13 indicate the highest amplitude of spectral energy and can be used to indicate cycling frequency of the detonation wave within the RDC. Any blending of colors in the spectrogram can indicate instabilities or unsteadiness. In other words, a lack of concentration and sharp color contrast in the spectrogram suggests that the system does not have a dominant operating frequency. The black dotted lines tracing horizontally across the spectrogram indicate the operating frequency based on one, two, and three-wave modes, increasing in frequency 
as the number of waves increases. These frequencies are determined by applying CJ theory, discussed in Section 1.7, under the system testing conditions. This is useful in determining the number of waves cycling in the RDC detonation channel at any instant in the time domain. Recall from CJ theory, the theoretical CJ velocity will never be reached due to non-idealities and losses. Thus, in this case, the system is operating in a two-wave mode due to the fact that the most intense red line, and therefore frequency of the highest spectral energy amplitude, exists below the second black dotted line working upward from $0 \mathrm{kHz}$. The 1/32 seconds FWHM values are then plotted on top of the spectrogram to compare the stability seen in the FWHM to that within the spectrogram.

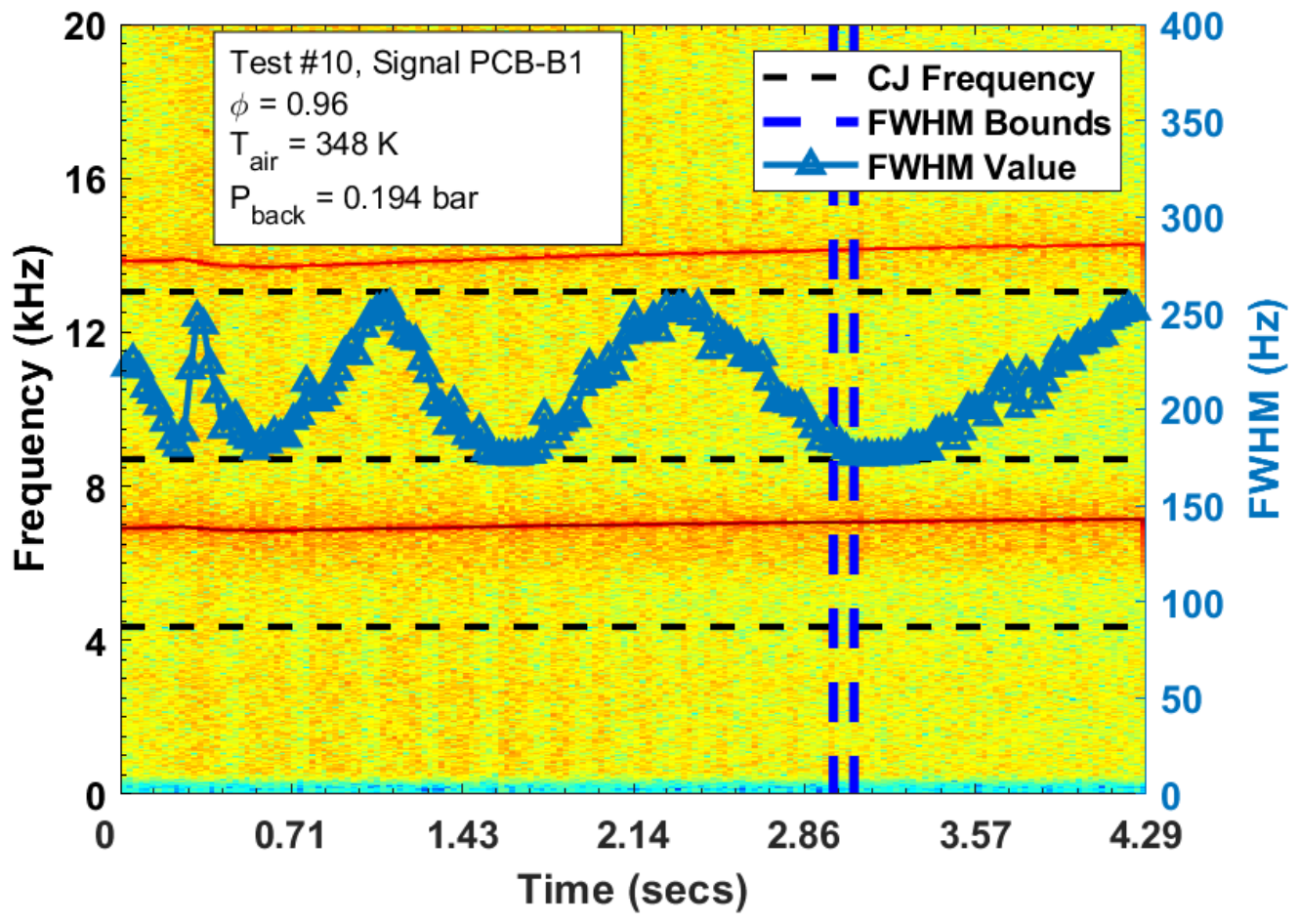

Figure 4.13: Spectrogram and FWHM for a representative case

It is observed in this case that the system is stable and steady, indicated by the flatness of the red contour at $\sim 6.5 \mathrm{kHz}$, and the contrast in color contours directly above and below the concentrated red line. 


\subsubsection{LSRDE Detonation Characteristics Determination}

Within the region of lowest FWHM values, the routine finds the shape of each detonation wave by determining the peak, arrival, and dissipation time for each wave passing. This routine is similar to that used in the BSRDE, however, more robust as the RDE necessitates finding these data for thousands of waves. A sample of the domain analyzed in this way is presented in Figure 4.14.

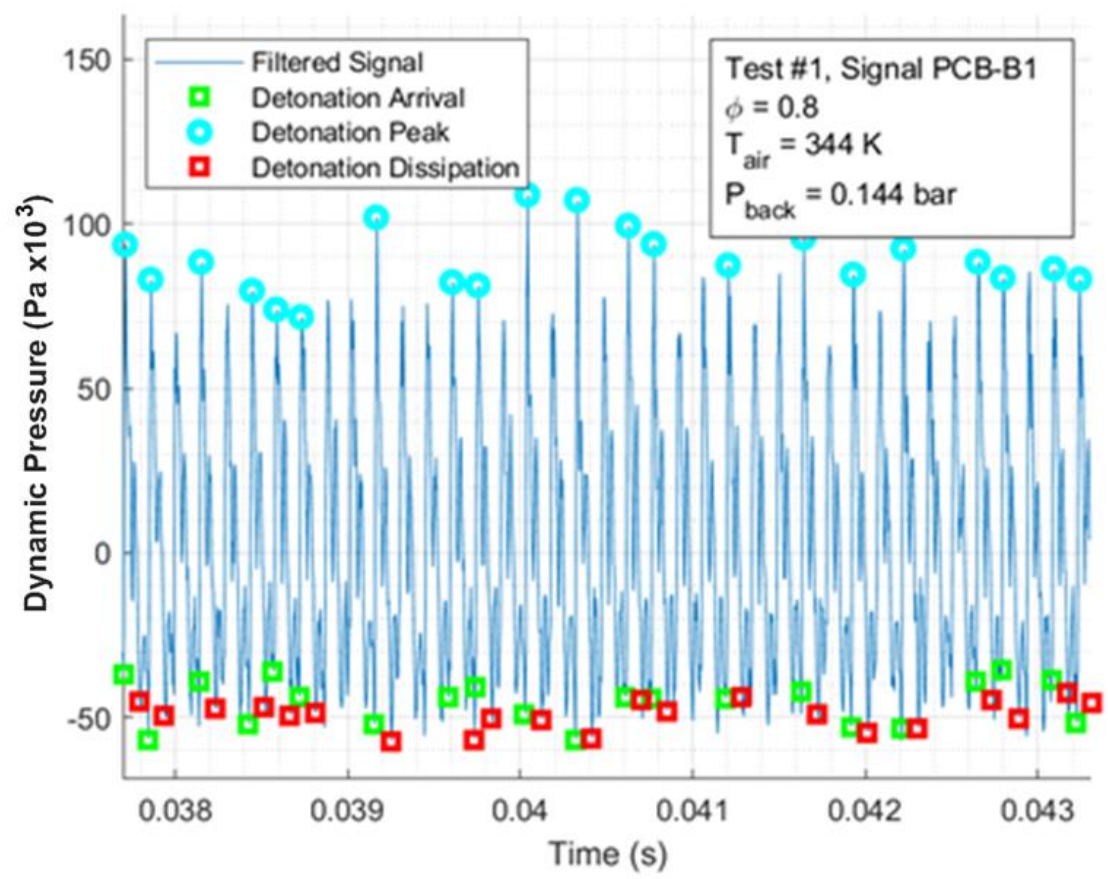

Figure 4.14: Detonation characteristics for a representative case

Note that some peaks are missed. This is due to variation in the system and difficulty clearly differentiating which points are detonation peaks and which are reflections or sources of noise. This is permittable as the MATLAB routine does not consistently discriminate a specific amplitude or interruption time.

Using these data, the routine cuts out each wave from the signal and aligns them by their peaks. All of the waves within this region of data can then be plotted together, in Figure 4.14, to show repeatability. This process generally produced surprisingly agreeable results. In Section 6.2, the 
results for each set of testing conditions will be presented. These plots were revealing of RDC operation as they visually capture the variability within the system due to the various testing conditions.

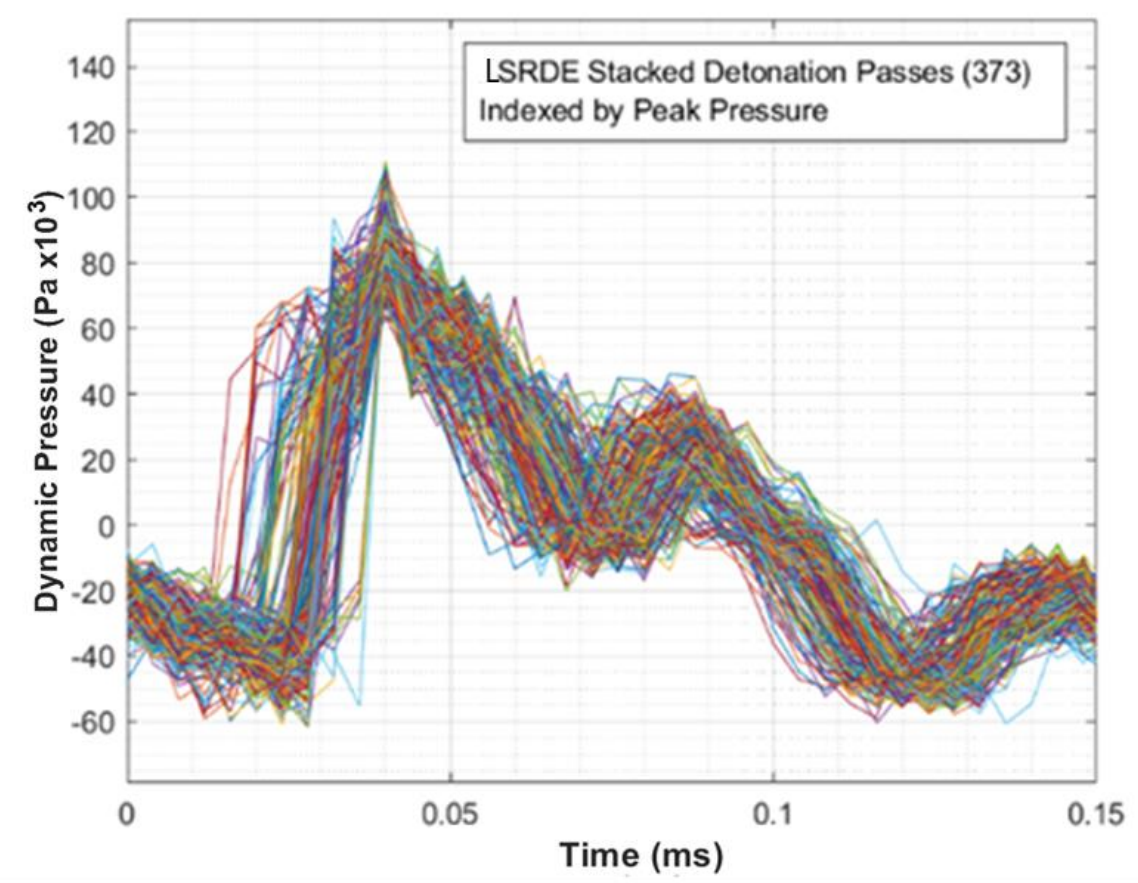

Figure 4.15: LSRDE wave stacking

Probability distribution functions were also calculated for the peak signals and interruption times. It is seen in Figure 4.16 that the peak pressures closely follow a normal distribution. This is likely due to the nature of acquiring these values. Based on the statistical definition, the sampling of the peak signal is a random process. It follows that the peak signals should be distributed about the mean, which is seen experimentally here.

The interruption time is a reduced value that is subject to bias due to the methods of determination in addition to discretization errors. Since it is not a directly measured or calculated value, there is surely some inherent uncertainty in the way it is calculated and therefore the PDF of interruption time is omitted here as it simply demonstrates that the interruption time does not follow a normal distribution. One such implication of uncertainty in the interruption time calculation is the 
sampling frequency. Since the frequency of operation of this particular data set is roughly $6.5 \mathrm{kHz}$ (observed from Figure 4.13), that leaves only 35 samples within each period, thus the resolution of the interruption time is low.

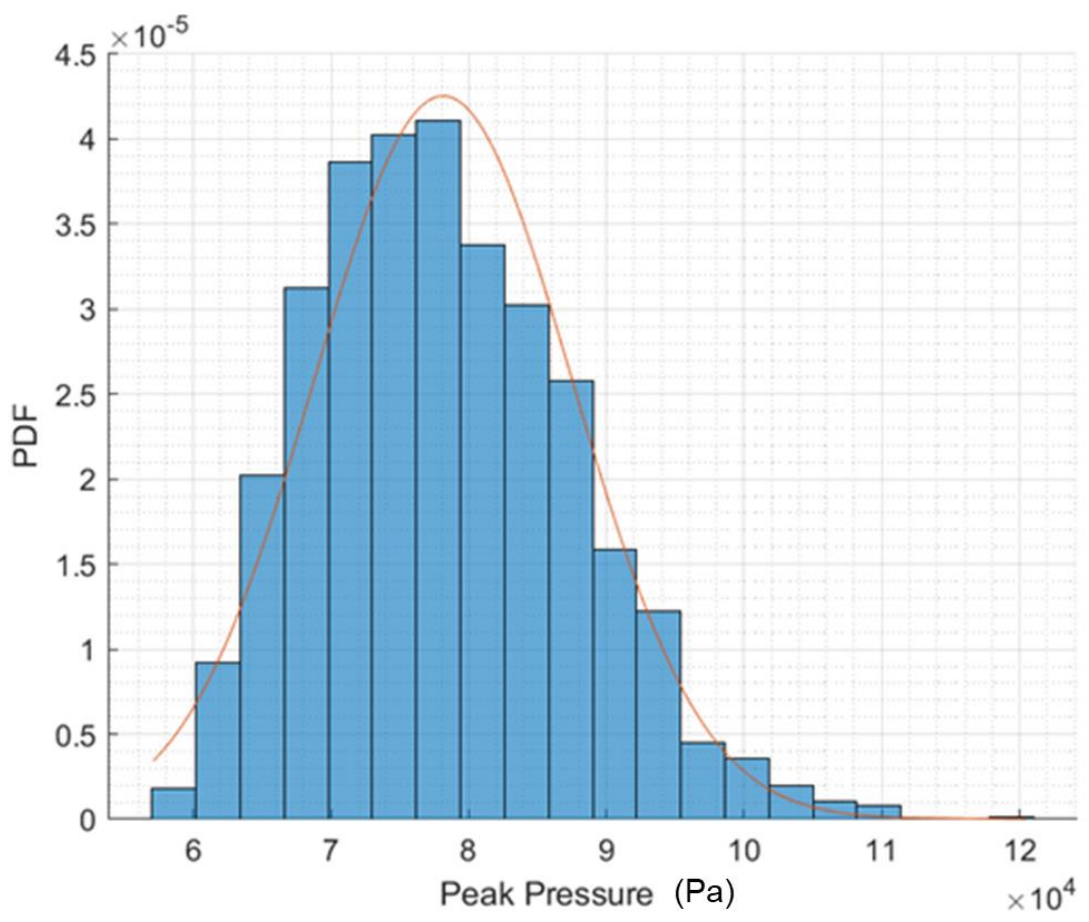

Figure 4.16: Probability distribution of peak pressures

This close resemblance of a normal distribution is desired because it allows for proven statistical analyses to be applied to the system. Applications include considering outliers based on Chauvenet's Criterion and analyzing uncertainty of the system. Both applications will be discussed in further detail in later in this section and in Section 5, respectively.

The reduced set of peak detonation pressures and interruption time ratios can then be plotted. The result is given below in Figure 4.17. 


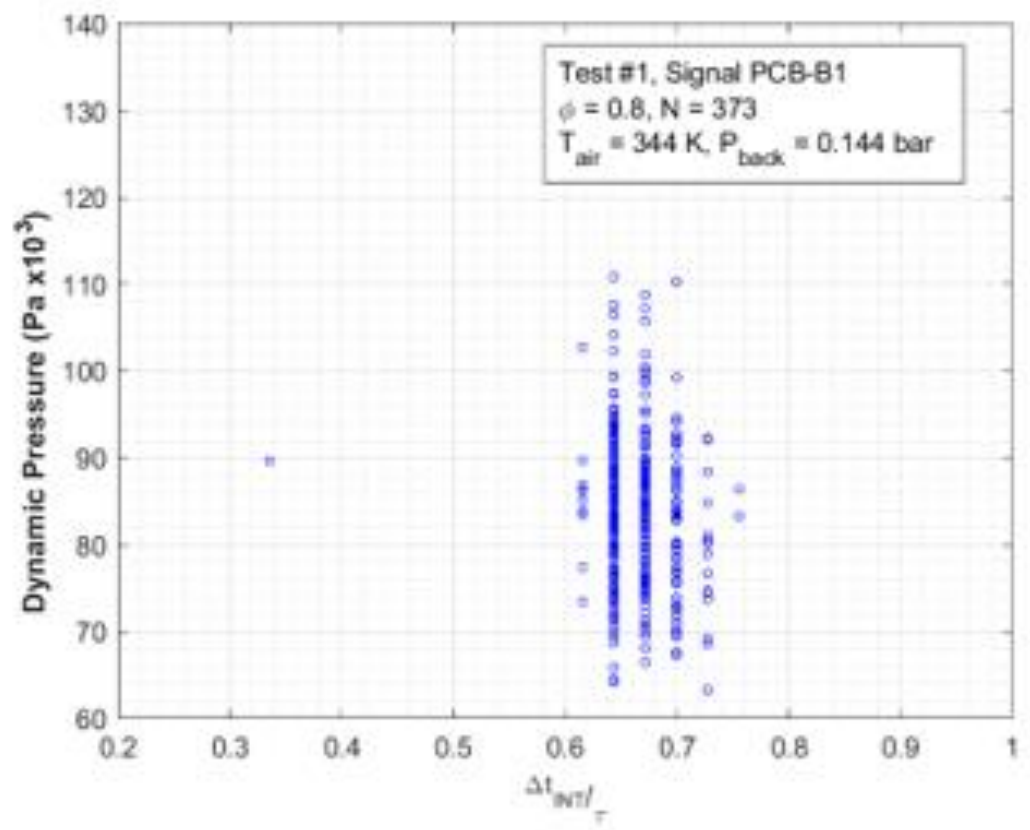

Figure 4.17: Distribution of pressure peaks and interruption time ratio

Similar to the results in the BSRDE, the dimensionless $\Pi_{2}$ parameter is plotted against interruption time ratio. In this case, there are thousands of data points per testing condition. Figure 4.18 depicts just one case. Each wave within the selected domain is represented by one point.

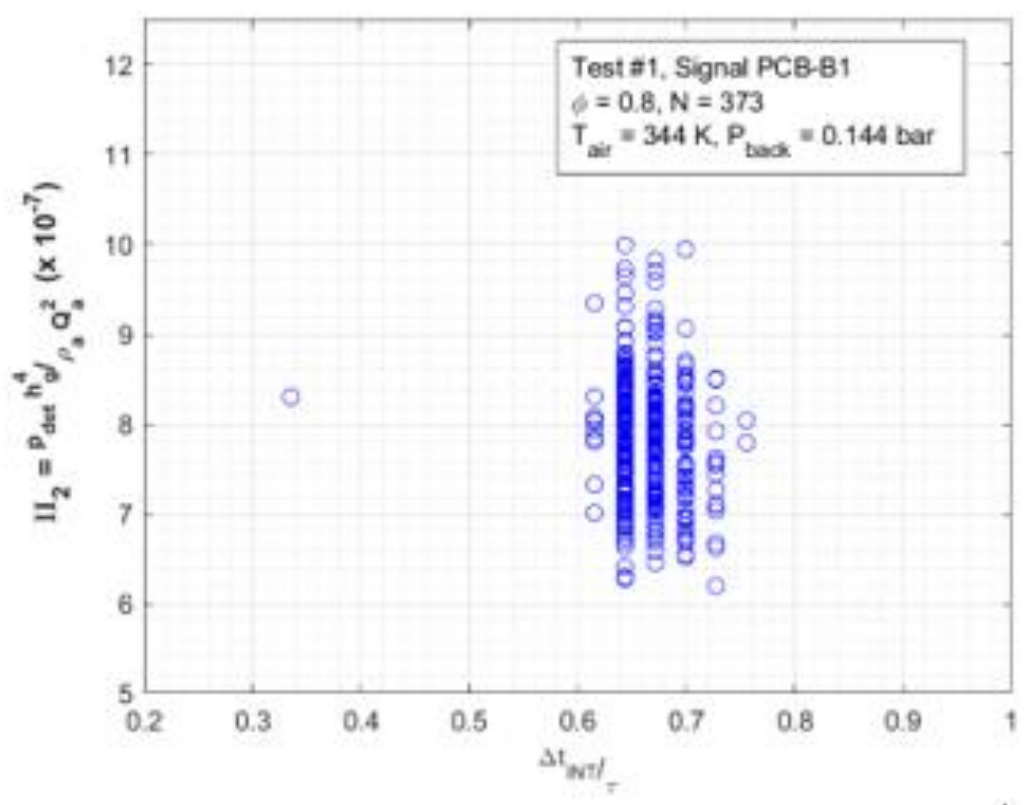

Figure 4.18: $\Pi_{2}$ versus interruption time ratio for a representative case 
The discretization seen in Figure 4.18, in the form of distinct horizontal groups of data, is due to the sampling frequency. Currently, the LSRDE instrumentation is sampled at $250 \mathrm{kHz}$. This value should be increased in order to reduce the discretization error, and uncertainty, in the determination of the interruption time. In the non-dimensionalised pressure versus interruption time ratio plots, there is an obvious presence of discretization. To further investigate this issue, the interruption times for each wave were plotted against the duration of the test.

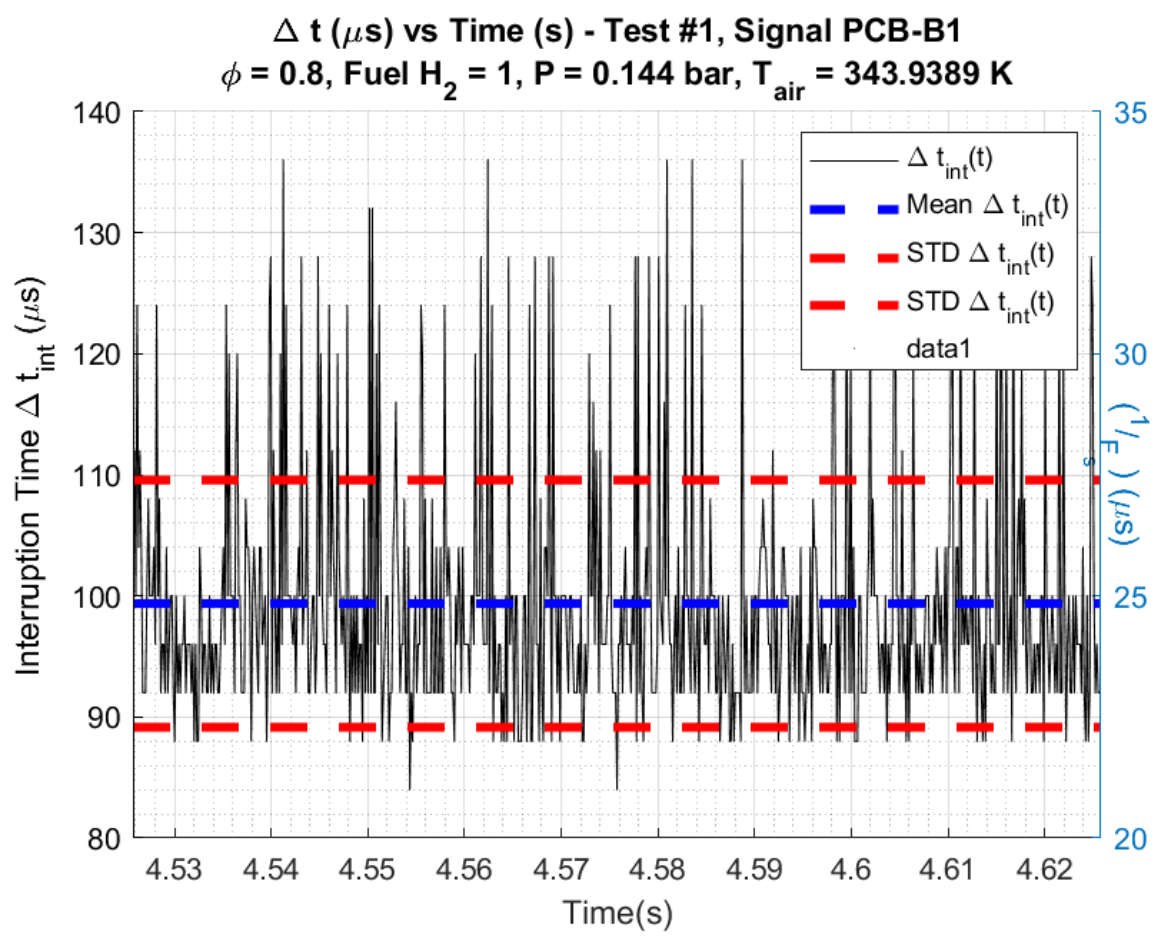

Figure 4.19: Interruption time versus time, showing discretized results due to low sample rate

The right y-axis shows multiples of the reciprocal of the sampling rate which is equivalently stated as number of samples. As seen in the Figure 4.19, the discretized levels that the interruption time are organized into line up with an integer multiple of the time-step. As mentioned previously, in a system operating at $6 \mathrm{kHz}$ and sampled at $250 \mathrm{kHz}$, only 35 samples exist within the RDE period. Considering that the interruption time ratio is consistently above $50 \%$ of the RDE period, this only allows for 17 distinct solutions for interruption time. A further 
consequence of this is that the uncertainty due to the sampling rate is $\pm 8 \mu \mathrm{s}$, which turns out to be $\sim 6 \%$ of the wave period based on an FFT analysis.

To eliminate visual influences in the data due to the sampling error, a contour plot of Figure 4.18 is generated. A representative case is presented in Figure 4.20.

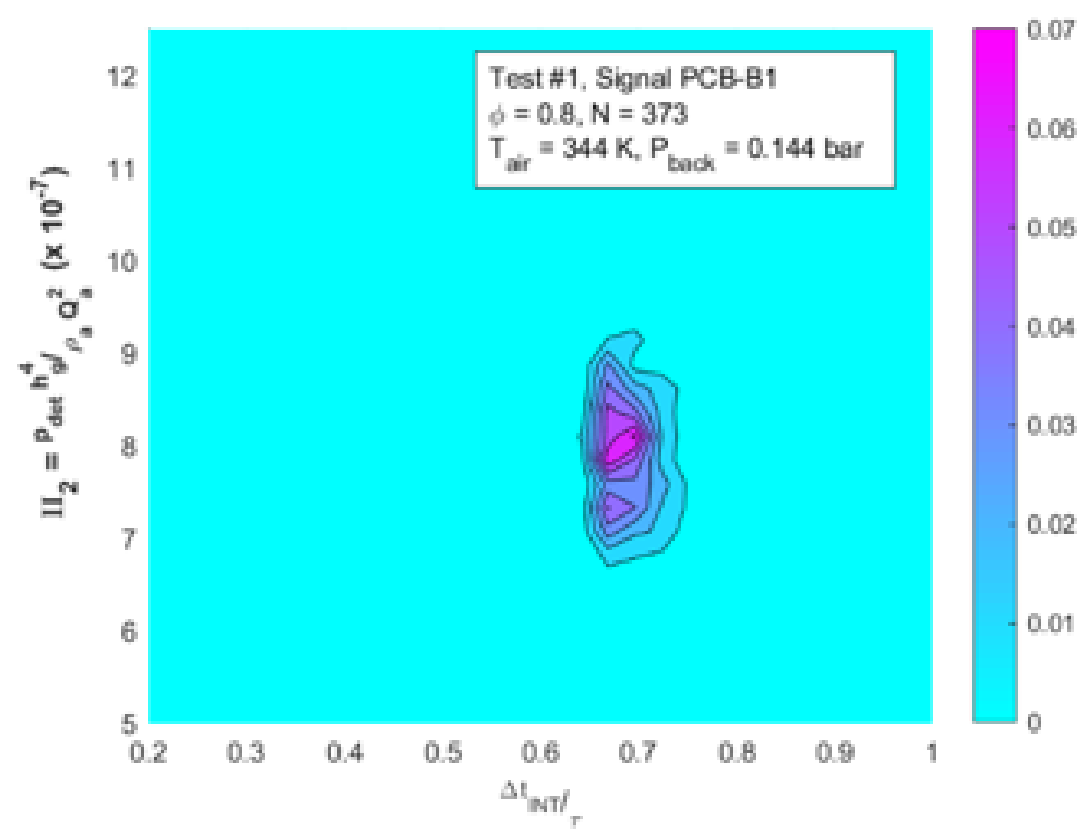

Figure 4.20: Contour plot of dimensionless parameters

The contour plot provides a clearer representation of each test case. Figure 4.20 depicts a single grouping of data and indicates that the system favors a singular operating mode.

To create a better direct comparison to BSRDE data, these data points are averaged together. Prior to averaging, Chauvenet's Criterion is applied to eliminate any outliers in the data. Chauvenet's Criterion defines a probability band, based on the mean value of a normal distribution, within which all samples of a system should reasonably fall within. The first step is to calculate the confidence interval $(C I)$ based on the number of samples in the data set $(N)$. Then, the probability $(P)$ can be found from the confidence interval. These preliminary steps are mathematically depicted in Equation (4.13). 


$$
\begin{gathered}
C I=\left[1-(2 N)^{-1}\right] \\
P=\frac{(1-C I)}{2}
\end{gathered}
$$

Using the standard normal table, the $Z$-value corresponding to the calculated $P$-value can be found. There will be both a positive and negative solution. This value is denoted ' $Z$ '. Finally, the bounds of the probability band defined by Chauvenet's Criterion can be calculated by multiplying the resulting $Z$-value and the sample standard deviation of the system, shown symbolically in Equation (4.14).

$$
\begin{gathered}
Z=Z(P) \\
X_{c h v}=Z \sigma_{s}
\end{gathered}
$$

Thus, by Chauvenet's, for any data point whose deviation from the sample mean exceeds the magnitude of $X_{c h v}$ can be eliminated as an outlier. This theorem assumes a normal distribution and that the measurement is of a single phenomenon. Recall that earlier in this section it was proved that the peak pressure follows a normal distribution.

Finally, a mean signal shape, in addition to the uncertainty in the mean signal determination, can be found and plotted, provided in Figure 4.21. 


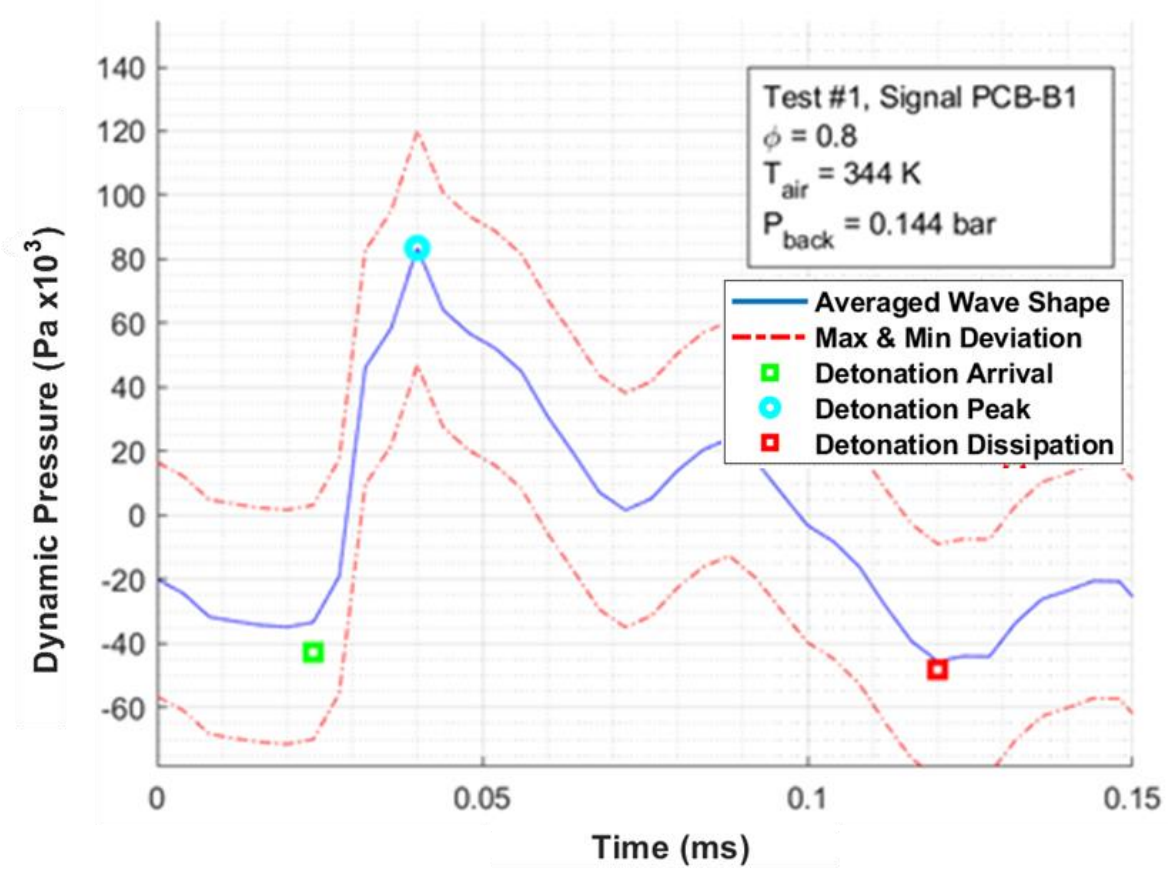

Figure 4.21: Average wave shape plotted with minimum and maximum deviations

With the goal of finding mathematical and graphic methods to relate the LSRDE and BSRDE, an average $\Pi_{2}$ term was determined for each test for the LSRDE. These points were used to generate a comparable graphic (Figure 4.22) to Figure 4.8.

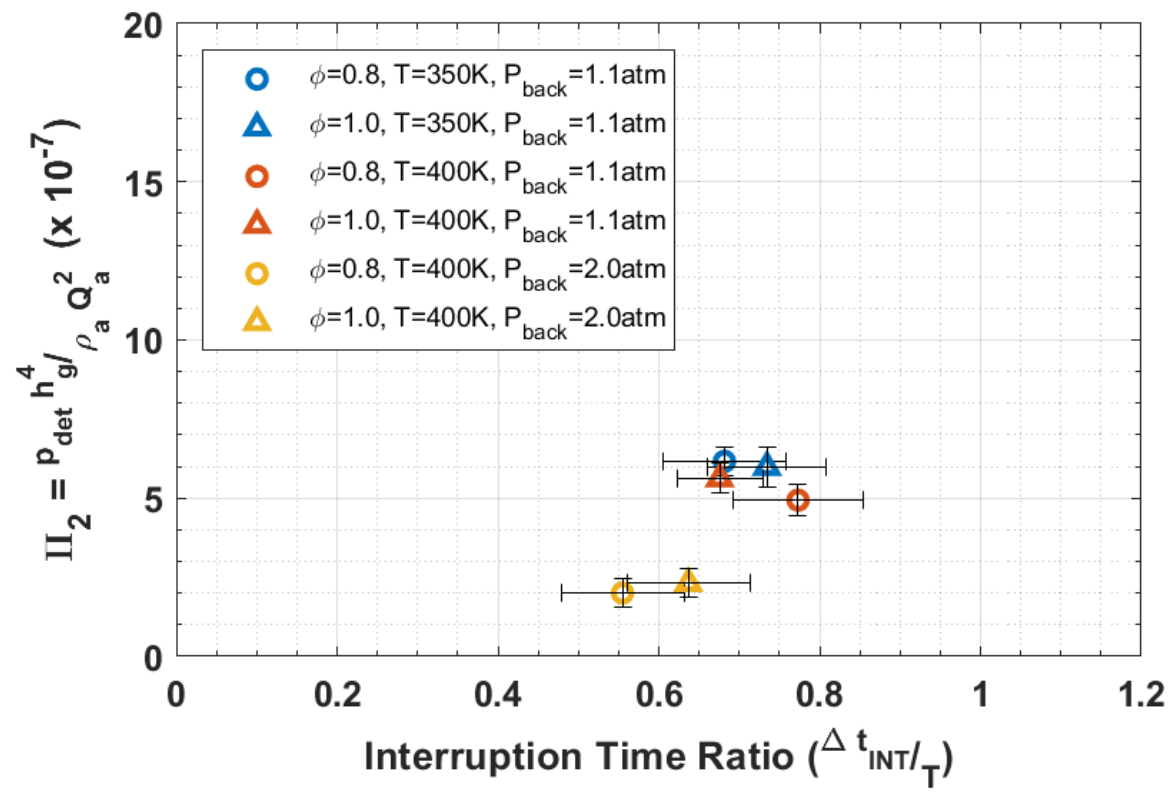

Figure 4.22: $\Pi_{2}$ versus interruption time ratio 
In the LSRDE, equivalence ratio, air supply pre-heat temperature, and back pressure were varied. An identical plot to that of the BSRDE is therefore impossible using this data set. These relationships are ultimately dependent upon the measurement uncertainty and will be discussed further in Section 6.2. This methodology was employed on the following cases, listed in Table 4.3.

Table 4.3: Testing Matrix (Number of cases at each set of conditions). All cases at air supply flow rates of $42 \mathrm{kSCFH} \leq Q \leq 45 \mathrm{kSCFH}$

\begin{tabular}{|c|c|c|c|c|}
\hline & \multicolumn{2}{|c|}{$\mathbf{P}=\mathbf{1 . 1} \mathbf{~ a t m}$} & \multicolumn{2}{c|}{$\mathbf{P}=\mathbf{2 . 0} \mathbf{~ a t m}$} \\
\hline$\phi=0.8$ & $\mathbf{T = 1 5 0 ^ { \circ }} \mathbf{F}$ & $\mathbf{T}=\mathbf{2 5 0} 0^{\circ} \mathbf{F}$ & $\mathbf{T}=\mathbf{1 5 0}^{\circ} \mathbf{F}$ & $\mathbf{T = 2 5 0}{ }^{\circ} \mathbf{F}$ \\
\hline$\phi=1.0$ & 8 & 1 & No Data & 2 \\
\hline
\end{tabular}

\section{Uncertainty}

Measurement uncertainty is paramount to experimental investigations. It gives context to the relevance and significance of any experimental findings. There are generally two sources of uncertainty, bias and precision. Bias encompasses systematic uncertainty. It is comprised of all sources of measurement uncertainty due to instrumentation. Bias uncertainty is generally given for each measurement device as a percentage of full scale.

The precision uncertainty accounts for random error in the system. It constitutes the variance in the repeated measurement of the same system under the same conditions. These values are combined to give a total uncertainty in the measured parameter by finding the square root of the sum of squares.

By assuming that the uncertainty in each of the measurements is random and independent, quadrature can be applied to the maximum likely uncertainty calculation.

$$
\delta q=\sqrt{\left(\frac{\partial q}{\partial x_{1}} \delta x_{1}\right)^{2}+\cdots+\left(\frac{\partial q}{\partial x_{n}} \delta x_{n}\right)^{2}}
$$


This methodology requires individually perturbing the calculated value of ' $q$ ' based on the maximum uncertainty of each measurement device.

In this analysis, only the uncertainty of the $\Pi_{2}$ term is determined. The uncertainty in the interruption ratio is simply a result of sampling error. Considering that the interruption time ratio is calculated from two temporal estimations, the uncertainty would simply be two divided by the sample rate. The inverse of the sample rate gives the time-step between each point in the signal. The maximum uncertainty that could exist is simply double this value which results in $8 \mathrm{~s}$ for LSRDE and 1-8 s for the BSRDE depending on the sample rate used which varies from $250 \mathrm{kHz}$ to $2 \mathrm{MHz}$. This is partially due to the fact that the rise time of the measurement device is sufficiently low enough to obtain the signal peak, so this analysis need not consider it.

\subsection{BSRDE Uncertainty}

The devices contributing to the bias uncertainty within the BSRDE measurements are provided in

Table 5.1. The calculation for arriving at the value for bias uncertainty is given in Appendix A.

\section{Table 5.1: BSRDE Uncertainty contributions to $\Pi_{2}$}

\begin{tabular}{|c|l|l|c|}
\hline & \multicolumn{1}{|c|}{ Measurement } & \multicolumn{1}{|c|}{ Device } & Uncertainty \\
\hline \multirow{2}{*}{$\mathbf{p}_{\mathbf{C H}}$} & Dynamic Channel Pressure & PCB CA 102-B06 & $1.3 \%$ \\
\cline { 2 - 4 } & Atmospheric Pressure & Omega Atmospheric & $0.25 \%$ \\
\hline \multirow{2}{*}{$\boldsymbol{\rho}_{\mathbf{a}}$} & Atmospheric Pressure & Omega Atmospheric & $0.25 \%$ \\
\cline { 2 - 4 } & Plenum Temperature & K Type TC & $0.75 \%$ \\
\hline \multirow{2}{*}{$\mathbf{Q}_{\mathbf{a}}$} & Channel Air & Omega Air 200 Supply & $1.0 \%$ \\
\cline { 2 - 4 } & Channel Air & Alicat Air 500 Supply & $0.2 \%$ \\
\hline
\end{tabular}

After perturbing the dimensionless pressure value for each uncertainty contribution, the bias uncertainty was found to be: 


$$
\frac{\delta \Pi_{2}}{\Pi_{2}}=1.67 \%
$$

Since the BSRDE is built to reproduce a consistent detonation to be measured under varying geometric and air plenum supply conditions, precision error can be calculated for each set of testing conditions. To do this, the Student-t distribution is used to estimate precision uncertainty. The mean, standard deviation, and number of samples are needed to complete this calculation. The tvariable is determined by the degrees of freedom of the system and the desired confidence interval, which is $95 \%$ here. The equation to find the probability is given in Equation (5.3).

$$
P=\frac{t \cdot \sigma_{s}}{\mu \sqrt{N-1}}
$$

The uncertainty for the BSRDE under various flow rate conditions are provided in Table 5.2.

Table 5.2: BSRDE Uncertainty for Various Air Supply Flow Rates

\begin{tabular}{|c|c|c|c|}
\hline & 300 SLPM & 500 SLPM & 700 SLPM \\
\hline Mean & $1.20 \mathrm{E}-03$ & $3.49 \mathrm{E}-04$ & $1.36 \mathrm{E}-04$ \\
\hline Standard Deviation & $4.65 \mathrm{E}-04$ & $1.22 \mathrm{E}-04$ & $4.71 \mathrm{E}-05$ \\
\hline $\mathbf{N}$ & 19 & 24 & 35 \\
\hline $\mathbf{t}$ & 1.729 & 1.711 & 1.697 \\
\hline$\delta \Pi_{2} / \Pi_{2}$ (Precision) & $15.4 \%$ & $12.2 \%$ & $10.0 \%$ \\
\hline$\delta \Pi_{2} / \Pi_{2}$ (Bias) & $1.7 \%$ & $1.7 \%$ & $1.7 \%$ \\
\hline$\delta \Pi_{2} / \Pi_{2}$ & $15.5 \%$ & $12.3 \%$ & $10.1 \%$ \\
\hline
\end{tabular}

The BSRDE uncertainty results are significant. Variation can occur within the pre-detonator tube, especially concerning mixing. Therefore, some of the contribution of this precision error could be real variation in the system performance. For the purpose of this study, which cannot attempt to further analyze the precision uncertainty, consider the magnitude of overall uncertainty in succeeding analyses and conclusions. 


\subsection{LSRDE Uncertainty}

To obtain the uncertainty in the desired parameter of dimensionless pressure for the LSRDE, the sources of uncertainty need to be considered. Table 5.3 summarizes the sources of measurement uncertainty contributing to $\Pi_{2}$.

Table 5.3: LSRDE Uncertainty contributions to $\Pi_{2}$

\begin{tabular}{|c|l|l|c|}
\hline & \multicolumn{1}{|c|}{ Item } & \multicolumn{1}{|c|}{ Model Number } & Uncertainty \\
\hline \multirow{2}{*}{ pсH } & Dynamic Channel Pressure & PCB CA 102-B06 & $1.0 \%$ \\
\cline { 2 - 4 } & Back Pressure & Rosemount 1151GP8 & $0.08 \%$ \\
\hline \multirow{5}{*}{$\mathbf{a}$} & Plenum Supply Pressure & Kulite 8442-4-140 & $0.5 \%$ \\
\cline { 2 - 4 } & Plenum Temperature & Omega KQXL-116U-6 & $0.75 \%$ \\
\hline \multirow{5}{*}{ Qa } & Differential Pressure (1) & & $0.1 \%$ \\
\cline { 2 - 4 } & Reference Pressure for Flow Correction & Rosemount 1151GP8 & $0.08 \%$ \\
\cline { 2 - 4 } & Reference Temperature for Flow Correction & K-Type TC & $0.75 \%$ \\
\cline { 2 - 4 } & Differential Pressure (3) & & $0.1 \%$ \\
\cline { 2 - 4 } & Reference Pressure for Flow Correction & Rosemount 1151GP8 & $0.08 \%$ \\
\cline { 2 - 4 } & Reference Temperature for Flow Correction & K-Type TC & $0.75 \%$ \\
\cline { 2 - 4 } & Differential Pressure (3) & & $0.1 \%$ \\
\cline { 2 - 4 } & Reference Pressure for Flow Correction & Rosemount 1151GP8 & $0.08 \%$ \\
\cline { 2 - 4 } & Reference Temperature for Flow Correction & K-Type TC & $0.75 \%$ \\
\hline
\end{tabular}

In a system as highly variable as an RDC, it is inaccurate to calculate the precision uncertainty separately. The PCB measurement devices, which are the only component measuring the highly variable and dynamic nature of the system, are in fact calibrated by the manufacturer in a way that considers both bias and precision uncertainty. This is done by testing a repeatable event and determining the variation in each measurement. The precision uncertainty surrounding the remainder of the measurement devices is ignored as each measured variable is binned in the analysis to better compile results and simplify analysis. The total uncertainty in the dimensionless pressure term is given in Equation (5.4). 


$$
\frac{\delta \Pi_{2}}{\Pi_{2}}=1.09 \%
$$

Typically, precision uncertainty has to do with the variability between samples of the same measurement. In calculating the average $\Pi_{2}$ term for each test, each "wave" would be considered a sample. However, this assumes that the phenomena within the system being measured is repeatable. Since the RDC is inherently variable through the duration of a test, precision uncertainty is not further investigated, and instead, system variance is discussed. To present the variance in a similar fashion as the uncertainty, a ratio of sample standard deviation to the sample mean, such as in Equation (5.5), is provided.

$$
\text { system variation }=\frac{\sigma_{s}}{\mu}
$$

It is unnecessary here to present the variation within each test. The important conclusion is that there is tangible variation within the RDC system that should not be included in the uncertainty as that would suggest that the findings are incorrect. Instead, measurement of a highly dynamic and variable environment is being analyzed, and while it is important to recognize variability, it is not amenable to merge real system variance with measurement uncertainty. A representative case is presented in Table 5.4.

Table 5.4: LSRDE Variation

\begin{tabular}{|c|c|}
\hline Mean & $5.62 \mathrm{E}-09$ \\
\hline Standard Deviation & $6.52 \mathrm{E}-08$ \\
\hline$\sigma_{\mathrm{s}} / \mu$ & $11.6 \%$ \\
\hline
\end{tabular}




\section{Results and Discussion}

For both rigs, repeatability and stability within the system will be presented and discussed first in the form of stacked wave plots and averaged wave shapes. In the case of the LSRDE, this will also include spectrogram plots. Succeeding analysis will entail a discussion on system operation. This discussion will center around the interruption time and dimensionless pressure terms, observing trends in their relationship and speculating on the influence of each term within the dimensionless pressure.

\subsection{BSRDE Results and Discussion}

The bench scale RDE ideally provides a repeatable shock into the linearized detonation channel. Effects of geometric alterations have previously been studied [26]. Presented here are the volumetric flow rate influences on the dimensionless parameters developed in Section 4.1.2. Therefore, results begin with the wave stacking plots for each respective air supply flow rate, as outlined in Section 4.1.3.

Figure 6.1 shows that the width of the peaks, and therefore interruption times, are fairly consistent throughout the testing conditions. There does appear to be some variability in the peak pressures, however. Additionally, the shape of the wave varies in regards to rise time and variation. Some tests appear to more closely resemble a von Neumann peak, while others depict a more gradual rise. 


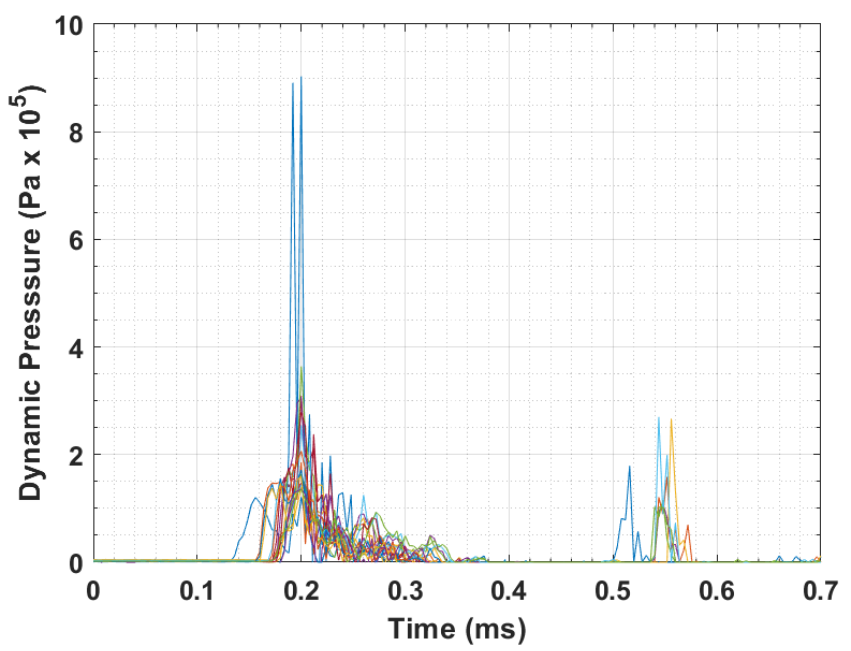

(a) 300 SLPM

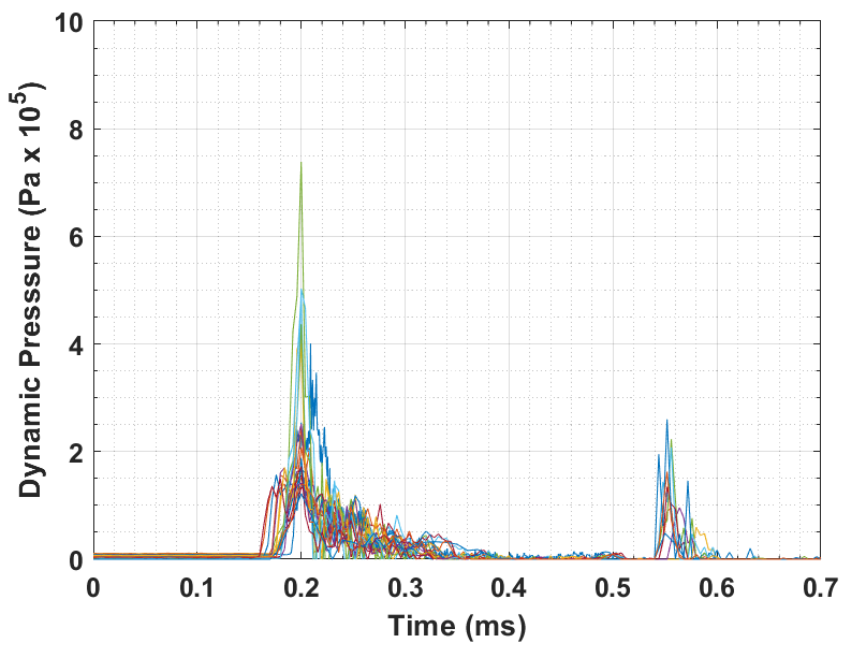

(b) 500 SLPM

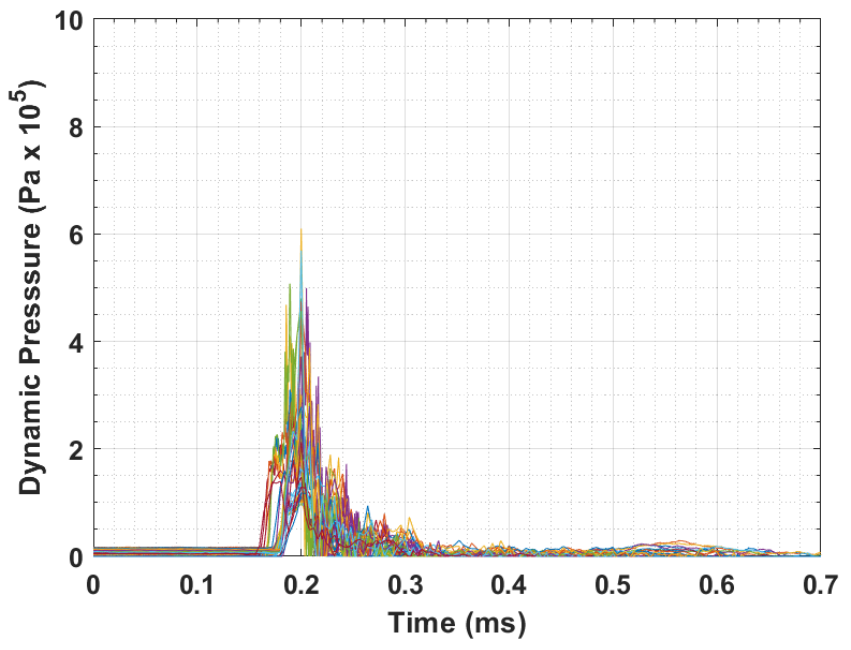

(c) 700 SLPM

Figure 6.1: BSRDE Wave Stacking 
Previously, in Section 4.1.3, the variation within each parameter contributing to the dimensionless pressure is discussed. These plots are provided here, for convenience.

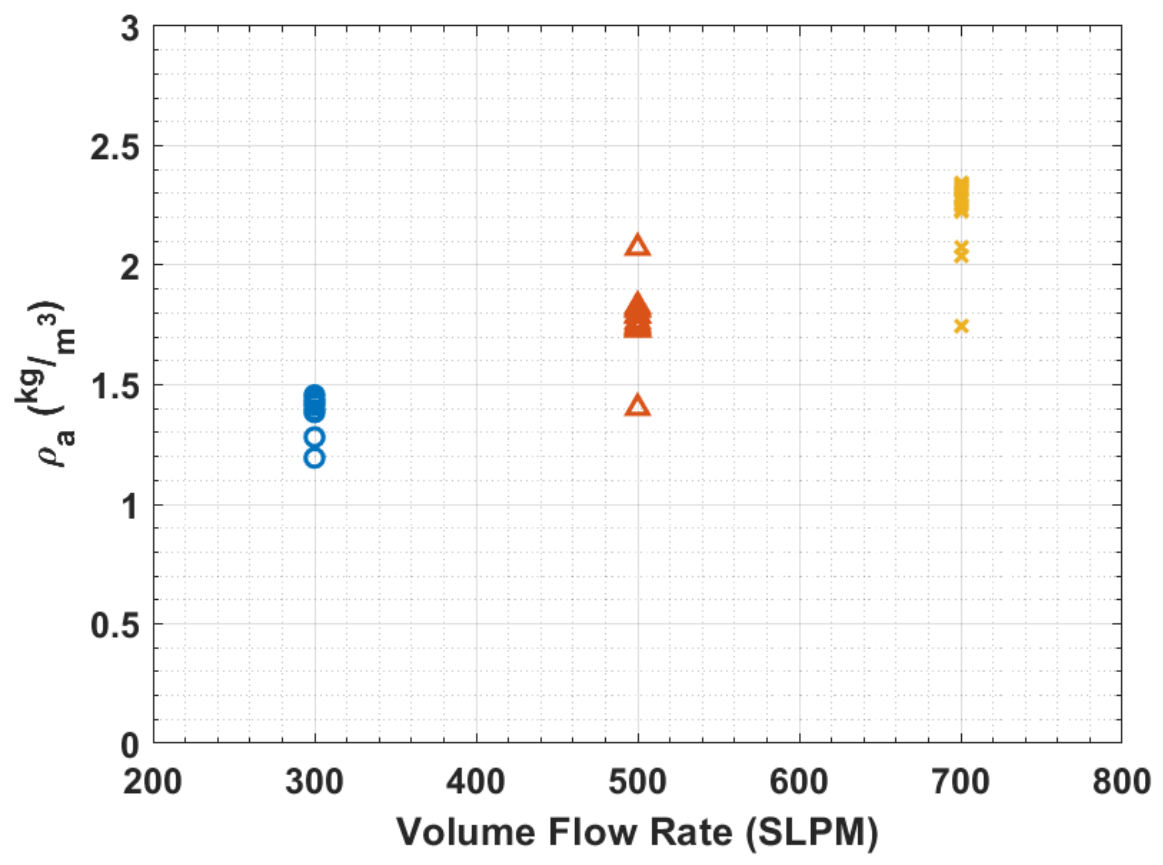

Figure 6.2: Density Variation

In Figure 6.2, the density variation of the air supply cases is considerable. This is due to the change in plenum pressure as a result of increased flow rate which is used to calculate density via the ideal gas law. Higher flow rates require higher supply pressures to force the fluid downstream.

The variation in the peak pressure parameter is given in Figure 6.3. Ideally, the peak pressures would align more closely since a design consideration of the BSRDE facility is to reproduce a consistent detonation wave. There are a few data points that may be questioned as outliers. This could not be proven, however. Even the tighter grouping between $200-400 \mathrm{kPa}$ is distributed over a range of 2 atmospheres 


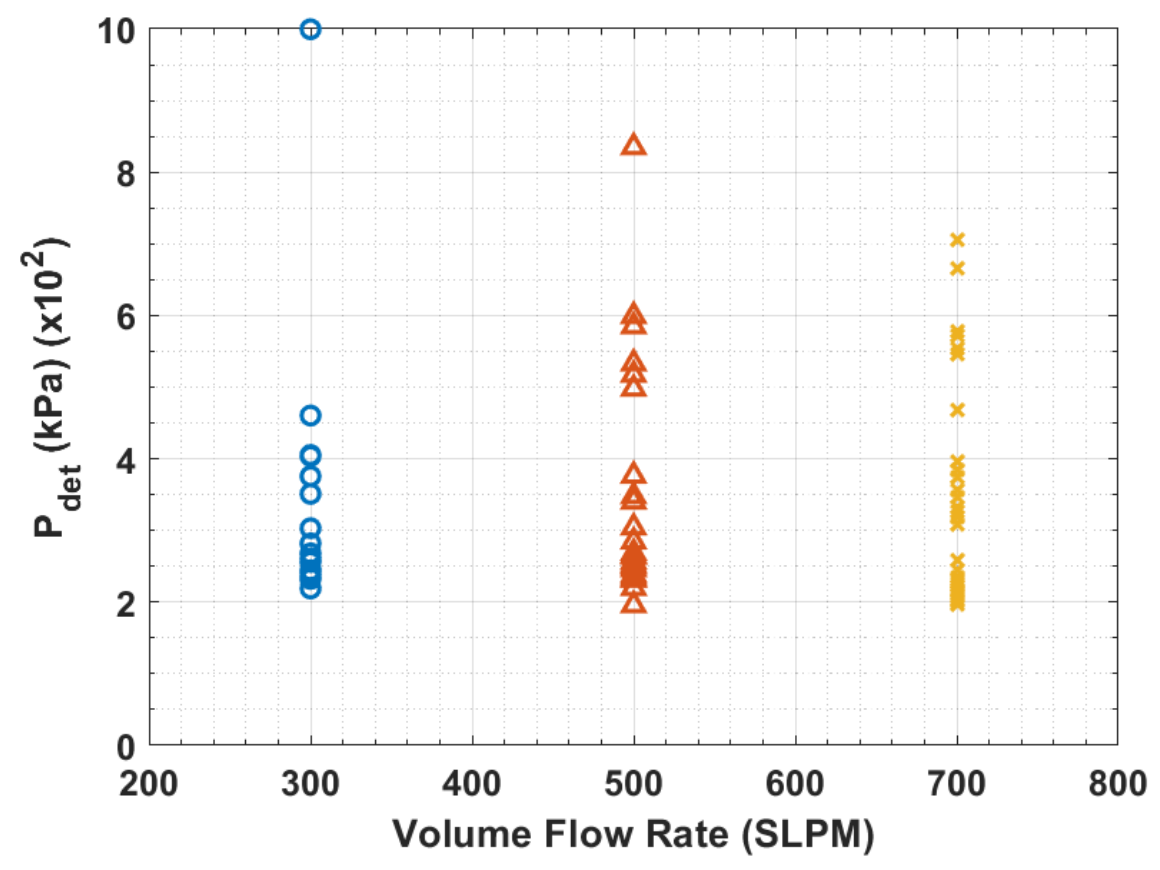

Figure 6.3: Detonation Pressure Variation

Plotting the peak channel pressures and interruption times against each other for all tests yields Figure 6.4. There are no obvious patterns or relationships. In fact, the data appears to be quite sporadic and random.

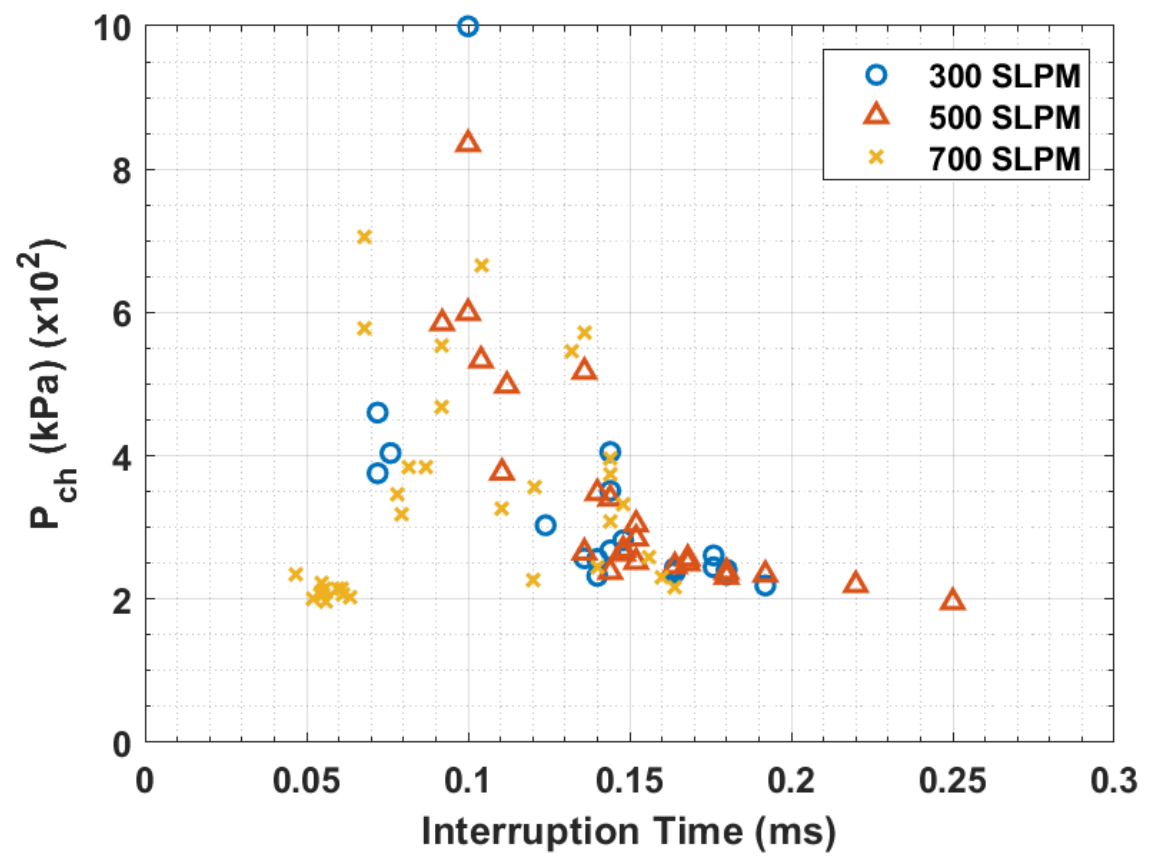

Figure 6.4: Peak Pressure vs Interruption Time 
Figure 6.5, which compares dimensionless pressure and interruption time ratio, presents a different message.

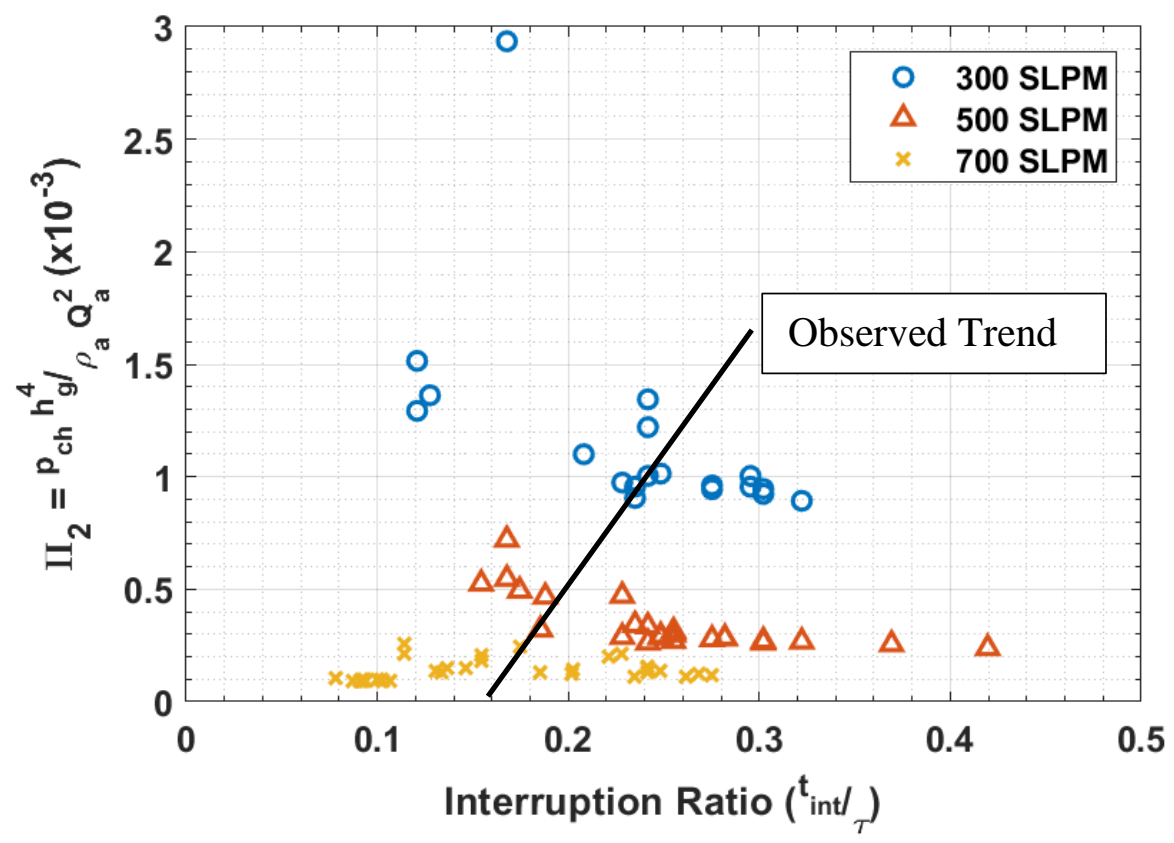

Figure 6.5: Dimensionless Pressure, $\Pi_{2}$ versus Interruption Ratio

The trend seen above shows a layering that organizes higher flow rates, 700 SLPM, at the bottom, and lower flow rates, 300 SLPM, at the top. There is also a slight trend in higher flow rates toward the left, denoting lesser interruption ratios, and lower flow rates toward the right. Conceptually, these trends make sense. For the $\Pi_{2}$ term plotted along the y-axis, the detonation pressure and air injection height should be similar for each test. It is seen above that air supply density changes correlate with changes in air supply flow rates due to the increased supply pressure, thus the higher the flow rate, the lower the value of the $\Pi_{2}$ term. Additionally, with higher flow, it is reasonable to expect that the influence of the detonation will be "pushed" out by the larger supply flow, thereby decreasing the interruption ratio. This is reasonable to conclude in this linearized rig, however in an RDC, increased supply air likely would increase mixing, thus increasing detonation strength which could generate a larger pressure feedback. It is possible that greater mixing 
encourages multi-wave modes, thus reducing peak pressures and therefore feedback. RDCs add significant complexity that is redacted in this experiment.

\subsection{LSRDE Results and Discussion}

Results of the LSRDE analysis will be provided in three subsections. First, the stability of the system will be discussed using spectrograms, full-width at half-maximum (FWHM), and average wave shape. Next, a more in-depth discussion into the performance of the system relative to the parameters defined previously will be presented. Finally, a broad summary of the LSRDE performance across all testing conditions will be provided in order to develop conclusions on the impact of testing conditions along with meeting the goal of developing relationships between the two experimental rigs.

\subsubsection{Stability}

Figures 6.6-6.11 depict a representative case for each set of conditions. The spectrogram in location (a) shows the stability of the system. The figure in (b) shows the wave characteristics for the filtered signal. This details how the various parameters were calculated and provides a visual check on the performance of the code. It is seen that some peaks were missed. This is due to complexity of the system and lack of robustness in the code. Shock reflections and variability make it difficult to define conditions that will always succeed in peak finding. Missing peaks can also be indicative of difficulty in finding the beginning or end of the wave. If either mark were not found, the individual waveform would be discounted from further analysis. Figure (c) shows each of the wave shapes determined in figure (b) aligned by peak location. These wave forms are averaged over each sampling point to give an average wave form for the testing conditions, presented in Figure (d). 


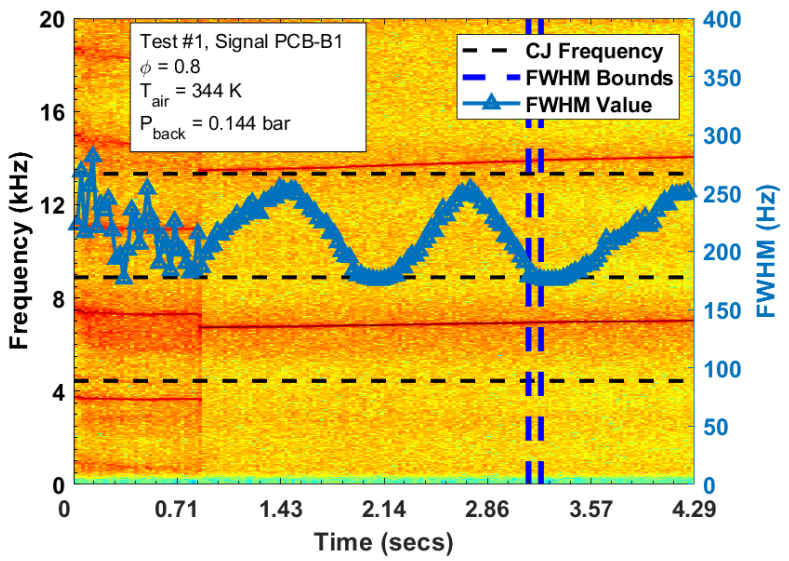

(a) Spectrogram

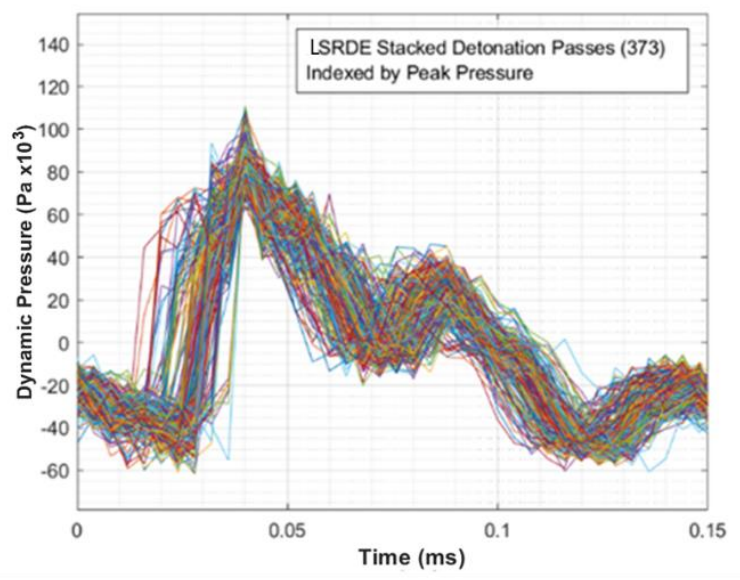

(c) Stacked Detonation Waves

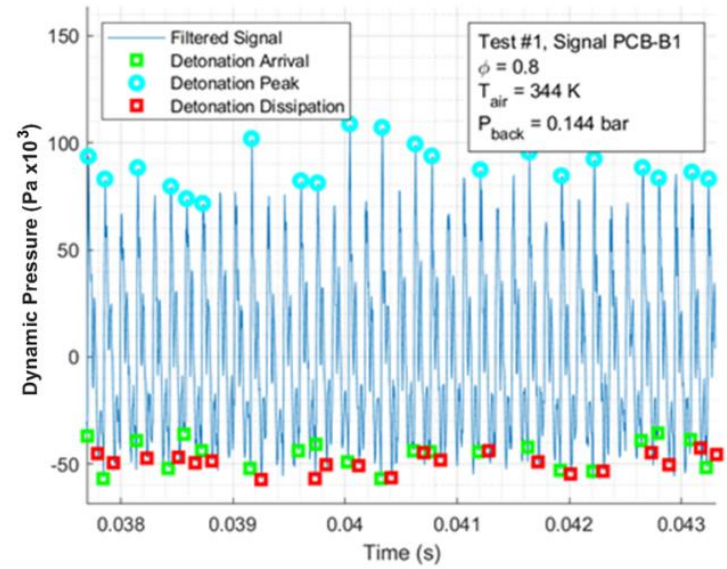

(b) Detonation Characteristics

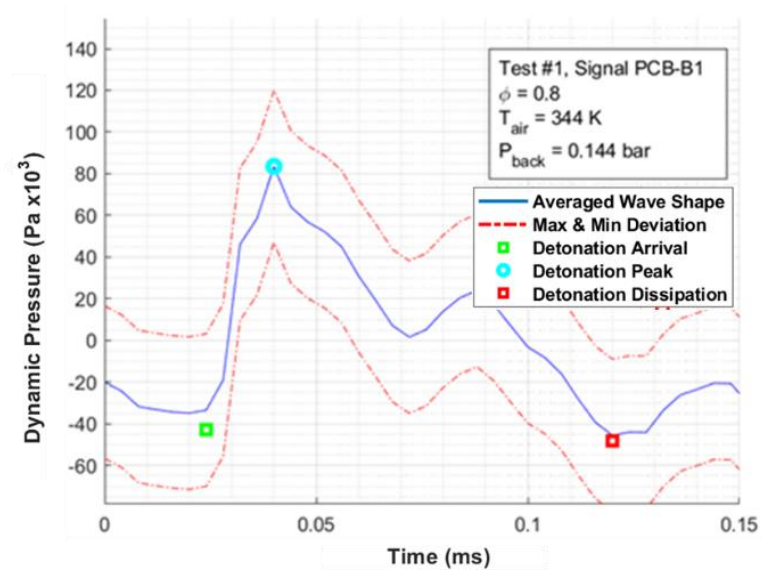

(d) Averaged Detonation Wave Form

Figure 6.6: $Q=40,000 \mathrm{SCFH}, \phi=0.8, T=350 \mathrm{~K}, P=1.1 \mathrm{~atm}$

Figures 6.6 and 6.7 show the difference in RDC operation when the equivalence ratio is increased while keeping the back pressure, pre-heat temperature, and air flow rate the same. By comparing the figures in (a), it is clear that both operate in a two-wave mode. This is determined by the most intense sharp red line in the spectrogram aligning between the first and second black dotted lines, indicating the $\mathrm{CJ}$ frequency for one and two wave operation, respectively.

In Figures 6.6 and $6.7(\mathrm{~d})$, it appears that the $\phi=0.8$ case (Figure 6.6) has a slightly shorter interruption time and is more clearly impacted by a shock reflection. The peak channel pressures appear to increase with equivalence ratio. Additionally, there is a much slower rise in the signal 
for the stoichiometric case (Figure 6.7d). These observations are likely a result of better mixedness in the reactant mixture for the stoichiometric case.

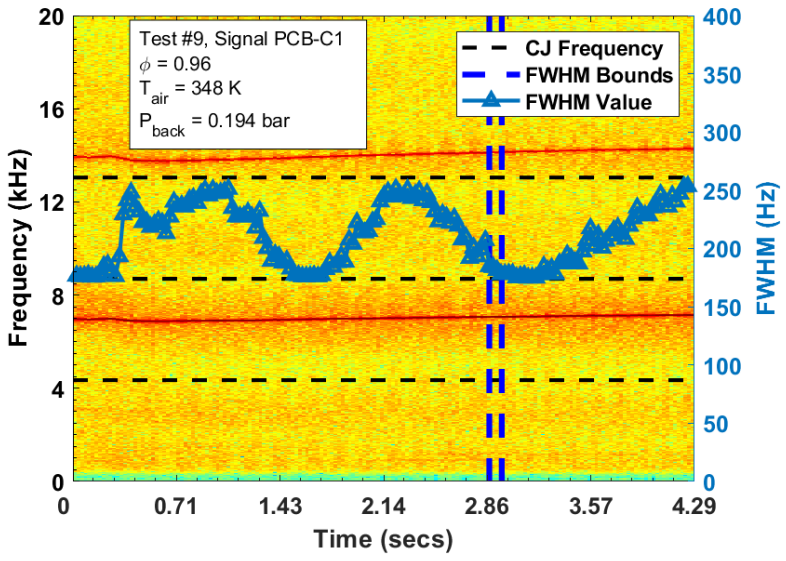

(a) Spectrogram

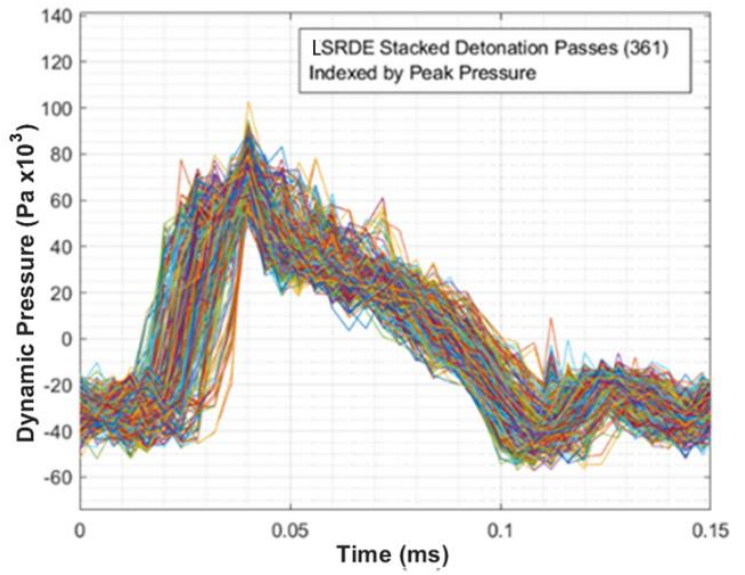

(c) Stacked Detonation Waves

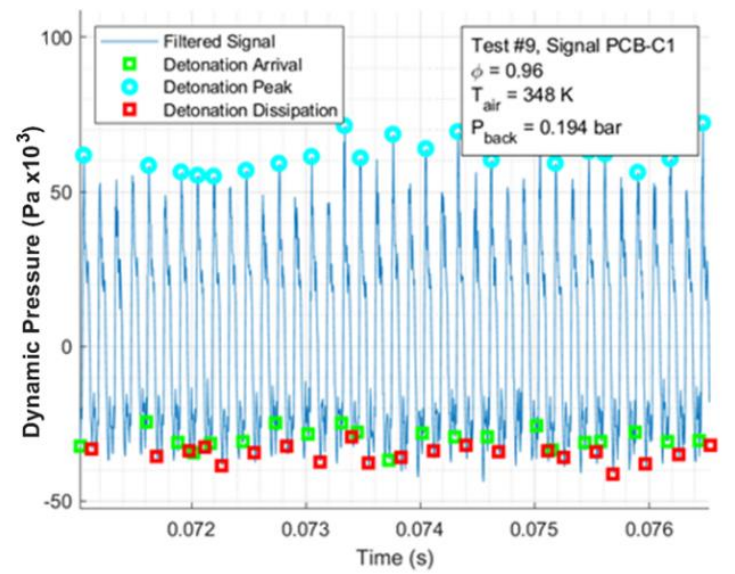

(b) Detonation Characteristics

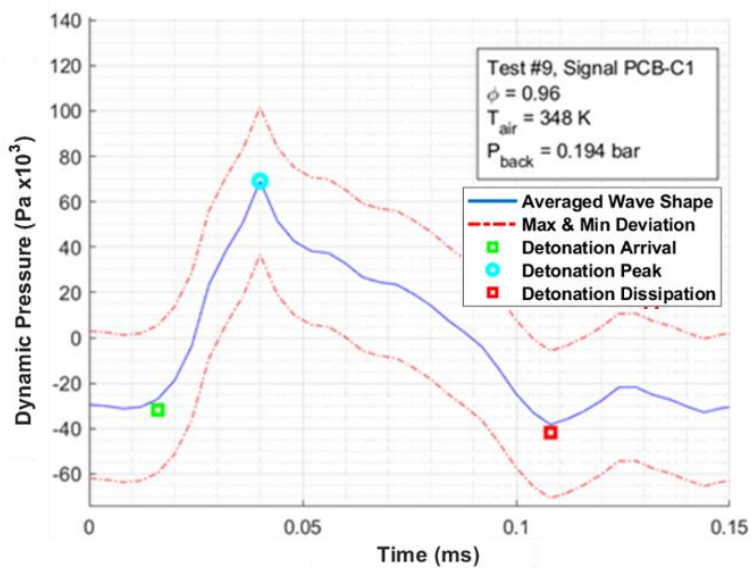

(d) Averaged Detonation Wave Form

Figure 6.7: $Q=40,000 \mathrm{SCFH}, \phi=1.0, T=350 \mathrm{~K}, P=1.1 \mathrm{~atm}$

Figures 6.8 and 6.9 depict the same comparisons as Figures 6.6 and 6.7, however the pre-heat temperature is increased. Similarly, both sets of data depict steady, two-wave operation. By comparing Figures 6.8(d) and 6.9(d), it appears that interruption time and peak pressure both decrease slightly with increased equivalence ratio. 


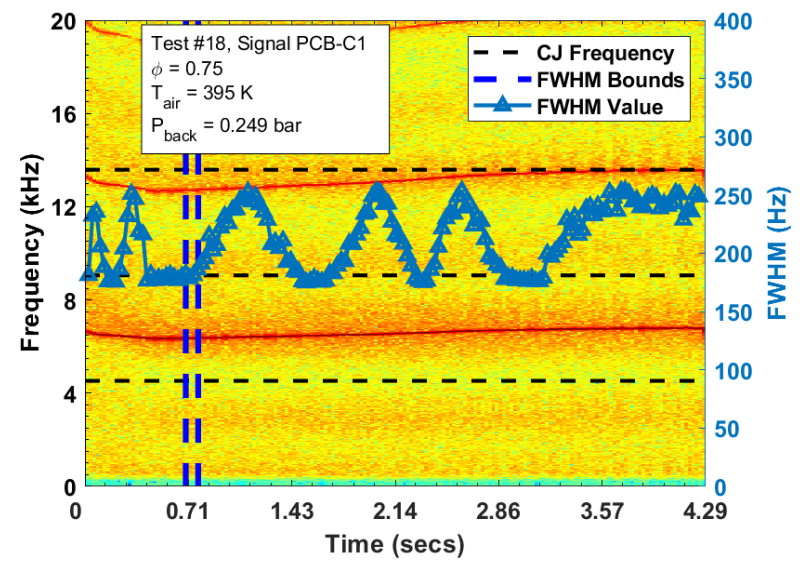

(a) Spectrogram

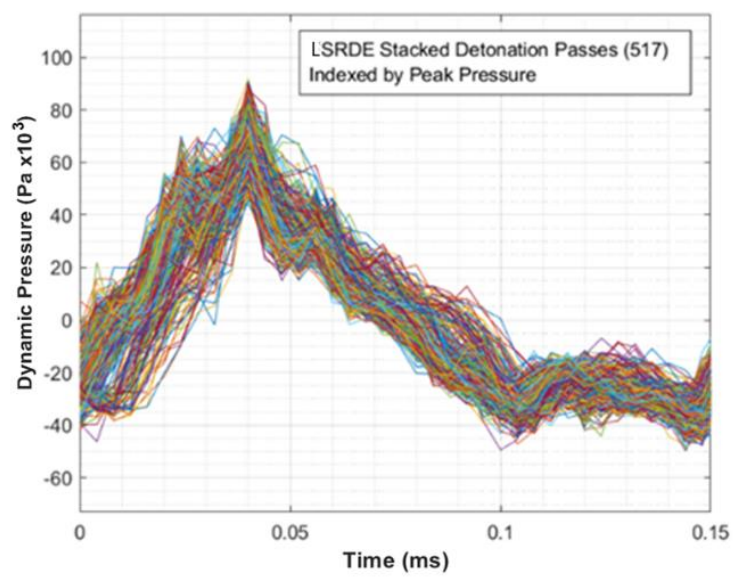

(c) Stacked Detonation Waves

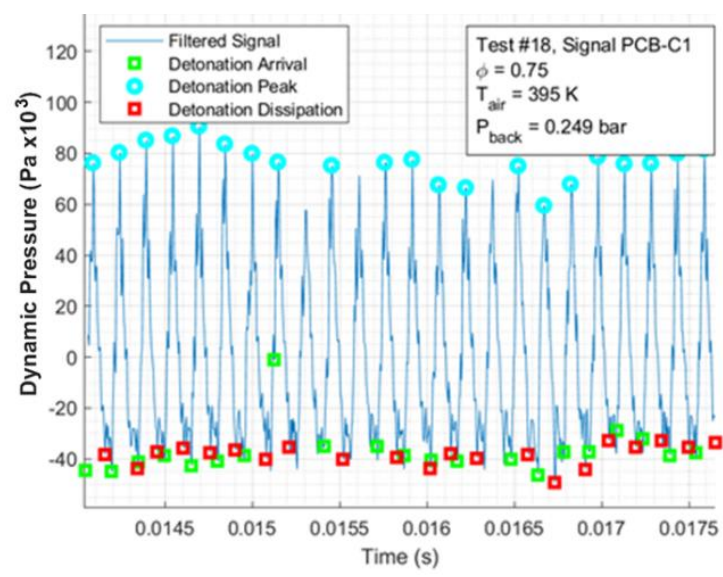

(b) Detonation Characteristics

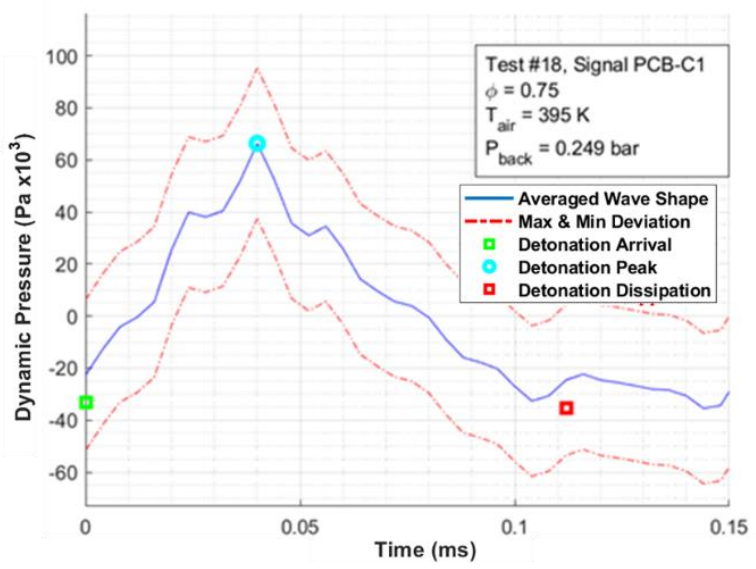

(d) Averaged Detonation Wave Form

Figure 6.8: $Q=40,000 \mathrm{SCFH}, \phi=0.8, T=400 \mathrm{~K}, P=1.1 \mathrm{~atm}$

Both in Figures 6.8(d) and 6.9(d), there is a slow rise to the peak, however in the lower equivalence ratio case (Figure 6.8d), there is greater variability and a preliminary peak. 


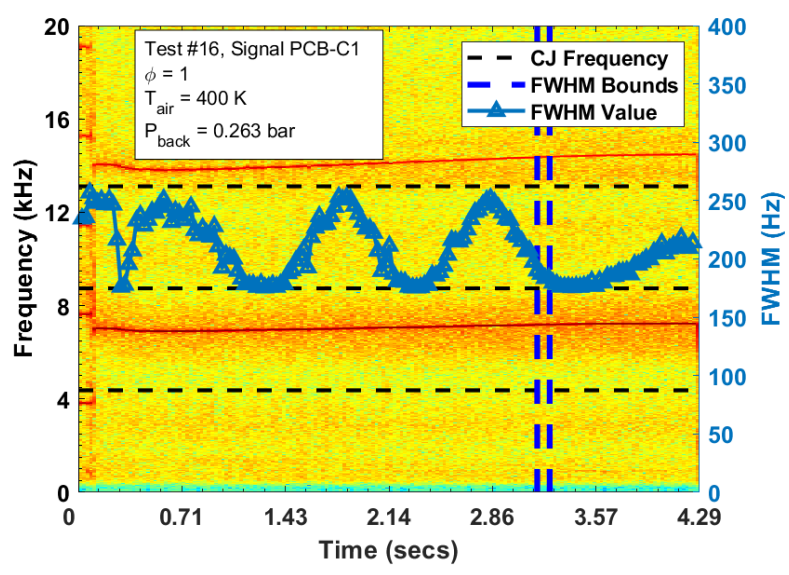

(a) Spectrogram

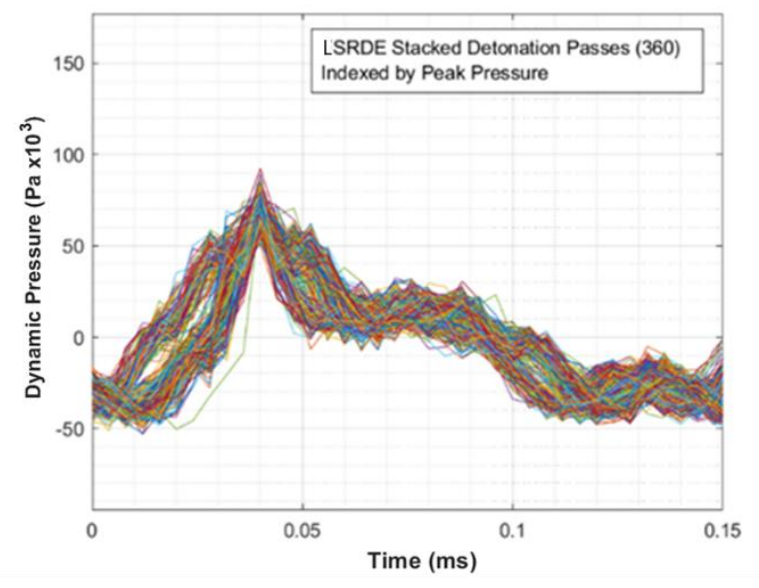

(c) Stacked Detonation Waves

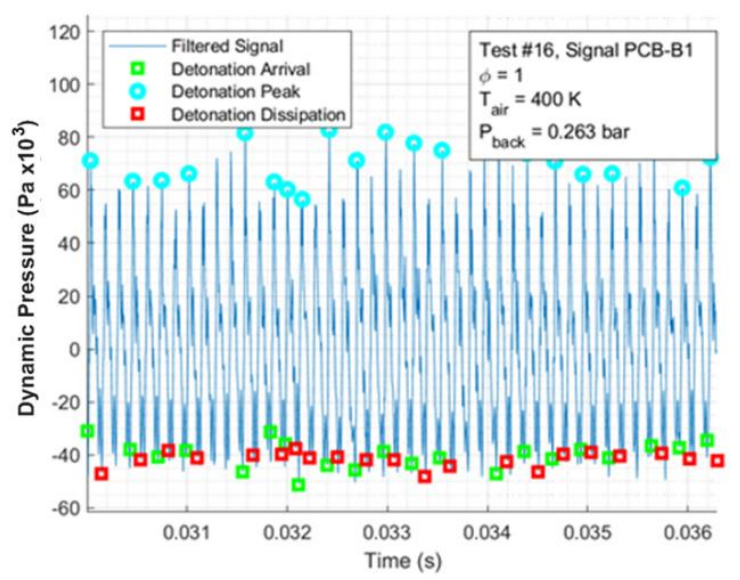

(b) Detonation Characteristics

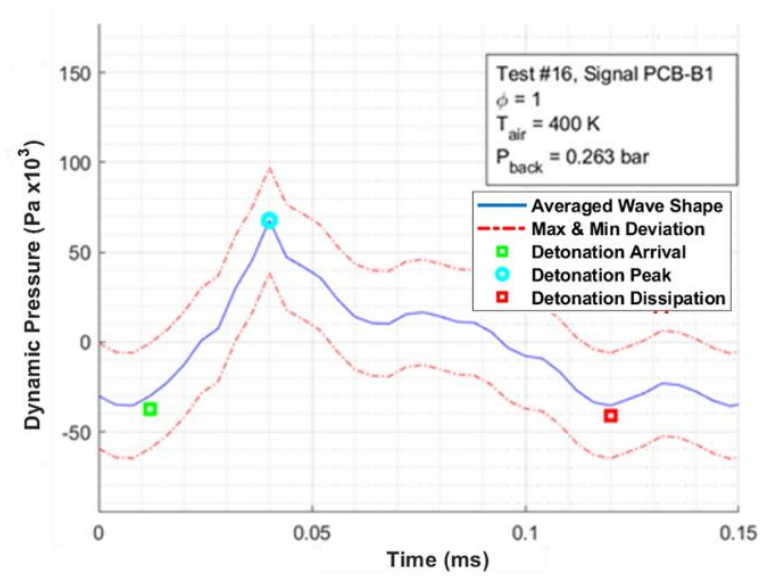

(d) Averaged Detonation Wave Form

Figure 6.9: $Q=40,000 \mathrm{SCFH}, \phi=1.0, T=400 \mathrm{~K}, P=1.1 \mathrm{~atm}$

Finally, Figures 6.10 and 6.11 compare two equivalence ratio cases at elevated pre-heat temperature and back pressure. The spectrogram in Figure 6.10(a) is unclear. Reflecting upon the explanation of spectrograms in Section 4.2, the noise, or lack of sharpness and definition in the contours, would suggest instability or unsteadiness in the signal. There appears to be similar intensity in the spectrogram at $\sim 2.8 \mathrm{kHz}$ and $\sim 4.8 \mathrm{kHz}$, especially at the beginning of the test. As time passes, the frequency band at $\sim 4.8 \mathrm{kHz}$ increases intensity relative to the lower band. This finding indicates that the RDC is operating in a two-wave mode in which one wave is stronger than the other. Figure 6.11(a) shares this result and is a much clearer example. The FWHM values overlaid on the spectrogram indicate highly unsteady operation in the middle of the test. Figures 
6.10(b) and 6.11(b) better portray the instability indicated by the spectrogram. There is a clear pattern in relatively larger and relatively smaller pressure peaks.

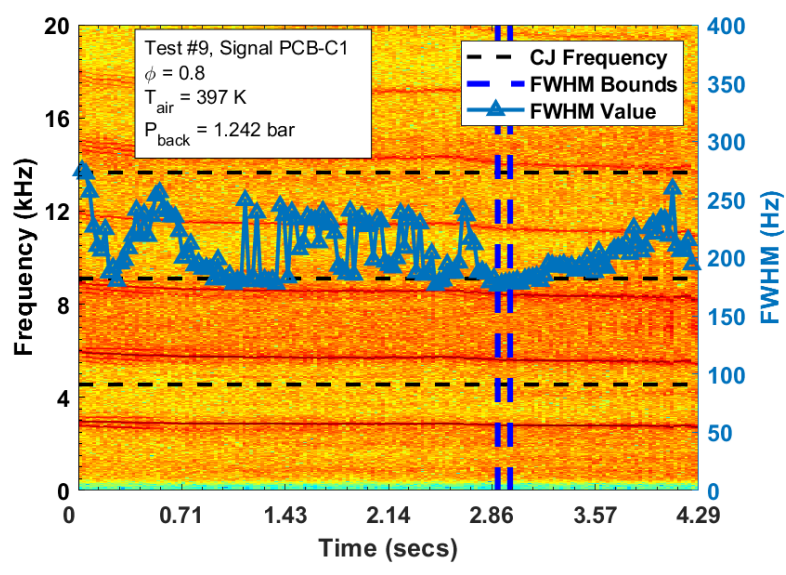

(a) Spectrogram

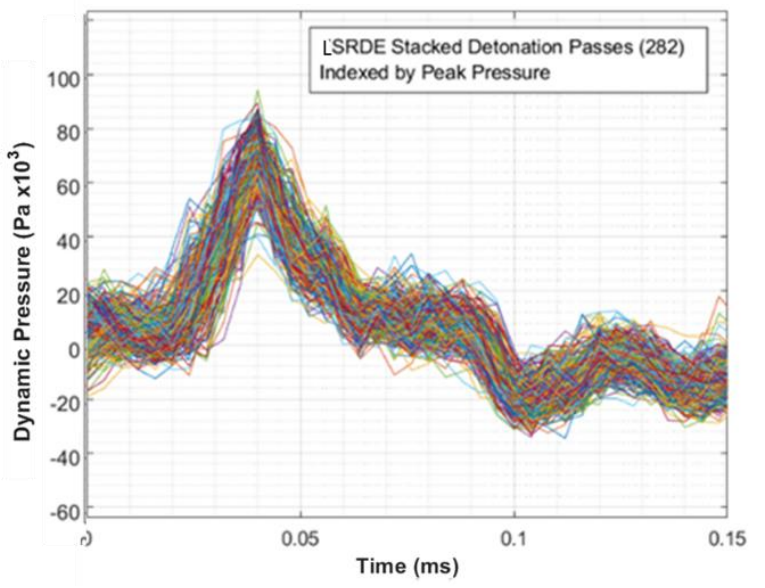

(c) Stacked Detonation Waves

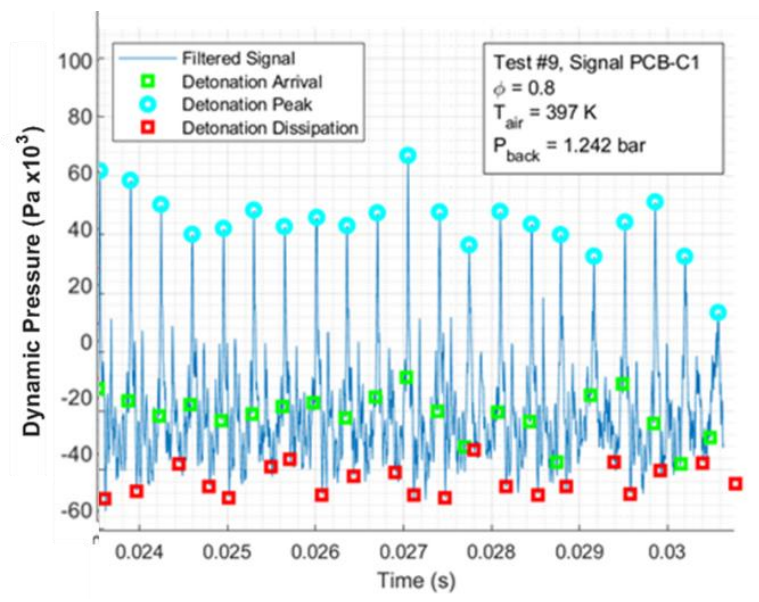

(b) Detonation Characteristics

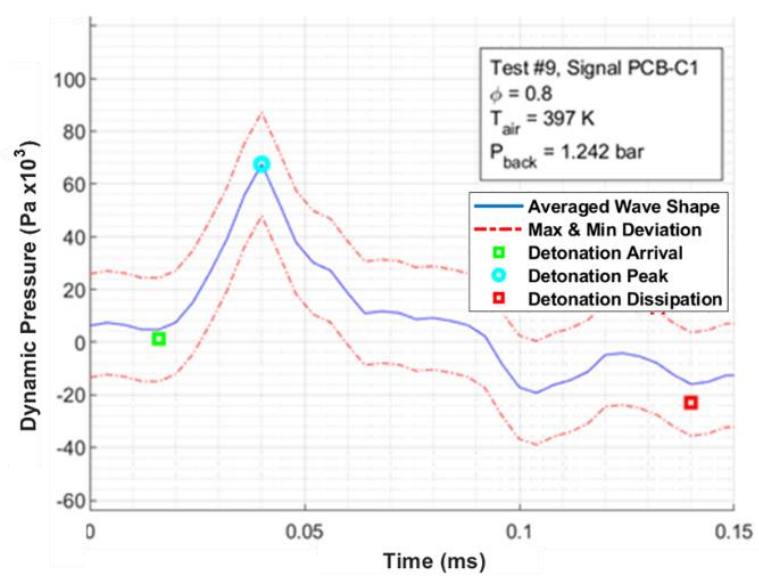

(d) Averaged Detonation Wave Form

Figure 6.10: $Q=40,000 \mathrm{SCFH}, \phi=0.8, T=400 \mathrm{~K}, P=2.0 \mathrm{~atm}$

The existence of a two-wave mode with uneven detonation strengths will significantly affect the results presented in the following section. The MATLAB routine assumes that all waves propagating through the system at any given instant in time are nearly identical. By performing a data reduction under this assumption, results such as Figure 6.11(c) are produced. This figure appears to contain significant variability. Unfortunately, the two apparent groupings of wave forms, where peaks are $\sim 50 \mathrm{kPa}$ and $\sim 120 \mathrm{kPa}$, cannot be further investigated in this work. It could 
be the case that these two groupings closely describe each of the two waves present in the spectrogram, however that investigation is not possible at the present time.

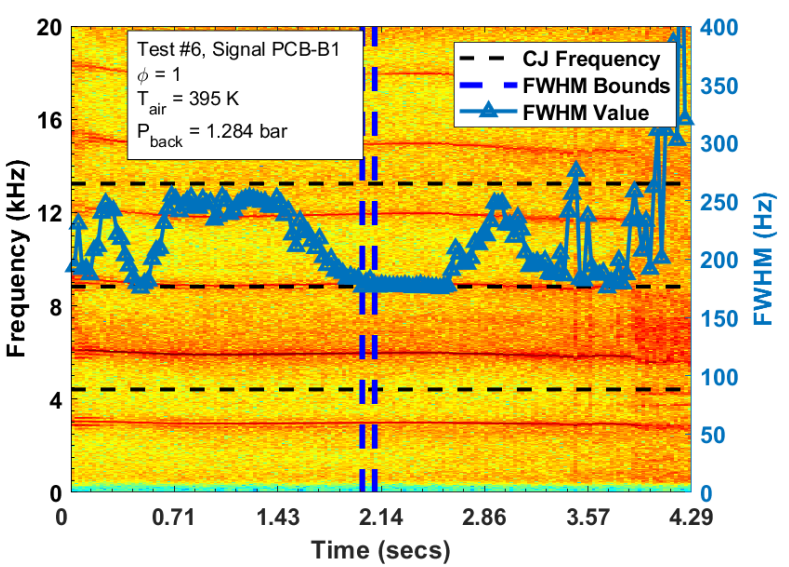

(a) Spectrogram

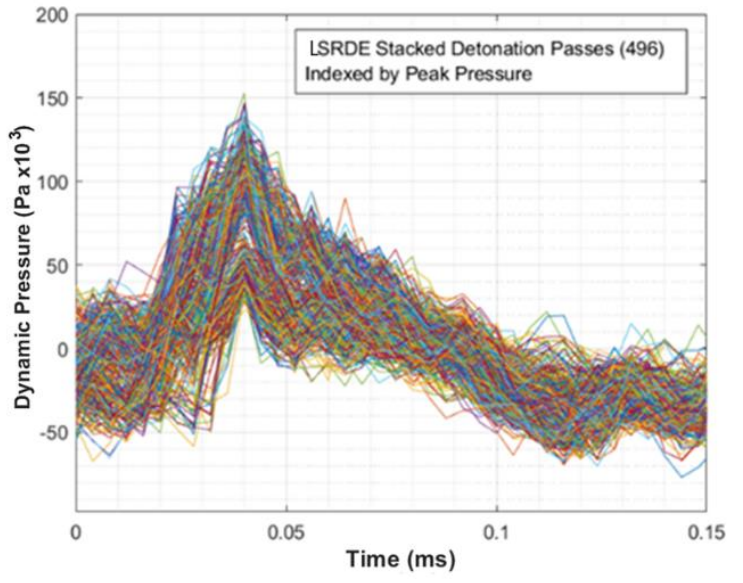

(c) Stacked Detonation Waves

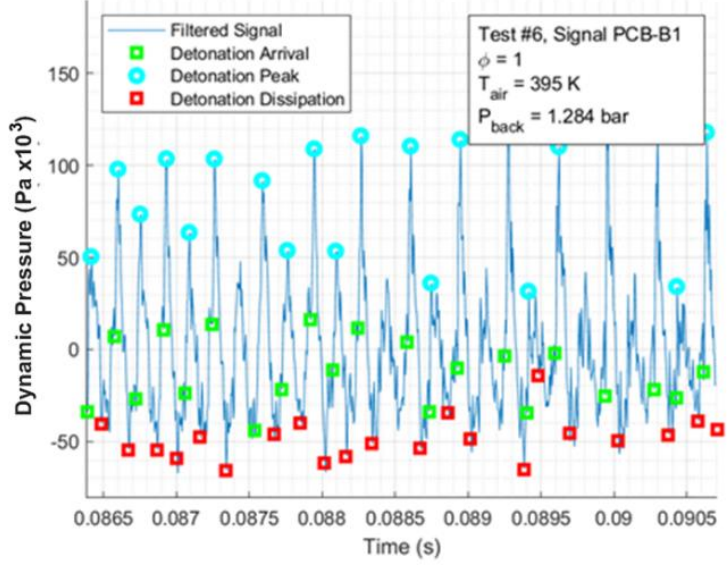

(b) Detonation Characteristics

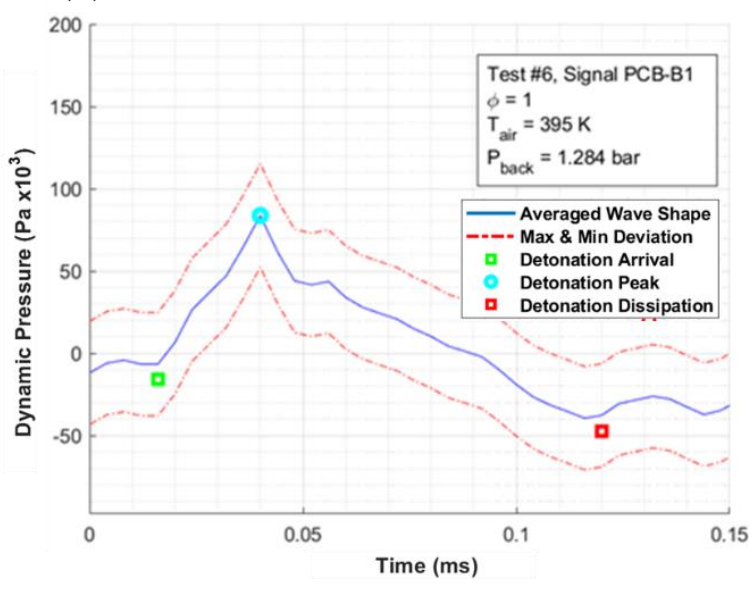

(d) Averaged Detonation Wave Form

Figure 6.11: $Q=40,000 \mathrm{SCFH}, \phi=1.0, T=400 \mathrm{~K}, P=2.0 \mathrm{~atm}$

\subsubsection{Test Performance Analysis}

Having analyzed the stability of the LSRDE under various conditions, the performance of the RDC under each testing condition can be discerned and discussed by elaborating on the previous section. Figures 6.12-6.17 begin to present the data in a similar manner to the BSRDE. Specifically, in (e), the peak pressure is plotted against interruption time ratio, comparable to Figure 6.4 , and in (f,g) the dimensionless pressure and time are plotted in a scatter plot, comparable to Figure 6.5, and contour plot, respectively. 
Figures 6.12 and 6.13 relate to Figures 6.6 and 6.7. These figures (e-g) show the reduced data conclusions. The variance of the system is apparent in these plots as well, indicative of the separate clusters seen in the contour plot. The lower equivalence ratio yields a more stable and predictable system which agrees with the findings in Section 6.2.1.

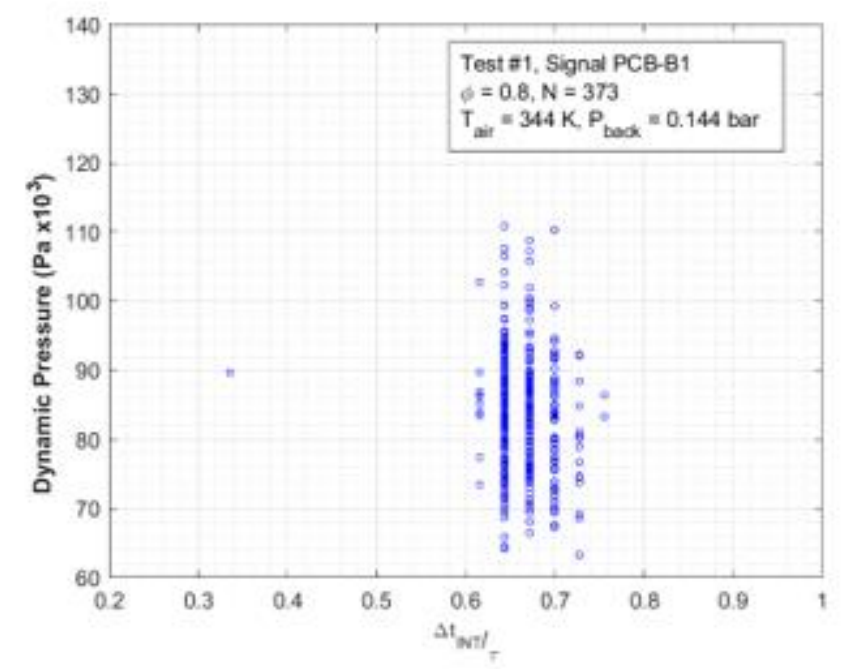

(e) Peak Pressure versus Interruption Time Ratio

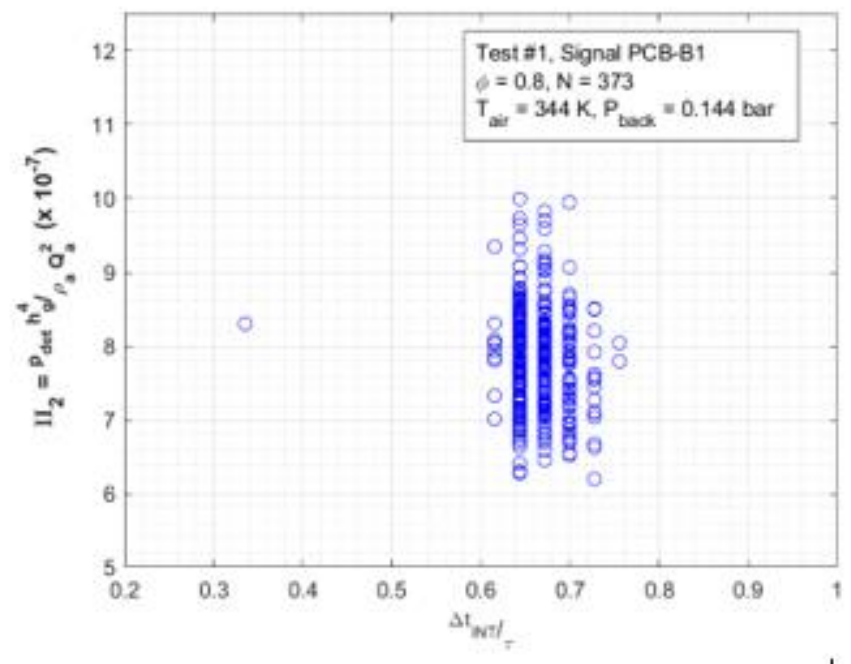

(f) $\Pi_{2}$ versus Interruption Time Ratio 


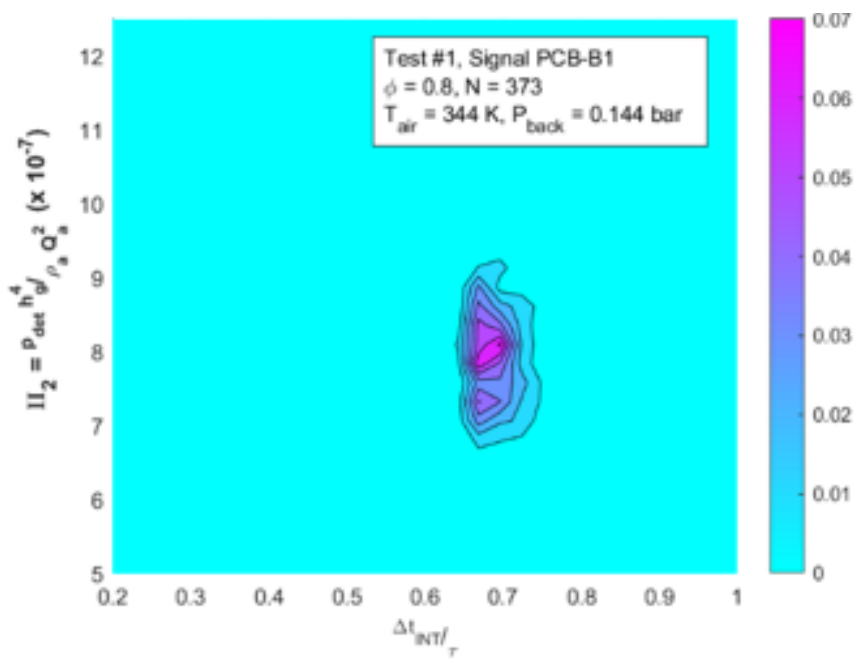

(g) Contour Plot of (f)

Figure 6.12: $Q=40,000 \mathrm{SCFH}, \phi=0.8, T=350 \mathrm{~K}, P=1.1 \mathrm{~atm}$

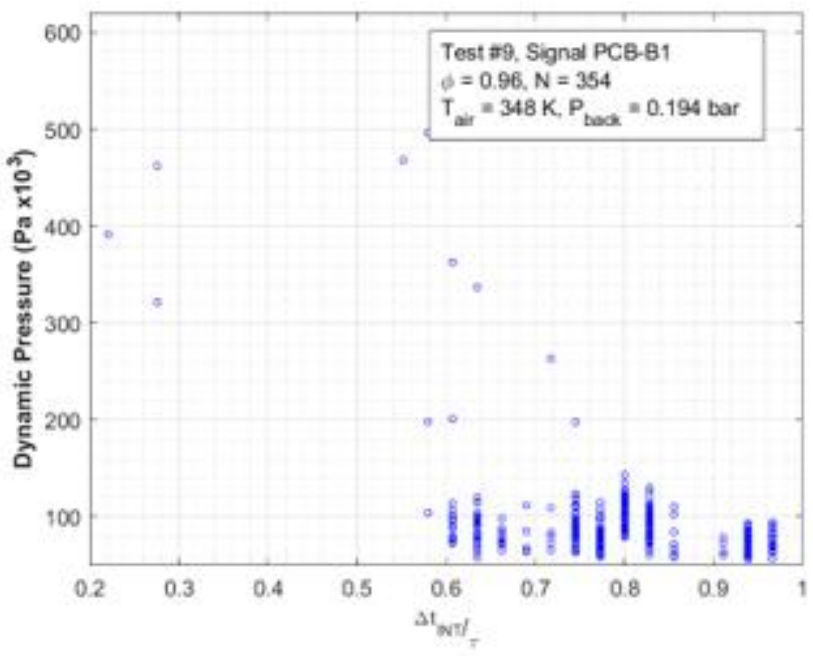

(e) Peak Pressure versus Interruption Time Ratio

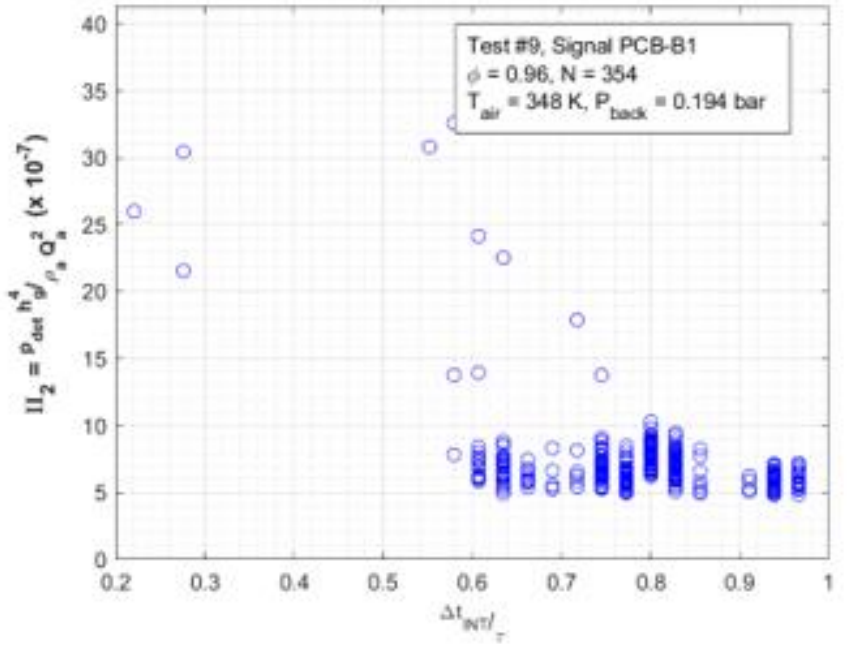

(f) $\Pi_{2}$ versus Interruption Time Ratio 


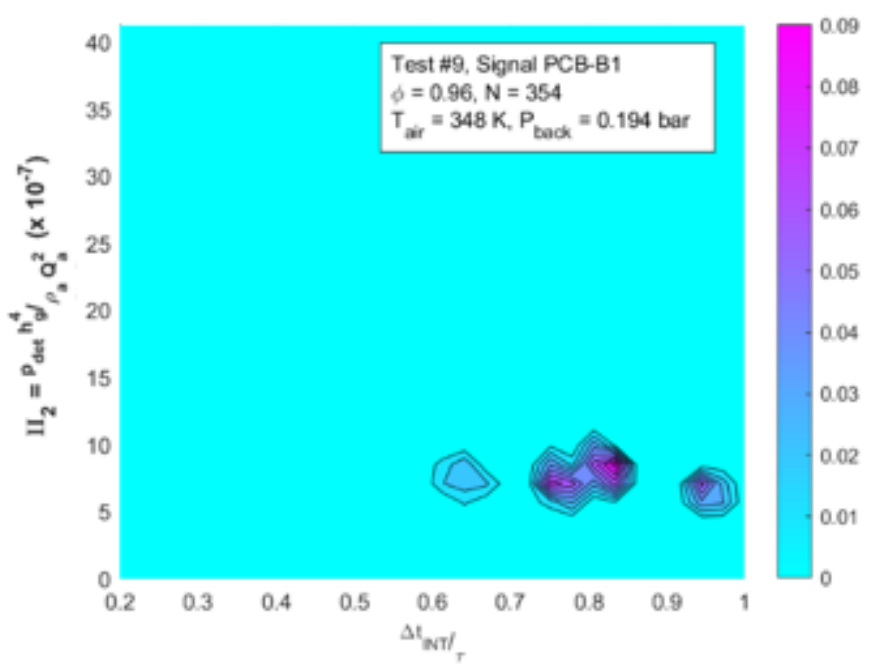

(g) Contour Plot of (f)

Figure 6.13: $Q=40,000 \mathrm{SCFH}, \phi=1.0, \mathrm{~T}=400 \mathrm{~K}, P=1.1 \mathrm{~atm}$

Figures 6.14 and 6.15 relate to Figures 6.8 and 6.9. Under these higher temperature conditions, the system appears to be less repeatable, especially in the $\phi=0.8$ case. The two groupings suggest mode switching or changes in stability through the duration of the test. This distinct grouping of data could also be due to the two, co-rotating waves of different strength, as discussed in Section 6.2.1. However, the spectrogram analysis in Figures 6.8 and 6.9(a) does not agree with the co-rotating wave conclusion. It is interesting to note the large interruption time ratios in Figure 6.14. This would suggest that the interruption time defined in this way may not be indicative of RDE operational ability. With interruption ratios nearing 1.0, logic would suggest that the system would be struggling to sustain itself, however, reflecting on Figure 6.8 (a) and (c), that does not appear to be the case. 


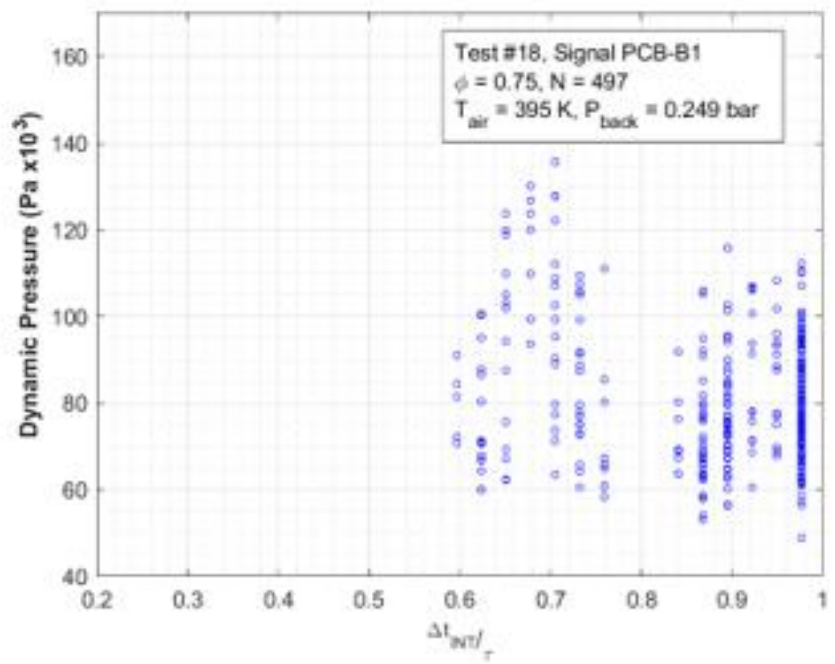

(e) Peak Pressure versus Interruption Time Ratio

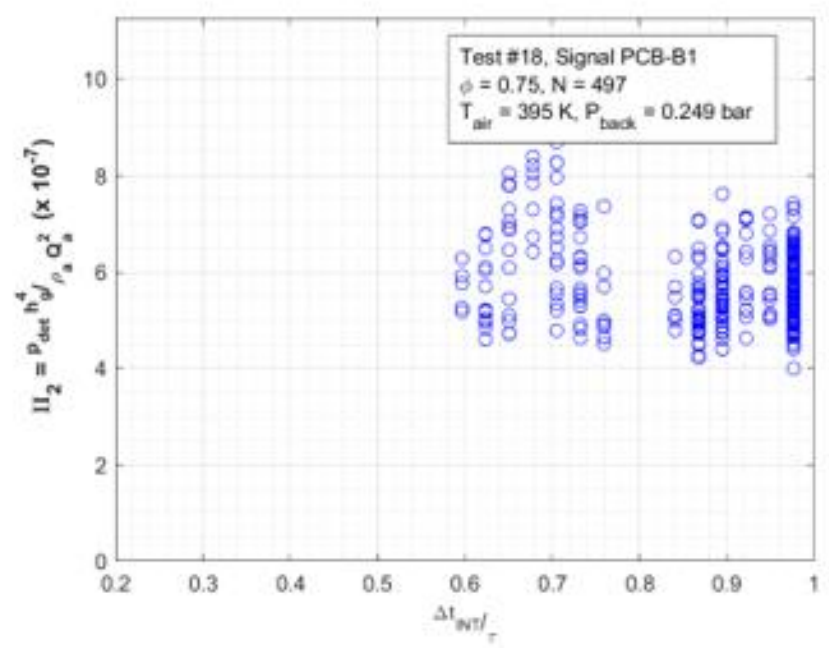

(f) $\Pi_{2}$ versus Interruption Time Ratio

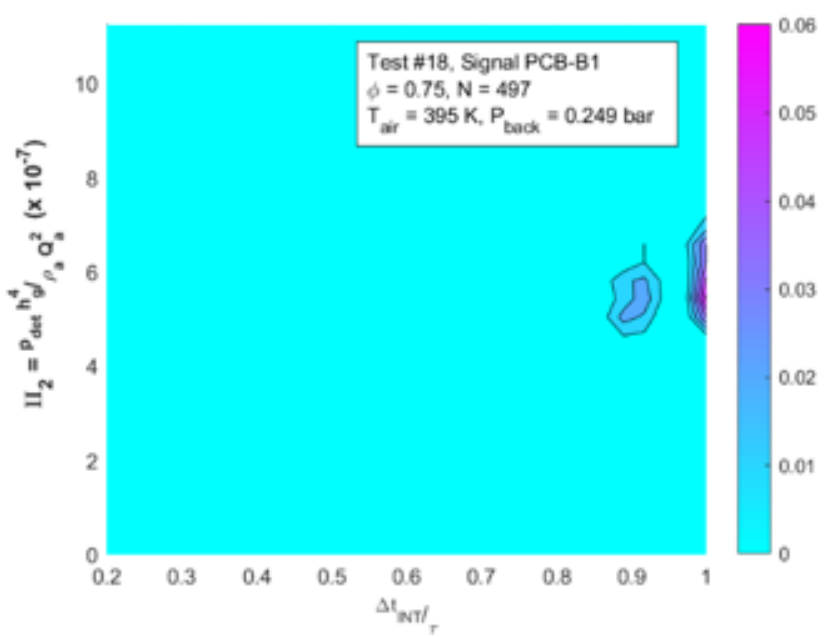

(g) Contour Plot of (f)

Figure 6.14: $Q=40,000 \mathrm{SCFH}, \phi=0.8, T=400 \mathrm{~K}, P=1.1 \mathrm{~atm}$ 


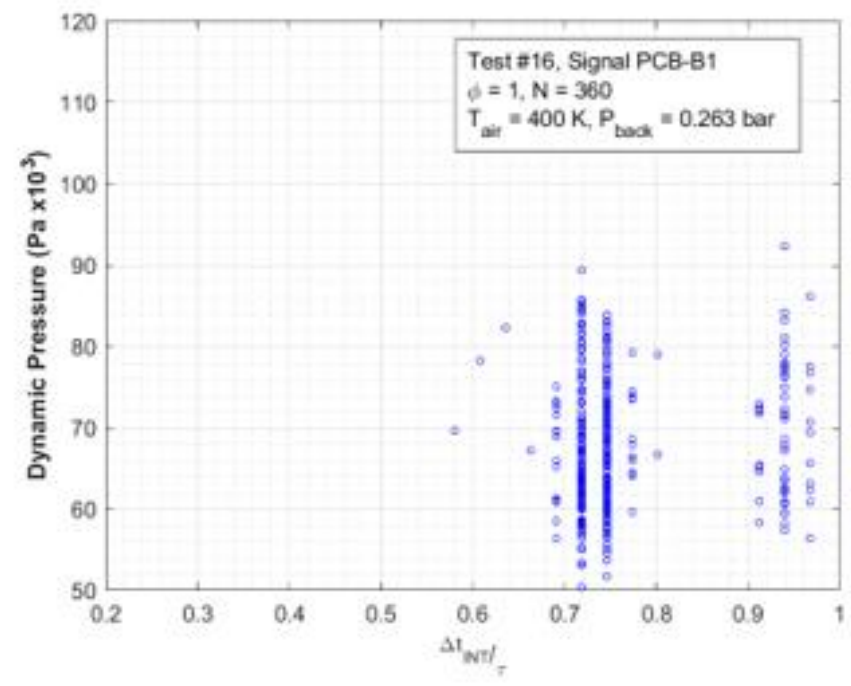

(e) Peak Pressure versus Interruption Time Ratio

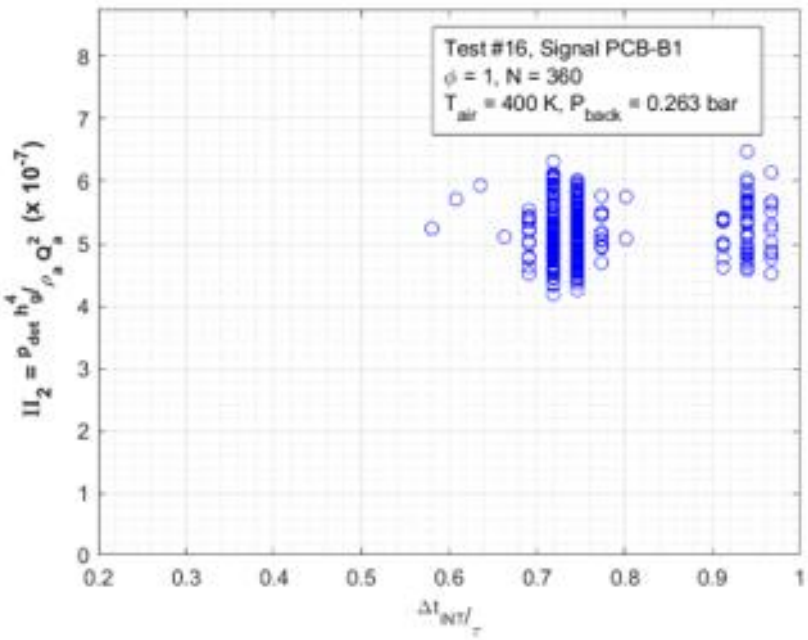

(f) $\Pi_{2}$ versus Interruption Time Ratio

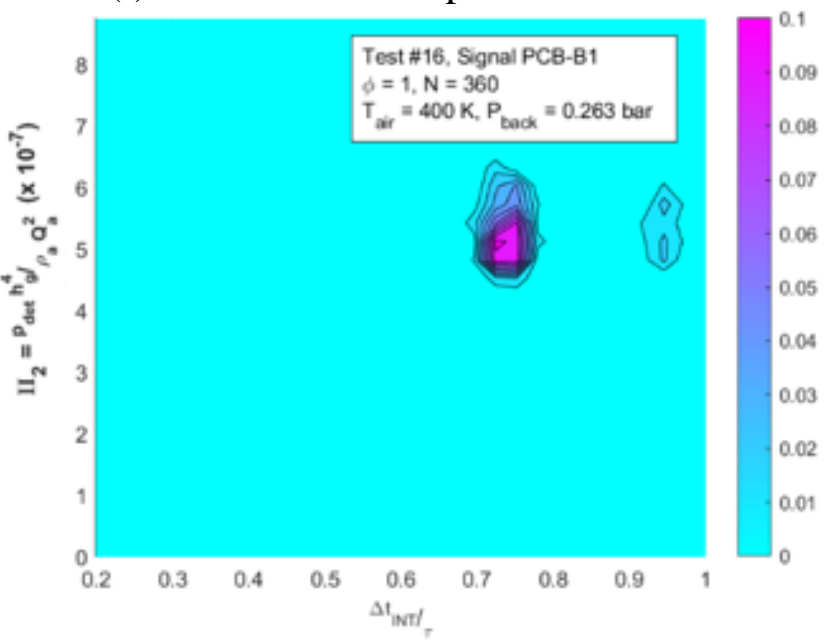

(g) Contour Plot of (f)

Figure 6.15: $Q=40,000 \mathrm{SCFH}, \phi=1.0, T=400 \mathrm{~K}, P=1.1 \mathrm{~atm}$ 
Figures 6.16 and 6.17 agree well with the preliminary findings in Figures 6.10 and 6.11. The strong variance within the RDE system at these conditions appears in (e-g) here as well. The higher and lower data groupings throughout the figures further indicates that there are two waves of uneven strength. Each grouping likely correspond to one of the two waves indicated in the spectrograms of Figures 6.10 and 6.11(a), thus the perceived variability from Figure 6.16 and 6.17 may not exist in reality. It is observed that at higher equivalence ratios, system variance is slightly improved comparatively.

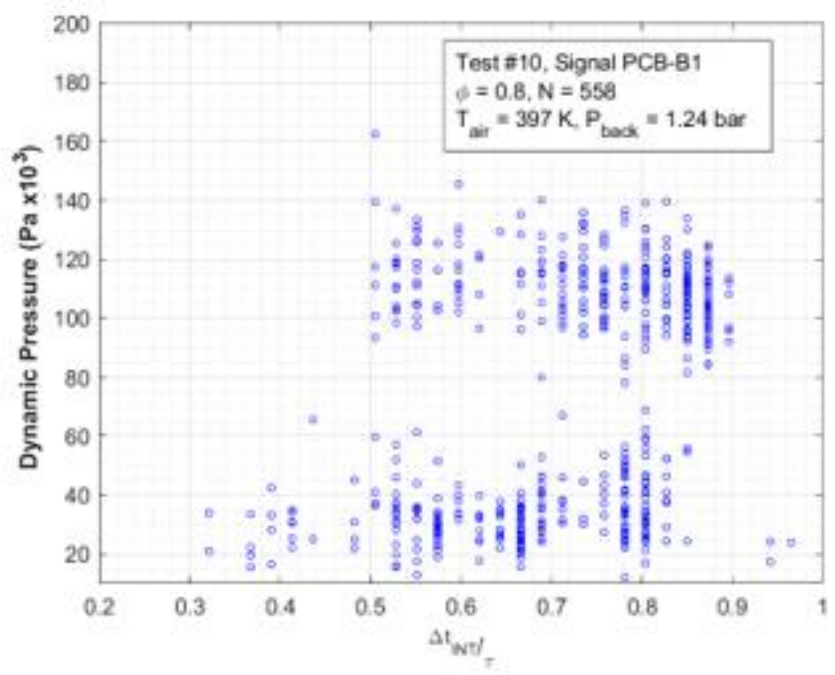

(e) Peak Pressure versus Interruption Time Ratio

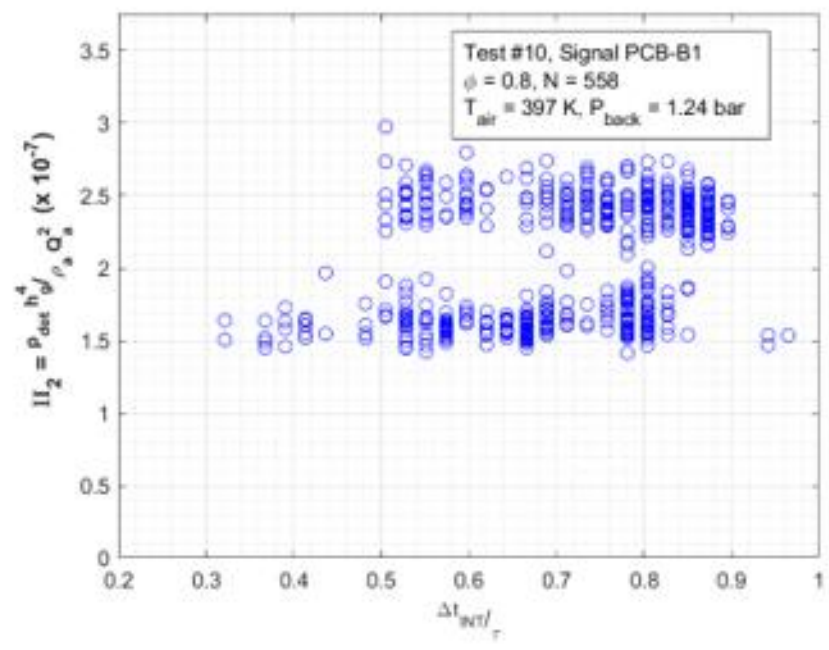

(f) $\Pi_{2}$ versus Interruption Time Ratio 


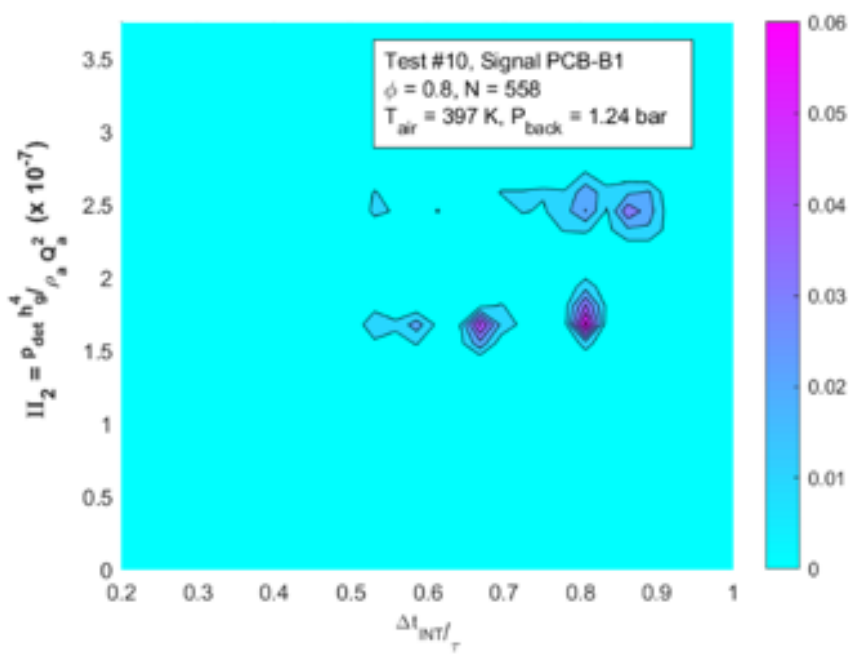

(g) Contour Plot of (f)

Figure 6.16: $Q=40,000 \mathrm{SCFH}, \phi=0.8, T=250^{\circ} \mathrm{F}, P=2.0 \mathrm{~atm}$

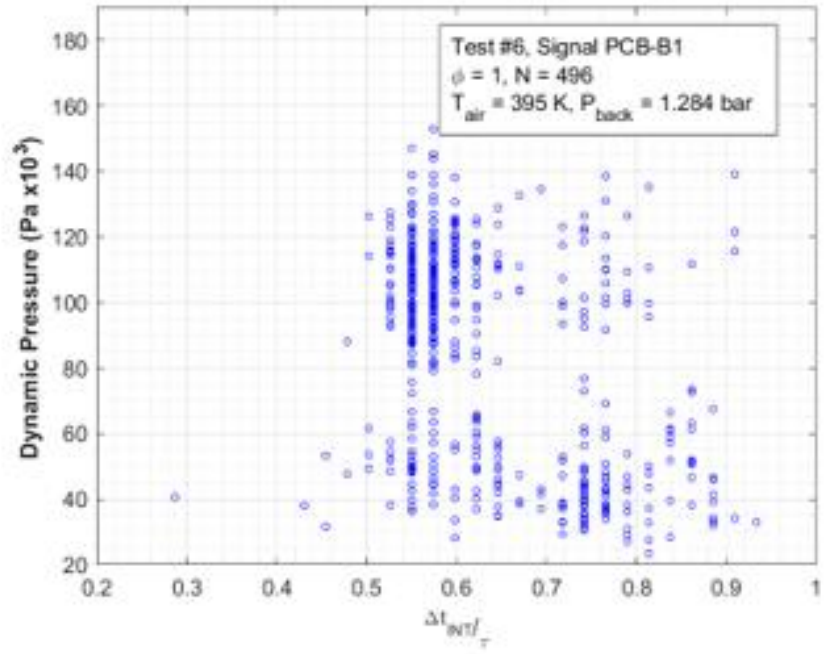

(e) Peak Pressure versus Interruption Time Ratio

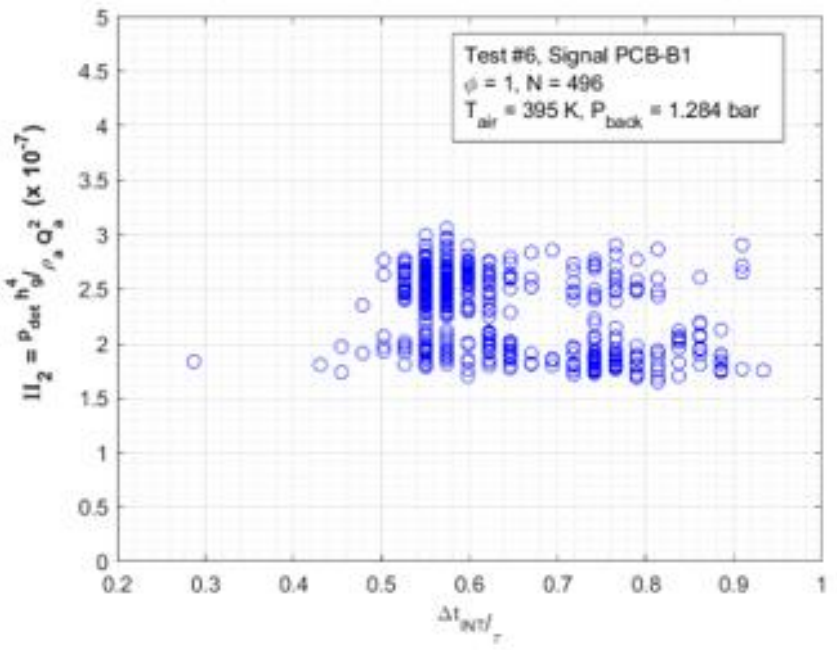

(f) $\Pi_{2}$ versus Interruption Time Ratio 


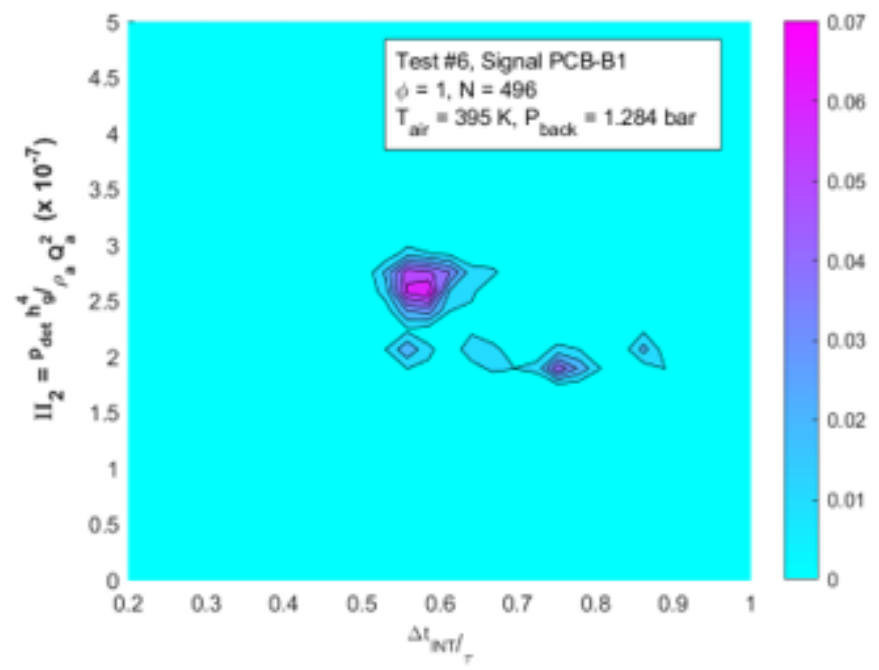

(g) Contour Plot of (f)

Figure 6.17: $Q=40,000 \mathrm{SCFH}, \phi=1.0, T=400 \mathrm{~K}, P=2.0 \mathrm{~atm}$

\subsubsection{Analysis Summary}

In order to summarize the performance of the LSRDE system within a set of testing conditions and relative to variations in testing conditions, the wave shape and dimensionless parameters are compared. First, the detonation wave shapes are presented for each set of testing conditions in Figure 6.18.

By comparing images from left to right, it can be seen that the higher equivalence ratio cases $(\phi=1.0)$ appear to have larger peak pressures relative to the lower equivalence ratio cases $(\phi=0.8)$ under the same testing conditions. This pattern would suggest that the reactants are more completely detonated in stoichiometric mixtures. The slower rise time in the lower equivalence ratio cases also suggests that the detonation occurring in the system is further from the theoretical limit of the von Neumann spike. This finding works to support the conclusion that at lesser equivalence ratios, incomplete detonation occurs. 
Additionally, observe by comparing cases vertically, increased air pre-heat temperature appears to result in slightly lower peak pressures. This could be similar to the relationships between upstream heating, mode switching as discussed in Section 1.6.

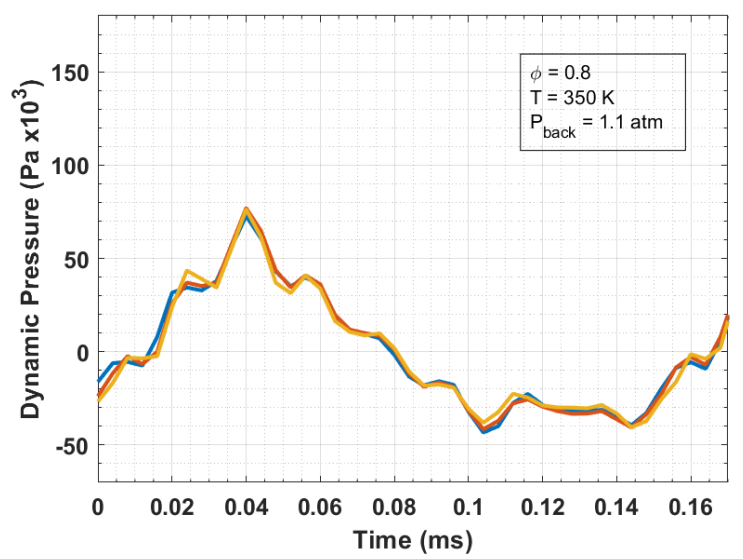

(h) $\phi=0.8, \mathrm{~T}=350 \mathrm{~K}, \mathrm{P}=1.1 \mathrm{~atm}$

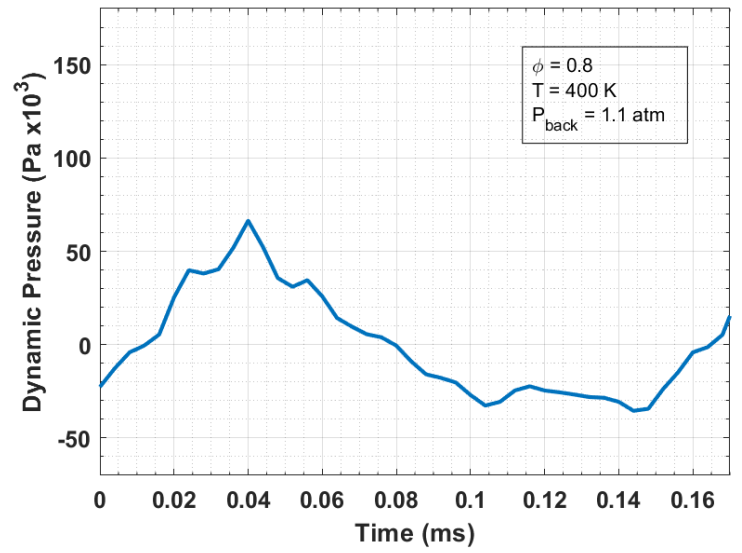

(j) $\phi=0.8, \mathrm{~T}=400 \mathrm{~K}, \mathrm{P}=1.1 \mathrm{~atm}$

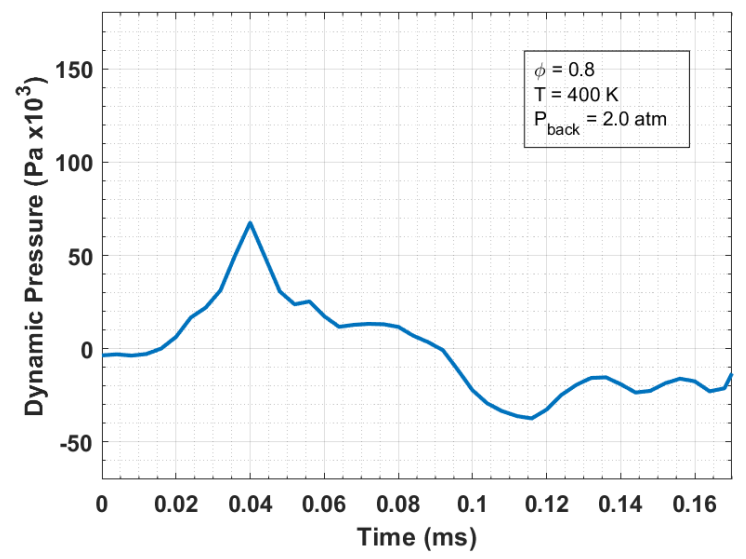

(l) $\phi=0.8, \mathrm{~T}=400 \mathrm{~K}, \mathrm{P}=2.0 \mathrm{~atm}$

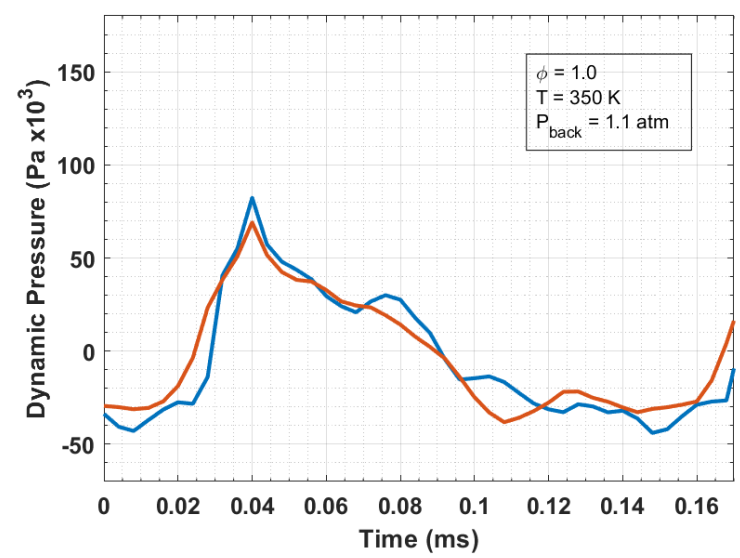

(i) $\phi=1.0, \mathrm{~T}=350 \mathrm{~K}, \mathrm{P}=1.1 \mathrm{~atm}$

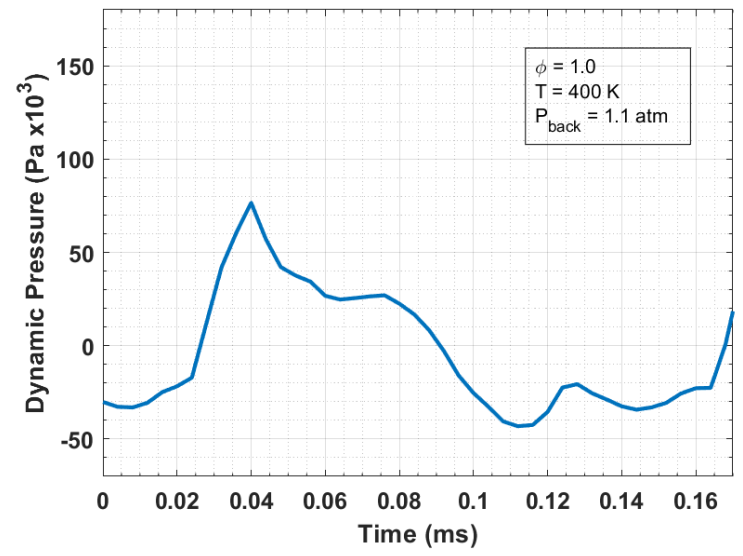

(k) $\phi=1.0, \mathrm{~T}=400 \mathrm{~K}, \mathrm{P}=1.1 \mathrm{~atm}$

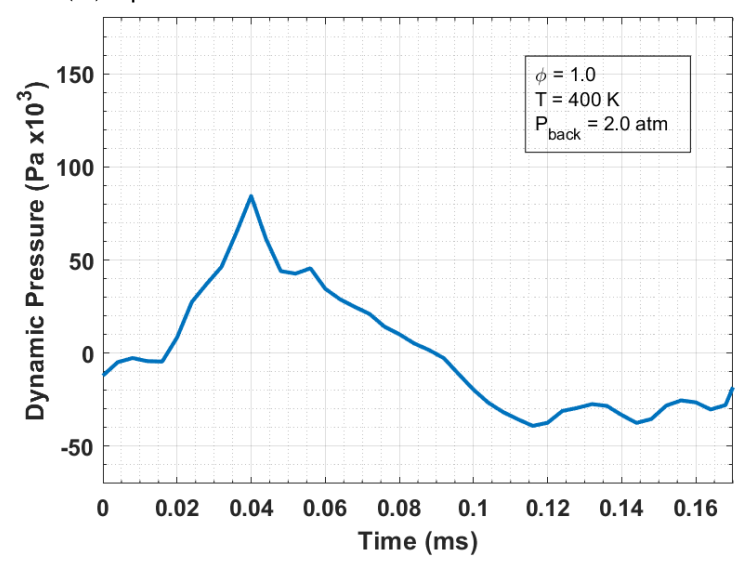

(m) $\phi=1.0, \mathrm{~T}=400 \mathrm{~K}, \mathrm{P}=2.0 \mathrm{~atm}$

Figure 6.18: Average Wave Forms plotted together by test conditions 
Unfortunately, the data did not allow for a comparison of nominal pre-heat temperature and back pressure to an increased back pressure case. Thus, no conclusions can be drawn in regards to the effect of elevated back pressure independent of the pre-heat temperature.

Furthering the analysis from Figure 6.18, Figure 6.19 presents each averaged wave form on the same axes. There is not enough discernible difference in these signals to identify many concrete trends.

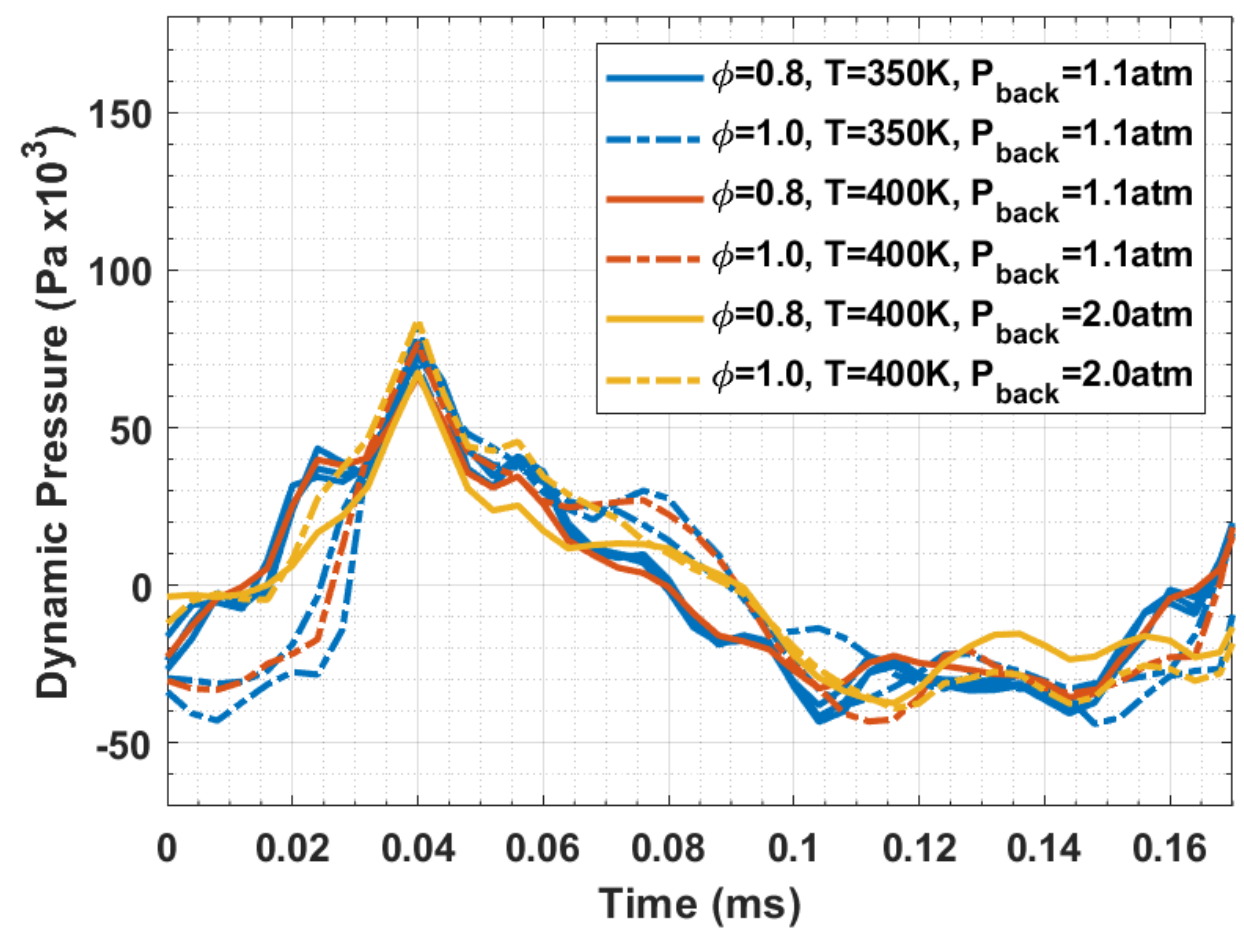

Figure 6.19: Average Wave Forms plotted together

Although the LSRDE system variation, which has previously been discussed in Section 5.2, restricts a clean comparison with the BSRDE, an attempt is made in Figure 6.20. With such a large number of sample points, it is impossible to see any trends. Thus, an average is calculated for each set of testing conditions. 


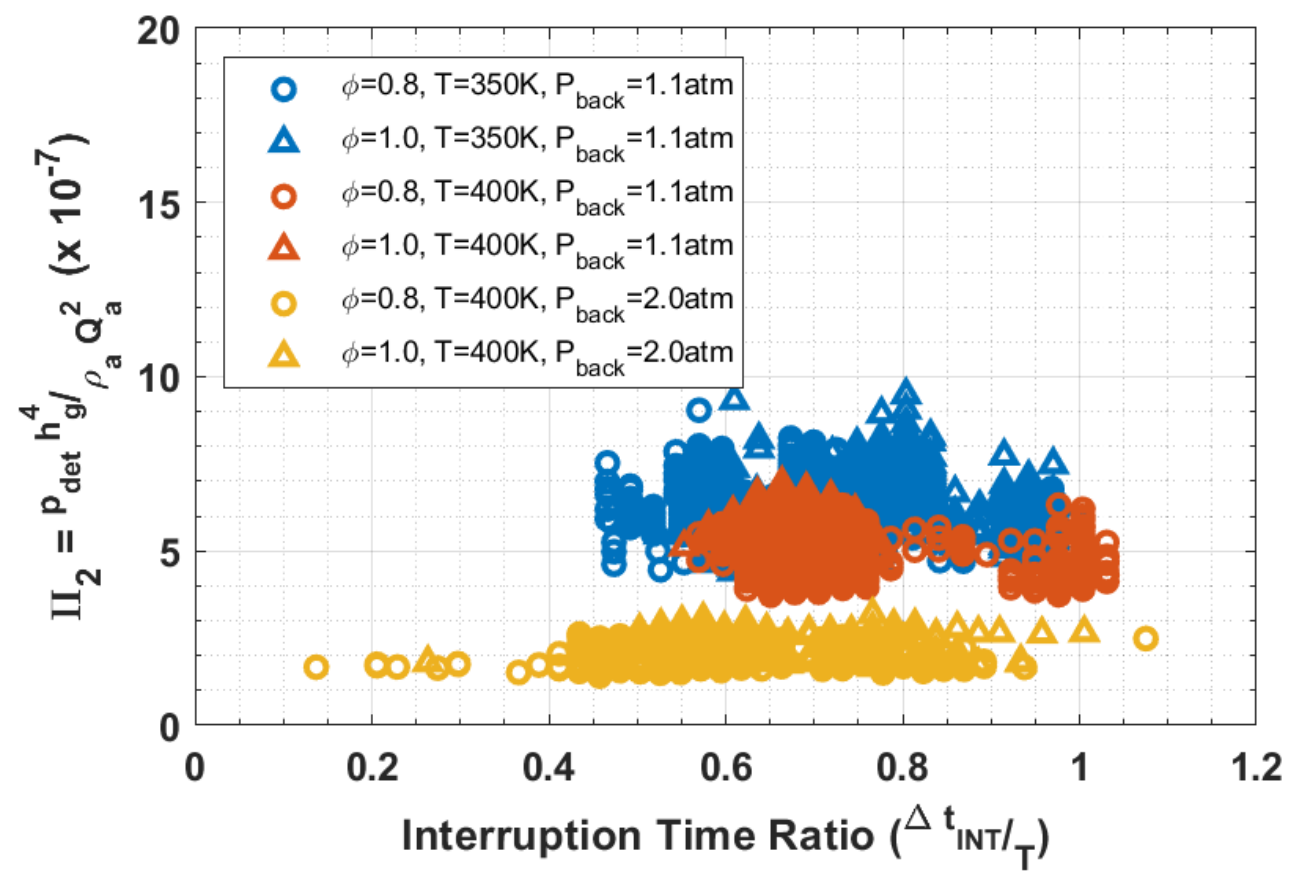

Figure 6.20: $\Pi_{2}$ versus interruption time ratio for all tests

Figure 6.21 further indicates the influence of system variance on drawing conclusions from these data reductions by introducing error bars on the averaged data. This plot does suggest that at the higher air supply pre-heat temperature and exhaust back pressure, the system operates at a significantly reduced dimensionless pressure term and interruption time ratio. This could be a significant finding due to the reality that an RDC would be subject to very high pressure when integrated into a gas turbine engine, however, any speculation or conclusions must be taken in consideration of the system variation, uncertainty, and the accompanying doubt in the trends of these results. If the RDE environment could be more deeply understood in order to select a steady operating time window, the variance would ideally decrease, and these trends may prove to be real. 


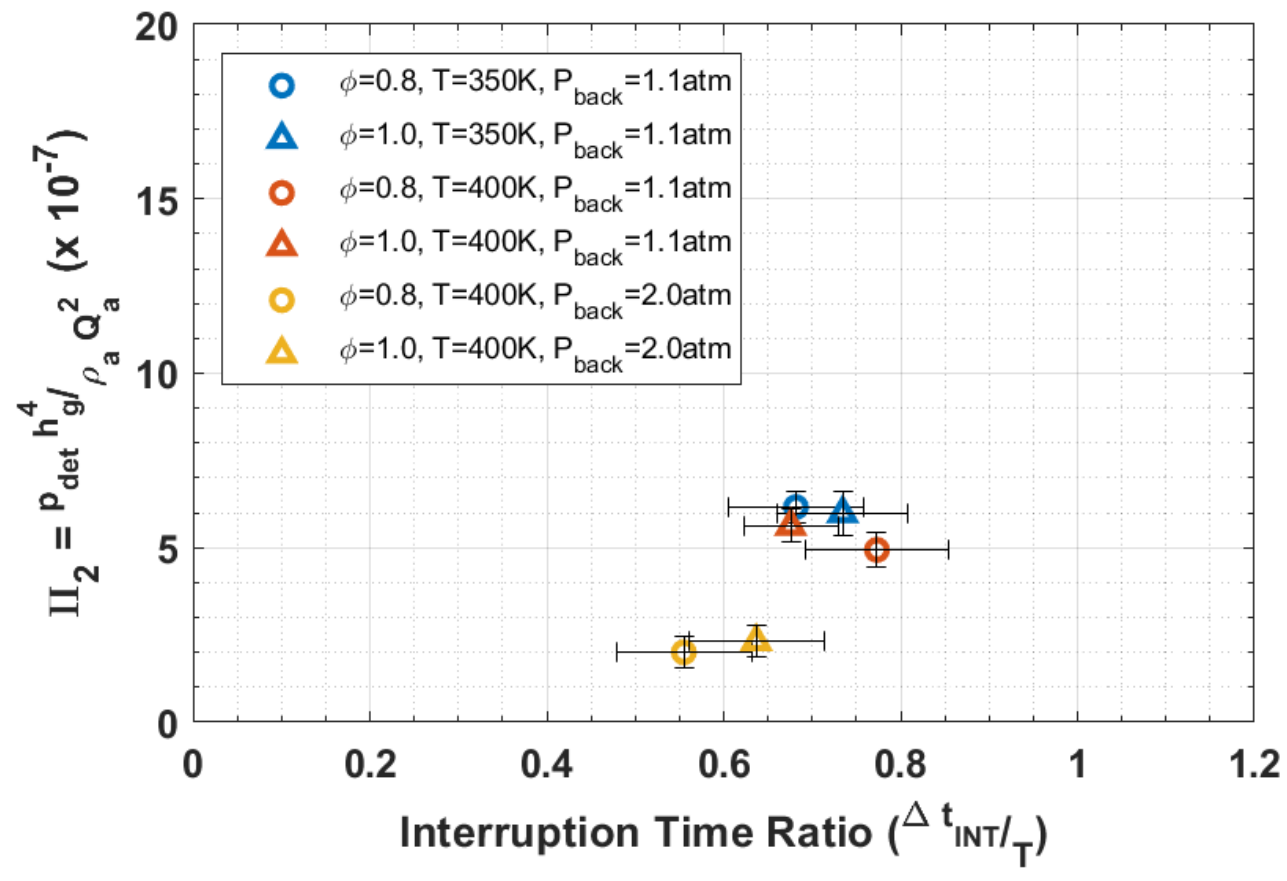

Figure 6.21: Averaged $\Pi_{2}$ versus interruption time ratio for all tests with uncertainty 


\section{Conclusions}

An investigation into the scaling laws defining a rotating detonation engine (RDE) has been conducted in tandem with an experimental comparison between two experimental RDE facilities at the U.S. Department of Energy's (DOE) National Energy Technology Laboratory (NETL). The first facility is a 6-inch diameter lab scale rotating detonation engine, and the second is a linearized bench scale RDE. The LSRDE is utilized to investigate the performance of an RDC under varying testing conditions such as equivalence ratio, air supply pre-heat temperature, and exhaust back pressure. The BSRDE enables optical access to the plenums of the linearized RDE which proves to be useful in observing the dynamic relationship between the detonation channel and supply plenums using Schlieren Imaging techniques. Accomplishments of this work include:

- Preliminary dimensional analysis on the RDE environment and thorough investigation into two dimensionless parameters defining RDE operation was completed.

- MATLAB data reduction codes were created to automate data analysis in both experimental facilities at NETL.

- Methods for improvement of the two experimental facilities in order to facilitate a more direct comparison and decrease the measurement uncertainty were proposed and detailed.

- Preliminary investigation into defining RDE operational stability via a MATLAB code was performed and direction for succeeding analyses was suggested.

The dimensional analysis resulted in a dimensionless pressure and time term. Dimensionless time (interruption time ratio) was analyzed as a function of non-dimensional peak pressure $\left(\Pi_{2}\right)$. In $\Pi_{2}$, peak channel pressure is non-dimensionalised by a combination of the air injection gap height, air supply density, and air supply flow rate. Mathematically, $\Pi_{2}$ encompasses a fluid dynamic detonation pressure non-dimensionalised by the air supply momentum flux and a geometric 
parameter. The interruption time ratio consists of the interruption time, defined as the time during which the dynamic pressure is excited above steady state due to a detonation event, and the wave period determined by the peak frequency of the FFT of the signal in the LSRDE or measured experimentally in the BSRDE.

In the BSRDE, there exist weak correlations between the dimensionless pressure term and the interruption time ratio, depicted in Figure 6.5. This relationship suggests that both interruption time ratio and non-dimensional peak pressure decrease with increasing air supply flow rate due to the increased static pressure in the air plenum. Physically, this indicates that the higher air supply flow rates effectively push the combustion products and shock reflections out of the plenum faster compared to lower flow rates. As depicted in Figure 6.3, the peak channel pressure does not vary across changing flow rate conditions, thus the non-dimensional peak pressure decreases directly from the incorporation of the air flow rate into it's determination. These trends are useful in understanding the performance of the BSRDE, but it is unclear how these findings relate to the LSRDE due to the differences in ignition and flow mechanisms between the two facilities. A design consideration in the BSRDE was to decouple the detonation wave from the supply flow rates. This was accomplished by establishing a detonation wave in a pre-detonator tube, upstream of the test section, which incorporated a split-path to simulate an RDE period. The detonation expands out of the pre-detonator tube into a linearized RDE with a non-reacting fuel surrogate and air being supplied through the plenums. Thus, the volume flow rate of air has no interaction with the strength of the detonation expanding into the channel. In the LSRDE, the increased air supply flow rate is strongly tied to the intensity of the detonation event. Increased air supply impacts mixedness and local equivalence ratio, both of which have been shown to impact detonation strength in the form of mode-switching and wave bifurcation $[15,16]$. It may be that increased 
supply flow rates increase mixing and therefore increase detonation strength by inducing more complete detonation, which in turn generates more backflow. It could also be that at elevated supply flow rates, the system bifurcates into multi-wave systems, lowering the peak pressures and reducing the backflow as a result of increasing the relative operating frequency relative to the transducer location and reducing the time permitted for the inlet to recover. The important takeaway is that there are far too many additional considerations and complications that exist within the LSRDE for any useful relationships to be drawn between the two facilities under this investigation.

It was desired to attempt to extrapolate a recovery time in the LSRDE by correlating $\Pi_{2}$ and interruption time ratio to recovery time in the BSRDE. Recovery time was previously studied using the BSRDE at various geometric conditions by Bedick, et al. and Sisler [26,28]. Unfortunately, not enough data existed to meaningfully attempt this correlation in this work. Additionally, the relationships presented between non-dimensional peak pressure and interruption time ratio indicate that any correlation found in the BSRDE cannot be extrapolated to the LSRDE. This is due to the drastic difference in flow rates and detonation mechanisms. The non-dimensional peak pressure parameters are four orders of magnitude different between the two facilities. Interruption time ratio is also significantly higher in the LSRDE comparatively. In fact, the minimum interruption time ratio determined in the LSRDE is above the maximum of that observed in the BSRDE. This could be, at least in part, due to the method through which the RDE period is estimated in the BSRDE.

Within the LSRDE, conclusions must be formulated in consideration of the large natural variability within the system. The dimensionless plot, Figure 6.21, does indicate that the LSRDE operates at a lower non-dimensional peak pressure and interruption time ratio mode at increased pre-heat 
temperature and pressure. The separation of these data points does overcome influence of system variability, thus the conclusion can be taken with reasonable confidence, though further investigation is needed as this study incorporates a limited number of data sets. This finding is encouraging for succeeding work using this methodology due to the reality that an RDC employed in a traditional gas turbine environment would be exposed to significant back pressure due to turbine integration and upstream heating.

Comparing averaged wave forms among various testing conditions indicates that stoichiometric conditions induce more complete detonation to occur relative to lesser equivalence ratio conditions. This is indicated by the shape of the von Neumann spike in each of the plots in Figure 6.18. The slower rise time of the $\phi=0.8$ cases indicates larger deviation, relative to the $\phi=1.0$ cases, from the theoretical limits and predictions given by Zeldovich-von Neumann-Doring (ZND) theory.

\section{Recommendations}

\subsection{BSRDE Fill Time}

In the linear rig, the detonation is driven by the pre-detonator tube. The air and fuel supply to the channel via the plenums is purely a placeholder, having no chemical-kinetic relationship with the detonation wave. One potential source of unrepeatability in the BSRDE is the pre-detonator ignition. No timing mechanism currently exists which controls the ignition timing. It is presumed that the pre-detonator tube fills for a long enough time to fully fill itself and pour into the channel. This also impacts the two-wave simulated RDE period. The additional 37-inch loop for the second wave is filled with reactants and is likely the reason that such drastic variation in first and second wave peaks was observed during data analysis. The pre-detonator tube in the BSRDE is 30 inches 
in length from the ignition source to the split path fitting, $1 / 4$ " in outer diameter, and 0.035 inches in thickness, with supply flow rates of 2 SLPM $\mathrm{H}_{2}$ and 3 SLPM air.

$$
\begin{gathered}
\forall_{\text {PDtube }}=\pi\left[\frac{d_{\text {tube }}-2\left(t_{\text {tube }}\right)}{2}\right] L_{\text {tube }}=1.25 \cdot 10^{-5} \mathrm{~m}^{3} \\
t_{\text {fill }}=\frac{\forall_{\text {tube }}}{\dot{\forall}_{\text {combined }}}=\frac{\forall_{\text {tube }}}{\dot{\forall}_{H_{2}}+\dot{\forall}_{\text {air }}}=0.15 \mathrm{~s}
\end{gathered}
$$

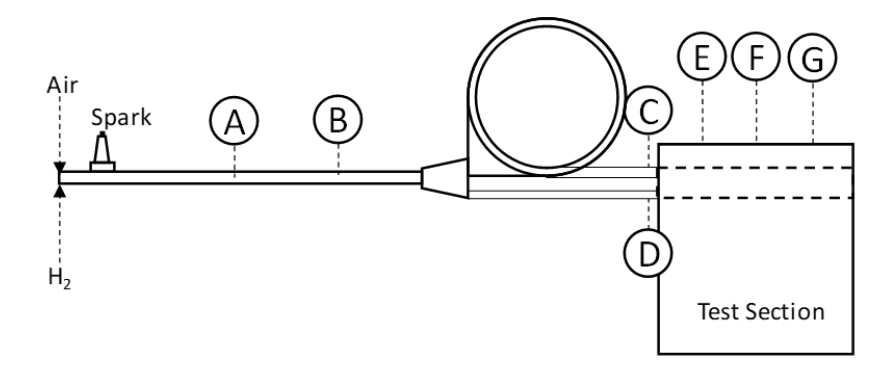

Figure 8.1: BSRDE Pre-detonation Tube Schematic

During the firing sequence, the BSRDE pre-detonator flow controllers are allowed to reach a steady operating point, which takes much longer than the 0.15 seconds that are theoretically needed to overfill the tube. This ensures that there are reactants within the channel for each test. To eliminate any possible variation in, and within, tests due to the fill time of the pre-detonator, the CJ velocity could be used to predict the time it takes the detonation wave to travel to the split path fitting. This could then be incorporated into the LabView control routine to time the pre-detonator fueling and spark ignition in a way that allows the reactants to just barely fill the tube before the detonation wave splits. For the BSRDE, the pre-detonator is set to generate a shock wave at an equivalence ratio of 1.2 at atmospheric temperature and pressure. Thus the CJ velocity, using the Shock and Detonation Toolbox [23], is $1967.8 \mathrm{~m} / \mathrm{s}$. This calculation is idealized and does not consider mixing and frictional losses, yielding an overestimate of wave speed. The calculation below shows that incorporating wave speed into the determination of ignition timing is likely 
insignificant relative to the fill time. The point of this analysis is simply to increase repeatability by eliminating inconsistencies in testing.

$$
t_{\text {fire }}=t_{f i l l}-\frac{L_{t u b e}}{U_{C J}}=0.1496 \mathrm{~s}
$$

\subsection{LSRDE Measurement Techniques}

There are two measurement methods that could be addressed to bolster this analysis. The first is using cooled dynamic pressure transducers and the second is calculating wave speed from flow imaging.

\subsubsection{Cooled Dynamic Pressure Transducers}

As discussed in Section 2.3, significant measurement discrepancy and uncertainty arises through the use of ITP mounted PCBs to capture detonation events. With attenuation of up to 54\% and signal delay of $\sim 6 \mu$ s, there is significant room for improvement. Recent work by Naples, et al. [40] indicates that the ITP configuration is largely to blame for any measurement discrepancy. Using water-cooled PCBs in a flush-mounted configuration would greatly increase the accuracy in which the detonation event is being measured.

\subsubsection{Wave Speed via Image Processing}

Bluemner, et al. have utilized an aft aligned high speed camera to capture the luminosity of the detonation wave in real time [41]. By manipulating this data using image processing techniques, they are able to get more realistic and time-varying wave velocity estimates. The study showed that for a single steady wave operation, the image based wave velocity can vary as much as 200 $\mathrm{m} / \mathrm{s}$ compared to the FFT based velocity, which has been used in most prior studies. Their technique is shown below in Figure 8.2. 


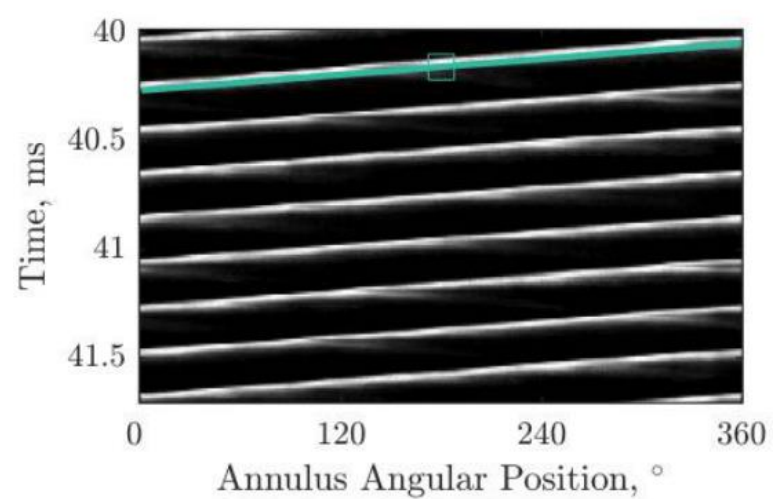

(a)

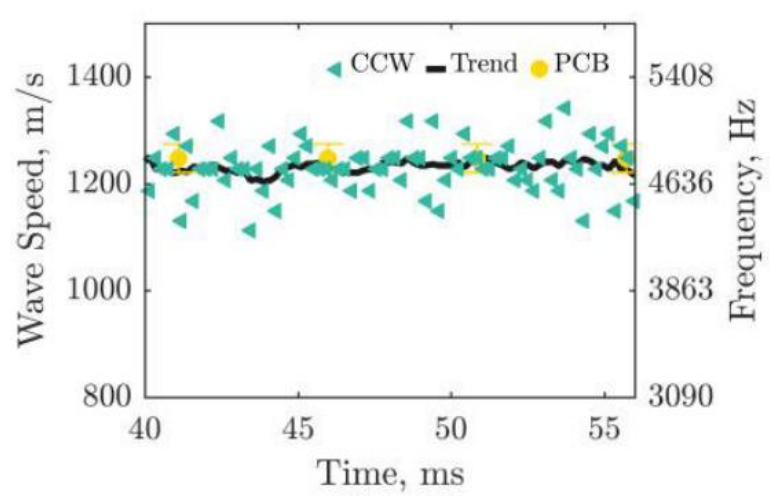

(b)

Figure 8.2: Radially averaged luminosity within the RDC annulus plotted over annulus angular extent and run time, together with a measured sample characteristic path line (a), showing stable one-wave operation. Detonation wave speed for a subset of laps, as calculated based on the luminosity characteristics, together with their moving average and the short-time

FFT of PCB $06(b)$. A good agreement of pressure and luminosity measurement results confirms the ability to also reveal lap-to-lap fluctuations in wave speed that are masked by the FFT. [41]

At NETL's LSRDE facility, optical access is available, oriented upstream from the exhaust plane.

Thus far, these images have been used to visually confirm the conclusions drawn from spectrogram plots, however, if a similar analysis was performed for the LSRDE facility, optically derived wave speed and PCB derived wave speed could be compared to determine a domain of data in which the RDC operates in a more repeatable and distinct mode. This would enable a more complete and conclusive analysis of the methodology presented in this study.

\subsection{LSRDE Configuration}

Similar to NETL's BSRDE, Fotia, et al. [42] had previously designed and tested an unwrapped, two-dimensional RDC. This experimental setup attempted to mimic a full RDC cycle by intiating two consecutive detonation waves through the linearized channel. After testing was completed, it was found that a deflagration region attached to the linear channel and caused significant damage to the transparent windows, limiting the use of a simulated RDC period in addition to the re-use of the facility. However, a few interesting observations came of this study. Firstly, via Schlieren Imaging, the induced pressure wave within the fuel plenum was found to be $64 \%$ of the detonation 
wave speed in the channel. This suggests that after several cycles, there will be a secondary interaction between the detonation wave, previous fuel plenum pressure wave, and successive fuel plenum pressure wave. These interactions may be influencing the consistency of mixedness in the channel, and therefore the RDC operating mode and stability.

Additionally, due to the deflagration within the channel, the thermal distribution through the channel is visible from the burn patterns on the polycarbonate windows.

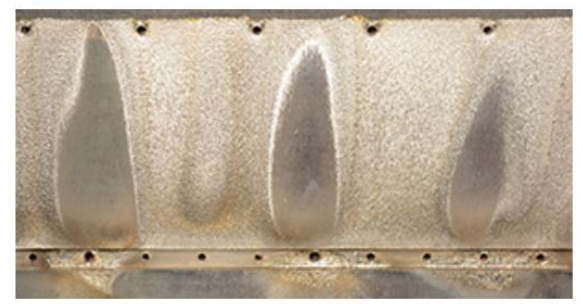

a) Channel wall opposed to air plenum slot

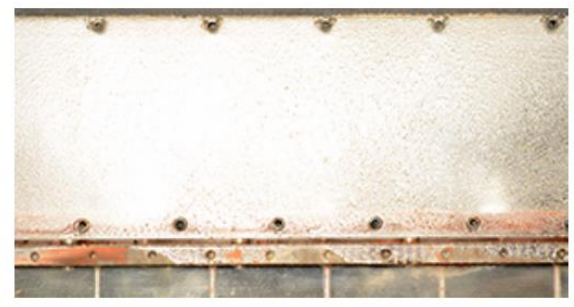

b) Channel wall above air plenum slot

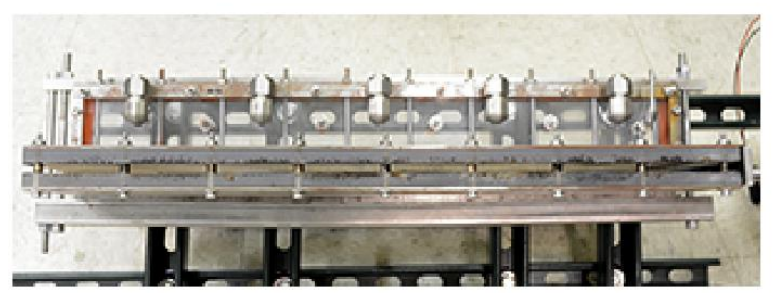

c) Location of air feed ports at plenum

Figure 8.3: Images of Experimental Rig after run, supporting argument for greater mixing at air plenum feed tubes.

Of particular interest are a) and c) in Figure 8.3. These images show that there is uneven composition within the channel corresponding to the air supply lines that feed the plenum. This phenomena could cause the low frequency instability seen in NETL's data. As seen in Figure 8.4, the NETL LSRDE fuel plenum is supplied by 6 feed lines. Though the injection configuration is undoubtedly different, such a large azimuthal gap in distribution could cause local variation in equivalence ratio, thereby affecting the strength and speed of the detonation wave. 


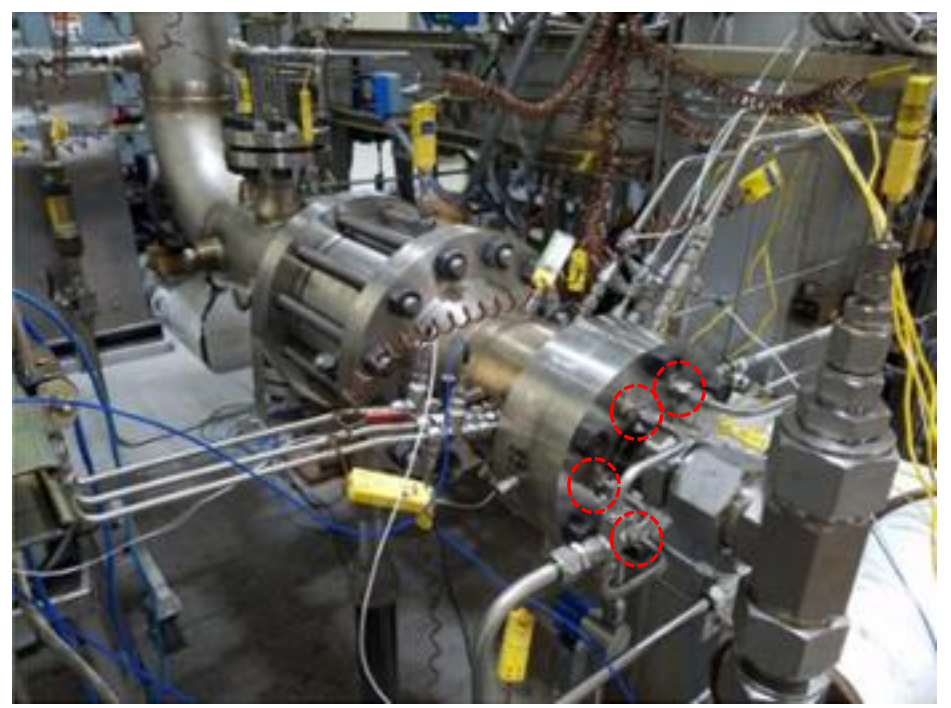

Figure 8.4: LSRDE Facility at NETL, depicting air plenum supply plumbing

\subsection{Dimensional Analysis}

The dimensional analysis was performed with the repeating variables of air density, air gap height, and air supply flow rate due to their design and control considerations in the RDE rigs. Had an alternative set of repeating variables been selected, the results would have differed considerably. Namely, it would be interesting and of value to perform a dimensional analysis using the speed of sound or the air injection velocity as a repeating variable. Additionally, succeeding iterations of this analysis should incorporate the fourth fundamental dimension of temperature, resulting in an aero-thermal analysis of the RDE environment.

\subsection{Characterization of LSRDE Detonation Strength}

Under current facility design, if representative detonation waves for certain sets of operating conditions within the LSRDE could be defined and consistently reproduced in the BSRDE, then real implications of geometric alterations could be studied at each flow condition. This would require correlating the pre-detonation tube fill conditions in the BSRDE to the detonation strengths observed within the LSRDE. The results of those studies would only be of value if the LSRDE system operability is better understood across various testing conditions. 


\subsection{Multi-wave Consideration in Data Reduction}

The code developed to reduce data in the LSRDE did not consider co-rotating waves of varying strength. Thus, in the summarizing figures, such as Figure 6.17(g), inconsistent system mode is suggested by the distinct groupings in the interruption time. Based on the spectrogram in Figure 6.11(a), a two wave, co-rotating mode is indicated. These findings suggest that the MATLAB code is successfully identifying the two waves of varying strength, but mis-representing them by only considering each wave in the system to be of similar strength. In effect, the wave stacking in Figure 6.11(c) and the dimensionless plot in Figure 6.17(f,g) demonstrate inconsistent operation, however in reality, the system is likely sustaining consistent two-wave operation as indicated by the spectrogram (Figure 6.11(a)). By incorporating this observation into succeeding analyses, system variation is likely to be substantially reduced. 


\section{References}

[1] Nordeen, C. A., Thermodynamics of a Rotating Detonation Engine.

[2] Giampaolo, T., 2009, Gas Turbine Handbook: Principles and Practice, Fairmont Press.

[3] Hunt, R. J., 2011, “The History of the Industrial Gas Turbine (Part 1 The First Fifty Years 1940-1990)," IDGTE.

[4] Saravanamuttoo, H. I. H., Rogers, G. F. C., Cohen, H., and Straznicky, P. V, 2009, Gas Turbine Theory, Pearson Prentice Hall, Harlow, England; New York.

[5] Lefebvre, A. H., and Ballal, D. R., 2010, Gas Turbine Combustion: Alternative Fuels and Emissions, Taylor \& Francis, Boca Raton, FL.

[6] University of Texas at Arlington, 2014, "Pulsed Detonation Engines" [Online]. Available: https://arc.uta.edu/research/pde.htm.

[7] Richards, G. A., 2012, New Developments in Combustion Technology - Part II: Step Change in Efficiency.

[8] Hayes, S. A., Nix, A. C., Nestor, C. M., Billups, D. T., and Haught, S. M., 2017, "Experimental Investigation of the Influence of Freestream Turbulence on an Anti-Vortex Film Cooling Hole," Exp. Therm. Fluid Sci., 81, pp. 314-326.

[9] Agency, E. P., 2019, "Sources of Greenhouse Gas Emissions" [Online]. Available: https://www.epa.gov/ghgemissions/sources-greenhouse-gas-emissions\%0A.

[10] U.S. Energy Information Administration, 2019, Annual Energy Outlook 2019, Washington, DC.

[11] Zel'dovich, Y., 1942, "On the Distribution of Pressure and Velocity of the Detonation 
Explosion Products, Particularly in Spherical Distribution of the Detonation Wave," J. Exp. Theor. Phys., 12(9), pp. 389-406.

[12] Nicholls, J. A., and Cullen, R. E., 1964, "The Feasibility of a Rotating Detonation Wave Rocket Motor: Final Report.”

[13] Voitsekhovskii, B. V., 1959, “Maintained Detonation,” Dokl. Akad. Nauk SSSR, 4(6), pp. 1207-1209.

[14] Frolov, S. M., Dubrovskii, A. V., and Ivanov, V. S., 2013, “Three-Dimensional Numerical Simulation of the Operation of a Rotating-Detonation Chamber with Separate Supply of Fuel and Oxidizer,” Russ. J. Phys. Chem. B, 7(1), pp. 35-43.

[15] Roy, A., Ferguson, D. H., Sidwell, T., O’Meara, B., Strakey, P., Bedick, C., and Sisler, A., 2017, “Experimental Study of Rotating Detonation Combustor Performance under Preheat and Back Pressure Operation," 55th AIAA Aerospace Sciences Meeting, pp. 1-16.

[16] Anand, V., St. George, A., Driscoll, R., and Gutmark, E., 2015, "Characterization of Instabilities in a Rotating Detonation Combustor,” Int. J. Hydrogen Energy, 40(46), pp. $16649-16659$.

[17] Driscoll, R. B., Anand, V., St. George, A. C., Stoddard, W., Munday, D. E., and Gutmark, E. J., 2015, "Numerical Investigation of Inlet Injection in a Rotating Detonation Engine," 53rd AIAA Aerosp. Sci. Meet., (January).

[18] Andrus, I. Q., Polanka, M. D., King, P. I., Schauer, F. R., and Hoke, J. L., 2017, "Experimentation of Premixed Rotating Detonation Engine Using Variable Slot Feed Plenum," J. Propuls. Power, 33(6), pp. 1448-1458. 
[19] Hariharan, A. R., and Babu, V., 2013, "Transverse Injection Into a Supersonic Cross Flow Through a Circular Injector With Chevrons,” J. Fluids Eng., 136(2), pp. 21204-21208.

[20] Bellini, R., 2010, "Ideal Cycle Analysis of a Regenerative Pulse Detonation Engine for Power Production," The University of Texas at Arlington.

[21] Braun, E. M., 2012, "New Detonation Concepts for Propulsion and Power Generation," The University of Texas at Arlington.

[22] Kirk, D. R., "MAE 5310: Combustion Fundamentals."

[23] Shepherd, J. E., 2019, "Shock and Detonation Toolbox" [Online]. Available: http://shepherd.caltech.edu/EDL/PublicResources/sdt/.

[24] Edwards, D. H., Thomas, G. O., and Nettleton, M. A., "Diffraction of a Planar Detonation in Various Fuel-Oxygen Mixtures at an Area Change," Gasdynamics of Detonations and Explosions, pp. 341-357.

[25] Roy, A., Ferguson, D., Sidwell, T., and Strakey, P., 2018, "Characteristics of a NonPremixed Rotating Detonation Combustor Using Natural Gas- Hydrogen Blend at Elevated Air-Preheat Temperature and Backpressure,” pp. 1-19.

[26] Bedick, C., Sisler, A., Ferguson, D., Strakey, P., Nix, A., and Billips, D., 2017, "Development of a Lab-Scale Experimental Testing Platform for Rotating Detonation Engine Inlets," 55th AIAA Aerospace Sciences Meeting, pp. 1-14.

[27] Bedick, C., Ferguson, D. H., Billups, D., O’Meara, B., Strakey, P., and Nix, A. C., 2018, "Characterization of Rotating Detonation Engine Injector Response Using Acetone PLIF in a Lab-Scale Experimental Testing Platform," 2018 Joint Propulsion Conference. 
[28] Sisler, A. T., 2016, "Experimental Investigation for Characterizing and Improving Inlet Designs in Rotating Detonation Engines," West Virginia University.

[29] Braun, E., Balcazar, T. S., Wilson, D. R., and Lu, F. K., 2012, "Experimental Study of a High-Frequency Fluidic Valve Fuel Injector,” J. Propuls. Power.

[30] Peace, J., Joshi, D., Lu, F. K., Peace, J. T., and Joshi, D. D., 2014, "Experimental Study of High-Frequency Fluidic Valve Injectors for Detonation Engine Applications.”

[31] Naples, A., Hoke, J., and Schauer, F., "Experimental Investigation of a Rotating Detonation Engine Fuel Injector Temporal Response.”

[32] Anand, V., George, A., Driscoll, R., and Gutmark, E., 2016, "Analysis of Air Inlet and Fuel Plenum Behavior in a Rotating Detonation Combustor," Exp. Therm. Fluid Sci., 70, pp. $408-416$.

[33] Schwer, D., Corrigan, A., Taylor, B., and Kailasanath, K., 2013, “On Reducing Feedback Pressure in Rotating Detonation Engines," 51st AIAA Aerospace Sciences Meeting Including the New Horizons Forum and Aerospace Exposition.

[34] Schwer, D. A., and Kailasanath, K., "Effect of Inlet on Fill Region and Performance of Rotating Detonation Engines.”

[35] Rankin, B. A., Codoni, J. R., Cho, K. Y., Hoke, J., and Schauer, F. R., 2017, “Mid-Infrared Imaging of an Optically Accessible Non-Premixed Hydrogen-Air Rotating Detonation Engine," 55th AIAA Aerospace Sciences Meeting, American Institute of Aeronautics and Astronautics.

[36] Gejji, R. M., Walters, I. V., Lemcherfi, A., Sardeshmukh, S. V., Heister, S. D., and 
Slabaugh, C. D., 2018, “Transducer Installation Effects on Pressure Measurements in PGC Devices," 2018 AIAA Aerospace Sciences Meeting.

[37] Naples, A., Hoke, J. L., and Schauer, F. R., 2018, "Quantification of Infinite Line Pressure Probe Response to Shocks and Detonation Waves," 2018 AIAA Aerospace Sciences Meeting, pp. 1-12.

[38] Shank, J. C., King, P. I., Karnesky, J., Schauer, F. R., and Hoke, J. L., 2012, “Development and Testing of a Modular Rotating Detonation Engine," 50th AIAA Aerospace Sciences Meeting.

[39] Bykovskii, F. A., Zhdan, S. A., Vedernikov, E. F., and Samsonov, A. N., 2017, "Scaling Factor in Continuous Spin Detonation of Syngas-air Mixtures,” Combust. Explos. Shock Waves, 53(2), pp. 187-198.

[40] Naples, A., Knisely, A. M., Hoke, J., and Schauer, F., 2019, "Infinite Line Pressure Probe and Flush Transducer Measurements in a Rotating Detonation Engine Channel," AIAA Scitech 2019 Forum.

[41] Bluemner, R., Bohon, M. D., Paschereit, C. O., and Gutmark, E. J., 2018, "Single and Counter-Rotating Wave Modes in an RDC,” 2018 AIAA Aerosp. Sci. Meet., (January).

[42] Fotia, M. L., Hoke, J. L., and Schauer, F., "Propellant Plenum Dynamics in a TwoDimensional Rotating Detonation Experiment." 


\section{Appendix}

\subsection{Appendix A: BSRDE Uncertainty}

\begin{tabular}{|c|c|c|c|c|c|c|c|c|c|c|c|c|c|c|}
\hline & \multirow[t]{2}{*}{ BIAS UNCERTAINTY } & & \multirow[b]{2}{*}{ Measured Value } & \multirow[b]{2}{*}{ Units } & \multirow[b]{2}{*}{ Uncertainty \% } & \multirow[b]{2}{*}{ Uncertainty } & \multicolumn{2}{|c|}{ Perturbed Variables } & \multicolumn{3}{|c|}{ Perturbed Equivalence Ratio Values } & \multicolumn{3}{|c|}{ Differences } \\
\hline & & & & & & & - & + & & + & & + &. & Total \\
\hline \multirow{3}{*}{$P_{\text {OH }}$} & Dynamic Channel Pressure & PCB CA 102-B06 & 57895.2 & Pa (gauge) & $1.000 \%$ & 579.0 & 57316.25 & 58474.15 & $1.92 E-08$ & $1.93 \mathrm{E}-08$ & $1.91 \mathrm{E}-08$ & $9.66 \mathrm{E}-11$ & $-9.66 \mathrm{E}-11$ & $9.66 \mathrm{E}-11$ \\
\hline & Back Pressure & Rosemount 1151GP8 & 14473.8 & Pa (gauge) & $0.750 \%$ & 108.6 & 14365.25 & 14582.35 & $1.92 E-08$ & $1.92 \mathrm{E}-08$ & $1.92 \mathrm{E}-08$ & $1.81 E-11$ & $-1.81 \mathrm{E}-11$ & $1.81 \mathrm{E}-11$ \\
\hline & Ambient Pressure & 42552.972 & & & & & & & & & & & & \\
\hline \multirow[b]{2}{*}{$p_{2}$} & Plenum Supply Pressure & Kulite 8442-4-140 & 173685.6 & $\mathrm{~Pa}$ (absolute) & $0.500 \%$ & 868.4 & 172817.17 & 174554.03 & $1.92 \mathrm{E}-08$ & $1.91 \mathrm{E}-08$ & $1.93 \mathrm{E}-08$ & $-9.54 E-11$ & $9.64 \mathrm{E}-11$ & $9.59 \mathrm{E}-1 \mathrm{i}$ \\
\hline & Plenum Temperature & Omega KQXL-116U-6 & 347.04 & $\mathrm{~K}$ & $0.750 \%$ & 2.6 & 344.44 & 349.64 & $1.92 E-08$ & $1.93 \mathrm{E}-08$ & $1.90 \mathrm{E}-08$ & $1.44 E-10$ & $-1.44 \mathrm{E}-10$ & $1.44 \mathrm{E}-1 \mathrm{C}$ \\
\hline \multirow{18}{*}{$Q_{0}$} & Differential Pressure across Orifice Flow Meter & & 217107 & Pa (gauge) & $0.075 \%$ & 162.8 & 216944.17 & 217269.83 & $1.92 E-08$ & $1.92 \mathrm{E}-08$ & $1.92 \mathrm{E}-08$ & $-1.12 E-11$ & $1.12 \mathrm{E}-11$ & $1.12 \mathrm{E}-1 \mathrm{~T}$ \\
\hline & Differential Pressure across Orifice Flow Meter & & 217107 & $\mathrm{~Pa}$ (gauge) & $0.075 \%$ & 162.8 & 216944.17 & 217269.83 & $1.92 E-08$ & $1.92 \mathrm{E}-08$ & $1.92 \mathrm{E}-08$ & $-1.74 E-12$ & $1.74 E-12$ & $1.74 E-12$ \\
\hline & \begin{tabular}{|l} 
Differential Pressure across Orifice Flow Meter \\
\end{tabular} & & 217107 & $\mathrm{~Pa}$ (gauge) & $0.075 \%$ & 162.8 & 216944.17 & 217269.83 & $1.92 E-08$ & $1.92 \mathrm{E}-08$ & $1.92 \mathrm{E}-08$ & $-1.43 E-12$ & $1.43 \mathrm{E}-12$ & $1.43 \mathrm{E}-12$ \\
\hline & \begin{tabular}{|l|} 
Reference Pressure for Flow Correction \\
\end{tabular} & Rosemount 1151GP8 & 31842.36 & $\mathrm{~Pa}$ (absolute) & $0.100 \%$ & 31.8 & 31810.52 & 31874.20 & $1.92 \mathrm{E}-08$ & $1.92 \mathrm{E}-08$ & $1.92 \mathrm{E}-08$ & $-1.49 \mathrm{E}-11$ & $1.50 \mathrm{E}-11$ & $1.50 \mathrm{E}-1 \mathrm{1}$ \\
\hline & Reference Pressure for Flow Correction & \begin{tabular}{|l|} 
Rosemount 1151GP8 \\
\end{tabular} & 31842.36 & $\mathrm{~Pa}$ (absolute) & $0.100 \%$ & 31.8 & 31810.52 & 31874.20 & $1.92 E-08$ & $1.92 \mathrm{E}-08$ & $1.92 \mathrm{E}-08$ & $-2.32 \mathrm{E}-12$ & $2.32 \mathrm{E}-12$ & $2.32 \mathrm{E}-12$ \\
\hline & \begin{tabular}{|l|} 
Reference Pressure for Flow Correction \\
\end{tabular} & Rosemount 1151GP8 & 31842.36 & $\mathrm{~Pa}$ (absolute) & $0.100 \%$ & 31.8 & 31810.52 & 31874.20 & $1.92 E-08$ & $1.92 \mathrm{E}-08$ & $1.92 \mathrm{E}-08$ & $-1.91 E-12$ & $1.91 \mathrm{E}-12$ & $1.91 \mathrm{E}-12$ \\
\hline & Reference Temperature for Flow Correction & K-Type Thermocoupl, & 343.15 & $\mathrm{~K}$ & $0.750 \%$ & 2.6 & 340.58 & 345.72 & $1.92 E-08$ & $1.93 \mathrm{E}-08$ & $1.91 \mathrm{E}-08$ & $1.12 E-10$ & $-1.12 \mathrm{E}-10$ & $1.12 \mathrm{E}-1 \mathrm{C}$ \\
\hline & Reference Temperature for Flow Correction & K-Type Thermocoupl, & 343.15 & $\mathrm{k}$ & $0.750 \%$ & 2.6 & 340.58 & 345.72 & $1.92 E-08$ & $1.92 \mathrm{E}-08$ & $1.92 \mathrm{E}-08$ & $1.73 E-11$ & $-1.75 E-11$ & $1.74 \mathrm{E}-1$ \\
\hline & Reference Temperature for Flow Correction & K-Type Thermocoupl, & 343.15 & $\mathrm{k}$ & $0.750 \%$ & 2.6 & 340.58 & 345.72 & $1.92 E-08$ & $1.92 \mathrm{E}-08$ & $1.92 \mathrm{E}-08$ & $1.42 E-11$ & $-1.44 E-11$ & $1.43 \mathrm{E}-1 \mathrm{I}$ \\
\hline & & & & & & & & & & & & & & $2.08 E-10$ \\
\hline & & & & & & & & & & & & & $\Delta \Pi_{2} / \Pi_{2}$ & $1.09 \%$ \\
\hline & PRECISION UNCERTAINTY & & & \multicolumn{2}{|c|}{ BIAS UNCERTAINTY } & & & & & & & & & \\
\hline & \begin{tabular}{|c|} 
Mean \\
\end{tabular} & $6.82 \mathrm{E}-07$ & & $\Delta \Pi_{2} / \Pi_{2}$ & $1.09 \%$ & & Constant & & & & & & & \\
\hline & Standard Deviation & $3.62 \mathrm{E}-08$ & & \multicolumn{2}{|c|}{ PRECISION UNCERTAINTY } & & co & 0.6 & & B1 & 0.885 & d1 & 0.0450 & $\mathrm{~m}$ \\
\hline & $\mathrm{N}$ & 696 & & $\Delta \Pi_{2} / \Pi_{2}$ & $5.31 \%$ & & d & 0.04826 & $\mathrm{~m}$ & B2 & 0.438 & A1 & $1.59 \mathrm{E}-03$ & $m^{\wedge} 2$ \\
\hline & & & & \multicolumn{2}{|c|}{ TOTAL UNCERTAINTY } & & D & 0.0508 & $\mathrm{~m}$ & B3 & 0.399 & $\mathrm{~d} 2$ & 0.0223 & $\mathrm{~m}$ \\
\hline & $\Delta \Pi_{2} / \Pi_{2}$ & $5.31 \%$ & & $\Delta \Pi_{2} / \Pi_{2}$ & $5.42 \%$ & & Ao & 0.002 & $m^{\wedge} 2$ & & & A2 & $3.90 E-04$ & $\mathrm{~m}^{\wedge} 2$ \\
\hline & & & & & & & $\mathrm{R}$ & 287 & $\mathrm{~m} 2 /(\mathrm{s} 2 \mathrm{~K})$ & & & d3 & 0.0202 & $\mathrm{~m}$ \\
\hline & & & & & & & B & 0.95 & & & & $\mathrm{~A}^{3}$ & $3.22 \mathrm{E}-04$ & $m^{\wedge} 2$ \\
\hline
\end{tabular}




\begin{tabular}{|c|c|c|c|c|c|c|c|c|c|c|c|c|c|c|c|c|c|c|c|c|c|c|}
\hline & & \multicolumn{3}{|c|}{ Change Channel Dynamic Pressure } & \multicolumn{3}{|c|}{ Change Back Pressure } & \multicolumn{3}{|c|}{ Change Air Plenum Supply Pressure } & \multicolumn{3}{|c|}{ Change Air Plenum Supply Temperature } & \multicolumn{3}{|c|}{ Change Air Pressue 1} & \multicolumn{3}{|c|}{ Change Air Pressue 2} & \multicolumn{3}{|c|}{ Change Air Pressue 3} \\
\hline & & & Uncertainty & 578.95200 & & Uncertainty: & 108.554 & & Uncertainty & 868.4 & & Uncertainty & 2.6 & & Uncertainty: & 162.8303 & & Uncertainty: & 162.83025 & & Uncertainty: & 162.83025 \\
\hline & & Pch & Pcht & Pch- & $\mathrm{Pb}$ & $\mathrm{Pb}+_{+}$ & $\mathrm{Pb}$ - & $\mathrm{Ppl}$ & Ppl+ & Ppl- & $\mathrm{Tpl}$ & Tpl+ & \begin{tabular}{|c|} 
Tpl- \\
\end{tabular} & $P_{11}$ & $\mathrm{P}_{11^{+}}$ & $P_{11}-$ & $P_{21}$ & $\mathrm{P}_{21^{+}}$ & \begin{tabular}{|l|}
$P_{21^{-}}$ \\
\end{tabular} & $P_{31}$ & $P_{33^{+}}$ & $P_{31-}$ \\
\hline & & 57895.20 & 58474.15 & 57316.25 & 14473.8 & 14582.4 & 14365.2 & 173685.6 & 174554.0 & 172817.2 & 347.04 & 349.6 & 344.4 & 217107 & 217269.83 & 216944.17 & 217107 & 217269.83 & 216944.17 & 217107 & 217269.83 & 216944.1698 \\
\hline \multirow[t]{5}{*}{ Air Flow 1} & $P_{1}$ & 217107 & 217107 & 217107 & 217107 & 217107 & 217107 & 217107 & 217107 & 217107 & 217107 & 217107 & 217107 & 217107 & 217269.83 & 216944.17 & 217107 & 217107 & 217107 & 217107 & 217107 & 217107 \\
\hline & $D P_{1}$ & 31842.36 & 31842.36 & 31842.36 & 31842.36 & 31842.36 & 31842.36 & 31842.36 & 31842.36 & 31842.36 & 31842.36 & 31842.36 & 31842.36 & 31842.36 & 31842.36 & \begin{tabular}{|l|}
31842.36 \\
\end{tabular} & 31842.36 & 31842.36 & 31842.36 & 31842.36 & 31842.36 & 31842.36 \\
\hline & $T_{T}$ & 343.15 & 343.15 & 343.15 & 343.15 & 343.15 & 343.15 & 343.15 & 343.15 & 343.15 & 343.15 & 343.15 & 343.15 & 343.15 & 343.15 & 343.15 & 343.15 & 343.15 & 343.15 & 343.15 & 343.15 & 343.15 \\
\hline & $\rho$ & 2.20449 & 2.20449 & 2.20449 & 2.20449 & 2.20449 & 2.20449 & 2.20449 & 2.20449 & 2.20449 & 2.20449 & 2.20449 & 2.20449 & 2.20449 & 2.20614 & 2.20284 & 2.20449 & 2.20449 & 2.20449 & 2.20449 & 2.20449 & 2.20449 \\
\hline & $Q$ & 0.45 & 0.45 & 0.45 & 0.45 & 0.45 & 0.45 & 0.45 & 0.45 & 0.45 & 0.45 & 0.45 & 0.45 & 0.45 & 0.45 & 0.45 & 0.45 & 0.45 & 0.45 & 0.45 & 0.45 & 0.45 \\
\hline & & & & & & & & & & & & & & & & & & & & & & \\
\hline \multirow[t]{5}{*}{ Air Flow 2} & $P_{1}$ & 217107 & 217107 & 217107 & 217107 & 217107 & 217107 & 217107 & 217107 & 217107 & 217107 & 217107 & 217107 & 217107 & 217107 & 217107 & 217107 & 217269.83 & 216944.17 & 217107 & 217107 & 217107 \\
\hline & $\mathrm{DP}_{2}$ & 31842.36 & 31842.36 & 31842.36 & 31842.36 & 31842.36 & 31842.36 & 31842.36 & 31842.36 & 31842.36 & 31842.36 & 31842.36 & 31842.36 & 31842.36 & 31842.36 & 31842.36 & 31842.36 & 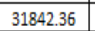 & \begin{tabular}{|l|l}
31842.36 \\
\end{tabular} & 31842.36 & 31842.36 & 31842.36 \\
\hline & $T_{T}$ & 343.15 & \begin{tabular}{|l|}
343.15 \\
\end{tabular} & 343.15 & 343.15 & 343.15 & 343.15 & 343.15 & 343.15 & 343.15 & 343.15 & 343.15 & 343.15 & 343.15 & 343.15 & 343.15 & 343.15 & 343.15 & \begin{tabular}{|l|}
343.15 \\
\end{tabular} & 343.15 & 343.15 & 343.15 \\
\hline & $p$ & 2.20449 & 2.20449 & 2.20449 & 2.20449 & 2.20449 & 2.20449 & 2.20449 & 2.20449 & 2.20449 & 2.20449 & 2.20449 & 2.20449 & 2.20449 & 2.20449 & 2.20449 & 2.20449 & 2.20614 & 2.20284 & 2.20449 & 2.20449 & 2.20449 \\
\hline & $Q$ & 0.07 & 0.07 & 0.07 & 0.07 & 0.07 & 0.07 & 0.07 & 0.07 & 0.07 & 0.07 & 0.07 & 0.07 & 0.07 & 0.07 & 0.07 & 0.07 & 0.07 & 0.07 & 0.07 & 0.07 & 0.07 \\
\hline \multirow{6}{*}{ Air Flow 3} & & & & & & & & & & & & & & & & & & & & & & \\
\hline & $p_{1}$ & 217107 & 217107 & 217107 & 217107 & 217107 & 217107 & 217107 & 217107 & 217107 & 217107 & 217107 & 217107 & 217107 & 217107 & 217107 & 217107 & 217107 & 217107 & 217107 & 217270 & 216944 \\
\hline & $D P_{3}$ & 31842.36 & \begin{tabular}{|l|l|}
1842.36 \\
\end{tabular} & 31842.36 & 31842.36 & 31842.36 & 31842.36 & 31842.36 & 31842.36 & 31842.36 & 31842.36 & 31842.36 & 31842.36 & 31842.36 & 31842.36 & 31842.36 & 31842.36 & 31842.36 & 31842.36 & 31842.36 & 31842.36 & 31842.36 \\
\hline & $T_{T}$ & 343.15 & 343.15 & 343.15 & 343.15 & 343.15 & 343.15 & 343.15 & 343.15 & 343.15 & 343.15 & 343.15 & 343.15 & 343.15 & 343.15 & 343.15 & 343.15 & 343.15 & 343.15 & 343.15 & 343.15 & 343.15 \\
\hline & $\rho$ & 2.20449 & 2.20449 & 2.20449 & 2.20449 & 2.20449 & 2.20449 & 2.20449 & 2.20449 & 2.20449 & 2.20449 & 2.20449 & 2.20449 & 2.20449 & 2.20449 & 2.20449 & 2.20449 & 2.20449 & 2.20449 & 2.20449 & 2.20614 & 2.20284 \\
\hline & $Q$ & 0.06 & 0.06 & 0.06 & 0.06 & 0.06 & 0.06 & 0.06 & 0.06 & 0.06 & 0.06 & 0.06 & 0.06 & 0.06 & 0.06 & 0.06 & 0.06 & 0.06 & 0.06 & 0.06 & 0.06 & 0.06 \\
\hline & & & & & & & & & & & & & & & & & & & & & & \\
\hline \multirow[t]{3}{*}{ Air Plenum Density } & Ppl & 173685.6 & \begin{tabular}{|l|}
173685.6 \\
\end{tabular} & 173685.6 & 173685.6 & \begin{tabular}{|l|}
173685.6 \\
\end{tabular} & 173685.6 & \begin{tabular}{|l|}
173685.6 \\
\end{tabular} & \begin{tabular}{|l|l|}
174554.03 \\
\end{tabular} & \begin{tabular}{|l|}
172817.172 \\
\end{tabular} & 173685.6 & 173685.6 & 173685.6 & 173685.6 & 173685.6 & 173685.6 & 173685.6 & \begin{tabular}{|l|}
173685.6 \\
\end{tabular} & \begin{tabular}{|l|}
173685.6 \\
\end{tabular} & 173685.6 & 173685.6 & 173685.6 \\
\hline & Tpl & 347.03889 & \begin{tabular}{|l|}
347.03889 \\
\end{tabular} & 347.0388889 & 347.03889 & 347.03889 & 347.0388889 & \begin{tabular}{|l|}
347.03889 \\
\end{tabular} & \begin{tabular}{|l|}
347.03889 \\
\end{tabular} & \begin{tabular}{|l|l|}
347.038889 \\
\end{tabular} & \begin{tabular}{|l|}
347.03889 \\
\end{tabular} & 349.6416806 & 344.4361 & 347.03889 & \begin{tabular}{|l|l}
347.0388899 \\
\end{tabular} & \begin{tabular}{|l|}
347.03889 \\
\end{tabular} & 347.0388889 & 347.03889 & \begin{tabular}{|l|}
347.03889 \\
\end{tabular} & 347.038889 & \begin{tabular}{|l|}
347.03889 \\
\end{tabular} & 347.0388889 \\
\hline & gpl & 1.743829 & 1.743829 & 1.743829 & 1.743829 & 1.743829 & \begin{tabular}{|l|l|}
.743829 \\
\end{tabular} & \begin{tabular}{|l|}
1.743829 \\
\end{tabular} & \begin{tabular}{|l|}
1.752548 \\
\end{tabular} & \begin{tabular}{|l|}
1.735109 \\
\end{tabular} & 1.743829 & 1.730847 & 1.757006 & 1.743829 & \begin{tabular}{|l|}
1.743829 \\
\end{tabular} & \begin{tabular}{|l|}
1.743829 \\
\end{tabular} & \begin{tabular}{|l|l|}
1.743829 \\
\end{tabular} & $\begin{array}{l}1.743829 \\
\end{array}$ & \begin{tabular}{|l|}
1.743829 \\
\end{tabular} & 1.743829 & \begin{tabular}{|l|}
1.743829 \\
\end{tabular} & 1.743829 \\
\hline & & & & & & & & & & & & & & & & & & & & & & \\
\hline \multirow[t]{8}{*}{ Channel Pressure } & Pch,d & 57895.20 & $\begin{array}{l}58474.15 \\
\end{array}$ & 57316.25 & 57895.20 & \begin{tabular}{|l|}
57895.20 \\
\end{tabular} & 57895.20 & 57895.20 & 57895.20 & 57895.20 & 57895.20 & 57895.20 & 57895.20 & 57895.20 & 57895.20 & 57895.20 & 57895.20 & 57895.20 & 57895.20 & 57895.20 & 57895.20 & 57895.20 \\
\hline & Pback & $\begin{array}{l}14473.8 \\
\end{array}$ & \begin{tabular}{|r|}
14473.8 \\
\end{tabular} & 14473.8 & $\begin{array}{r}14473.8 \\
\end{array}$ & 14582.354 & 14365.2465 & \begin{tabular}{|l|}
14473.8 \\
\end{tabular} & \begin{tabular}{|l|}
14473.8 \\
\end{tabular} & 14473.8 & 14473.8 & $\begin{array}{l}14473.8 \\
\end{array}$ & 14473.8 & \begin{tabular}{|l|}
14473.8 \\
\end{tabular} & 14473.8 & \begin{tabular}{|r|}
14473.8 \\
\end{tabular} & 14473.8 & $\begin{array}{r}14473.8 \\
\end{array}$ & 14473.8 & 14473.8 & 14473.8 & 14473.8 \\
\hline & Pamb & 42552.972 & \begin{tabular}{|l|l|}
42552.972 \\
\end{tabular} & 42552.972 & 42552.972 & 42552.972 & 42552.972 & \begin{tabular}{|l|}
42552.972 \\
\end{tabular} & \begin{tabular}{|l|l|}
42552.972 \\
\end{tabular} & 42552.972 & 42552.972 & 42552.972 & 42552.972 & \begin{tabular}{|l|}
42552.972 \\
\end{tabular} & 42552.972 & \begin{tabular}{|l|l|}
42552.972 \\
\end{tabular} & 42552.972 & 42552.972 & 42552.972 & 42552.972 & 42552.972 & 42552.972 \\
\hline & & & & & & & & & & & & & & & & & & & & & & \\
\hline & $Q_{\text {Si TOTN }}$ & 0.579 & 0.579 & 0.579 & 0.579 & & $\begin{array}{ll}0.579 \\
\end{array}$ & 0.579 & 0.579 & 0.579 & 0.579 & 0.579 & 0.579 & 0.579 & 0.579 & 0.579 & 0.579 & 0.579 & 0.579 & 0.579 & 0.579 & 0.579 \\
\hline & Pch & 114921.97 & 115500.92 & 114343.02 & 114921.97 & 115030.53 & 114813.42 & 114921.97 & \begin{tabular}{|l|l|}
114921.97 \\
\end{tabular} & \begin{tabular}{|l|l|}
114921.97 \\
\end{tabular} & \begin{tabular}{|l|l|}
114921.97 \\
\end{tabular} & 114921.97 & 114921.97 & 114921.97 & \begin{tabular}{|l|}
114921.97 \\
\end{tabular} & 114921.97 & 114921.97 & 114921.97 & 114921.97 & 114921.97 & 114921.97 & \\
\hline & pa & 1.74 & 1.74 & 1.74 & 1.74 & 1.74 & 1.74 & 1.74 & 1.75 & \begin{tabular}{|l|}
1.74 \\
\end{tabular} & 1.74 & \begin{tabular}{l|l}
1.73 \\
\end{tabular} & 1.76 & 1.74 & 1.74 & 1.74 & 1.74 & 1.74 & 1.74 & 1.74 & 1.74 & 1.74 \\
\hline & $\mathrm{Pi} 2$ & $1.92 \mathrm{E}-08$ & $1.93 \mathrm{E}-08$ & $1.91 \mathrm{E}-08$ & $1.92 E-08$ & $1.922-08$ & $1.922-08$ & $1.92 E-08$ & $1.91 \mathrm{E}-08$ & $1.93 \mathrm{E}-08$ & 1.92E-08 & $1.93 E-08$ & $1.90 \mathrm{E}-08$ & $1.92 E-08$ & $1.92 E-08$ & $1.92 \mathrm{E}-08$ & $1.92 \mathrm{E}-08$ & $1.92 E-08$ & 1.92E-08 & $1.92 E-08$ & $1.92 \mathrm{E}-08$ & $1.92 E-08$ \\
\hline
\end{tabular}




\begin{tabular}{|c|c|c|c|c|c|c|c|c|c|c|c|c|c|c|c|c|c|c|c|}
\hline & & \multicolumn{3}{|c|}{ Change Differential Pressure 1} & \multicolumn{3}{|c|}{ Change Differential Pressure 2} & \multicolumn{3}{|c|}{ Change Differential Pressure 3} & \multicolumn{3}{|c|}{ Change Total Temperature 1} & \multicolumn{3}{|c|}{ Change Total Temperature 2} & \multicolumn{3}{|c|}{ Change Total Temperature 3} \\
\hline & & & \begin{tabular}{|l|} 
Uncertainty \\
\end{tabular} & 31.84236 & & Uncertainty: & \begin{tabular}{|r|}
31.8424 \\
\end{tabular} & & Uncertainty: & 31.84236 & & \begin{tabular}{|l|} 
Uncertainty \\
\end{tabular} & 2.6 & & \begin{tabular}{|l|l|} 
Uncertainty \\
\end{tabular} & 2.6 & & Uncertainty & 2.6 \\
\hline & & $\mathrm{DP}_{1}$ & $\mathrm{DP}_{1}+$ & $\mathrm{DP}_{1^{-}}$ & $\mathrm{DP}_{2}$ & $\mathrm{DP}_{2^{+}}$ & $\mathrm{DP}_{2}$ & $\mathrm{DP}_{3}$ & $\mathrm{DP}_{3^{+}}$ & $\mathrm{DP}_{5^{-}}$ & $\mathrm{T}_{\mathrm{T} 1}$ & $\mathrm{~T}_{\mathrm{T1}^{+}}$ & $\mathrm{T}_{\mathrm{Tt}^{-}}$ & $\mathrm{T}_{\mathrm{T} 2}$ & $\mathrm{~T}_{\mathrm{T}^{+}}+$ & $\mathrm{T}_{\mathrm{T} 2^{-}}$ & $\mathrm{T}_{\mathrm{T} 2}$ & $\mathrm{~T}_{\mathrm{T}^{+}}{ }^{+}$ & $\mathrm{T}_{T_{2} \cdot}$ \\
\hline & & 31842.36 & 31874.202 & 31810.51764 & 31842.36 & \begin{tabular}{|l|l|}
31874.202 \\
\end{tabular} & 31810.51764 | & 31842.36 & 31874.202 & 31810.5176 & 343.15 & 345.723625 & 340.57638 & 343.15 & 345.723625 & 340.57638 & 343.15 & 345.72363 & 340.57638 ( \\
\hline \multirow[t]{5}{*}{ Air Flow 1} & $P_{1}$ & 217107 & \begin{tabular}{|l|}
217107 \\
\end{tabular} & 217107 & 217107 & \begin{tabular}{l|l}
217107 \\
\end{tabular} & \begin{tabular}{|l|}
217107 \\
\end{tabular} & 217107 & 217107 & 217107 & 217107 & 217107 & 217107 & 217107 & 217107 & 217107 & 217107 & 217107 & 217107 \\
\hline & $D P_{1}$ & 31842.36 & 31874.202 & 31810.51764 & 31842.36 & 31842.36 & 31842.36 & 31842.36 & 31842.36 & 31842.36 & 31842.36 & 31842.36 & 31842.36 & 31842.36 & 31842.36 & 31842.36 & 31842.36 & 31842.36 & 31842.36 \\
\hline & $T_{T}$ & 343.15 & 343.15 & 343.15 & 343.15 & 343.15 & 343.15 & 343.15 & 343.15 & 343.15 & 343.15 & 345.723625 & 340.57638 & 343.15 & 343.15 & 343.15 & 343.15 & 343.15 & 343.15 \\
\hline & $p$ & 2.20449 & 2.20449 & 2.20449 & 2.20449 & 2.20449 & 2.20449 & 2.20449 & 2.20449 & 2.20449 & 2.20449 & \begin{tabular}{|l|}
2.18808 \\
\end{tabular} & \begin{tabular}{|l|}
2.22115 \\
\end{tabular} & 2.20449 & 2.20449 & 2.20449 & 2.20449 & 2.20449 & 2.20449 \\
\hline & $Q$ & 0.45 & 0.45 & 0.45 & 0.45 & 0.45 & 0.45 & 0.45 & 0.45 & 0.45 & 0.45 & 0.45 & 0.45 & 0.45 & 0.45 & 0.45 & 0.45 & 0.45 & 0.45 \\
\hline \multirow[t]{5}{*}{ Air Flow 2} & $P_{1}$ & 217107 & 217107 & 217107 & 217107 & 217107 & 217107 & 217107 & 217107 & 217107 & 217107 & 217107 & 217107 & 217107 & 217107 & 217107 & 217107 & 217107 & 217107 \\
\hline & $\mathrm{DP}_{2}$ & 31842.36 & 31842.36 & 31842.36 & 31842.36 & 31874.202 & \begin{tabular}{|l|}
31810.51764 \\
\end{tabular} & 31842.36 & 31842.36 & 31842.36 & 31842.36 & 31842.36 & 31842.36 & 31842.36 & 31842.36 & 31842.36 & 31842.36 & 31842.36 & 31842.36 \\
\hline & $T_{T}$ & 343.15 & 343.15 & 343.15 & 343.15 & \begin{tabular}{|l|l}
343.15 & \\
\end{tabular} & \begin{tabular}{|l|}
343.15 \\
\end{tabular} & 343.15 & 343.15 & 343.15 & 343.15 & 343.15 & 343.15 & 343.15 & 345.723625 & 340.57638 & 343.15 & 343.15 & 343.15 \\
\hline & $p$ & 2.20449 & 2.20449 & 2.20449 & 2.20449 & 2.20449 & 2.20449 & 2.20449 & 2.20449 & 2.20449 & 2.20449 & 2.20449 & 2.20449 & 2.20449 & \begin{tabular}{|l|}
2.18808 \\
\end{tabular} & 2.22115 & 2.20449 & 2.20449 & 2.20449 \\
\hline & $Q$ & 0.07 & 0.07 & 0.07 & 0.07 & 0.07 & 0.07 & 0.07 & 0.07 & 0.07 & 0.07 & 0.07 & 0.07 & 0.07 & 0.07 & 0.07 & 0.07 & 0.07 & 0.07 \\
\hline \multirow[t]{5}{*}{ Air Flow 3} & $P_{1}$ & 217107 & 217107 & 217107 & 217107 & 217107 & 217107 & 217107 & 217107 & 217107 & 217107 & 217107 & 217107 & 217107 & 217107 & 217107 & 217107 & 217107 & 217107 \\
\hline & $\mathrm{DP}_{3}$ & 31842.36 & 31842.36 & 31842.36 & 31842.36 & 31842.36 & 31842.36 & 31842.36 & 31874.202 & 31810.5176 & 31842.36 & 31842.36 & 31842.36 & 31842.36 & 31842.36 & 31842.36 & 31842.36 & 31842.36 & 31842.36 \\
\hline & $T_{T}$ & 343.15 & 343.15 & 343.15 & 343.15 & 343.15 & 343.15 & 343.15 & \begin{tabular}{|l|}
343.15 \\
\end{tabular} & \begin{tabular}{|l|}
343.15 \\
\end{tabular} & \begin{tabular}{|l|}
343.15 \\
\end{tabular} & 343.15 & 343.15 & 343.15 & 343.15 & 343.15 & 343.15 & 345.72363 & \begin{tabular}{|l|}
340.57638 \\
\end{tabular} \\
\hline & $p$ & 2.20449 & 2.20449 & 2.20449 & 2.20449 & 2.20449 & 2.20449 & 2.20449 & 2.20449 & 2.20449 & 2.20449 & 2.20449 & 2.20449 & 2.20449 & 2.20449 & 2.20449 & 2.20449 & 2.18808 & \begin{tabular}{|l|}
2.22115 \\
\end{tabular} \\
\hline & $Q$ & 0.06 & 0.06 & 0.06 & 0.06 & 0.06 & 0.06 & 0.06 & 0.06 & 0.06 & 0.06 & 0.06 & 0.06 & 0.06 & 0.06 & 0.06 & 0.06 & 0.06 & 0.06 \\
\hline \multirow{3}{*}{ Air Plenum Density } & $\mathrm{Ppl}$ & 173685.6 & 173685.6 & 173685.6 & 173685.6 & 173685.6 & 173685.6 & 173685.6 & 173685.6 & 173685.6 & 173685.6 & 173685.6 & 173685.6 & 173685.6 & 173685.6 & 173685.6 & 173685.6 & 173685.6 & 173685.6 \\
\hline & Tpl & 347.03889 & \begin{tabular}{|l|}
347.03889 \\
\end{tabular} & \begin{tabular}{|l|}
347.0388889 \\
\end{tabular} & 347.03889 & \begin{tabular}{|l|}
347.03889 \\
\end{tabular} & \begin{tabular}{|l|}
347.0388889 \\
\end{tabular} & \begin{tabular}{|l|}
347.03889 \\
\end{tabular} & 347.03889 & 347.038889 & \begin{tabular}{|l|}
347.03889 \\
\end{tabular} & \begin{tabular}{|l|}
347.0388889 \\
\end{tabular} & \begin{tabular}{|l|}
347.03889 \\
\end{tabular} & 347.03889 & \begin{tabular}{|l|}
447.038889 \\
\end{tabular} & 347.03889 & 347.0388889 & \begin{tabular}{|l|}
347.03889 \\
\end{tabular} & \begin{tabular}{|l|}
347.03889 \\
\end{tabular} \\
\hline & $\rho \mathrm{pl}$ & 1.743829 & \begin{tabular}{|l|l|}
1.743829 \\
\end{tabular} & 1.743829 & $\begin{array}{l}1.743829 \\
\end{array}$ & \begin{tabular}{|l|l}
1.743829 \\
\end{tabular} & \begin{tabular}{|l|}
1.743829 \\
\end{tabular} & \begin{tabular}{|l|}
1.743829 \\
\end{tabular} & 1.743829 & \begin{tabular}{|l|}
1.743829 \\
\end{tabular} & \begin{tabular}{|l|}
1.743829 \\
\end{tabular} & \begin{tabular}{|l|}
1.743829 \\
\end{tabular} & \begin{tabular}{|l|}
1.743829 \\
\end{tabular} & $\begin{array}{l}1.743829 \\
\end{array}$ & \begin{tabular}{|l|}
1.743829 \\
\end{tabular} & 1.743829 & \begin{tabular}{|l|}
1.743829 \\
\end{tabular} & \begin{tabular}{|l|l|}
1.743829 \\
\end{tabular} & \begin{tabular}{|l|}
1.743829 \\
\end{tabular} \\
\hline \multirow{8}{*}{ Channel Pressure } & Pchd & 57895.20 & 57895.20 & 57895,20 & 5789532 & 578953 & 5789520 & 578952 & 578953 & 5789520 & 578953 & 5789523 & 5789520 & 578952 & & 5789522 & 5789522 & & 578952 \\
\hline & Pback & 14473.8 & 14473.8 & 14473.8 & 14473.8 & \begin{tabular}{l|}
14473.8 \\
\end{tabular} & 14473.8 & 14473.8 & 14473.8 & 14473.8 & 14473.8 & \begin{tabular}{|l|}
14473.8 \\
\end{tabular} & $\begin{array}{r}14473.8 \\
1473.8 \\
\end{array}$ & $\begin{array}{r}5 / 8953.20 \\
14473.8 \\
\end{array}$ & \begin{tabular}{r|}
5895.20 \\
14473.8 \\
\end{tabular} & $\begin{array}{r}58957.20 \\
14473.8 \\
\end{array}$ & $\begin{array}{r}5 / 895.20 \\
14473.8 \\
\end{array}$ & \begin{tabular}{r|}
5895.20 \\
14473.8 \\
\end{tabular} & $\begin{array}{r}5 / 895.20 \\
14473.8 \\
\end{array}$ \\
\hline & Pamb & 42552.972 & \begin{tabular}{|l|l|}
42552.972 \\
\end{tabular} & 42552.972 & 42552.972 & \begin{tabular}{|l|}
42552.972 \\
\end{tabular} & 42552.972 & 42552.972 & 42552.972 & 42552.972 & 42552.972 & 42552.972 & \begin{tabular}{|l|l|} 
& 42552.972 \\
\end{tabular} & 42552.972 & 42552.972 & 42552.972 & 42552.972 & 42552.972 & 42552.972 \\
\hline & & & & & & & & & & & & & & & & & & & \\
\hline & $Q_{\text {ir TOTAL }}$ & 0.579 & 0.579 & 0.579 & 0.579 & 0.579 & 0.579 & 0.579 & 0.579 & 0.579 & 0.579 & 0.577 & 0.580 & 0.579 & 0.578 & 0.579 & 0.579 & 0.579 & 0.579 \\
\hline & Pch & 114921.97 & \begin{tabular}{|l|l|}
114921.97 \\
\end{tabular} & 114921.97 & \begin{tabular}{|l|l|}
114921.97 \\
\end{tabular} & 114921.97 & 114921.97 & \begin{tabular}{|l|l|l|l|l|}
114921 \\
\end{tabular} & \begin{tabular}{|l|}
114921.97 \\
\end{tabular} & \begin{tabular}{|l|l|}
114921.97 \\
\end{tabular} & \begin{tabular}{|l|l|l|l|l|}
114921 \\
\end{tabular} & 114921.97 & \begin{tabular}{|l|l|}
114921.97 \\
\end{tabular} & \begin{tabular}{|l|l|}
114921.97 \\
\end{tabular} & 1114921.97 & $\begin{array}{l}114921.97 \\
\end{array}$ & \begin{tabular}{|l|l|}
114921.97 \\
\end{tabular} & \begin{tabular}{|l|l|}
114921.97 \\
\end{tabular} & \begin{tabular}{|l|}
114921.97 \\
\end{tabular} \\
\hline & $\rho a$ & 1.74 & \begin{tabular}{|l|}
1.74 \\
\end{tabular} & \begin{tabular}{l|l}
1.74 \\
\end{tabular} & 1.74 & \begin{tabular}{l|l}
1.74 \\
\end{tabular} & $\begin{array}{ll}1.74 \\
\end{array}$ & \begin{tabular}{|l|}
1.74 \\
\end{tabular} & \begin{tabular}{|l|l|}
1.74 \\
\end{tabular} & \begin{tabular}{|l|}
1.74 \\
\end{tabular} & \begin{tabular}{|l|}
1.74 \\
\end{tabular} & \begin{tabular}{l|l}
1.74 \\
\end{tabular} & \begin{tabular}{|l|}
1.74 \\
\end{tabular} & 1.74 & 1.74 & 1.74 & \begin{tabular}{l|l|}
1.74 \\
\end{tabular} & \begin{tabular}{|l|}
1.74 \\
\end{tabular} & 1.74 \\
\hline & $\mathrm{Pi} 2$ & $1.92 E-08$ & $1.92 E-08$ & $1.92 E-08$ & $1.92 \mathrm{E}-08$ & $1.92 E-08$ & $1.92 \mathrm{E}-08$ & $1.92 \mathrm{E}-08$ & $1.92 \mathrm{E}-08$ & $1.92 \mathrm{E}-08$ & $1.92 \mathrm{E}-08$ & $1.93 \mathrm{E}-08$ & $1.91 \mathrm{E}-08$ & $1.92 E-08$ & $1.92 \mathrm{E}-08$ & $1.92 \mathrm{E}-08$ & $1.92 \mathrm{E}-08$ & $1.92 \mathrm{E}-08$ & $1.92 \mathrm{E}-08$ \\
\hline
\end{tabular}




\subsection{Appendix B: LSRDE Uncertainty}

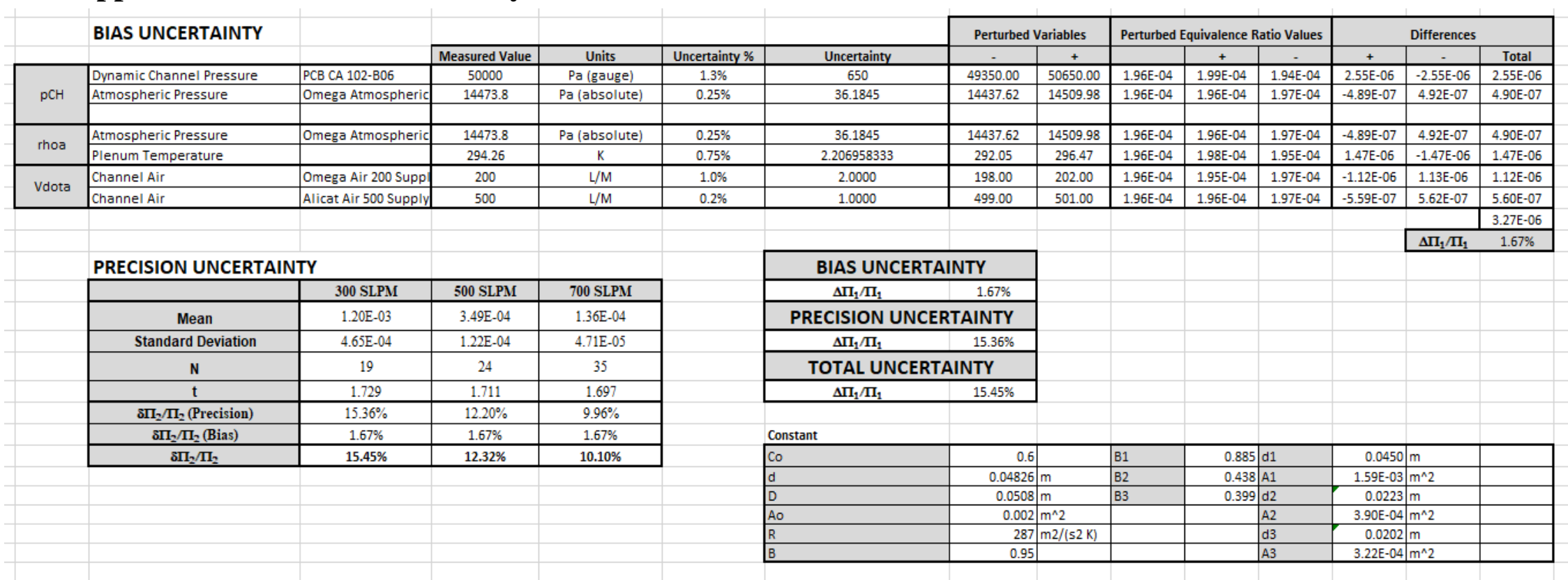

\begin{tabular}{|c|c|c|c|c|c|c|c|c|c|c|c|c|c|c|c|c|c|c|}
\hline & \multicolumn{3}{|c|}{ Change Channel Dynamic Pressure } & \multicolumn{3}{|c|}{ Change Atmospheric Pressure } & \multicolumn{3}{|c|}{ Change Atmospheric Pressure (2) } & \multicolumn{3}{|c|}{ Change Atmospheric Temperature } & \multicolumn{3}{|c|}{ Change 200 Air } & \multirow{2}{*}{\multicolumn{3}{|c|}{$\begin{array}{l}\text { Change } 500 \text { Air } \\
\text { Uncertainty: }\end{array}$}} \\
\hline & & Uncertainty & 650.00000 & & Uncertainty: & $\quad 36.185$ & & Uncertainty & 36.2 & & Uncertainty & 2.2 & & Uncertainty: & 2.0000 & & & \\
\hline & Pch & Pcht+ & $\begin{array}{ll}\text { Pch- } \\
\end{array}$ & Ps & $\mathrm{Ps}_{\mathrm{st}}$ & $\begin{array}{ll}\text { Ps- } \\
\end{array}$ & Ps & \begin{tabular}{|c|}
$P_{5+}$ \\
\end{tabular} & \begin{tabular}{|l|} 
Ps- \\
\end{tabular} & $\mathrm{T}$ & $\mathrm{T}_{+}$ & $\mathrm{T}-$ & $Q_{200}$ & $\mathrm{Q}_{200^{+}}$ & $\mathrm{Q}_{200}-$ & $Q_{500}$ & $Q_{500^{+}}$ & $Q_{500^{-}}$ \\
\hline & 50000.00 & 50650.00 & 49350.00 & 14473.8 & 14510.0 & 14437.6 & 14473.8 & 14510.0 & 14437.6 & 294.26 & 296.5 & 292.1 & 200 & 202 & 198 & 500 & 501 & 499 \\
\hline$P_{c h}$ & 50000 & 50650.00 & 49350.00 & 50000 & 50000 & 50000 & 50000 & 50000 & 50000 & 50000 & 50000 & 50000 & 50000 & 50000 & 50000 & 50000 & 50000 & 50000 \\
\hline$P_{0}$ & 14473.8 & 14473.8 & 14473.8 & 14473.8 & 14509.985 & 14437.6155 & 14473.8 & \begin{tabular}{|l|}
14509.985 \\
\end{tabular} & 14437.6155 & 14473.8 & 14473.8 & 14473.8 & 14473.8 & 14473.8 & 14473.8 & 14473.8 & 14473.8 & 14473.8 \\
\hline $\mathrm{T}_{0}$ & 294.26 & 294.26111 & 294.2611111 & 294.26 & 294.26111 & 294.2611111 & 294.26 & \begin{tabular}{|l|}
294.26111 \\
\end{tabular} & 294.261111 & 294.26 & 296.47 & 292.05 & 294.26 & 294.26 & 294.26111 & 294.26 & 294.26 & 294.26111 \\
\hline$a_{200}$ & 200.00000 & 200.00000 & 200.00000 & 200.00000 & 200.00000 & 200.00000 & 200.00000 & \begin{tabular}{|l|}
200.00000 \\
\end{tabular} & \begin{tabular}{|l|}
200.00000 \\
\end{tabular} & 200.00000 & 200.00000 & 200.00000 & 200.00000 & 202.00000 & 198.00000 & 200.00000 & 200.00000 & 200.00000 \\
\hline$Q_{500}$ & 500.00 & 500.00 & 500.00 & 500.00 & \begin{tabular}{|l|}
500.00 \\
\end{tabular} & 500.00 & 500.00 & 500.00 & 500.00 & 500.00 & 500.00 & 500.00 & 500.00 & $\begin{array}{r}500.00 \\
\end{array}$ & 500.00 & 500.00 & 501.00 & 499.00 \\
\hline$Q_{\text {air TOTAL }}$ & 0.0117 & 0.0117 & 0.0117 & 0.0117 & 0.0117 & 0.0117 & 0.0117 & 0.0117 & 0.0117 & 0.0117 & 0.0117 & 0.0117 & 0.0117 & 0.0117 & 0.0116 & 0.0117 & 0.0117 & 0.0117 \\
\hline Pch & 50000 & 50650 & 49350 & 50000 & 50000 & 50000 & 50000 & 50000 & 50000 & 50000 & 50000 & 50000 & 50000 & 50000 & 50000 & 50000 & 50000 & 50000 \\
\hline$\rho a$ & 0.17138 & 0.171383 & 0.171383023 & 0.17138 & 0.1718115 & 0.170954566 & 0.17138 & \begin{tabular}{|l|}
0.1718115 \\
\end{tabular} & \begin{tabular}{|l|l|l|l|l|l|l} 
\\
\end{tabular} & 0.17138 & \begin{tabular}{|l|l|}
0.170107219 \\
\end{tabular} & 0.1726781 & 0.17138 & 0.17138302 & 0.171383 & 0.17138 & 0.171383 & 0.171383 \\
\hline$\Pi_{2}$ & 0.00020 & 0.00020 & 0.00019 & 0.00020 & 0.00020 & 0.00020 & 0.00020 & 0.00020 & 0.00020 & 0.00020 & 0.00020 & 0.00019 & 0.00020 & 0.00020 & 0.00020 & 0.00020 & 0.00020 & 0.00020 \\
\hline
\end{tabular}




\title{
10.3 Appendix C: BSRDE MATLAB Code
}

\author{
clear, close all, clc
}

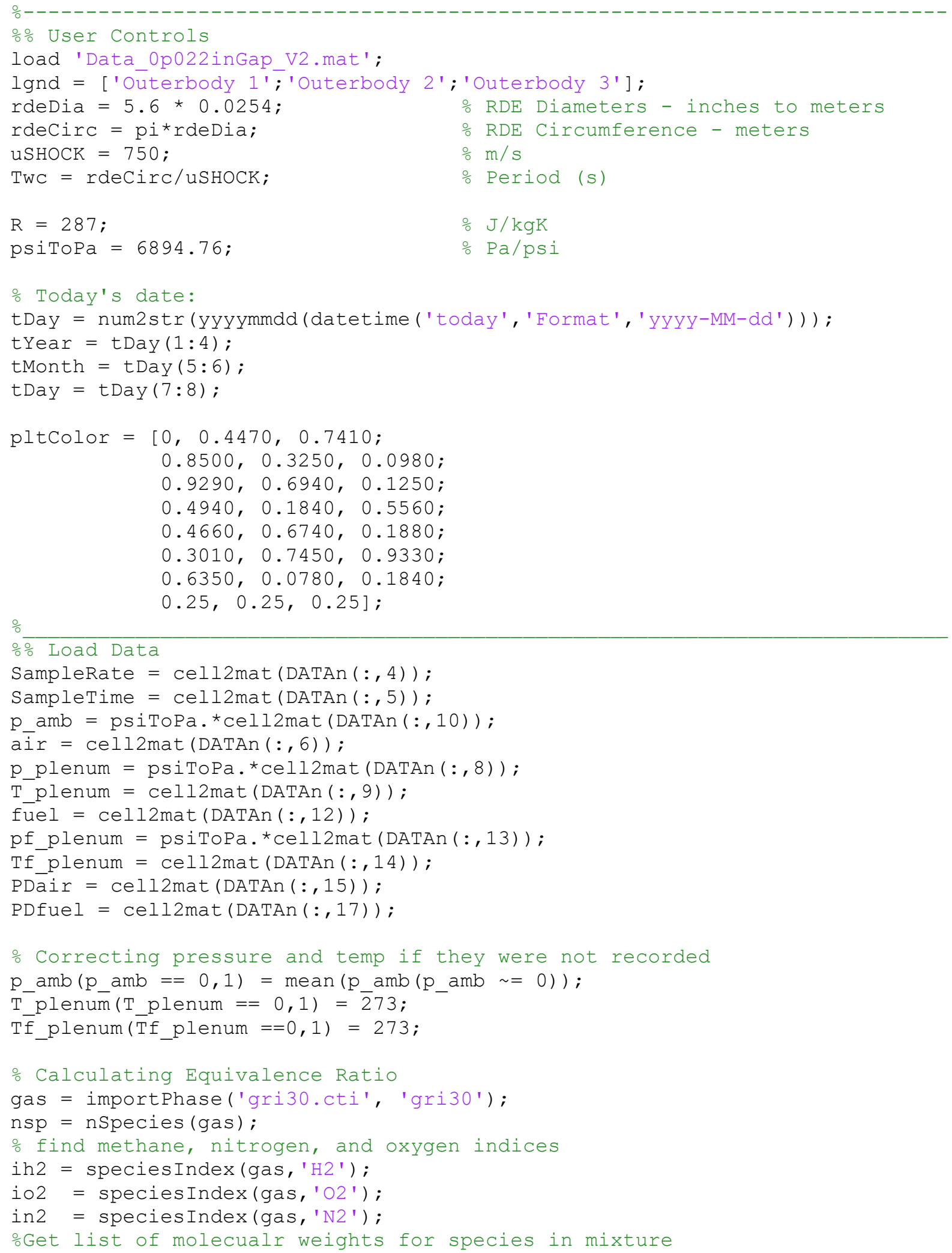




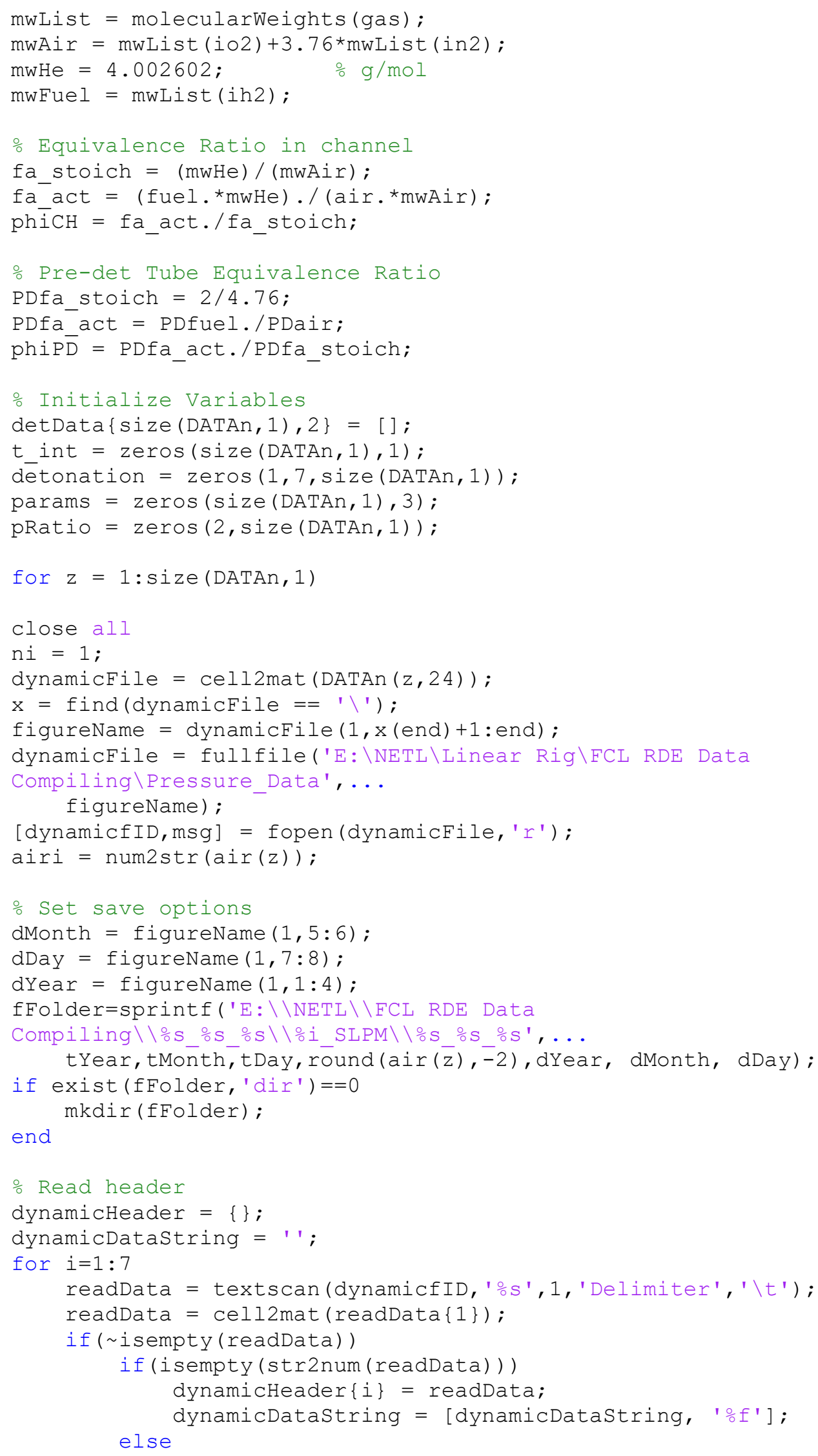




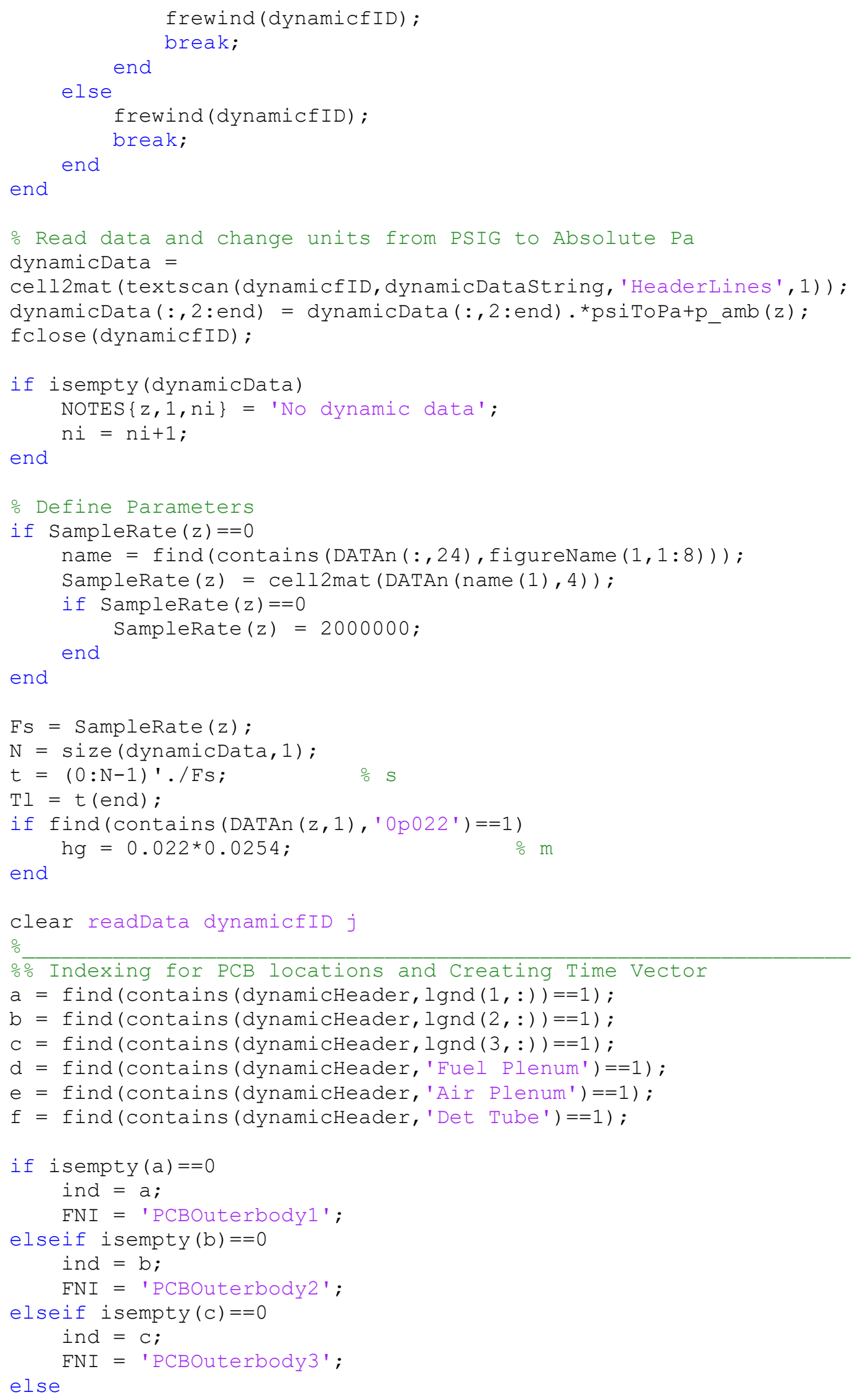




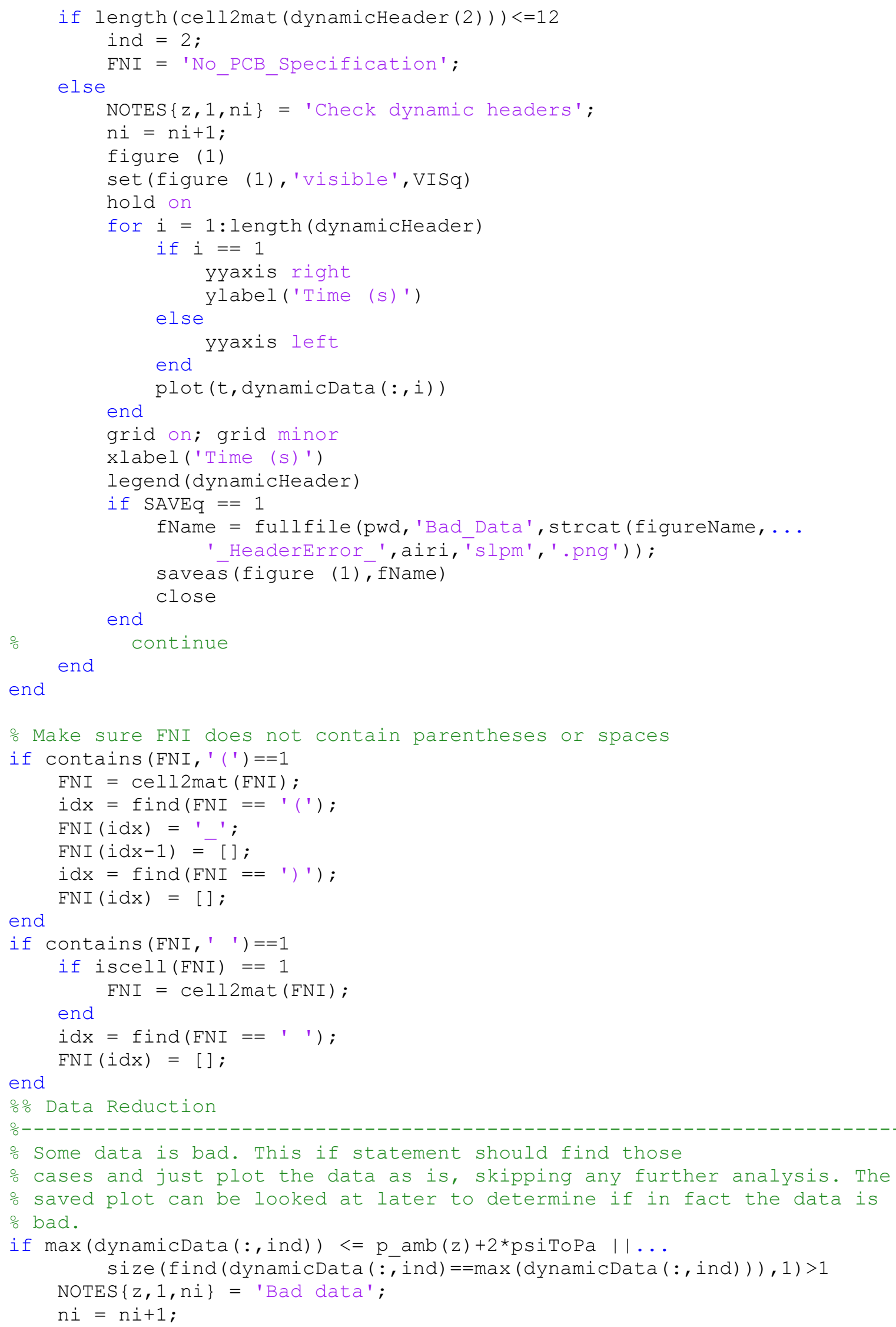




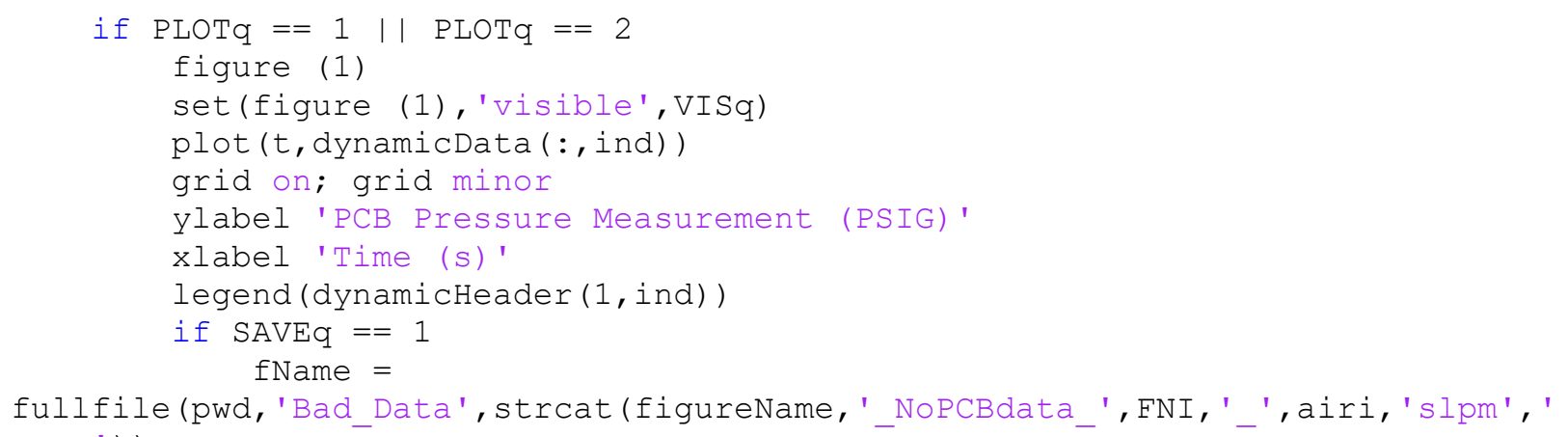




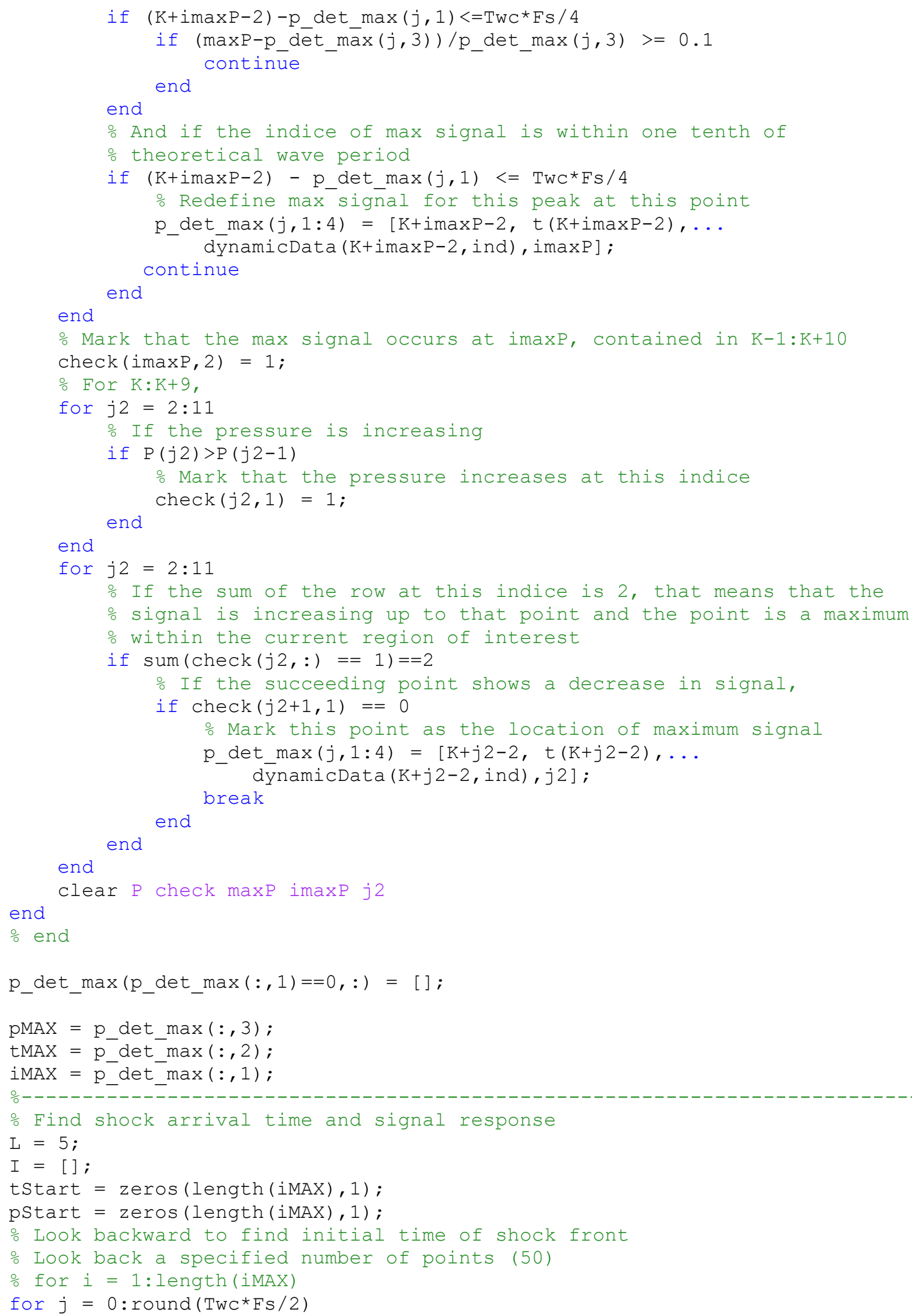




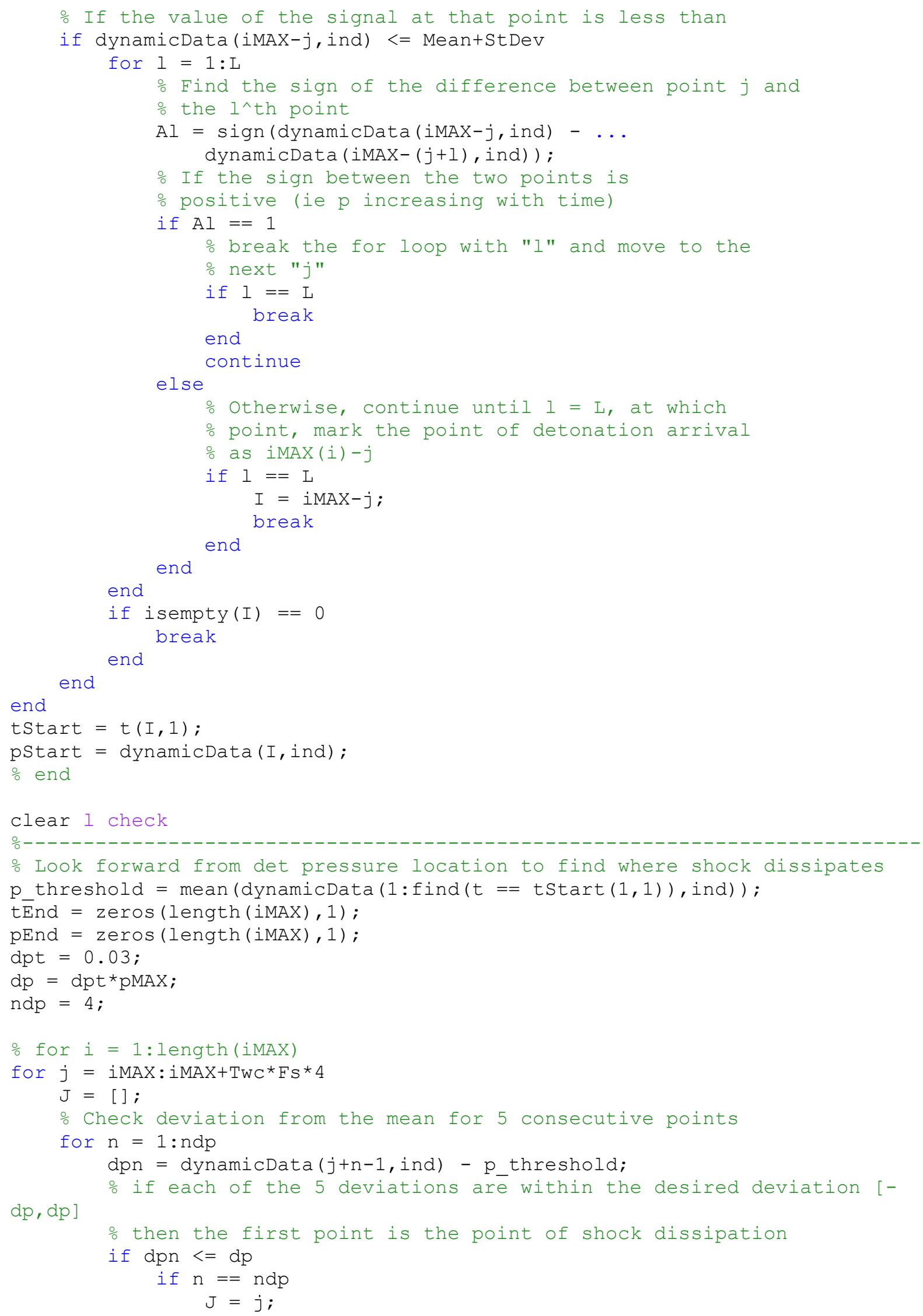




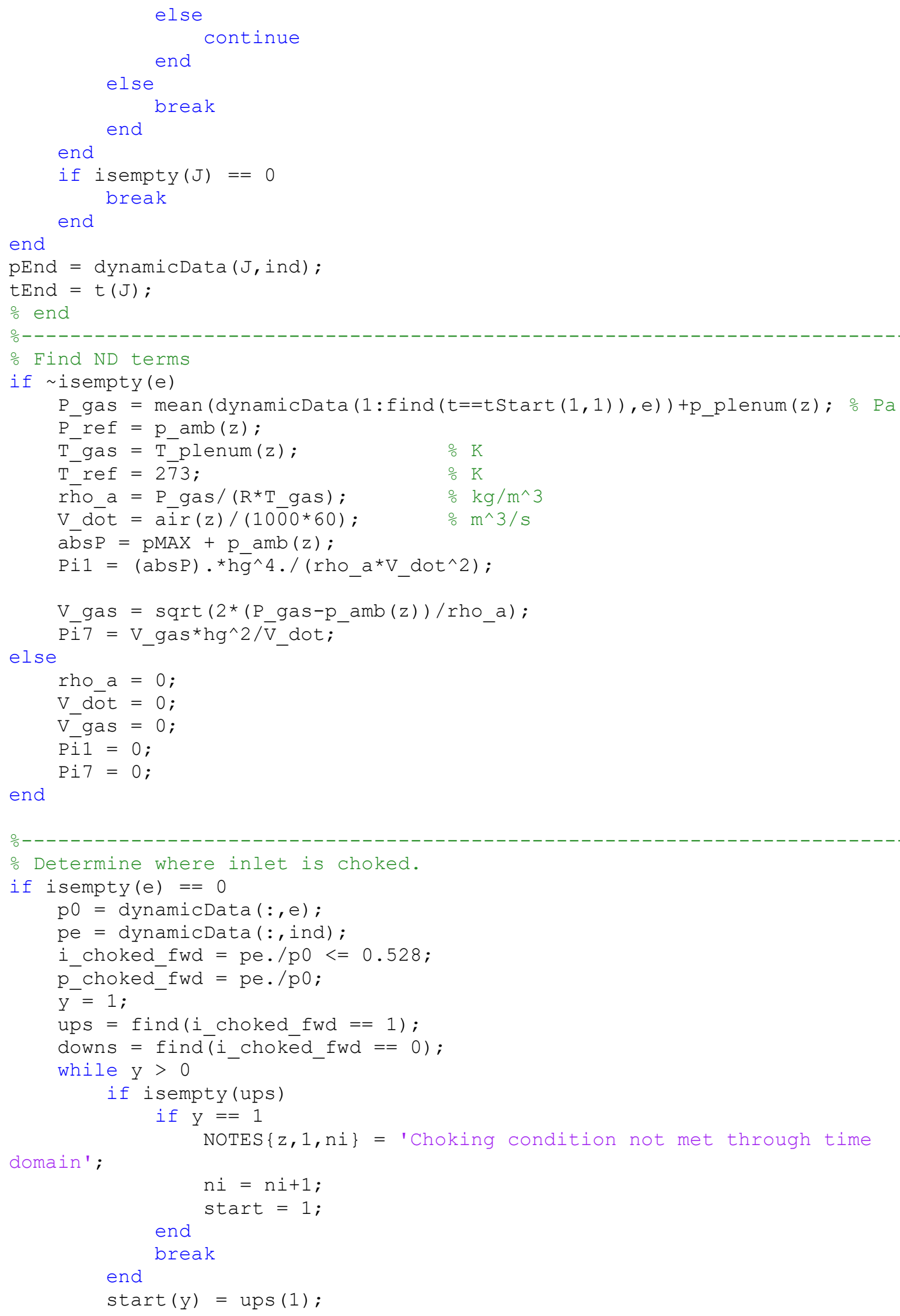




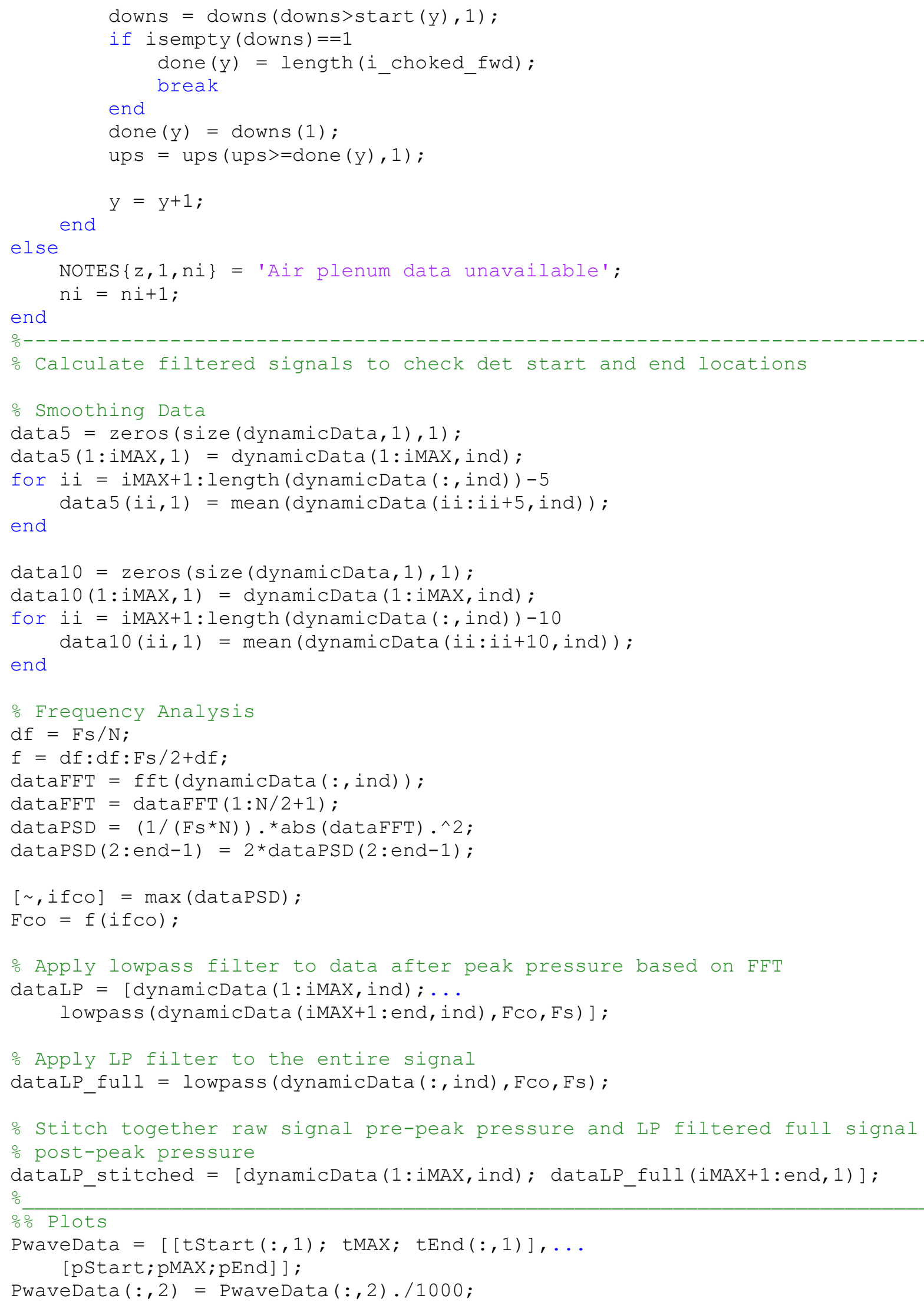




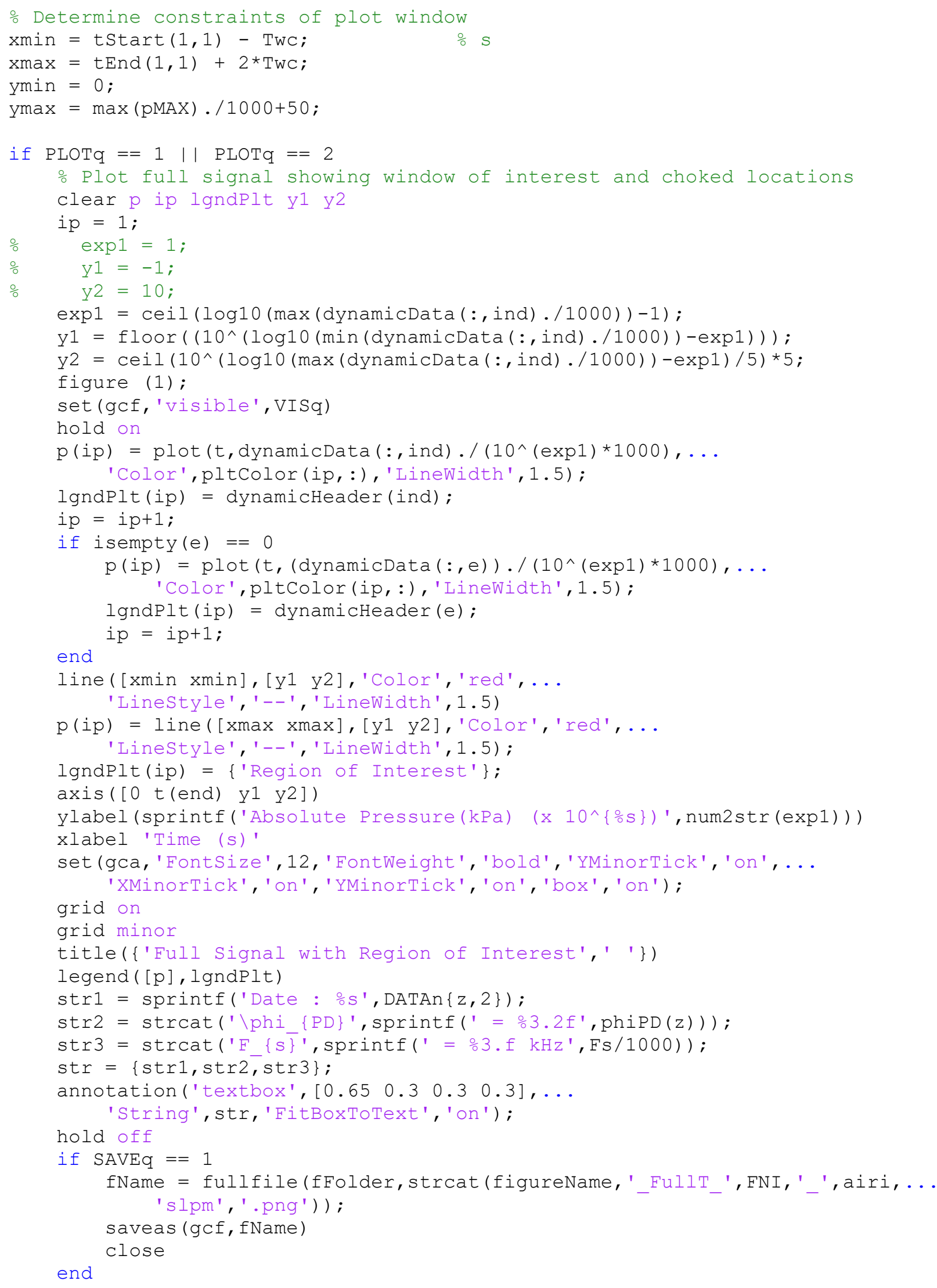


\% Plot window of interest with det start, p max, det dissipation,

o choked flow locations, and period of RDE

clear ip lgndPlt p

ip $=1$;

figure (2);

set (gcf, 'visible', VISq)

hold on

$\mathrm{p}(\mathrm{ip})=\mathrm{plot}\left(\mathrm{t} . \star 1000\right.$, dynamicData $(:$, ind $) . /\left(10^{\wedge}(\exp 1) \star 1000\right), \ldots$

'Color',pltColor(ip, :), 'LineWidth', 1.5);

lgndPlt $(\mathrm{ip})=$ dynamicHeader(ind) ;

$i p=i p+1$

$\mathrm{p}(\mathrm{ip})=\operatorname{plot}\left(\right.$ PwaveData $(1$ : length $($ PwaveData $) / 3,1) .{ }^{\star} 1000, \ldots$

PwaveData (1: length (PwaveData)/3,2) ./ (10^(exp1)),...

'sg', 'Linewidth', 3) ;

lgndPlt(ip) $=$ \{'Detonation Arrival'\};

$i p=i p+1$

$\mathrm{p}(\mathrm{ip})=$

plot (PwaveData (length (PwaveData)/3+1:2*length (PwaveData)/3, 1) . *1000, . .

PwaveData (length (PwaveData)/3+1:2*length (PwaveData)/3,2) ./(10^(exp1)), ... 'oc', 'LineWidth', 3);

lgndPlt $(i p)=\{$ 'Detonation Peak'\};

$i p=i p+1$

$\mathrm{p}(\mathrm{ip})=$

plot (PwaveData (2*length (PwaveData) /3+1: length (PwaveData), 1) . *1000, . .

PwaveData (2* length (PwaveData)/3+1: length (PwaveData), 2) ./ (10^ (exp1)), .. 'sr', 'LineWidth', 3);

lgndPlt $(i p)=\{$ 'Detonation Dissipation' $\}$;

$i p=i p+1$

tb = annotation('textbox', [ $\left.\begin{array}{lllll}0.65 & 0.1 & 0.3 & 0.3\end{array}\right], \ldots$

'String', str, 'FitBoxToText', 'on' ', 'BackgroundColor', 'white') ;

title(\{'Detonation Characteristics within Region of Interest',' ' \})

hold off

if $\mathrm{SAVEq}==1$

fName = fullfile(fFolder, strcat(figureName, '_ROI_',FNI, '_', airi,...

'slpm', '.png'));

saveas (gcf, fName)

close

end

end

if $\mathrm{PLOTq}==2$

\% Plot shock analytics from raw signal calculations over 5 pt averaged

\% filtered data to show that methods produce accurate enough

\% evaluations of shock start, end, and peak. Plot results of applying

various filters to raw signal from moment

\% of peak pressure onward to show how closely matching each is

clear ip p lgndPlt

ip $=1$;

figure (3);

set(gcf, 'visible', VISq);

hold on

$\mathrm{p}(\mathrm{ip})=\operatorname{plot}\left(\mathrm{t} .{ }^{*} 1000\right.$, dynamicData $(:$, ind $) . /\left(10^{\wedge}(\exp 1) \star 1000\right), \ldots$

'Color', pltColor(ip, :), 'Linewidth', 1.5);

$\operatorname{lgndPlt}(\mathrm{ip})=$ dynamicHeader $(\mathrm{ind})$;

$i p=i p+1$; 


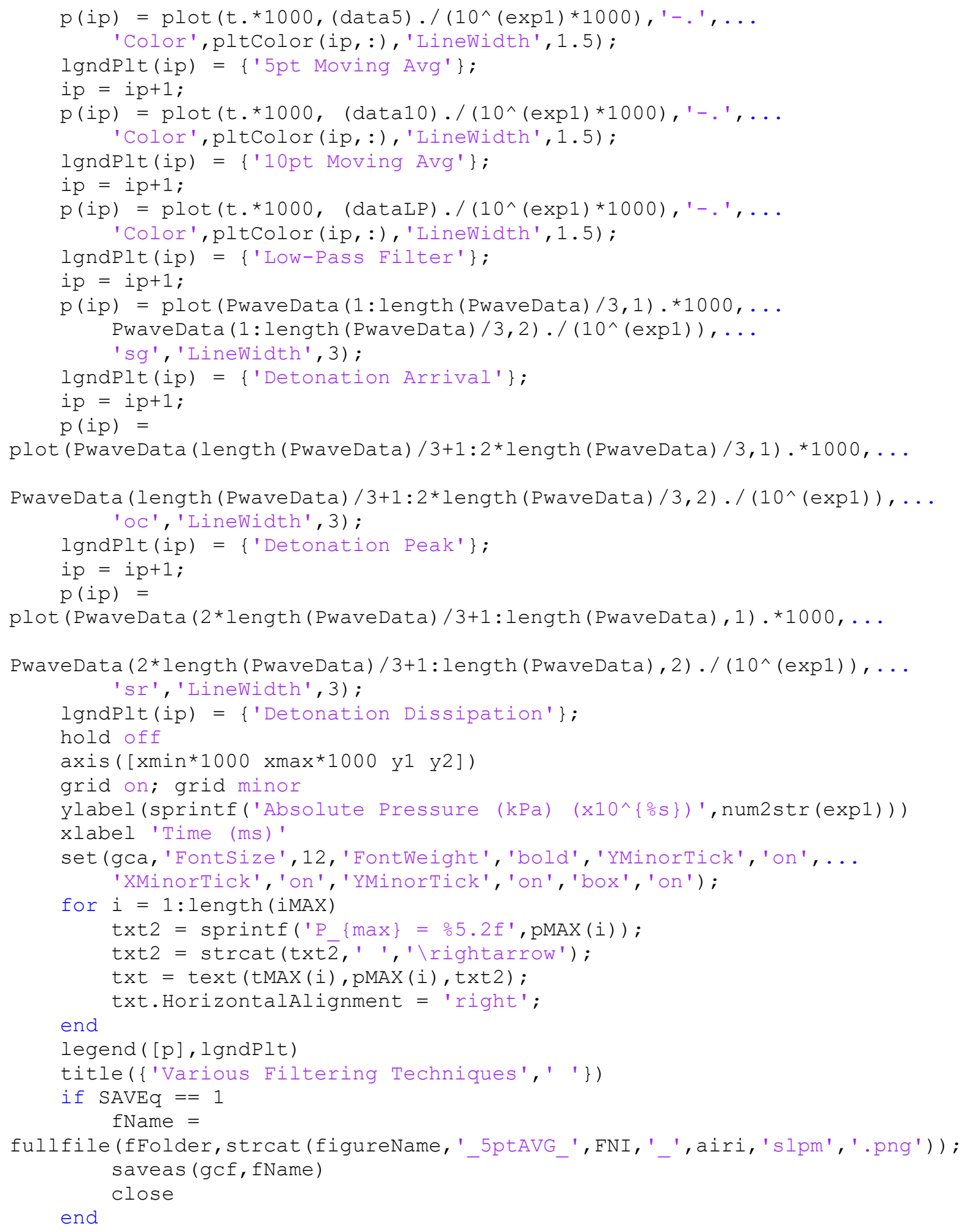




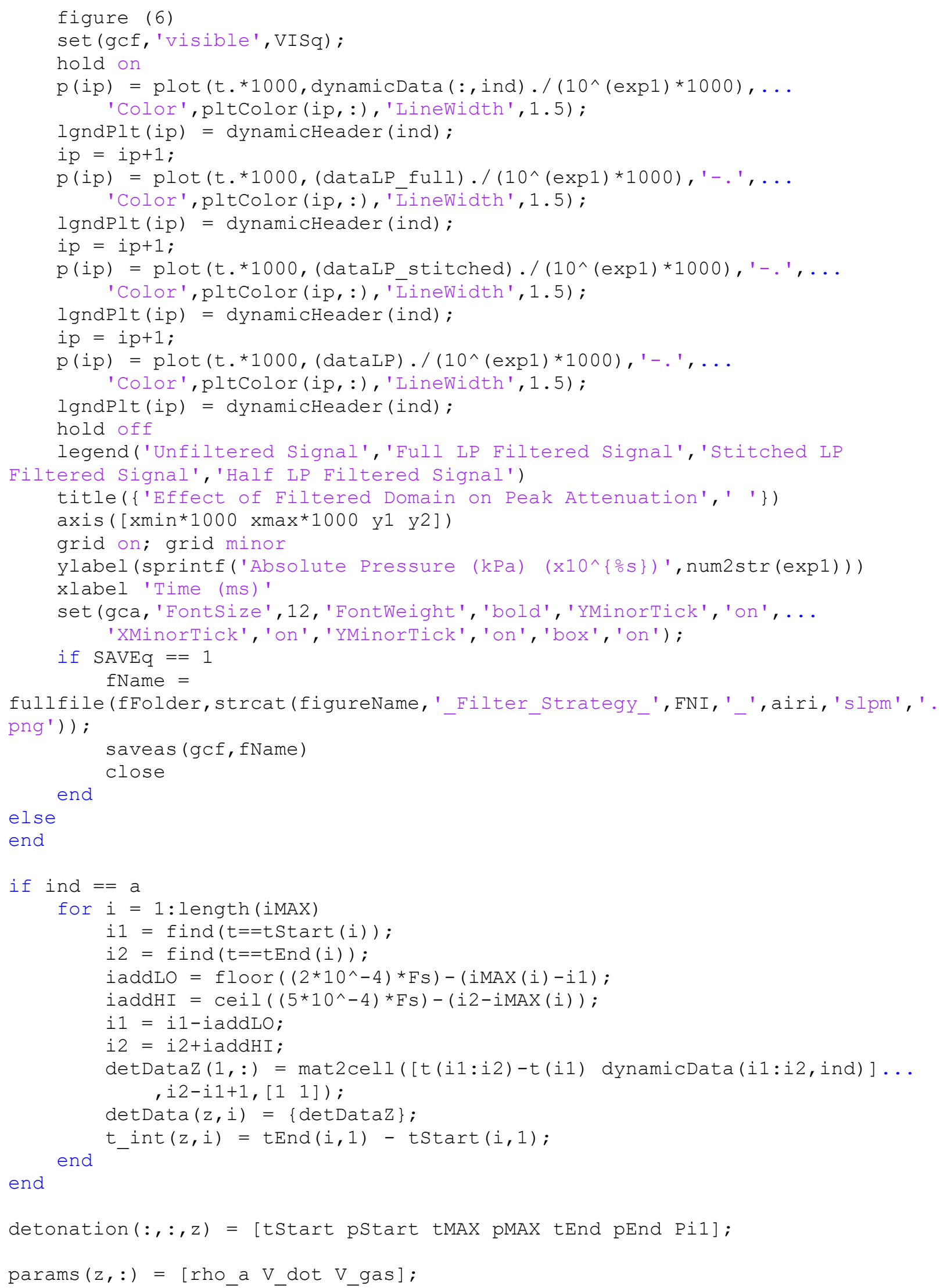




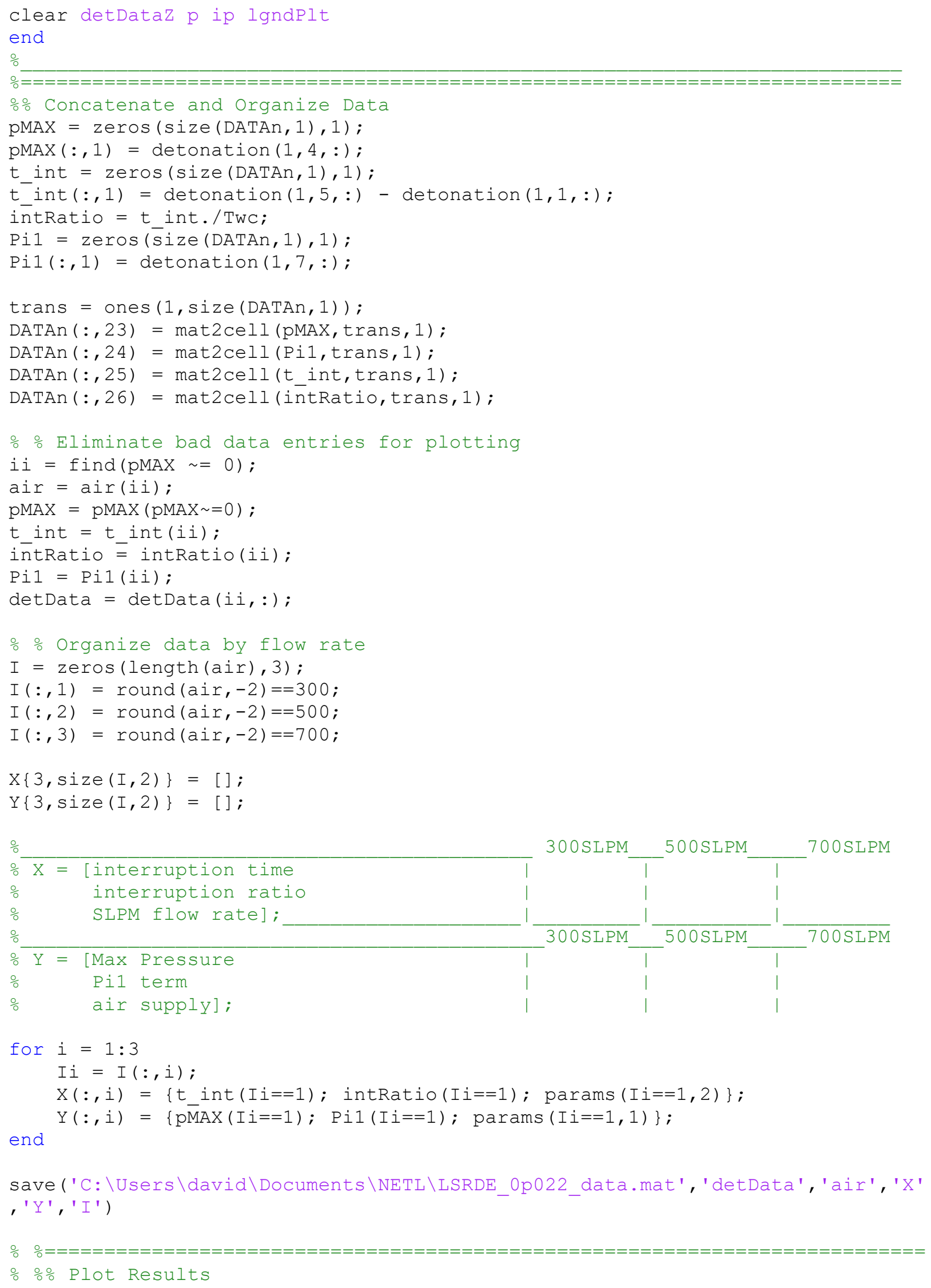




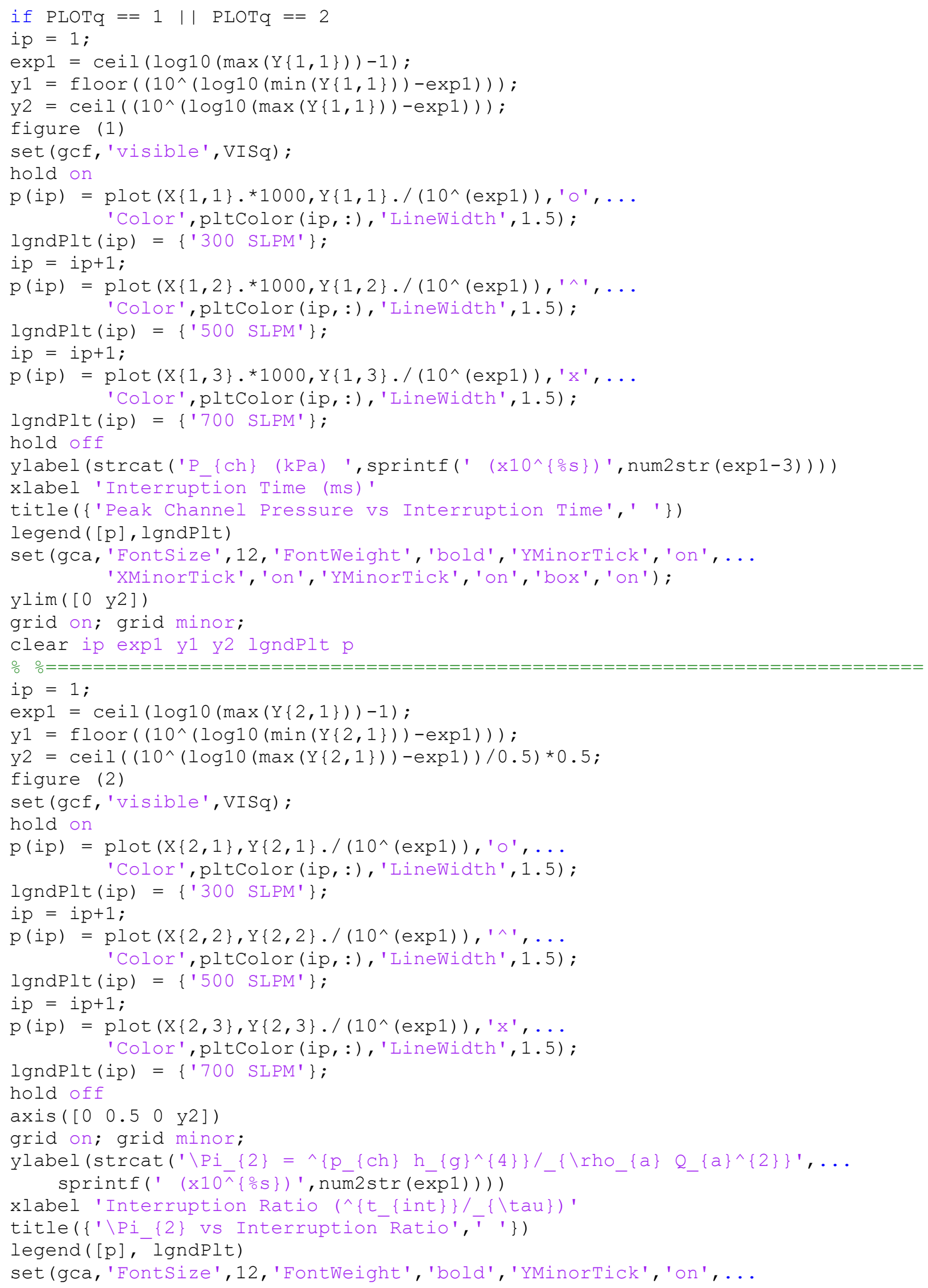


'XMinorTick', 'on' ', 'YMinorTick', 'on' , 'box' , 'on' );

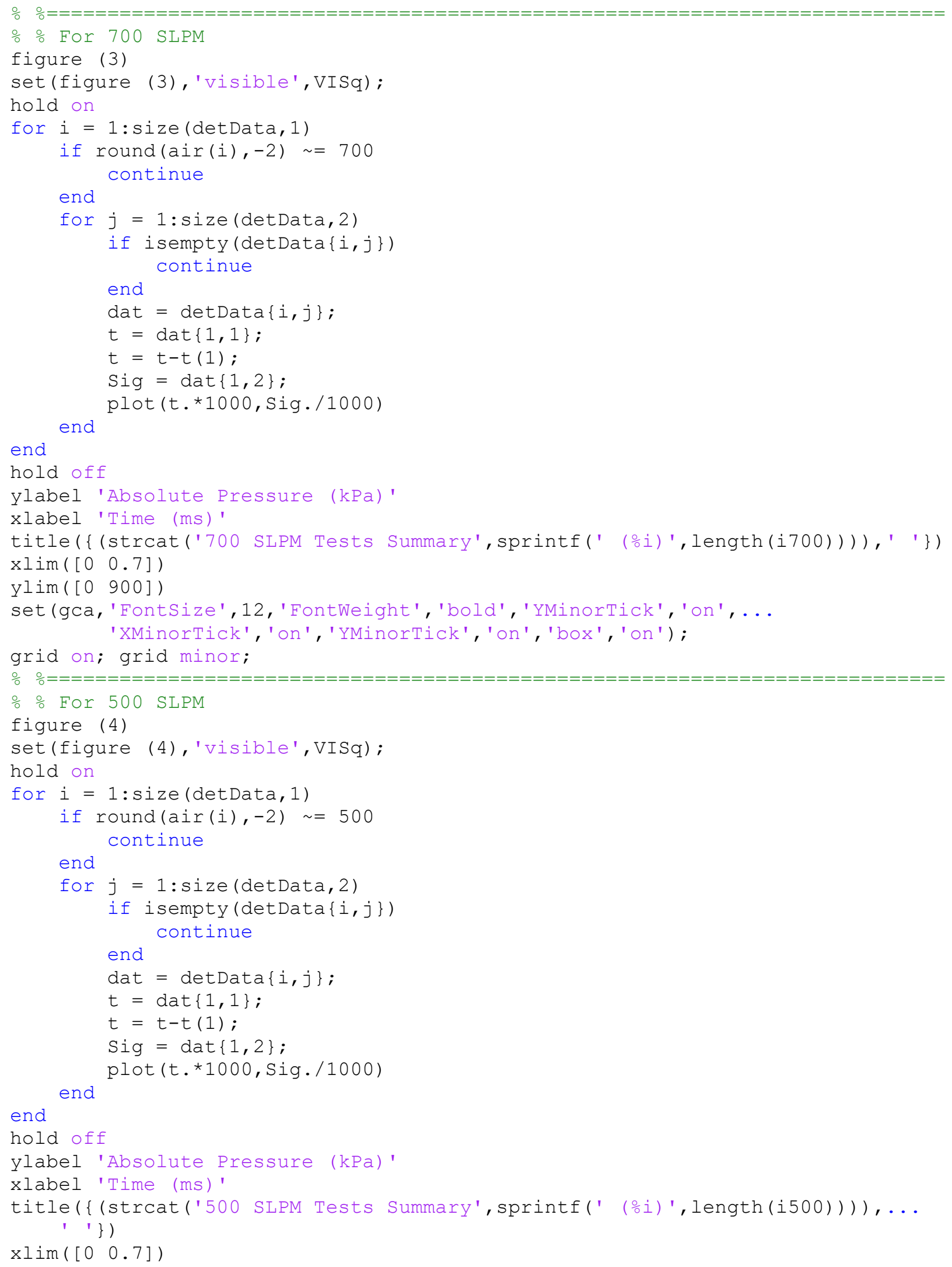




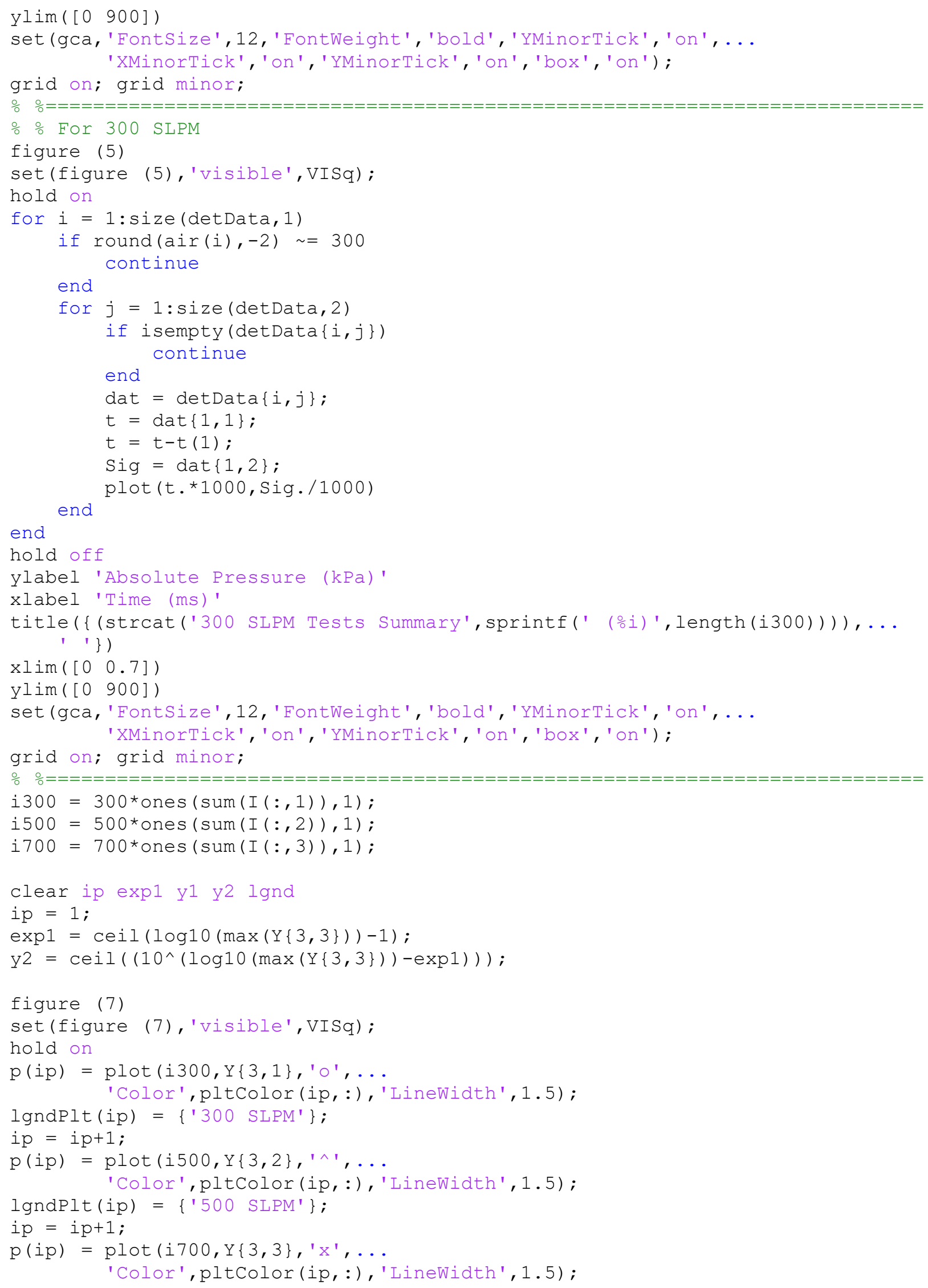




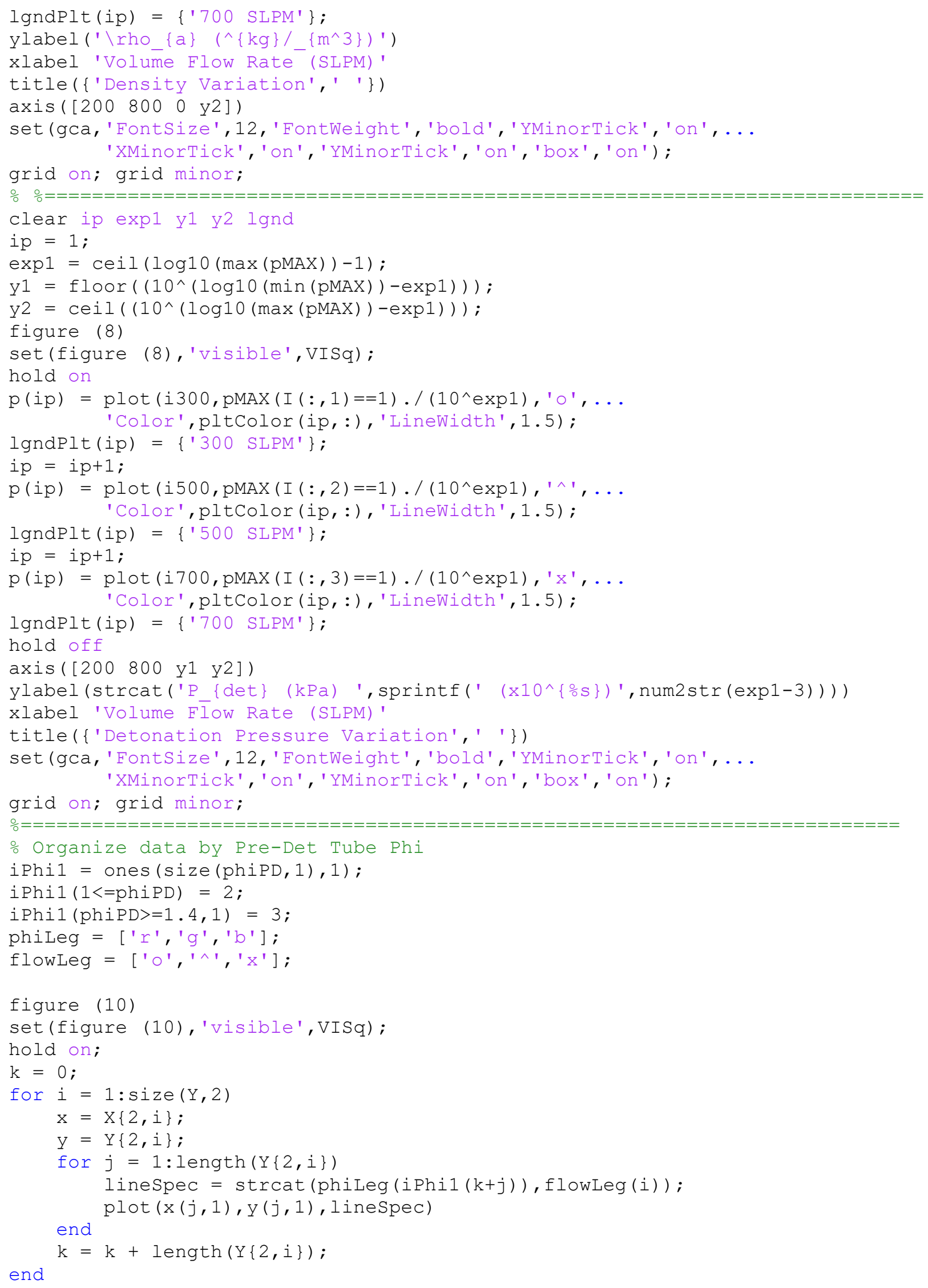




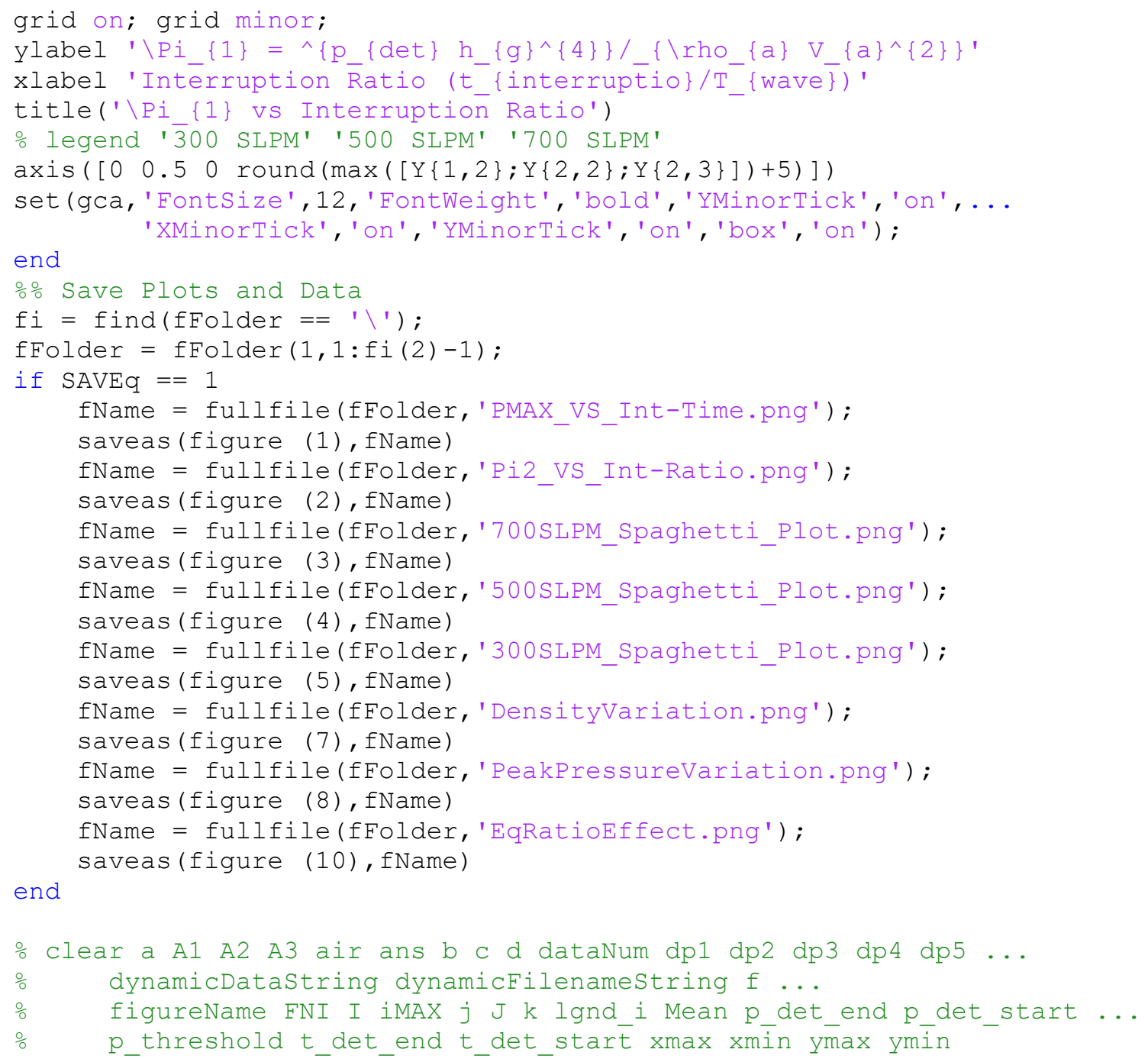




\subsection{Appendix D: LSRDE MATLAB Code}

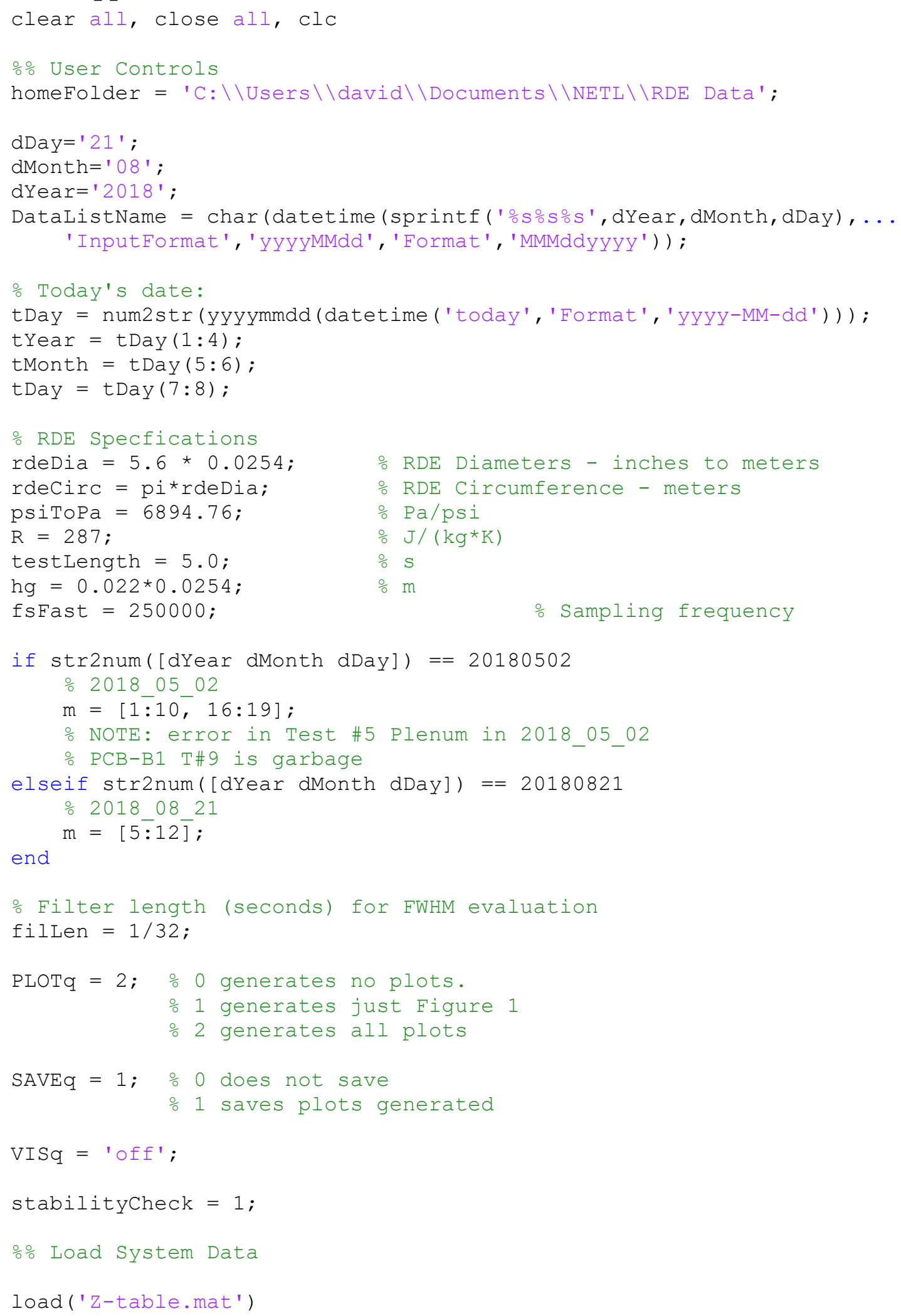




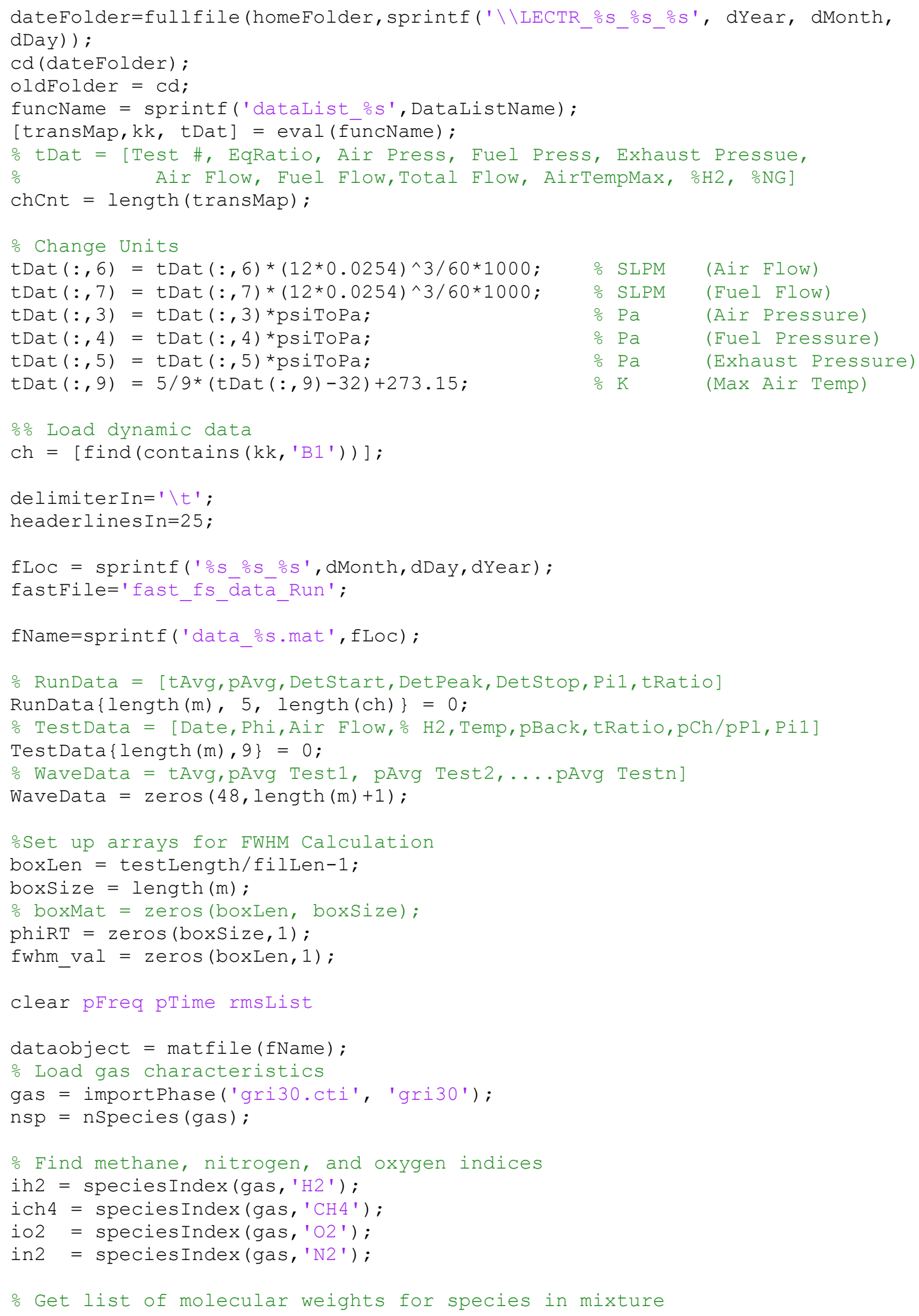




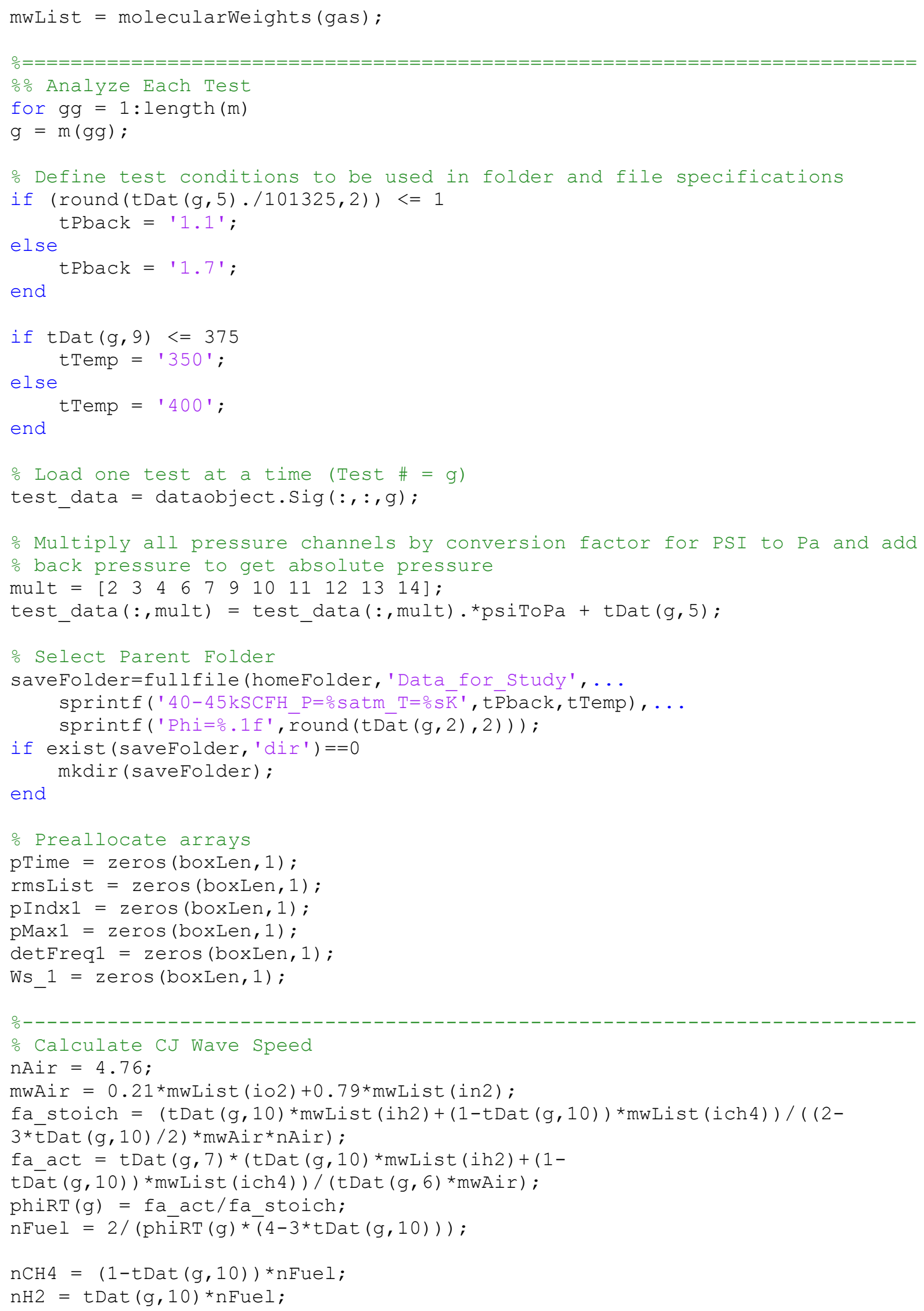




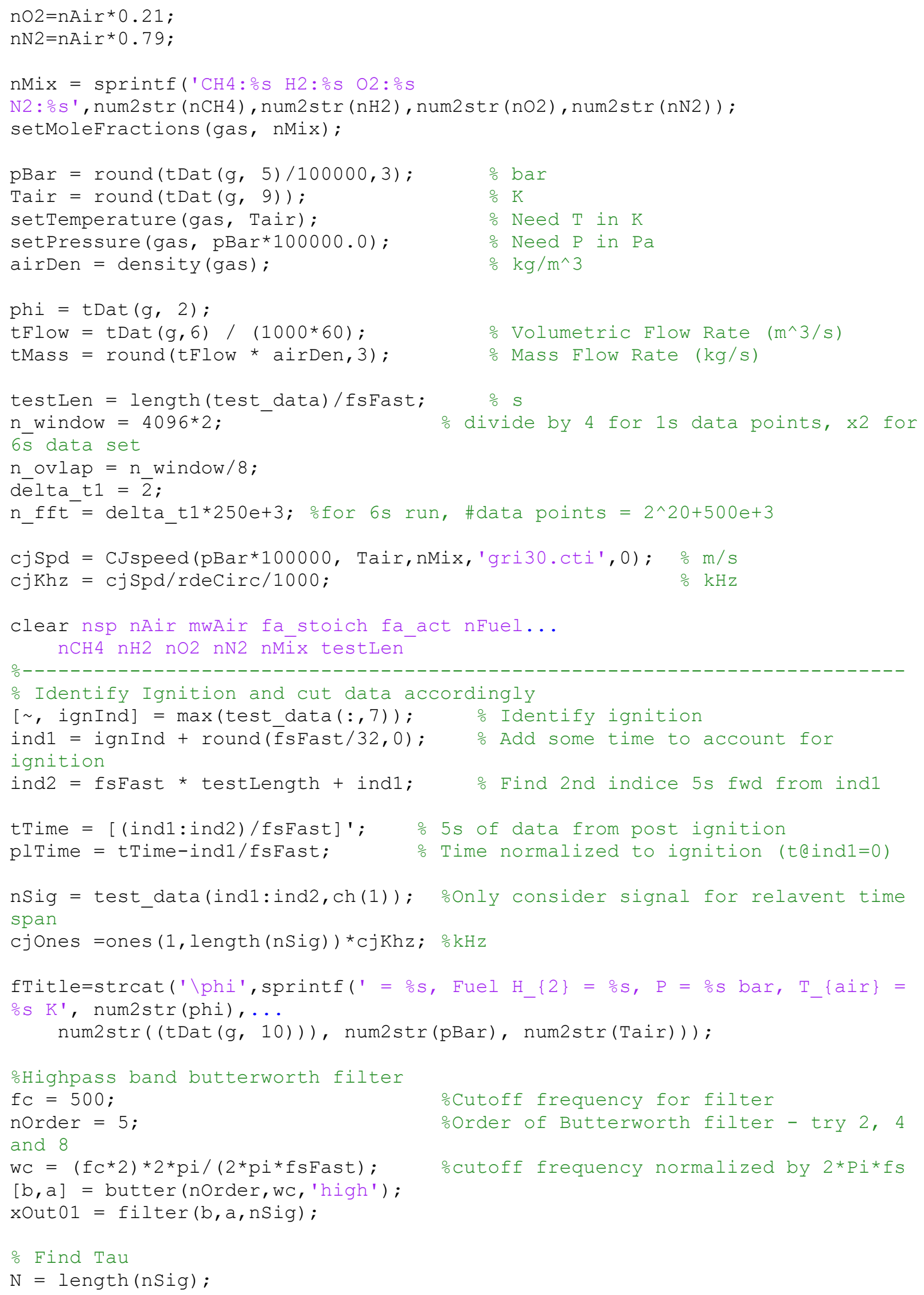




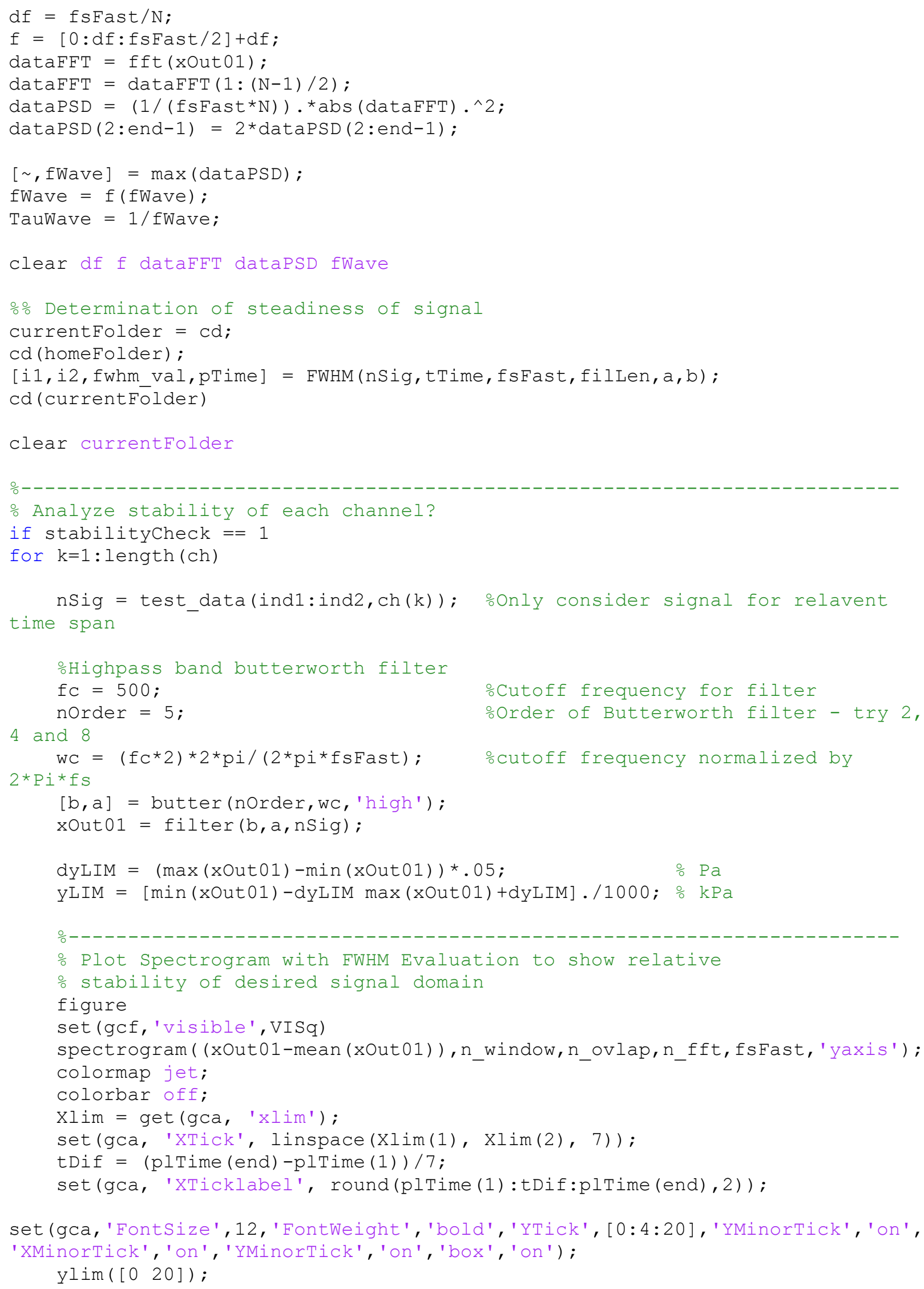




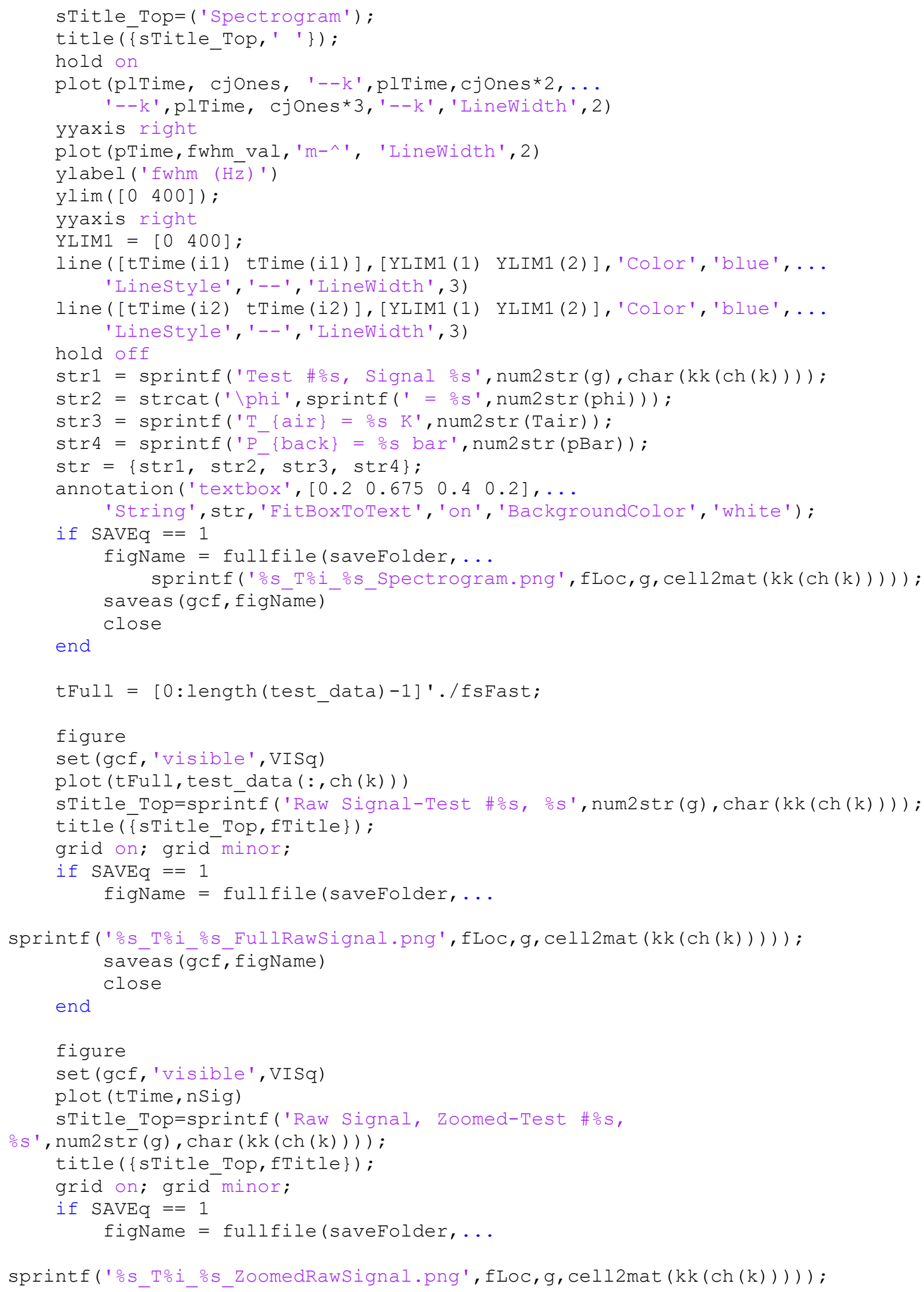




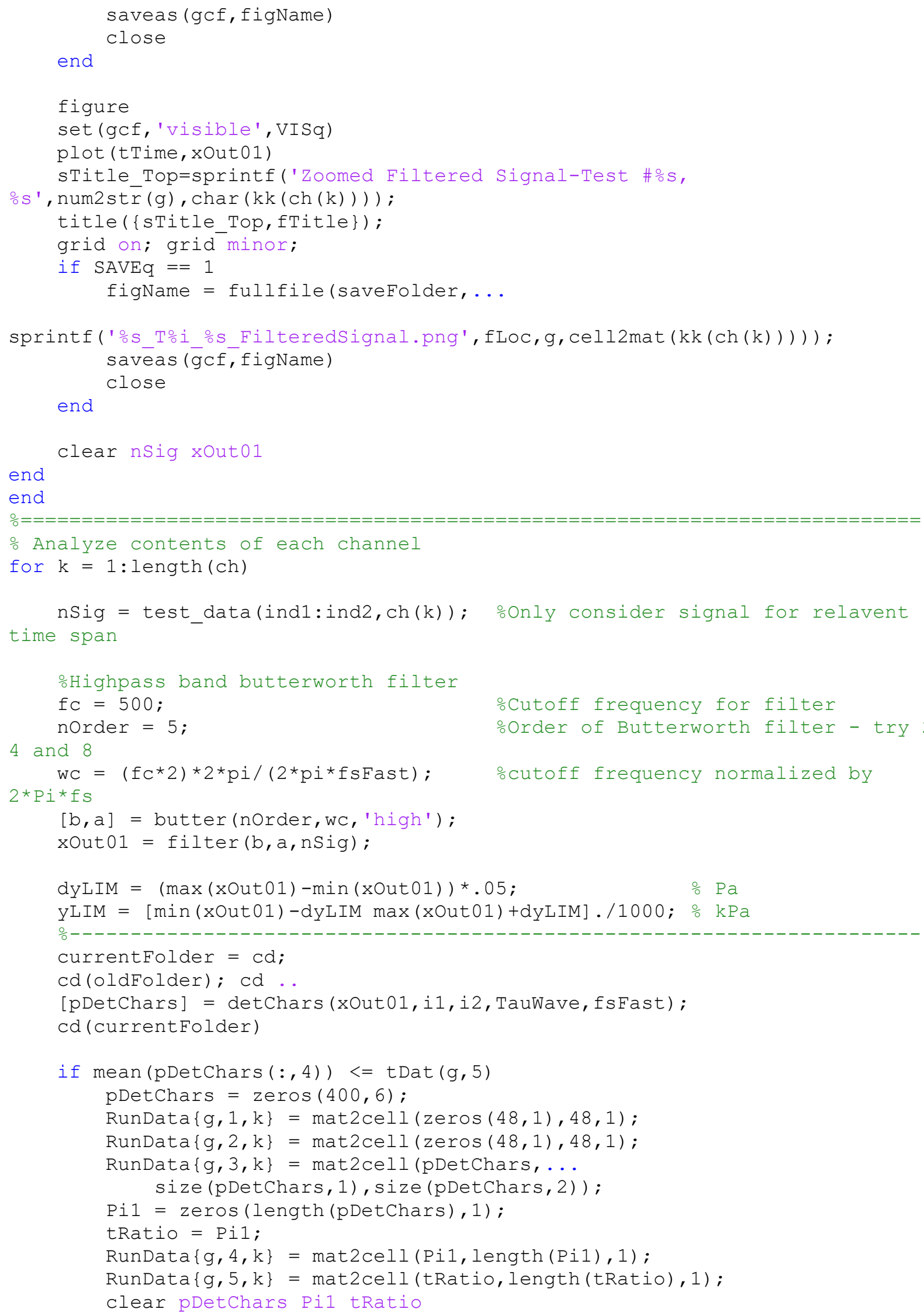




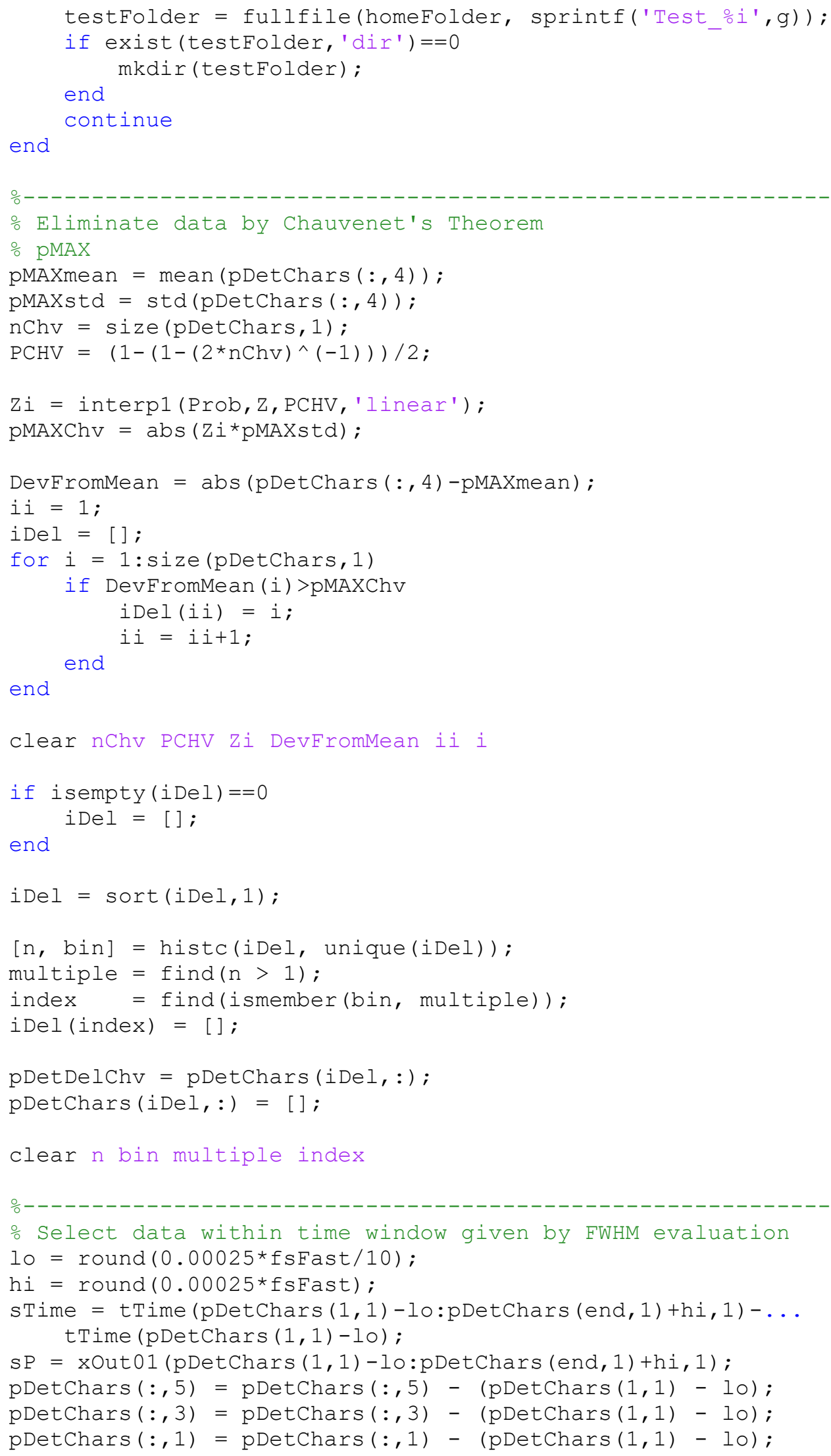




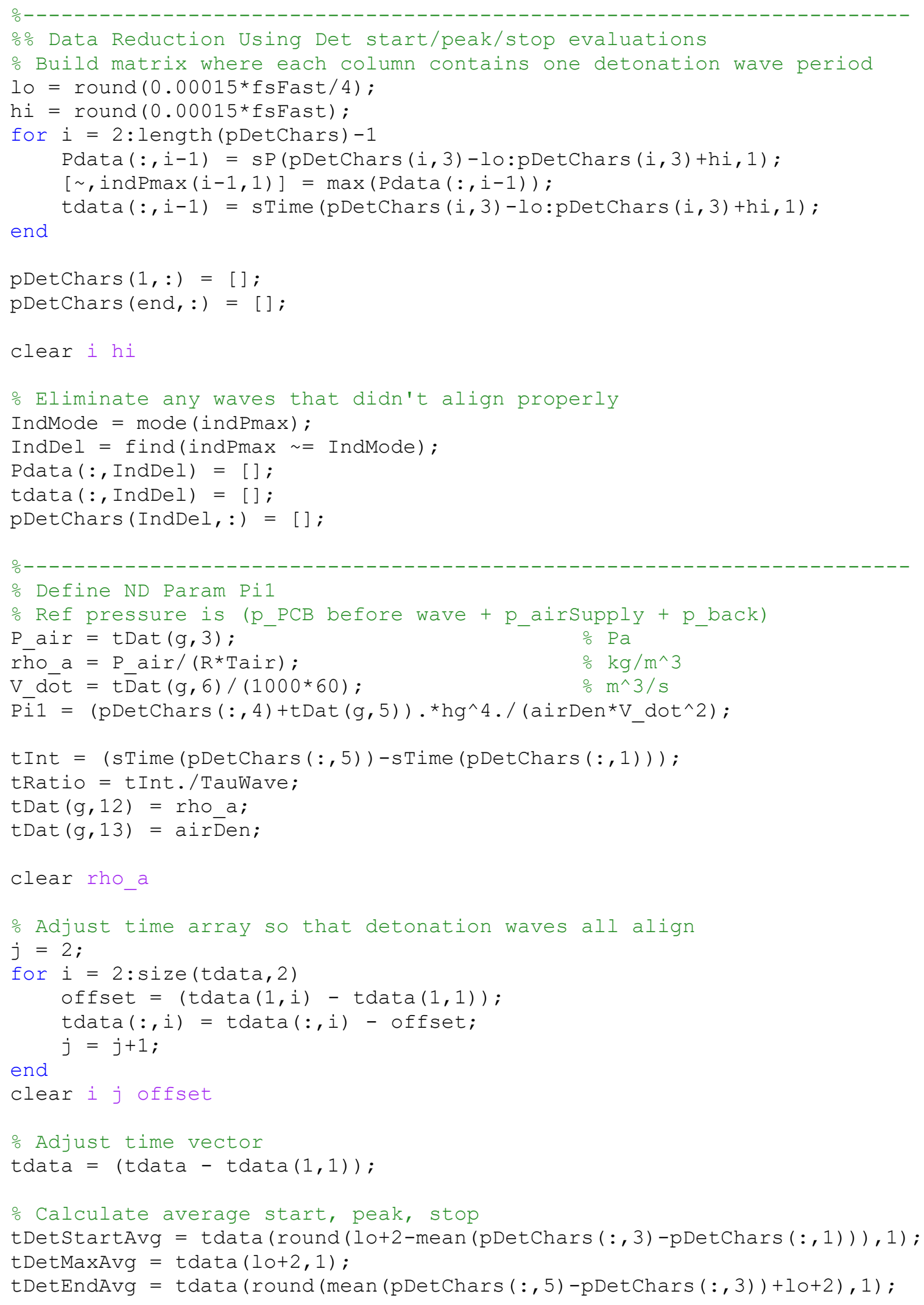




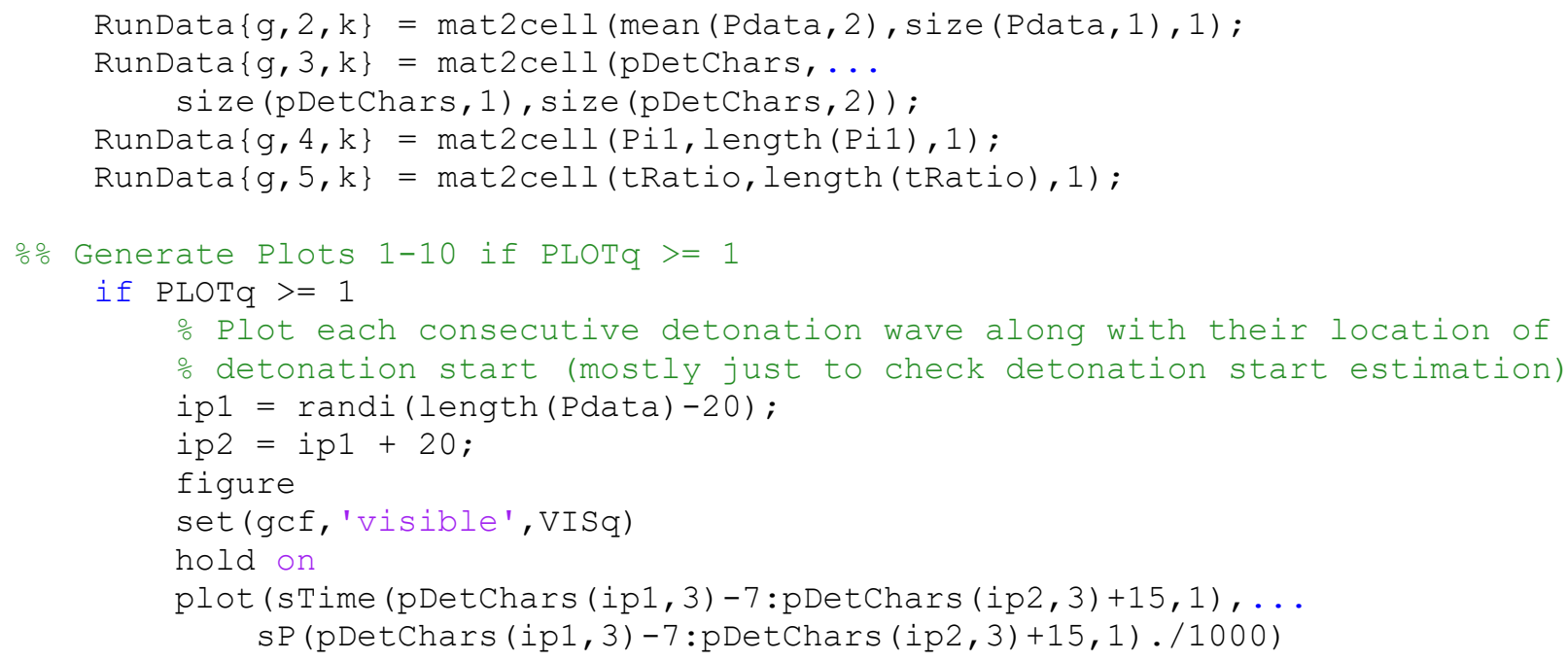




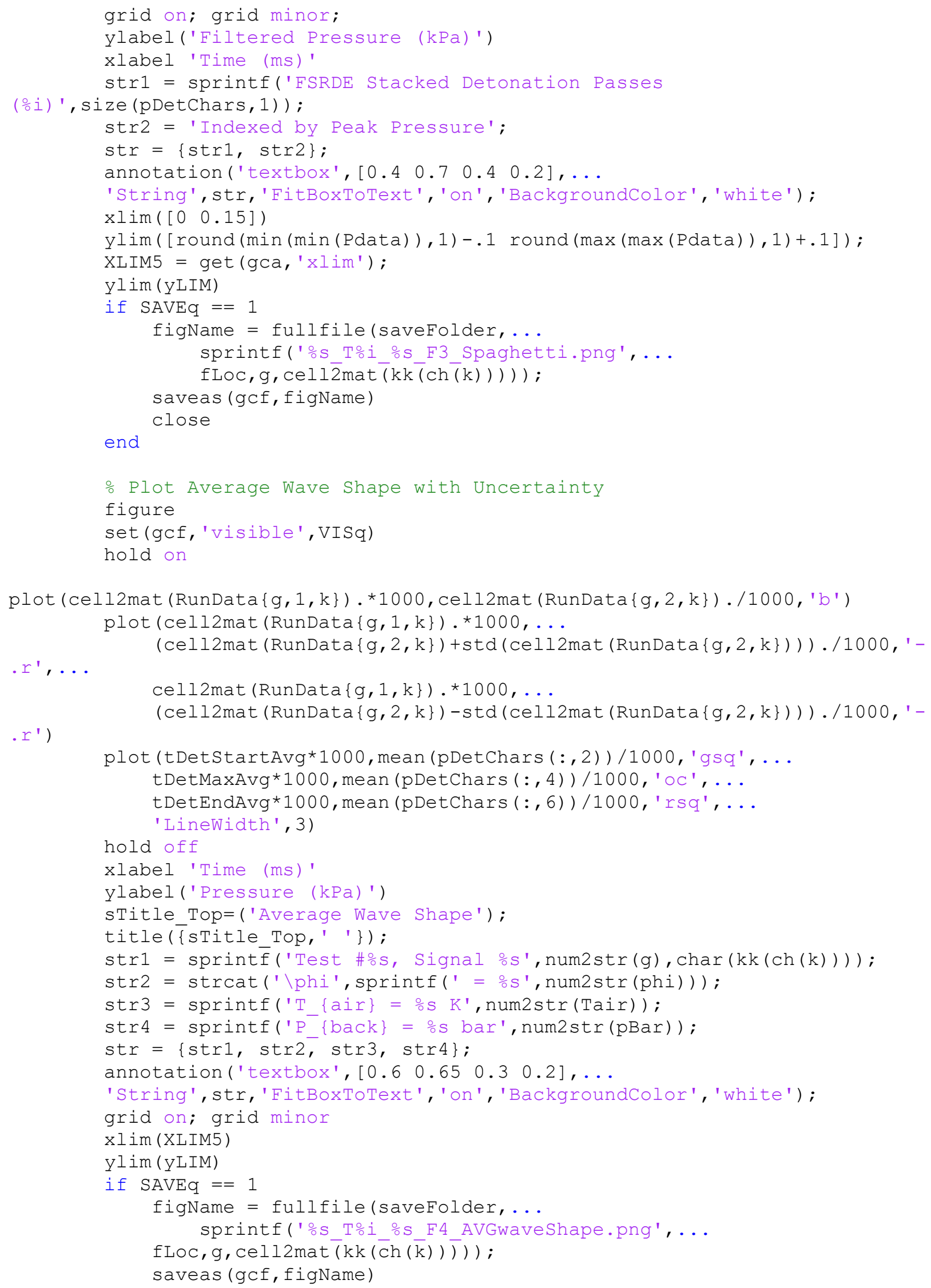




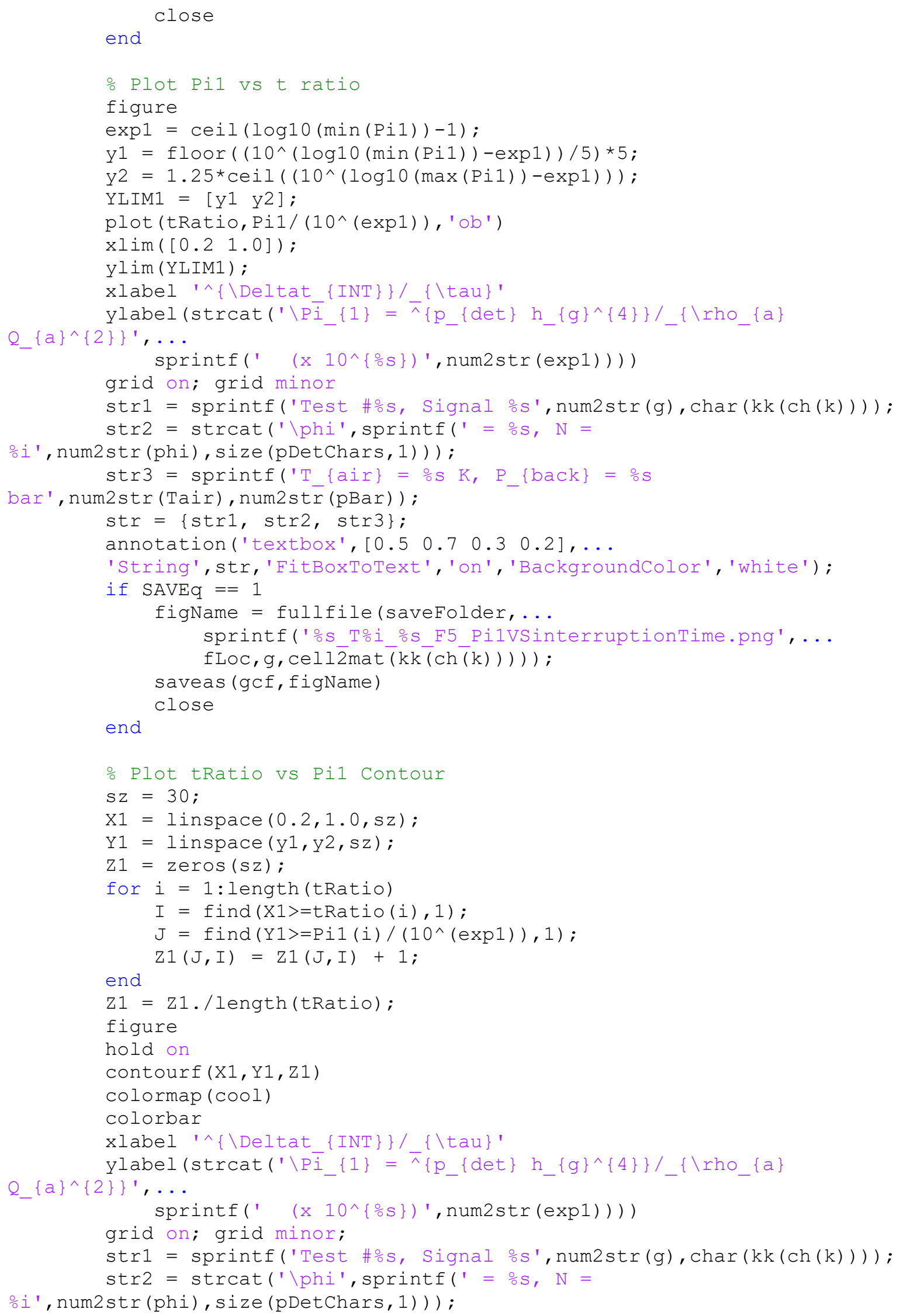




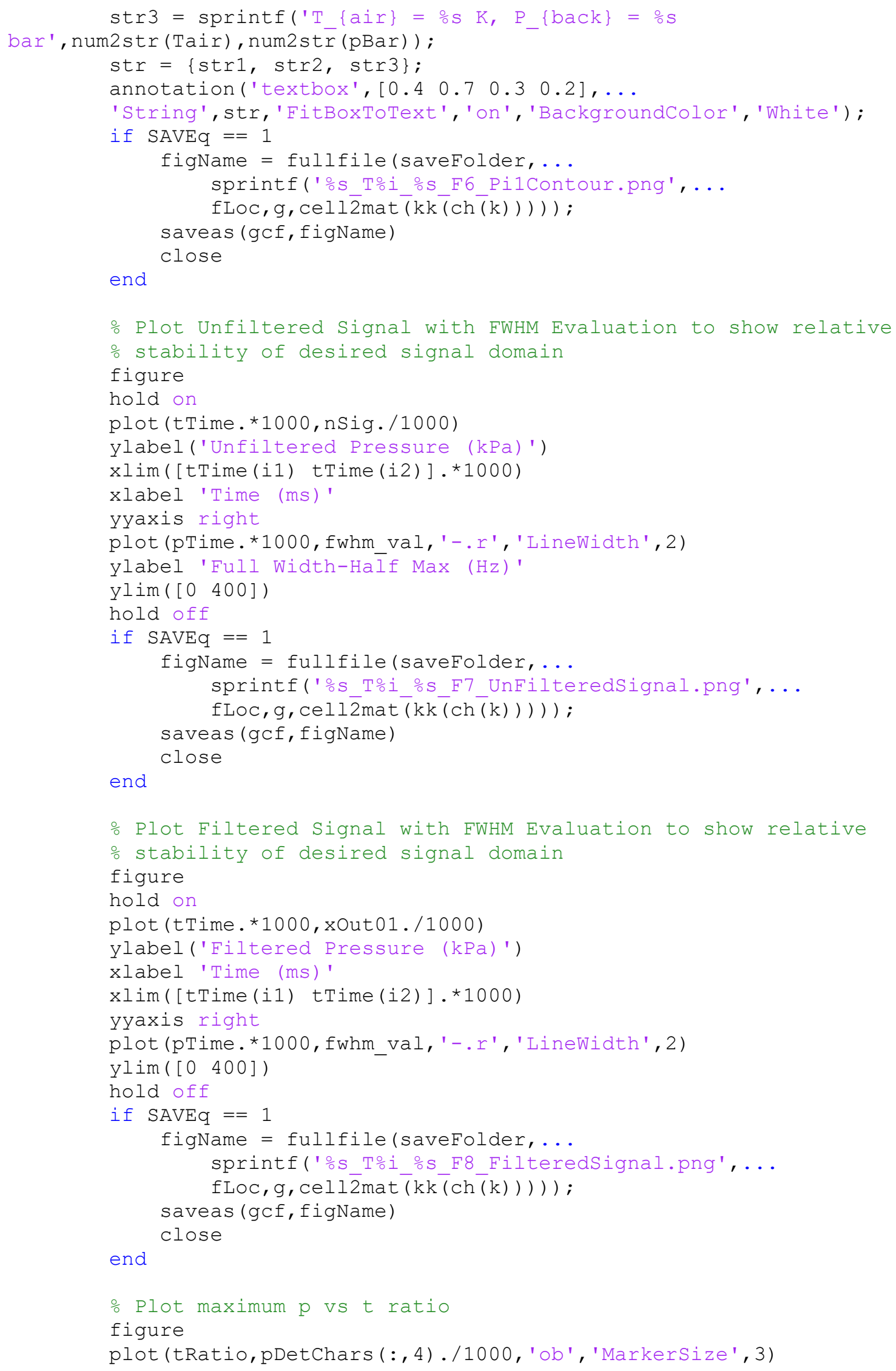




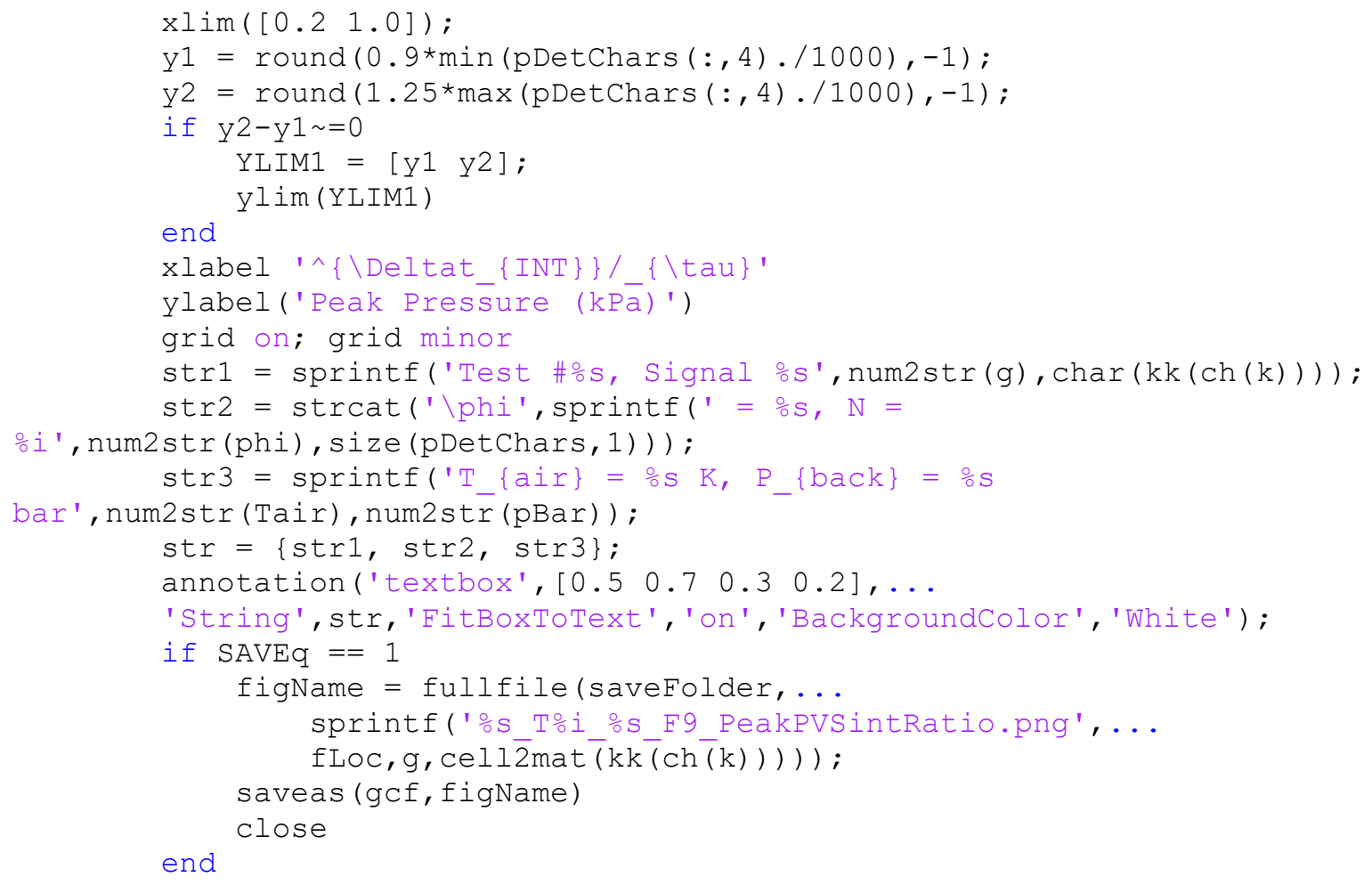

end

\% tDat $=$ [Test \#, EqRatio, Air Press, Fuel Press, Exhaust Pressue,

․ Air Flow, Fuel Flow, Total Flow, AirTempMax, H2, NG]

TestData $(g, 1)=\{\operatorname{str} 2$ double $([$ dYear dMonth dDay $])\}$;

TestData $(g, 2)=\{\operatorname{Dat}(g, 2)\} ; \quad \div \operatorname{Phi}$

TestData $(g, 3)=\{\operatorname{tDat}(g, 6)\} ; \quad \div$ Air Flow (SLPM)

TestData $(9,4)=\{\operatorname{tDat}(9,10)\} ; \quad$ PCt H2

TestData $(9,5)=\{\operatorname{tDat}(9,9)\} ; \quad \frac{\circ}{0} \operatorname{Temp}(\mathrm{K})$

TestData $(9,6)=\{\operatorname{tDat}(9,5)\} ; \quad \therefore$ Back Pressure (Pa)

TestData $(9,7)=\operatorname{RunData}\{9,5,1\} ; \quad \div$ tRatio 


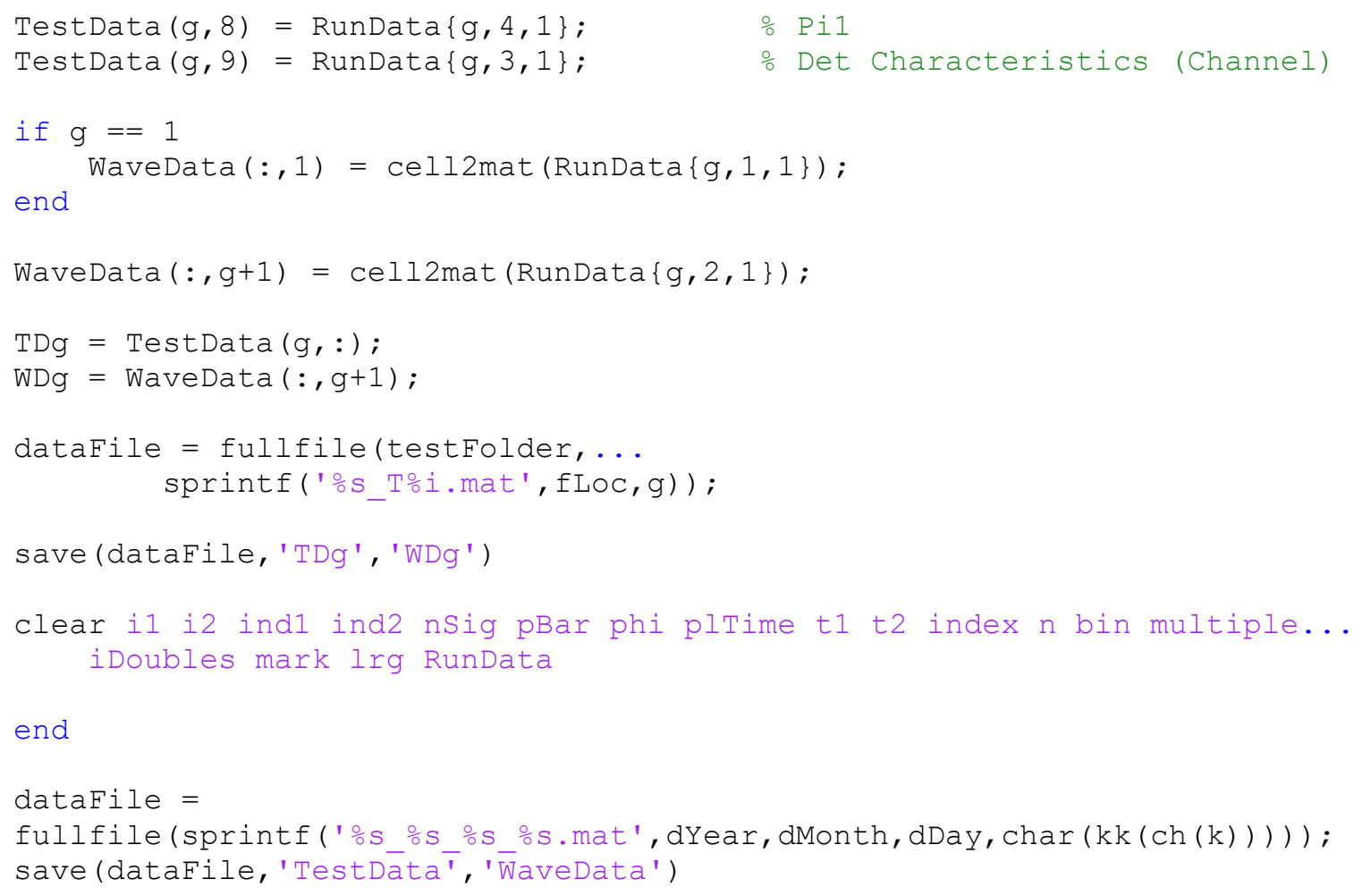




\subsection{Appendix E: LSRDE Data Summary Plotting}

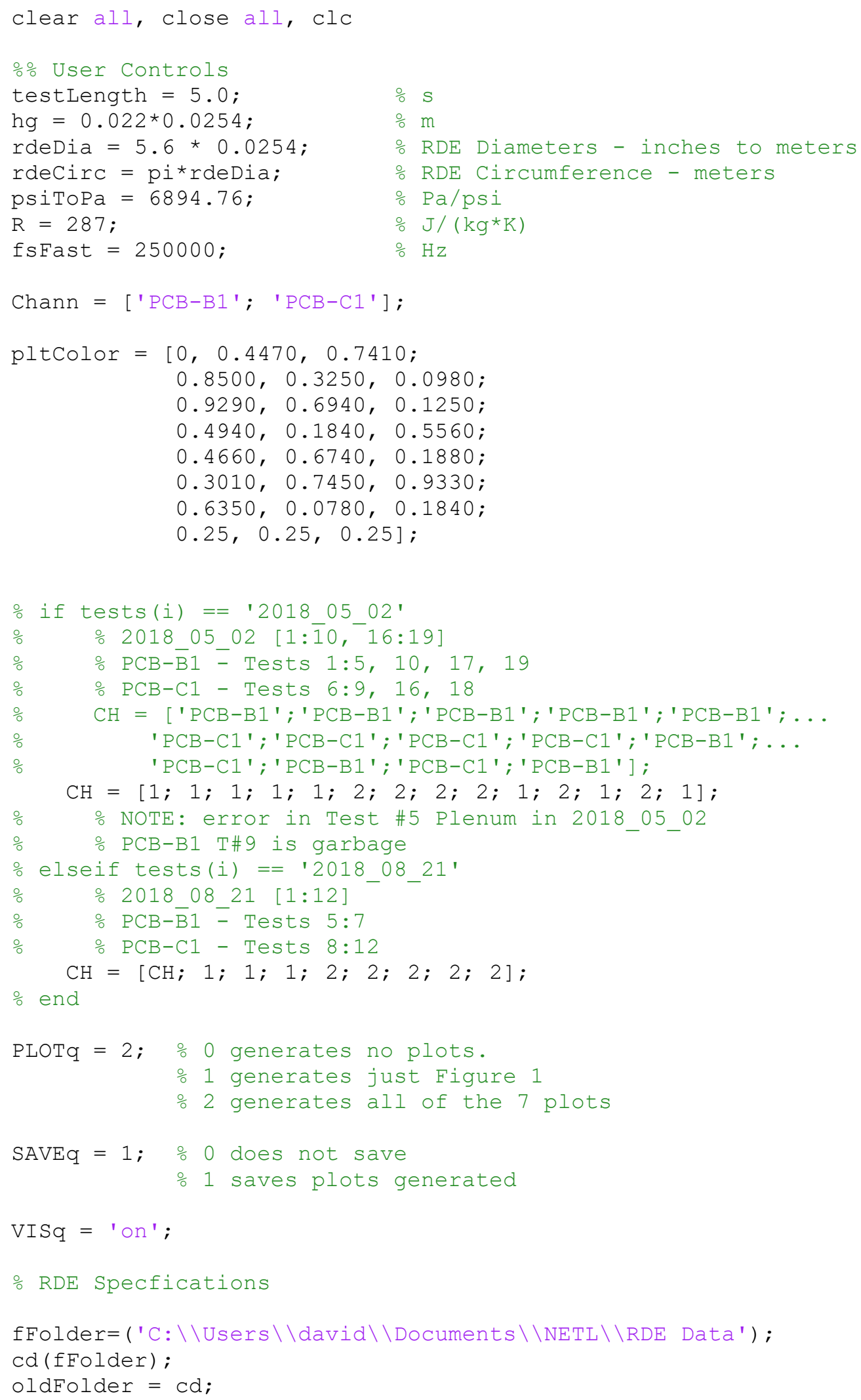




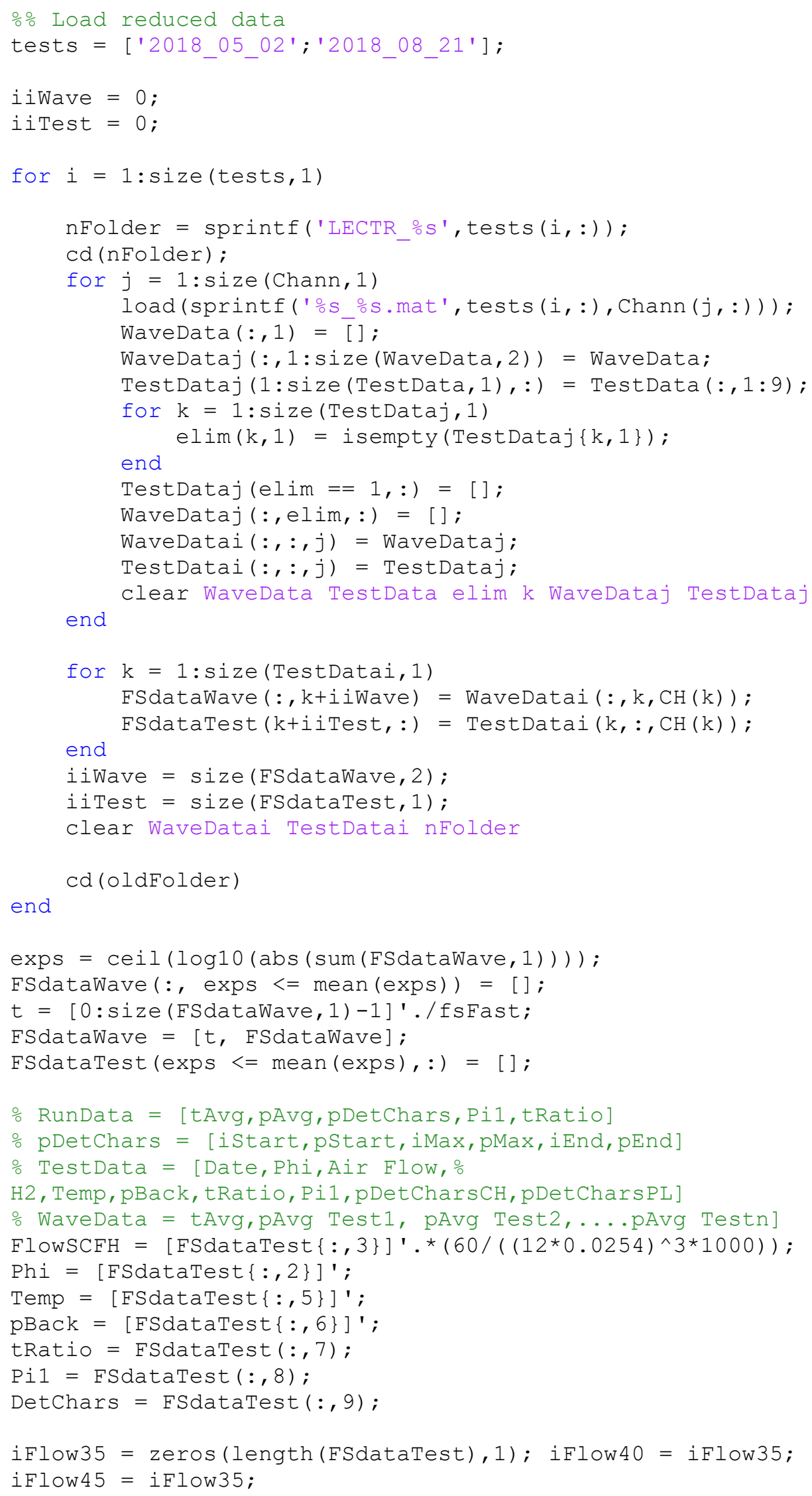




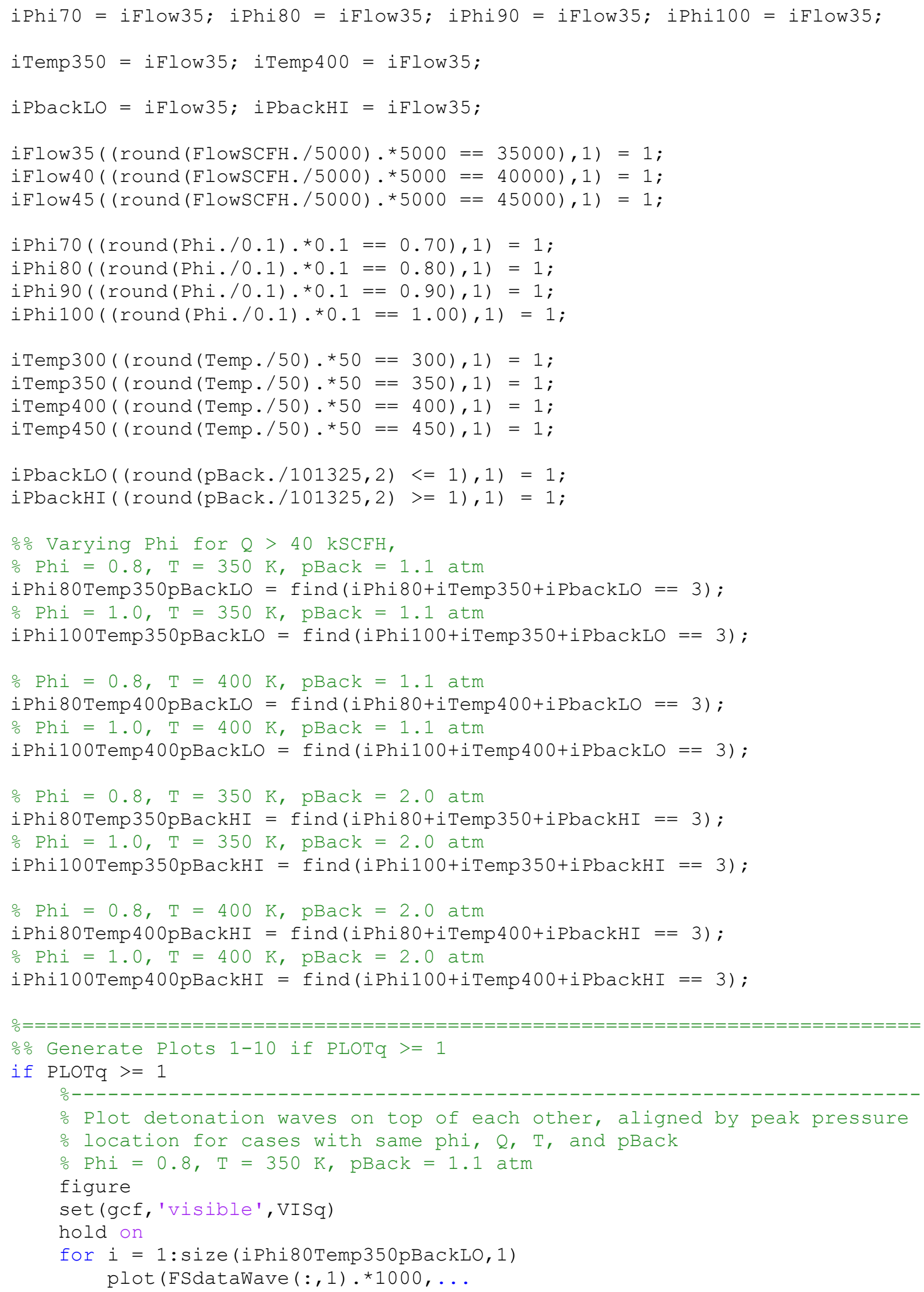


FSdataWave (: , iPhi80Temp350pBackLO (i) +1) . /1000,...

'Color',pltColor (i, :), 'Linewidth', 2)

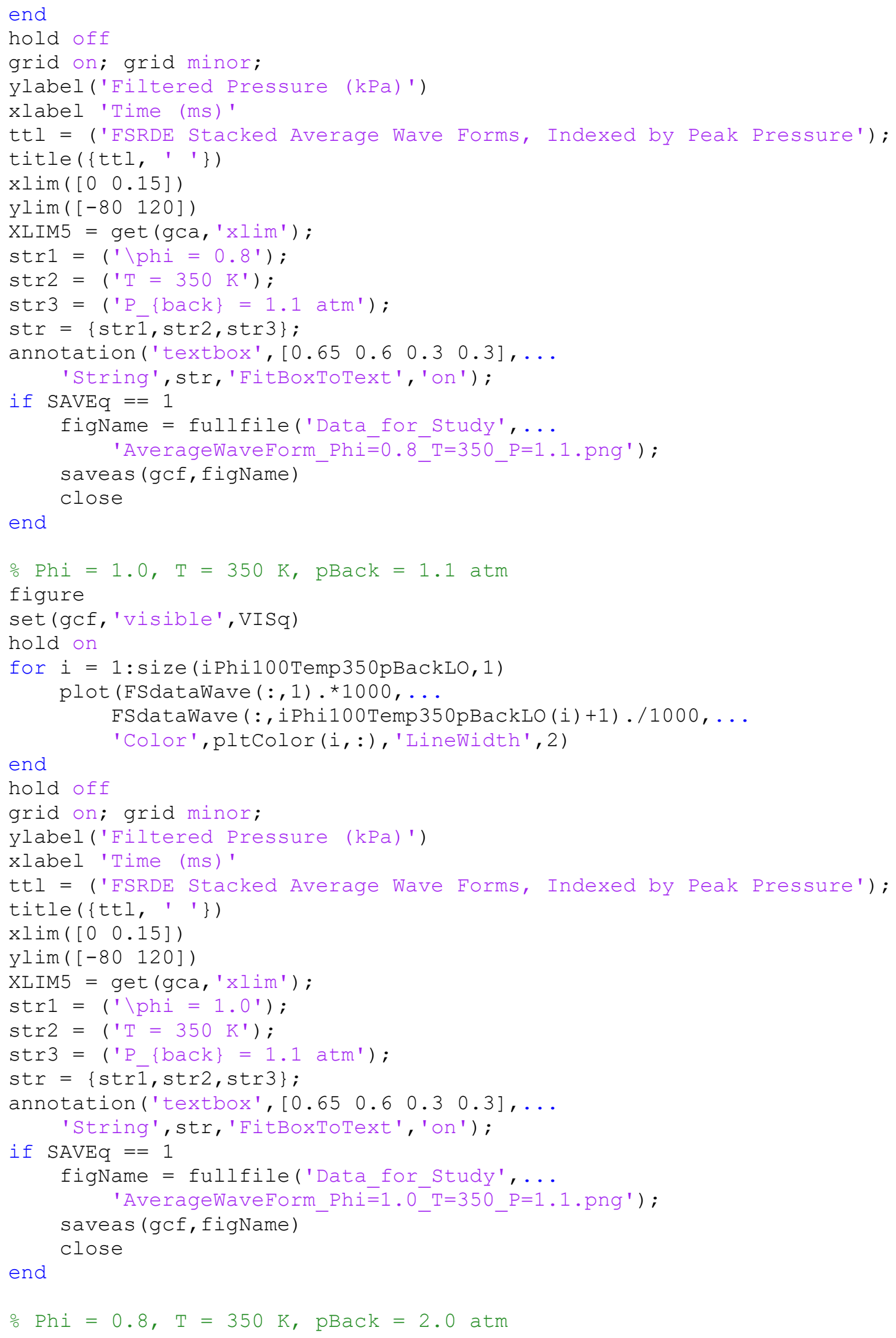




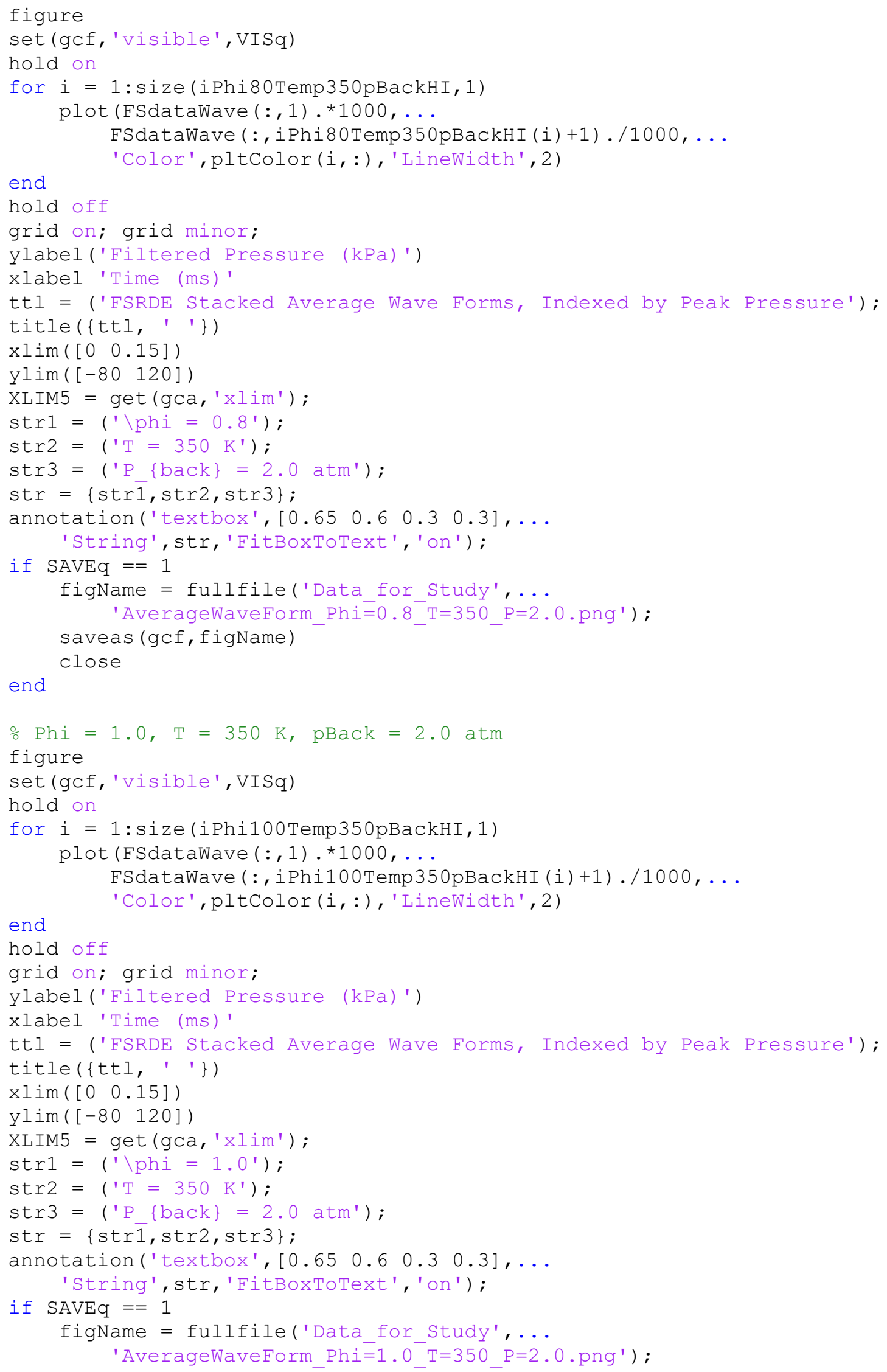




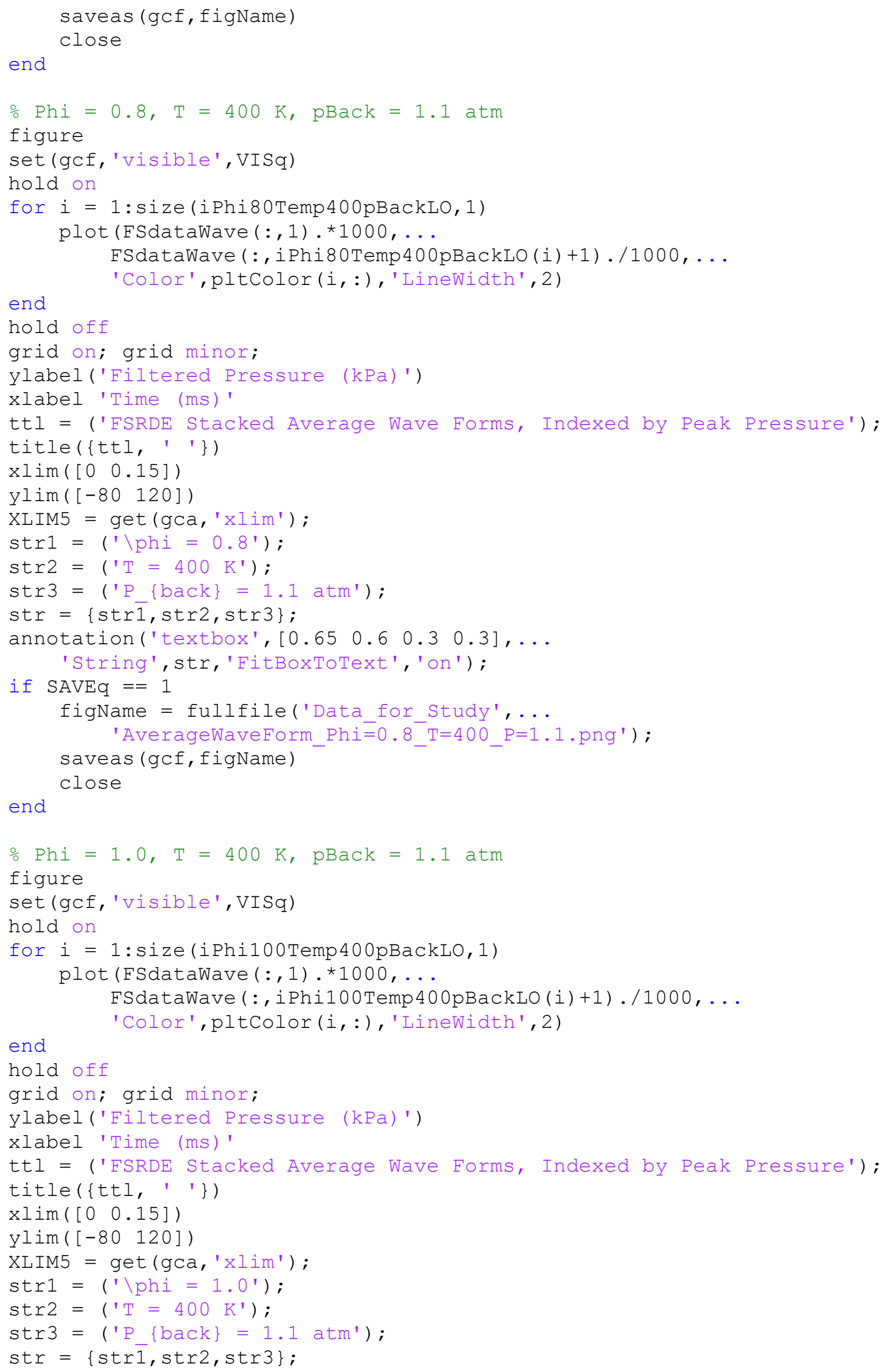




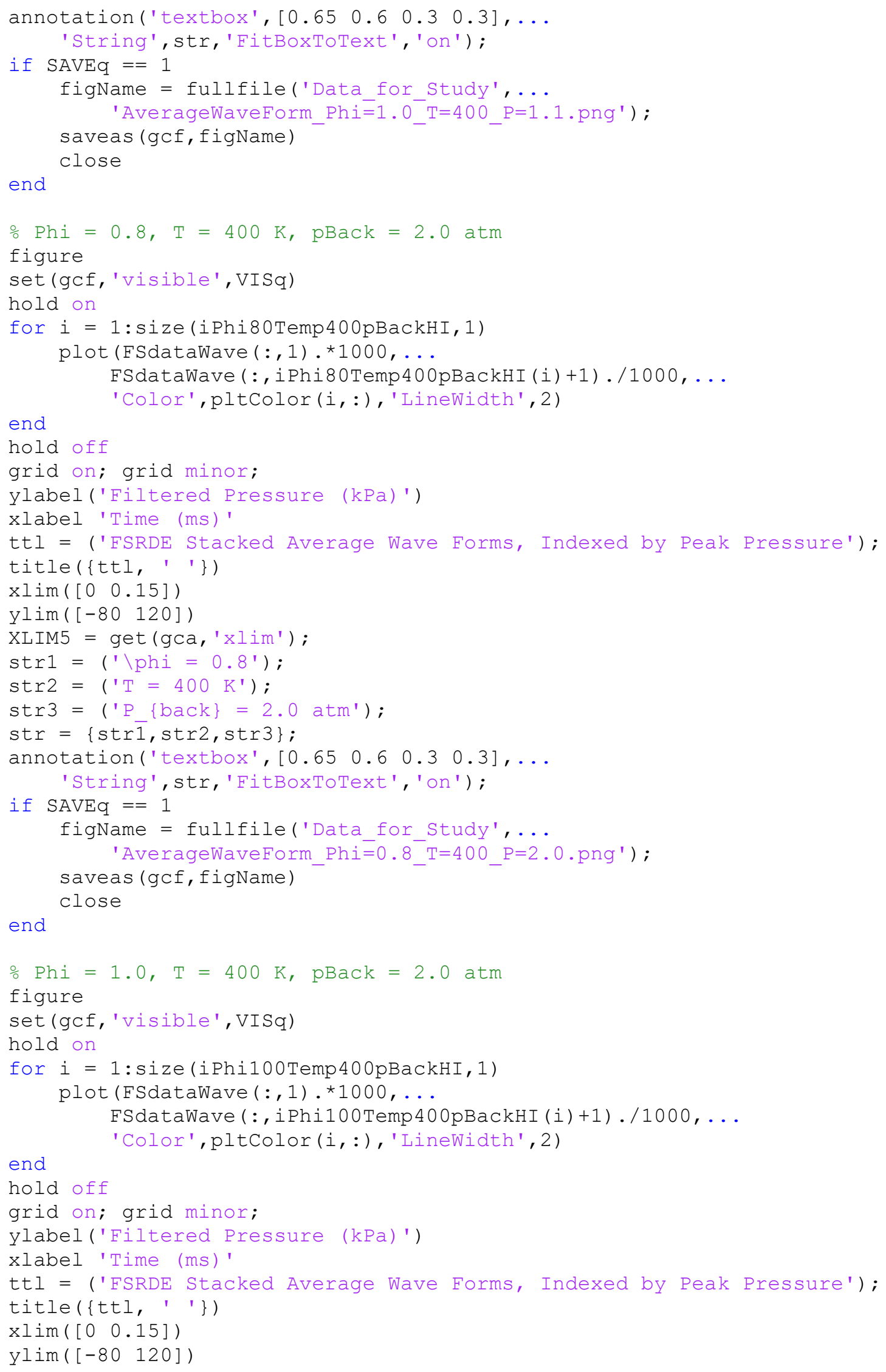




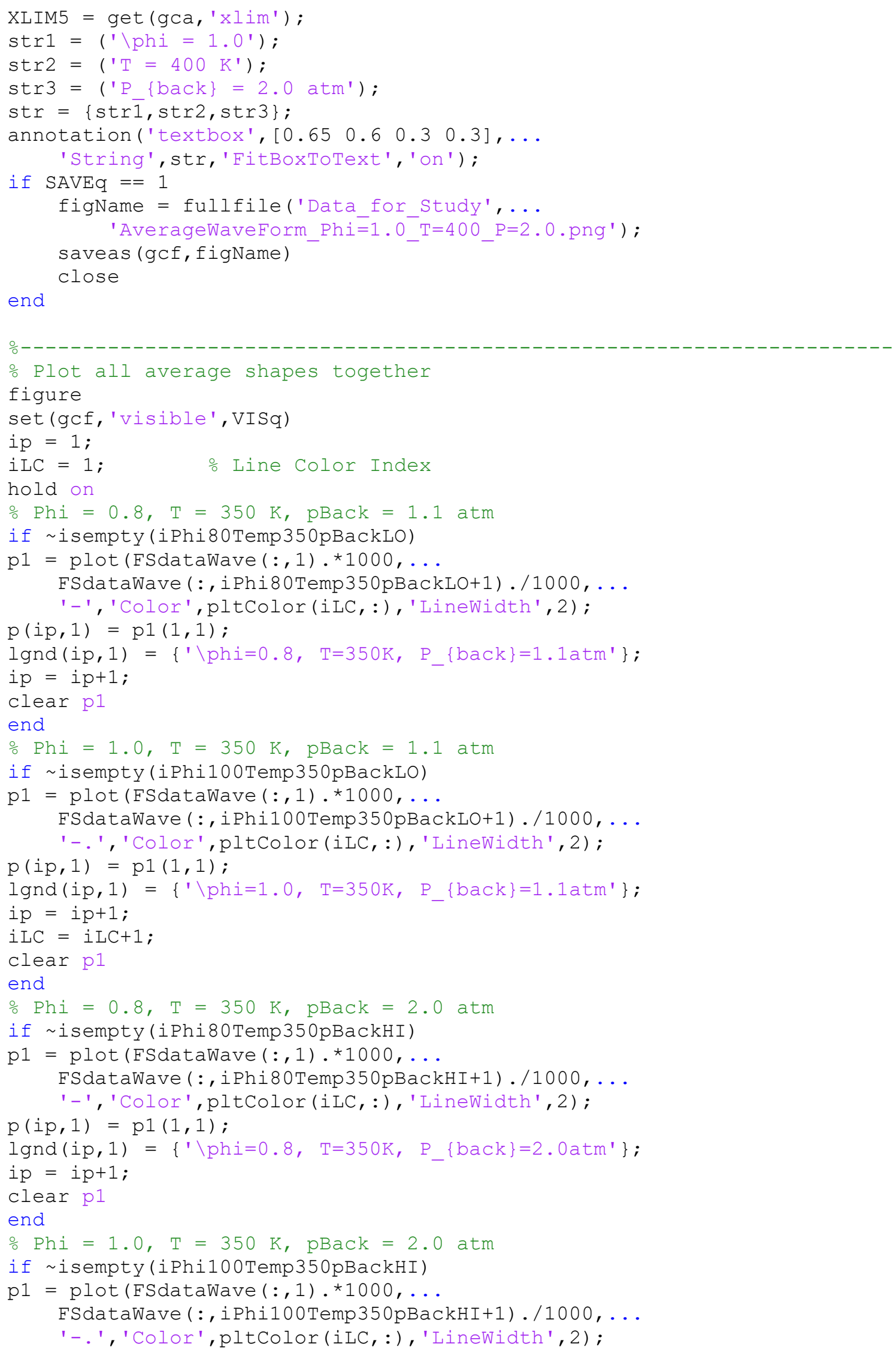




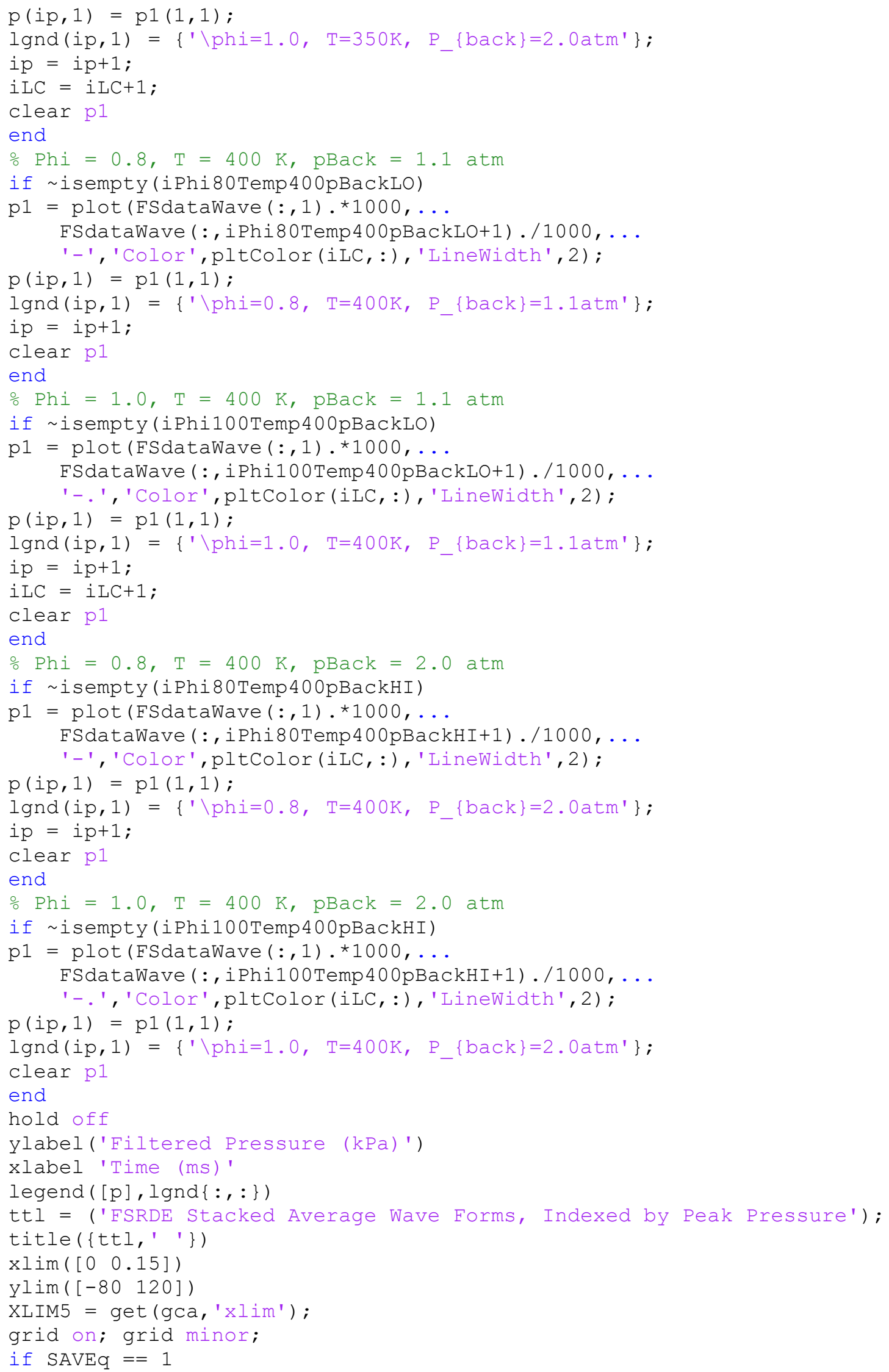




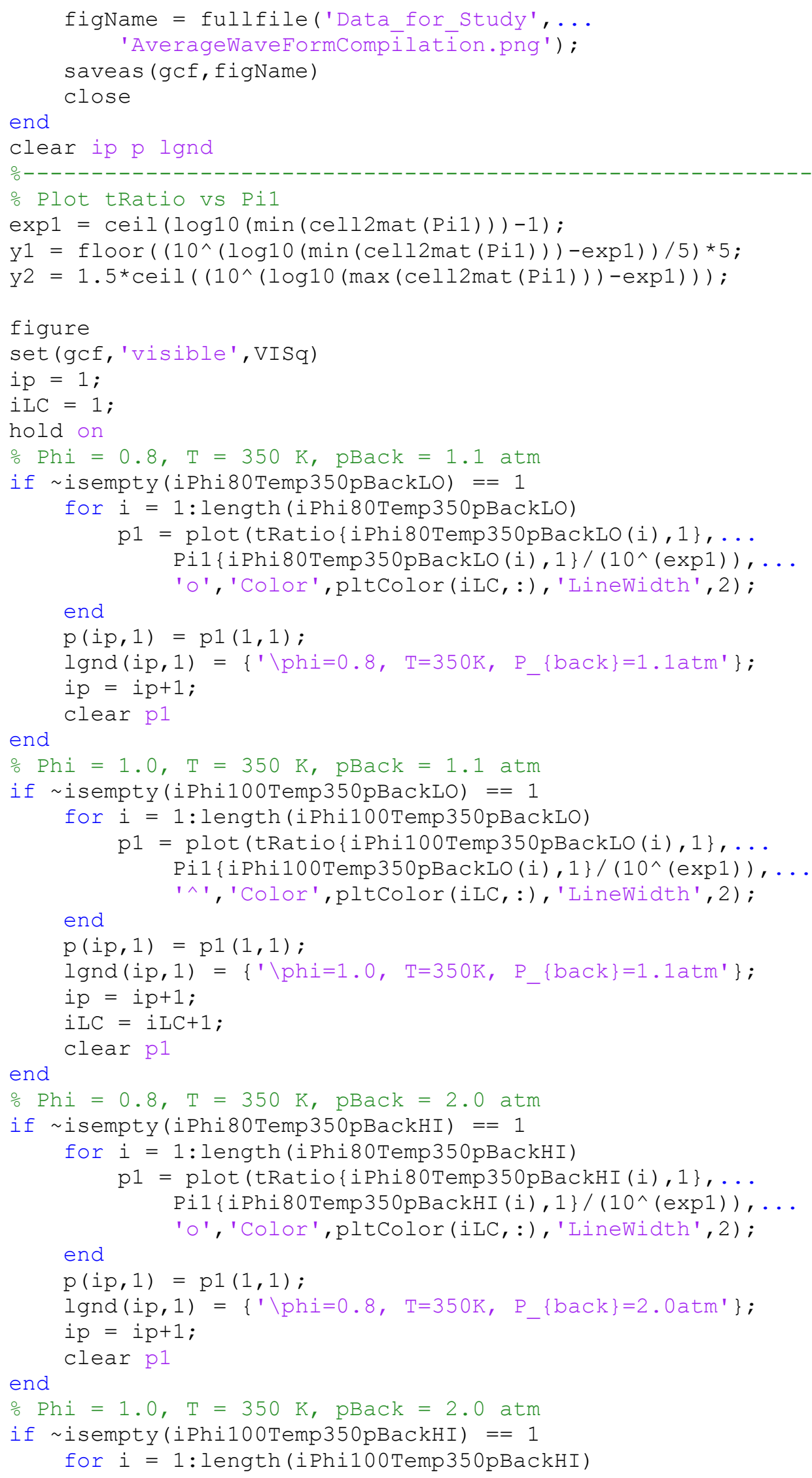




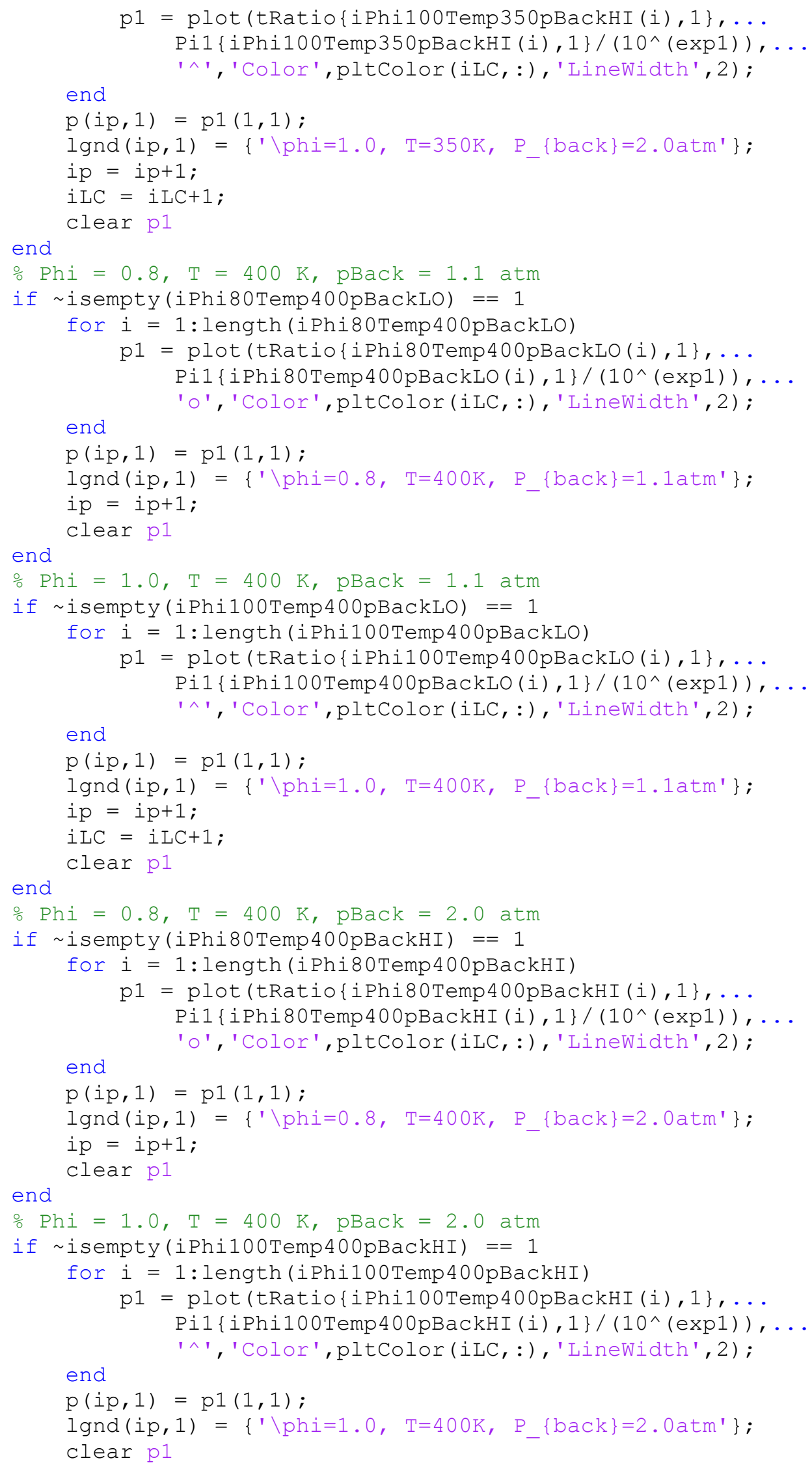




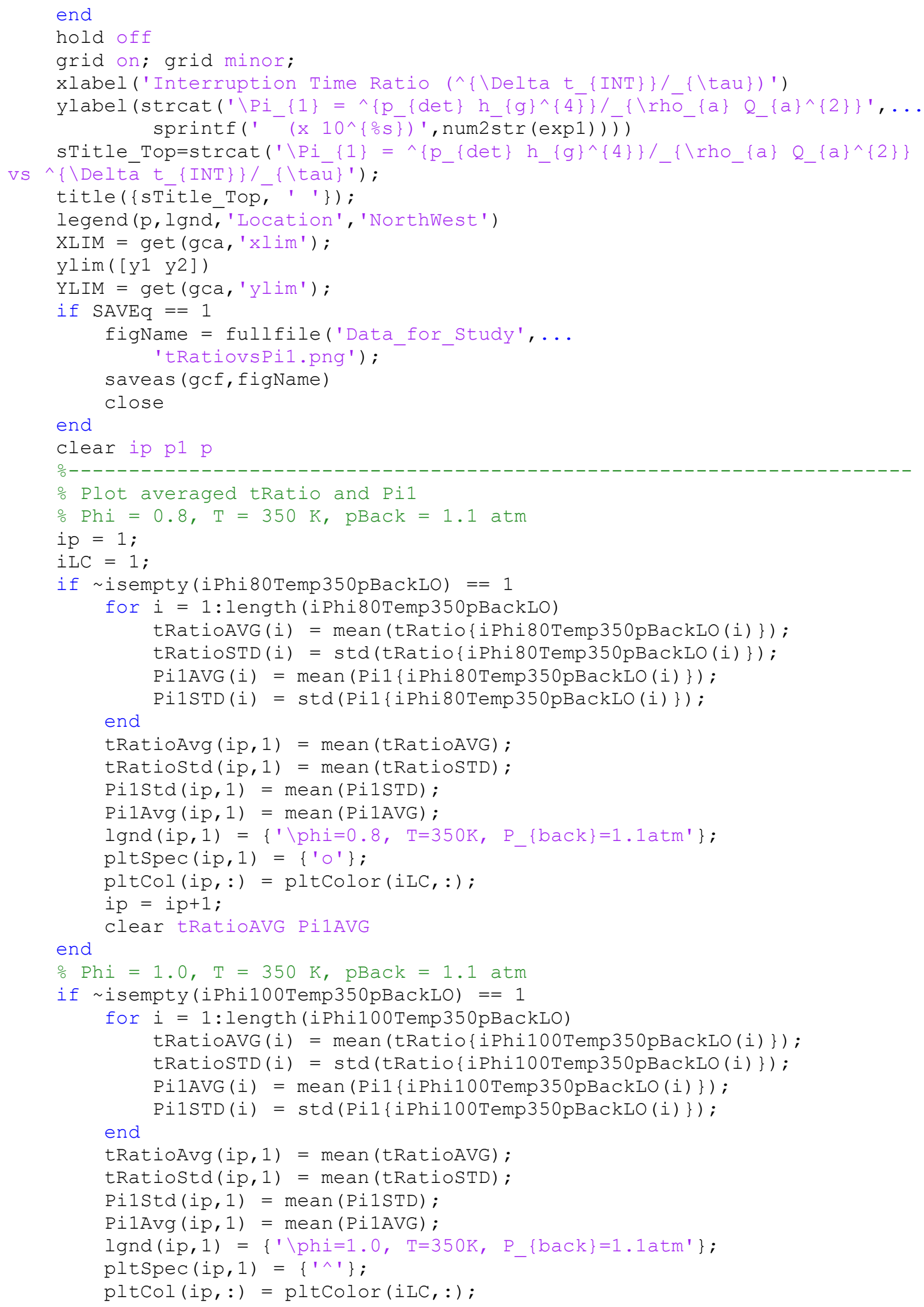




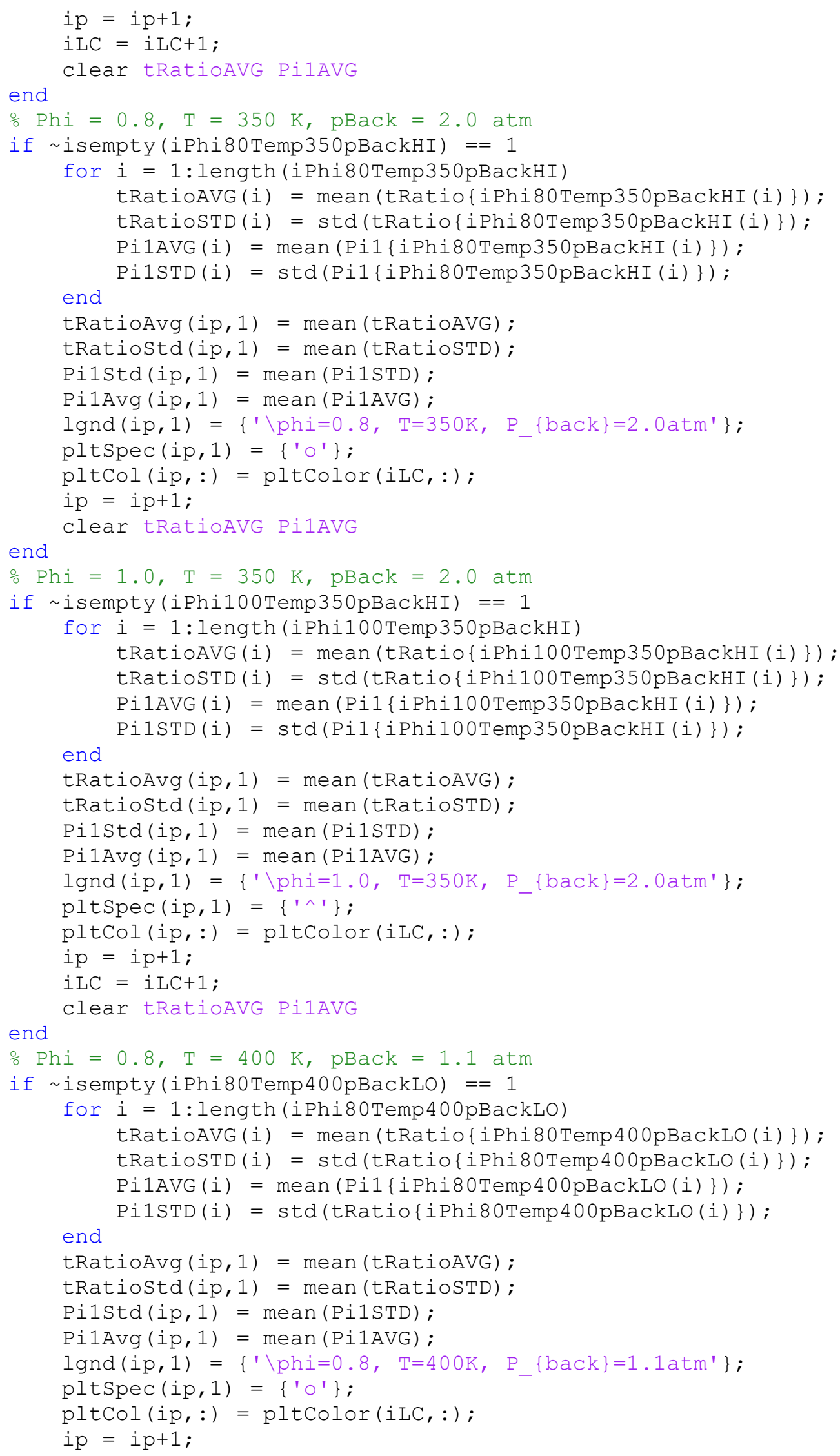




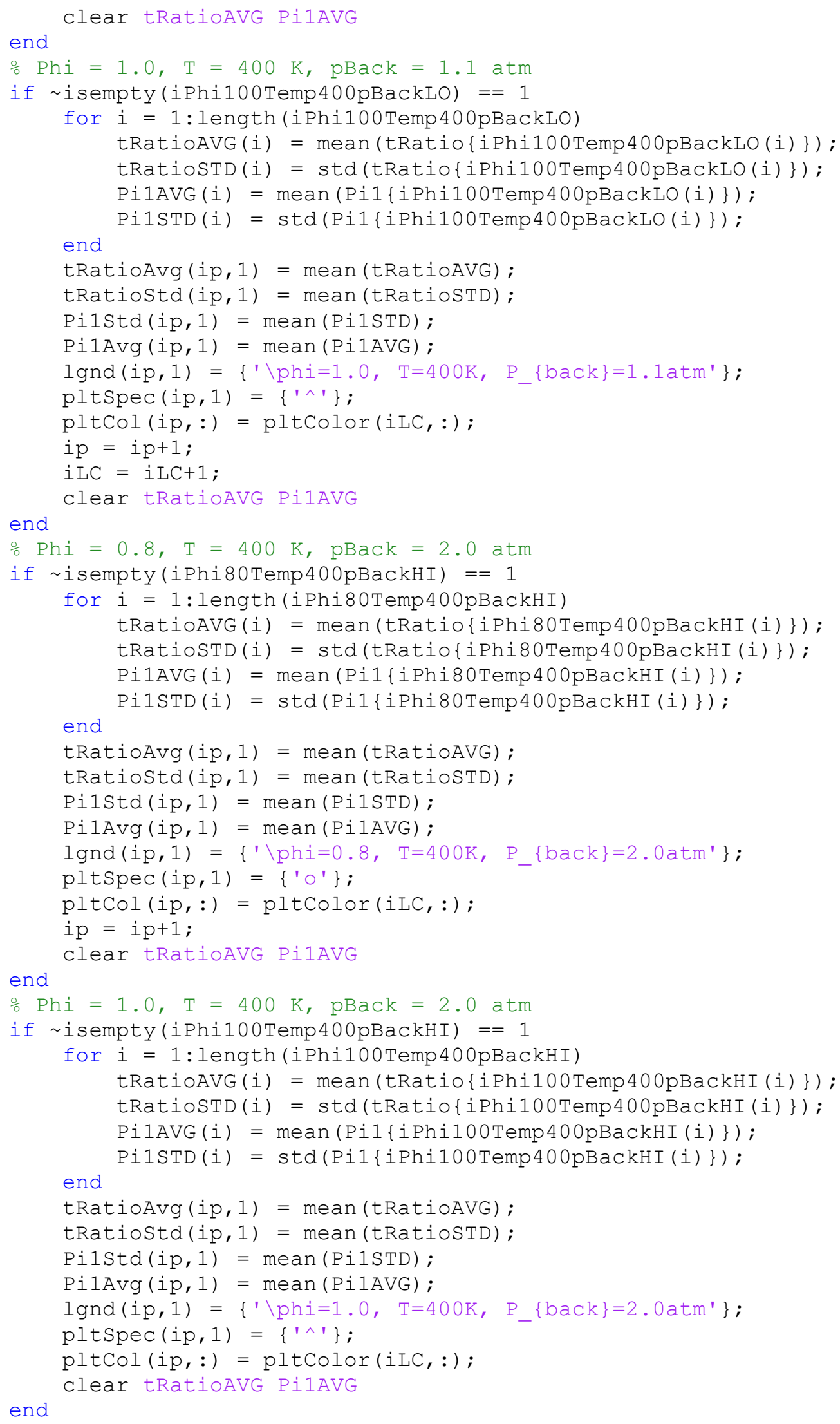




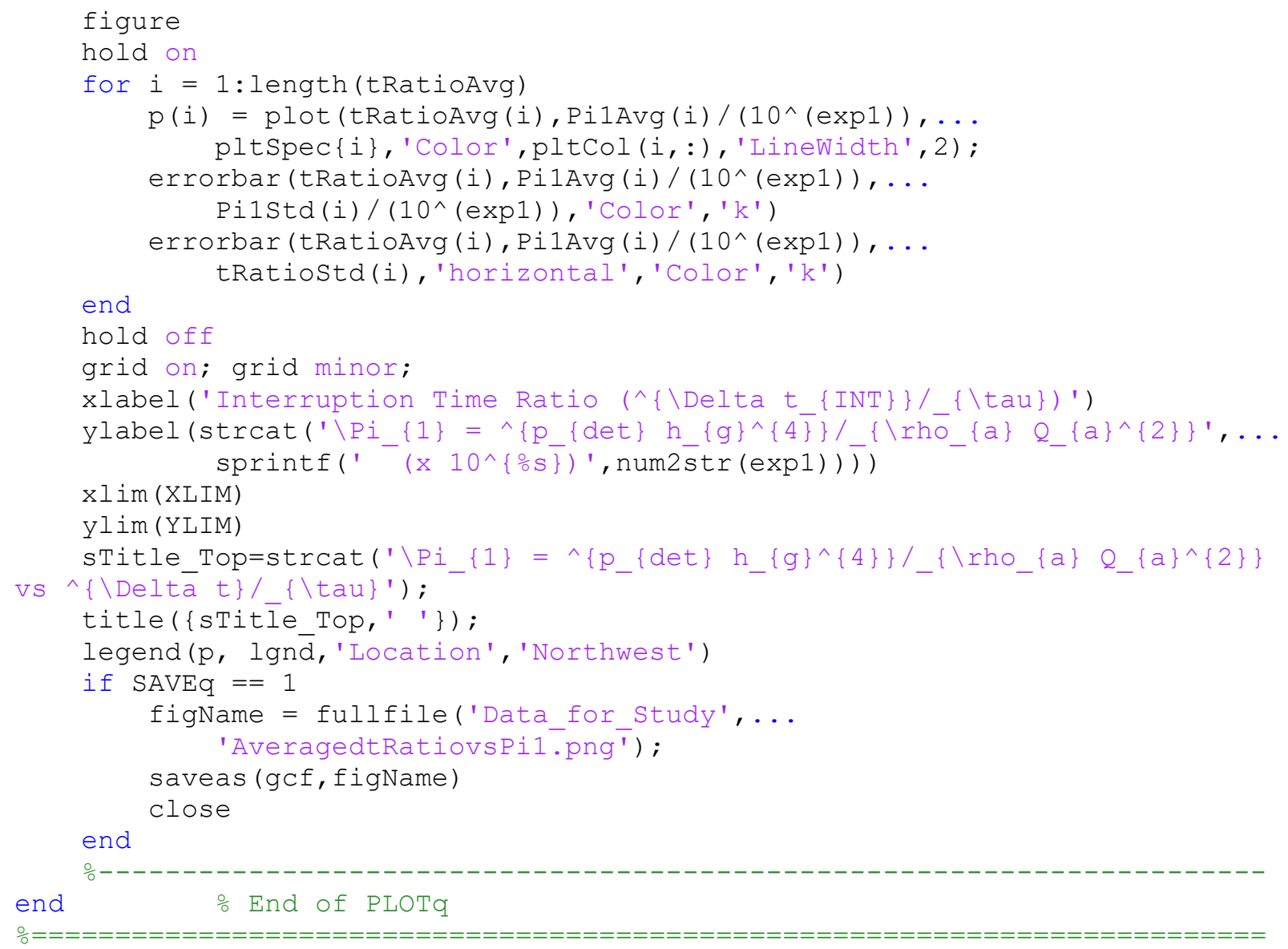




\subsection{Appendix E: FWHM MATLAB Function}

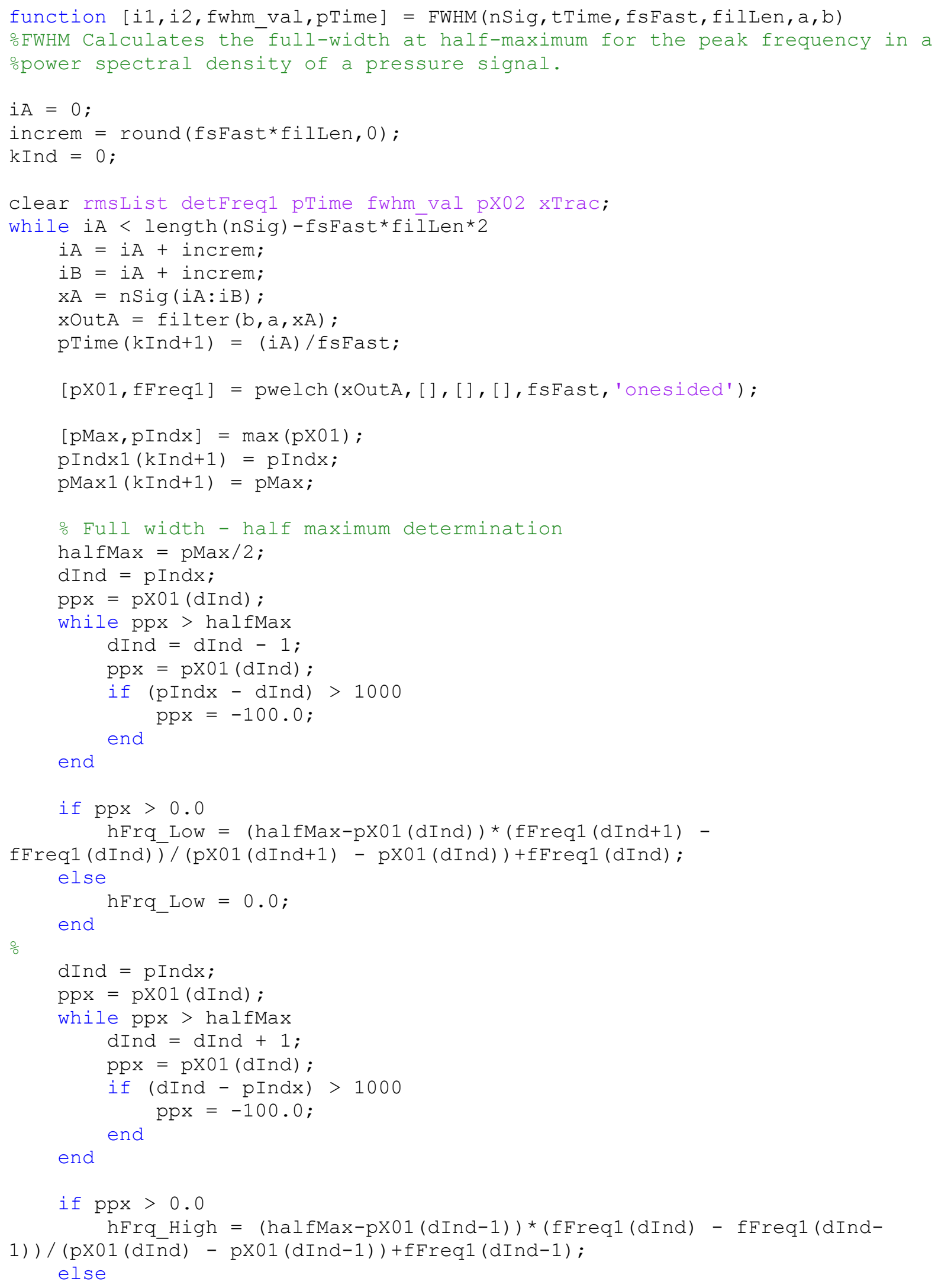




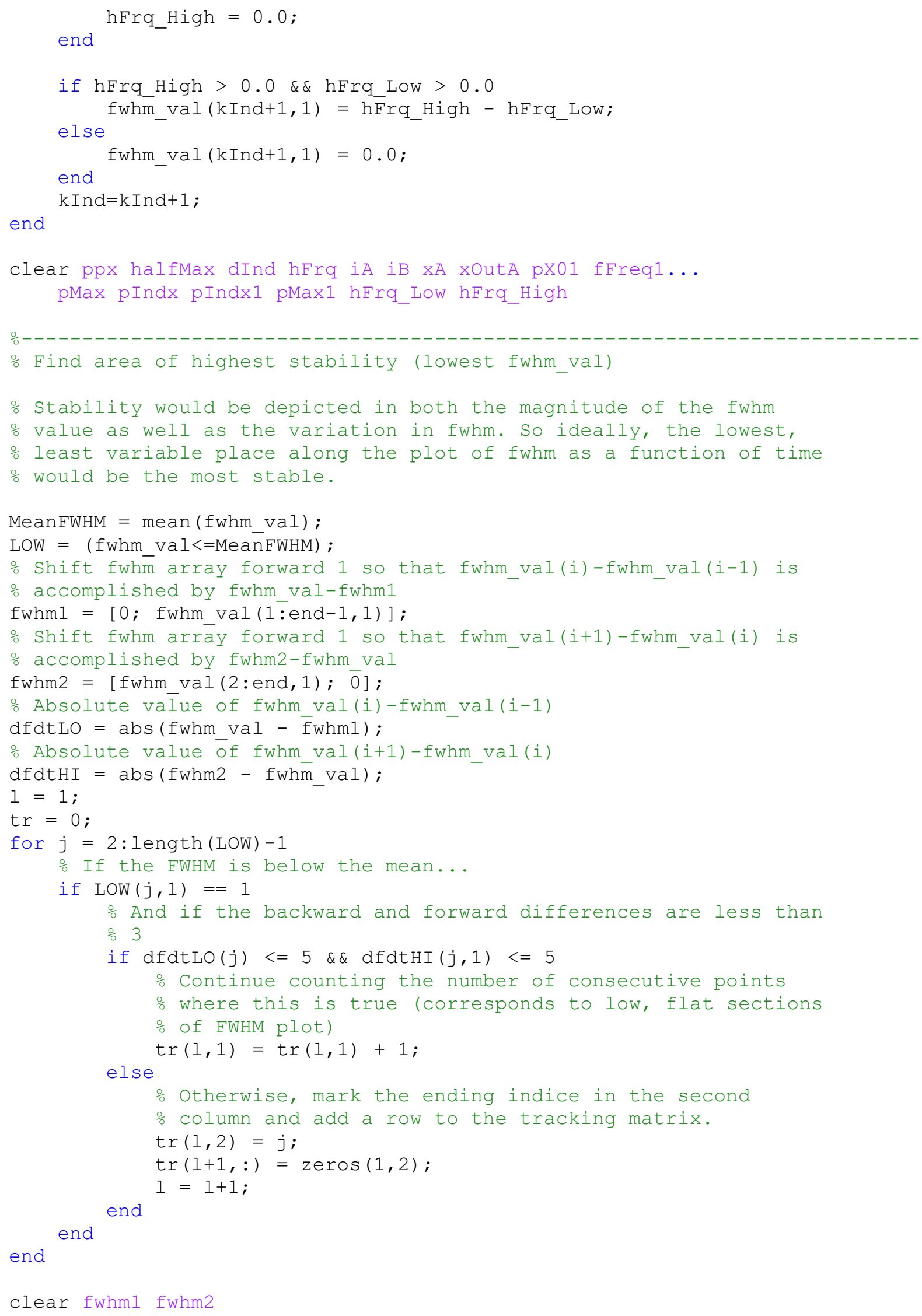


\% Find the maximum of the tracking vector within the data set.

\% If there is more than one maxima, pick the first.

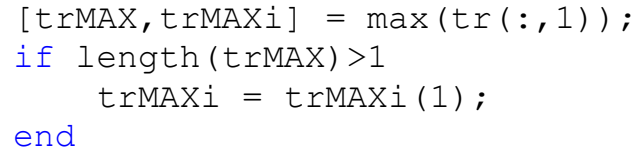




\subsection{Appendix F: Detonation Characteristics Determination MATLAB Function}

function [pDetChars] = detChars (xOut01,i1,i2, TauWave, fsFast)

opDetchars Calculate the arrival, peak, and dissipation of each wave in a opressure signal.

$\div$ Find detonation peak

\% Find locations of maximum signal

p_det $\max =\operatorname{zeros}(1,2)$;

mean ${ }^{-}=$mean $(x O u t 01(i 1: i 2,1))$;

$\mathrm{n}=1$;

for $j=i 1: i 2$

\% If the value of the signal at point $j$ is less than the mean of

\% the signal across the FWHM window, then skip it.

if $\operatorname{xOut0}(j, 1)<$ meanP

continue

end

\% Define $\mathrm{P}$ as the point before $j$ and the next 10 (total of 12

o points)

$P=\operatorname{xOut01}(j-1: j+10,1)$;

check = zeros $(12,2)$;

\% Find the maximum of $\mathrm{P}$ and the index

$[\max P, i \max P]=\max (\mathrm{P})$;

\% If the previously saved peak is greater than the current

o iteration and the difference in index for each

o "peak" is within one fifth of the period, then this is likely a

o reflected shock and should not be considered as a peak.

if P_det_max (end,2) > $\operatorname{maxP}$

if j'imaxP-2 - p_det_max (end, 1) <= TauWave*fsFast $/ 2$

continue

end

\% If the previously saved peak is less than the current iteration

\% and the difference in index for each "peak" is within one fifth

o of the period, then it could be that a preliminary peak was found

o due to noise in the signal during the initial rise. If this is

o the case, then re-assign the peak for the last wave.

elseif p_det_max (end, 2$)<\max P$

if j+imaxp-2 - p_det_max (end, 1) <= TauWave*fsFast $/ 2$

if $\mathrm{n} \sim=1$

$\mathrm{n}=\mathrm{n}-1$;

$p \_\operatorname{det} \max (n,:)=[j+i \max P-2, \quad x$ Out01 $(j+i \max P-2)]$;

$\mathrm{n}=\mathrm{n} \overline{+}$;

continue

end

end

end

o Mark which index within $\mathrm{P}$ denotes the max

check $(i \max , 2)=1$;

\% Determine where the signal is increasing for the window

o considered by $P$

for $j 2=2: 11$

Forward difference

if $P(j 2)-P(j 2-1)>0$

- The function is increasing

$\operatorname{check}(j 2,1)=1$;

end 


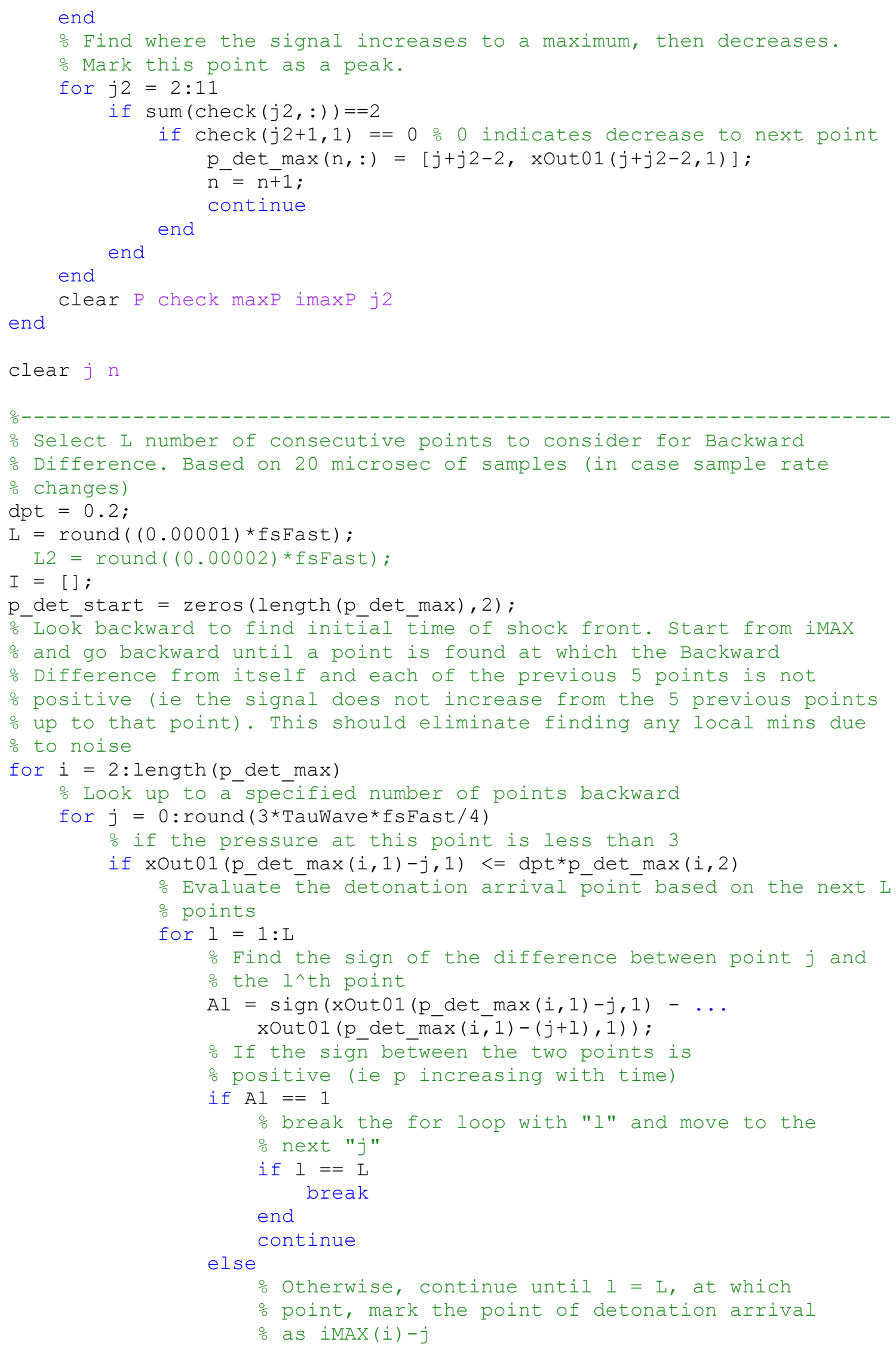




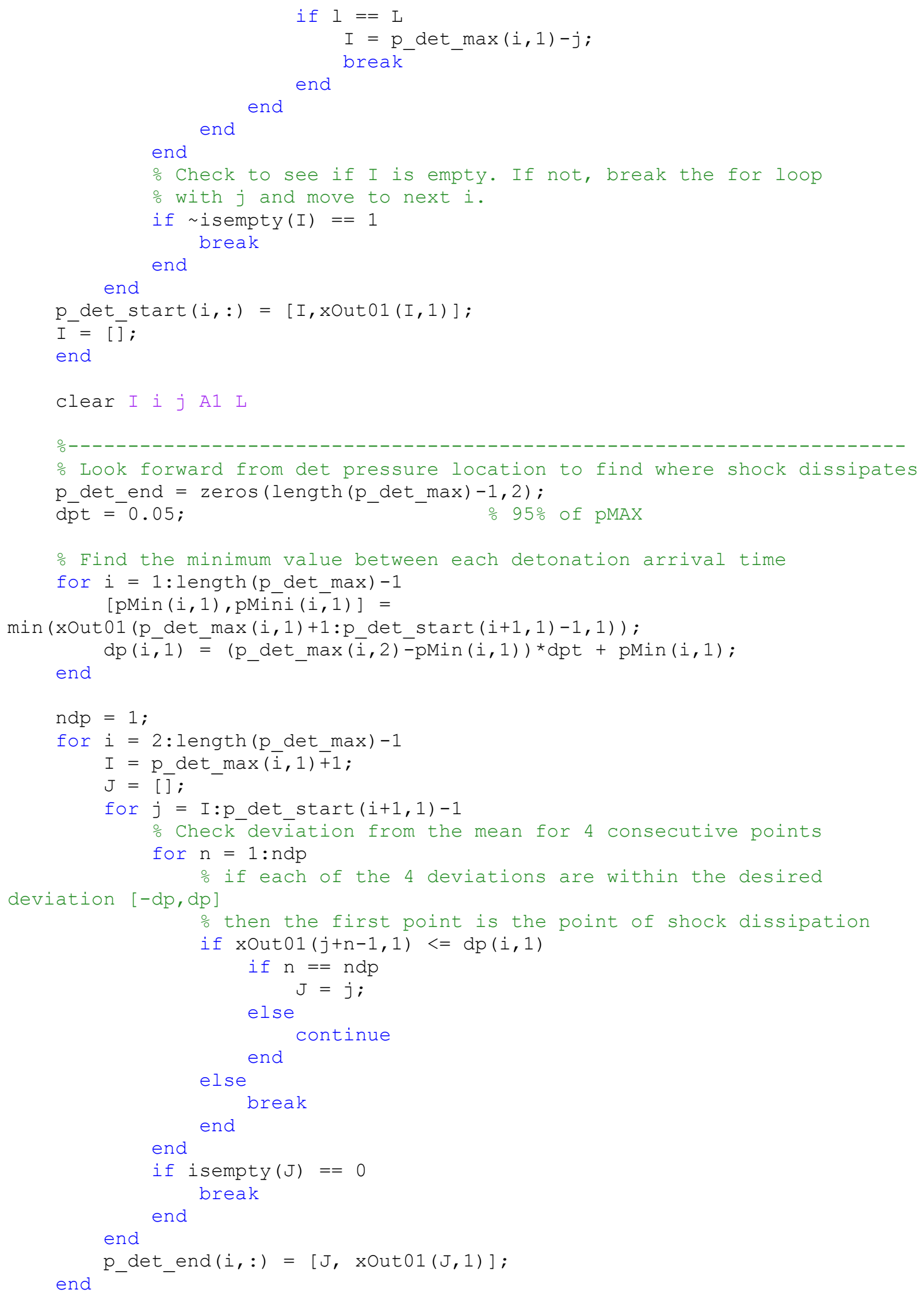




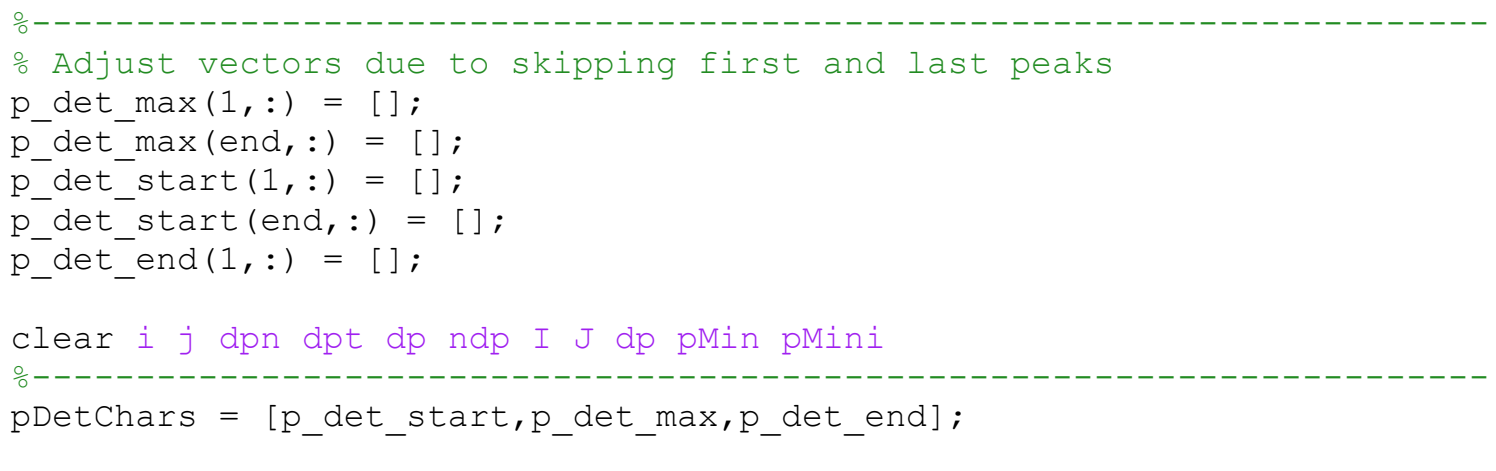

